Supporting Information

\title{
A General Organocatalytic System for Electron Donor-Acceptor Complex Photoactivation and its Use in Radical Processes
}

Eduardo de Pedro Beato, ${ }^{\dagger}$ Davide Spinnato, ${ }^{\dagger}$ Wei Zhou, ${ }^{\dagger}$ and Paolo Melchiorre*,+,

\author{
†ICIQ - Institute of Chemical Research of Catalonia, the Barcelona Institute of Science and \\ Technology, Avinguda Països Catalans 16, 43007 Tarragona, Spain \\ ${ }^{\ddagger} I C R E A$ - Passeig Lluís Companys 23, o8010 Barcelona, Spain
}

*Correspondence to: pmelchiorre@iciq.es 


\section{Table of Contents}

A. General Information $\quad$ S3

B. Substrate Synthesis $\quad$ S4

C. Experimental Procedures $\quad$ S4

C1. Experimental Setup $\quad$ S4

C2. Giese Reaction $\quad$ S8

C2.1. General Procedure A $\quad$ S8

C2.2. Characterization of Products $\quad$ S8

C2.3. General Procedure B (one-pot telescoped from acids) $\quad \mathrm{S} 10$

$\begin{array}{ll}\text { C2.4. Characterization of Products } & \text { S10 }\end{array}$

C2.5. General Procedure C (one-pot domino) S11

$\begin{array}{ll}\text { C2.6. Characterization of Products } & \text { S11 }\end{array}$

C2.7. General Procedure D (with pyridinium salts) $\quad$ S13

C2.8. Characterization of Products $\quad \mathrm{S} 13$

C2.9. General Procedure E (one-pot telescoped from amine 5) $\quad \mathrm{S} 14$

C2.10. Characterization of Products $\quad$ S14

C3. Reduction $\quad \mathrm{S} 15$

C3.1. General Procedure F (Barton decarboxylation) $\quad$ S15

$\begin{array}{ll}\text { C3.2. Characterization of Products } & \text { S15 }\end{array}$

C3.3. General Procedure G (deaminative reduction) $\quad$ S17

$\begin{array}{ll}\text { C3.4. Characterization of Products } & \text { S17 }\end{array}$

C4. $\alpha$-Alkylation of Silyl Enol Ethers $\quad$ S18

C4.1. General Procedure H $\quad$ S18

C4.2. Characterization of Products $\quad$ S18

C4.3. General Procedure I (three-component reaction) S23

$\begin{array}{ll}\text { C4.4. Characterization of Products } & \text { S23 }\end{array}$

C5. Minisci Reaction $\quad$ S26

C5.1. General Procedure J $\quad$ S26

$\begin{array}{ll}\text { C5.2. Characterization of Products } & \text { S26 }\end{array}$

C5.3. General Procedure K (enantioselective variant) S30

C5.4. Characterization of Products $\quad$ S30

$\begin{array}{ll}\text { C6. Trifluoromethylation } & \text { S31 }\end{array}$

$\begin{array}{ll}\text { C6.1. Procedure } & \text { S31 }\end{array}$

C7. Amidyl Radical Cyclization $\quad$ S32

C7.1. Procedure $\quad$ S32

C8. Large-scale Reactions $\quad$ S33

$\begin{array}{ll}\text { C8.1 Barton decarboxylation } & \text { S33 }\end{array}$

$\begin{array}{ll}\text { C8.2 Minisci reaction } & \text { S34 }\end{array}$

$\begin{array}{ll}\text { C9. Unsuccessful Substrates } & \text { S35 }\end{array}$

$\begin{array}{lr}\text { D. Mechanistic Studies } & \text { S35 }\end{array}$

D1. Control experiments

D1.1. Experiments with green light $\quad$ S35

D1.2. Optimization studies $\quad$ S35

D1.3. TEMPO trapping experiment $\quad$ S38

$\begin{array}{ll}\text { D1.4. Catalysts' stability experiments } & \text { S39 }\end{array}$

D2. UV-Vis Measurements $\quad$ S40

D3. Transient Absorption Spectroscopy $\quad$ S41

D4. CV Measurements $\quad$ S42

D5. Quantum Yield Measurements $\quad$ S46 
E. References $\quad$ S54

F. NMR Spectra $\quad$ S57

G. HPLC Traces $\quad$ S126

\section{A. General Information}

The NMR spectra were recorded at $400 \mathrm{MHz}$ and $500 \mathrm{MHz}$ for ${ }^{1} \mathrm{H}$ and 100 or $125 \mathrm{MHz}$ for ${ }^{13} \mathrm{C}$. The chemical shift $(\delta)$ for ${ }^{1} \mathrm{H}$ and ${ }^{13} \mathrm{C}$ are given in ppm relative to residual signals of the solvents $\left(\mathrm{CHCl}_{3} @ 7.26 \mathrm{ppm}{ }^{1} \mathrm{H}\right.$ NMR and $77.16 \mathrm{ppm}{ }^{13} \mathrm{C} \mathrm{NMR}$, and tetramethylsilane @ $\left.0 \mathrm{ppm}\right)$. Coupling constants are given in Hertz. The following abbreviations are used to indicate the multiplicity: s, singlet; d, doublet; q, quartet; m, multiplet; bs, broad signal; app, apparent.

High resolution mass spectra (HRMS) were obtained from the ICIQ HRMS unit on MicroTOF Focus and Maxis Impact (Bruker Daltonics) with electrospray ionization. (ESI).

UV-vis measurements were carried out on a Shimadzu UV-2401PC spectrophotometer equipped with photomultiplier detector, double beam optics and $\mathrm{D}_{2}$ and $\mathrm{W}$ light sources or an Agilent Cary60 spectrophotometer.

Emission spectra of light sources were recorded on Ocean Optics USB4000 fiber optic spectrometer.

Isolated yields refer to materials of $>95 \%$ purity as determined by ${ }^{1} \mathrm{H}$ NMR.

The authors are indebted to the team of the Research Support Area at ICIQ, particularly to the NMR and the High-Resolution Mass Spectrometry Units. Grace Fox is thanked for proofreading the manuscript.

General Procedures. All reactions were set up under an argon atmosphere in oven-dried glassware. Synthesis grade solvents were used as purchased, anhydrous solvents were taken from a commercial SPS solvent dispenser. Chromatographic purification of products was accomplished using forced-flow chromatography (FC) on silica gel (35-70 mesh). For thin layer chromatography (TLC) analysis throughout this work, Merck pre-coated TLC plates (silica gel $60 \mathrm{GF}_{254}, 0.25 \mathrm{~mm}$ ) were employed, using UV light as the visualizing agent and an acidic mixture of vanillin or basic aqueous potassium permanganate $\left(\mathrm{KMnO}_{4}\right)$ stain solutions, and heat as developing agents. Organic solutions were concentrated under reduced pressure on a Büchi rotatory evaporator.

Determination of Enantiomeric Purity. HPLC analysis on chiral stationary phase was performed on an Agilent 1200-series instrument, employing Daicel Chiralpak IC column.

Materials. Most of the starting materials used in this study are commercial and were purchased at the highest purity available from Sigma-Aldrich, Fluka, Alfa Aesar, Fluorochem, and used as received, without further purifications. 


\section{B: Substrate Syntheses}

The following substrates were synthesized according to reported procedures (Figure S1). ${ }^{1-14}$

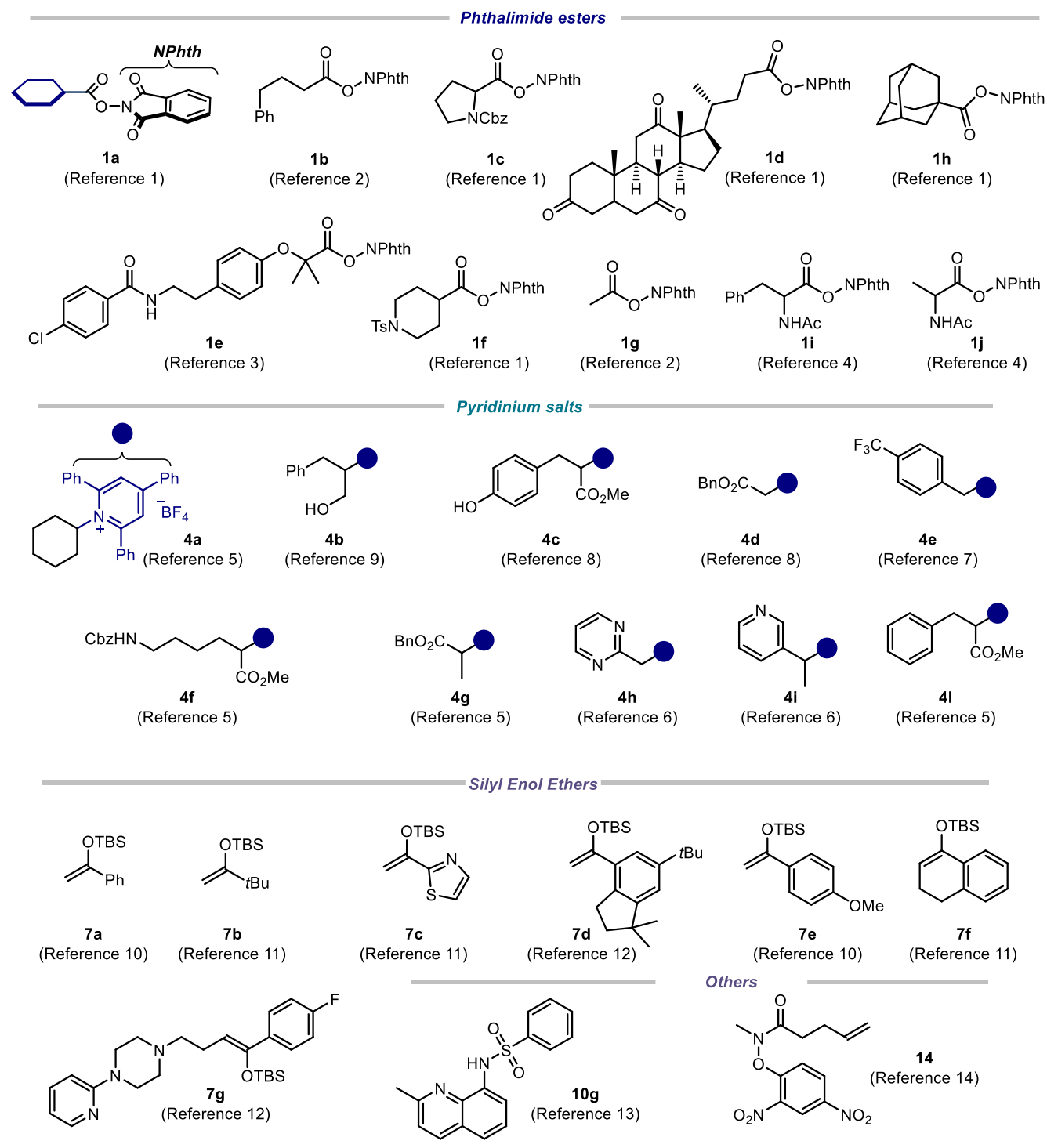

Figure S1: Starting materials synthesized according to known procedures.

\section{Experimental Procedures}

\section{C1. Experimental Setup}

\section{- Set-up $13 D$ printed reactor with LED strip}

For reactions performed using a blue LED strip as the light source, a 3D-printed photoreactor was used, consisting of a $9 \mathrm{~cm}$ diameter crystallizing dish with a 3D printed support of 6 positions, and a hole of $22 \mathrm{~mm}$ in the middle to allow ventilation (Figure S2, left). A commercial 1-meter LED strip was wrapped around the crystallizing dish, while a fan was used to cool down the reactor (the reaction temperature was measured to be between $35-40^{\circ} \mathrm{C}$ ). Each of the positions could be used to fit a standard $16 \mathrm{~mm}$ diameter vial with a Teflon screw cap. Experiments at 465 nm were conducted using a 1m strip, 14.4W "LEDXON MODULAR 9009083 LED, SINGLE 
5050" purchased from Farnell, catalog number 9009083. The emission spectrum of these LEDs is shown in Figure S2, right panel.
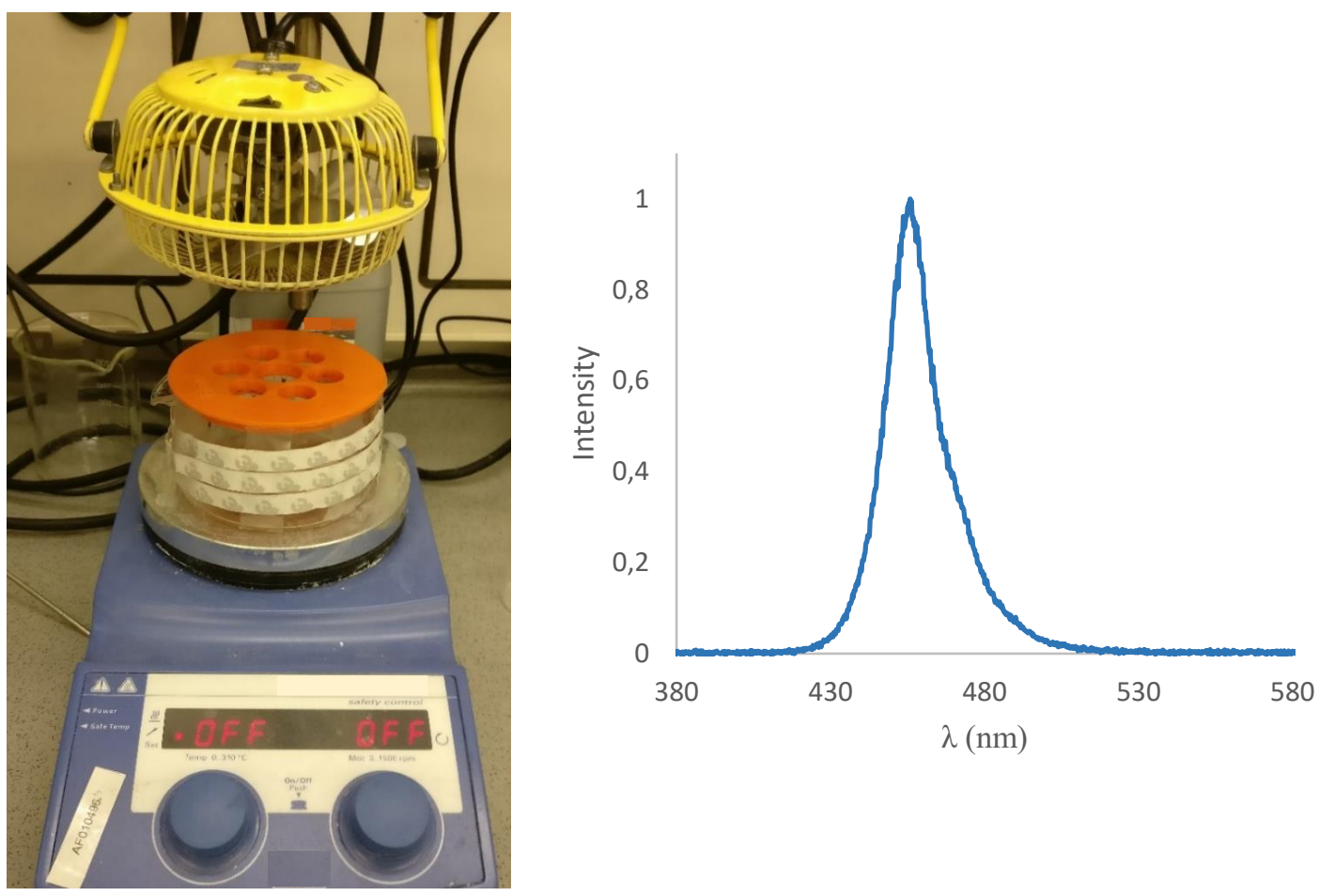

Figure S2: Blue LEDs photoreactor used for reactions where temperature control was not needed (left). Emission spectrum of the $465 \mathrm{~nm}$ LED strip used in this reactor (right).

\section{- $\quad$ Set-up 2 Kessil Lamp setup}

For reactions performed with a Kessil lamp, the irradiation set-up consisted of a $50 \mathrm{~W}$ Kessil blue LED lamp (PR160L-456, 100\% intensity, 2-3 cm away - Figure S3).

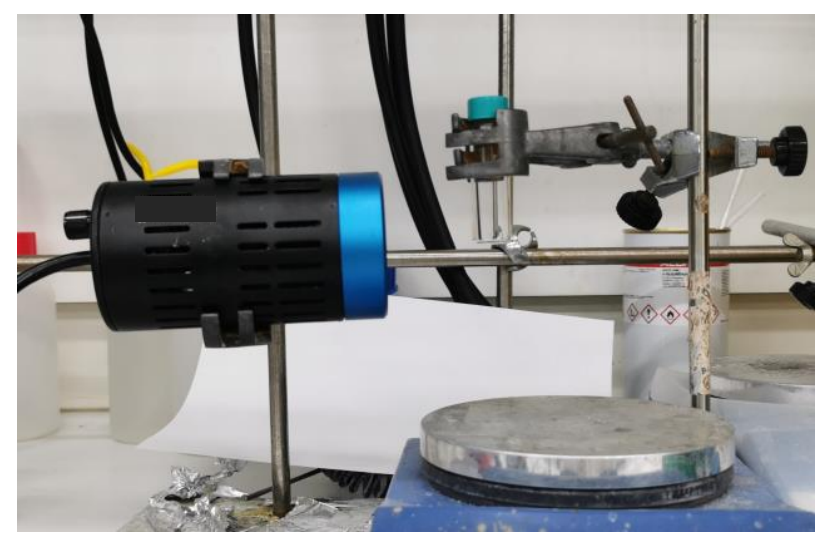

Figure S3: Kessil lamp set-up.

- Set-up 3 Temperature-controlled 4-position reactor with LED strip

For reactions where temperature control was employed, the photoreactor consisted of a $12.5 \mathrm{~cm}$ diameter jar fitted with 4 standard B29 size quickfit-glass joints arranged around a central B29size 
joint. A commercial 1-meter LED strip was wrapped around the jar, followed by a layer of aluminium foil and cotton for insulation (Figure S4).

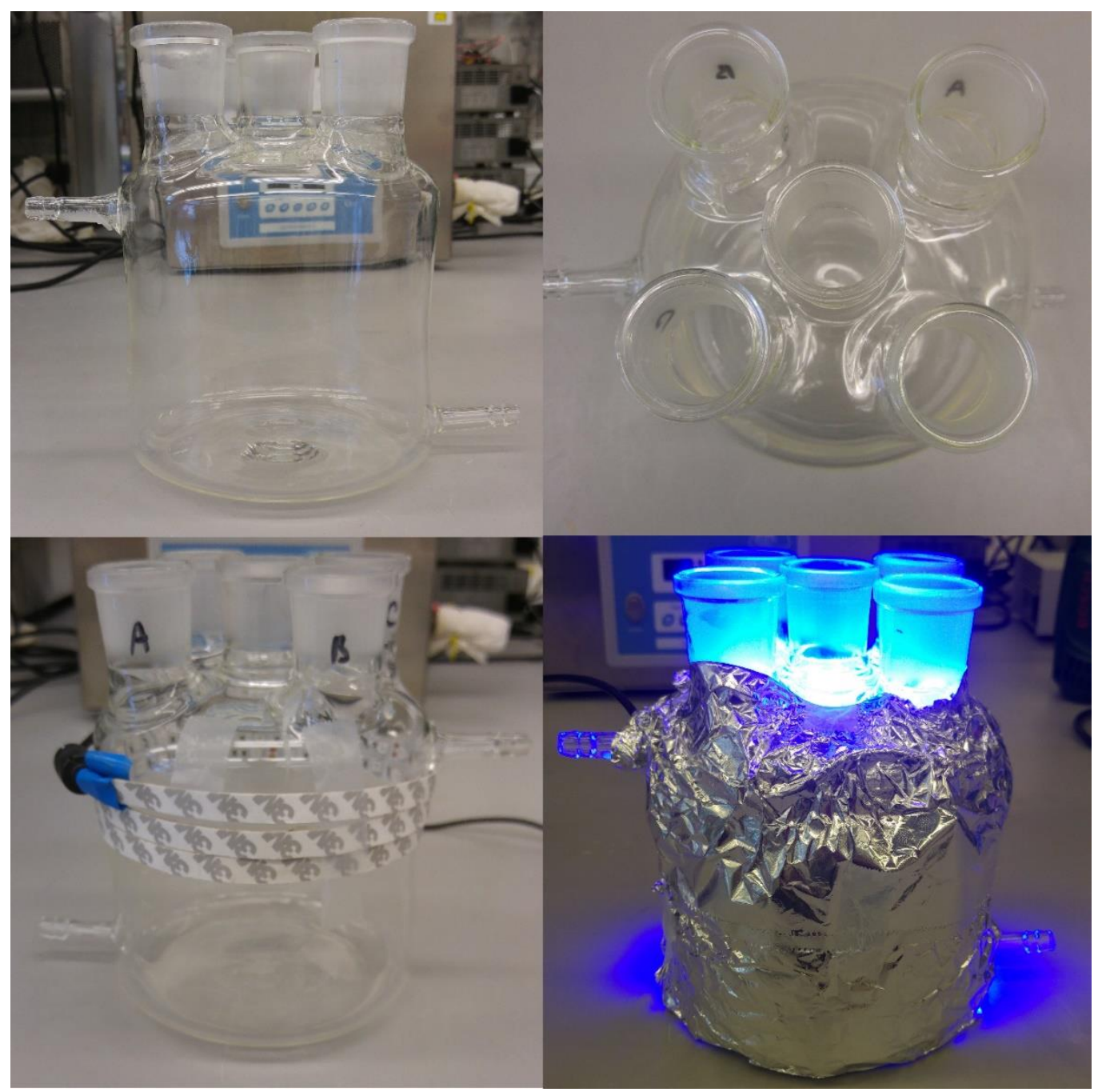

Figure S4: Photoreactor used for temperature-controlled reactions - pictures taken at different stages of the set-up assembly.

Each of the joints could be used to fit a standard $16 \mathrm{~mm}$ or $25 \mathrm{~mm}$ diameter Schlenk tube with a Teflon adaptor (Figure S5).

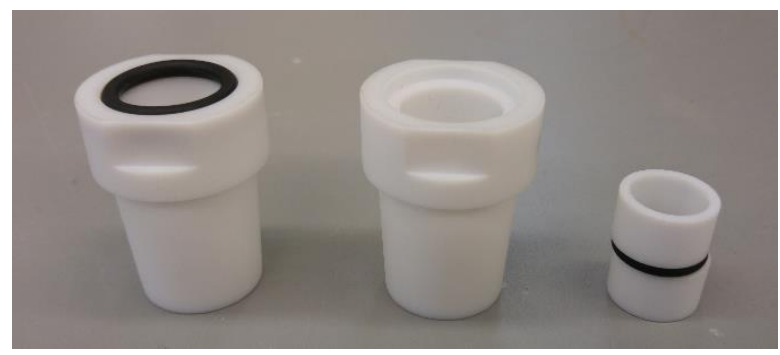

Figure S5: Teflon adaptors to use Schlenk tubes in the photoreactor.

An inlet/outlet system provided circulation of liquid (ethylene glycol/water mixture) from a Huber Minichiller 300 inside the jar. This setup allowed the performance of reactions at temperatures ranging from $-20^{\circ} \mathrm{C}$ to $80^{\circ} \mathrm{C}$ with accurate control of the reaction temperature $\left( \pm 1^{\circ} \mathrm{C}\right.$, Figure S6). 


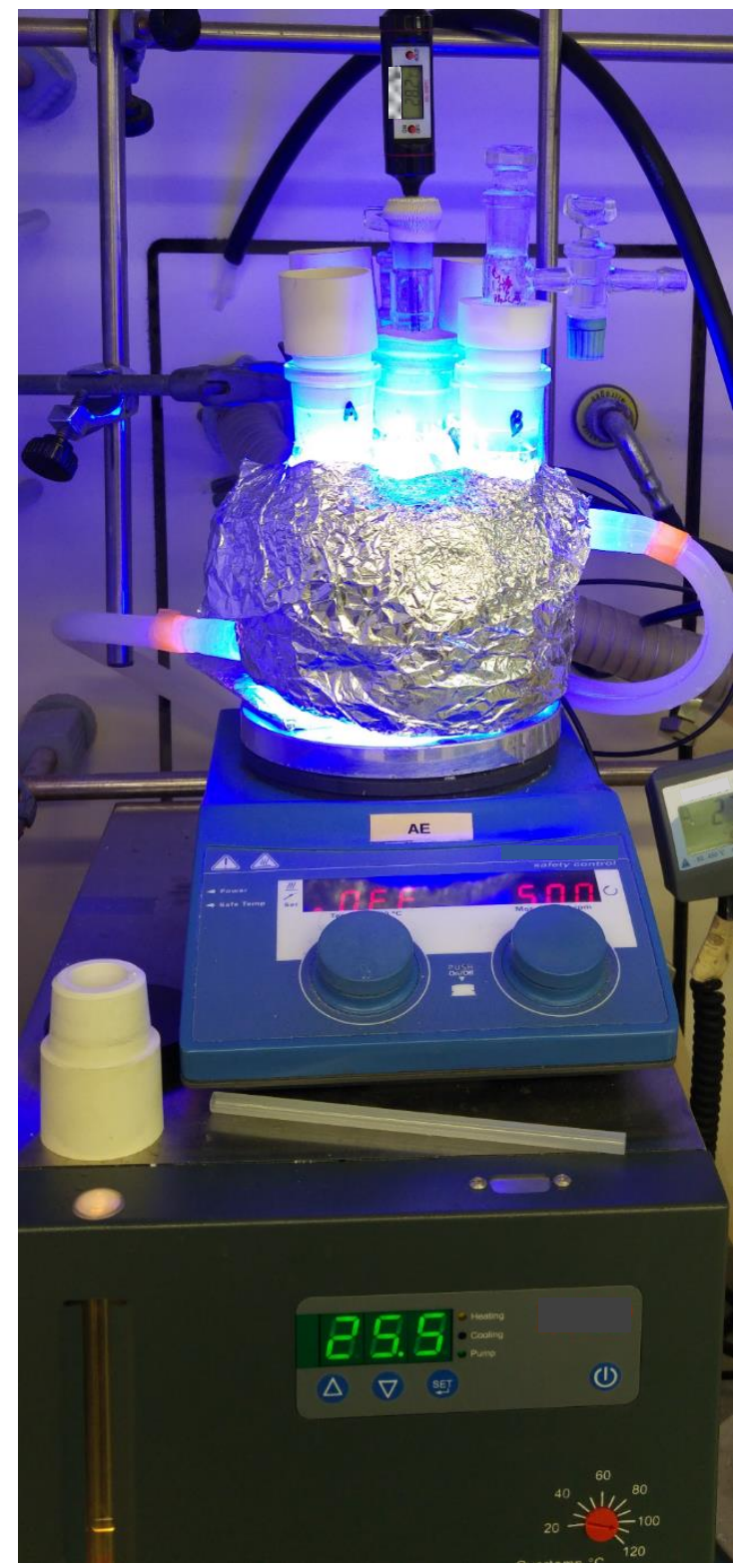

Figure S6: Fully assembled temperature-controlled photoreactor in operation.

In order to maintain consistent illumination between different experiments, only the four external positions were used to perform reactions. The central position was used to monitor the temperature using a thermometer inside another inserted Schlenk tube identical to those used to perform reactions, ensuring that the reaction mixtures were at the desired temperature.

\section{Set-up 4 Temperature controlled one-position reactor with LED strip}

Our photoreactor for the enantioselective version of the Minisci reaction consisted of a consisted of a $4 \mathrm{~cm}$ diameter jar fitted with a standard 29 sized ground glass joint. A commercial 1 meter LED strip was wrapped around the jar, followed by a layer of aluminium foil and cotton for insulation. An inlet and an outlet allow the circulation of liquid from a Huber Minichiller 300 inside the jar. This setup allows to perform reactions at temperatures ranging from $-20^{\circ} \mathrm{C}$ to $80{ }^{\circ} \mathrm{C}$ with accurate control of the reaction temperature $\left( \pm 1{ }^{\circ} \mathrm{C}\right)$. An inlet and an outlet allow the circulation of liquid from a Huber Minichiller 300 inside the jar. This setup allows to perform reactions at temperatures ranging from $-20^{\circ} \mathrm{C}$ to $80{ }^{\circ} \mathrm{C}$ with accurate control of the reaction temperature $\left( \pm 1^{\circ} \mathrm{C}\right.$, Figure S5). 


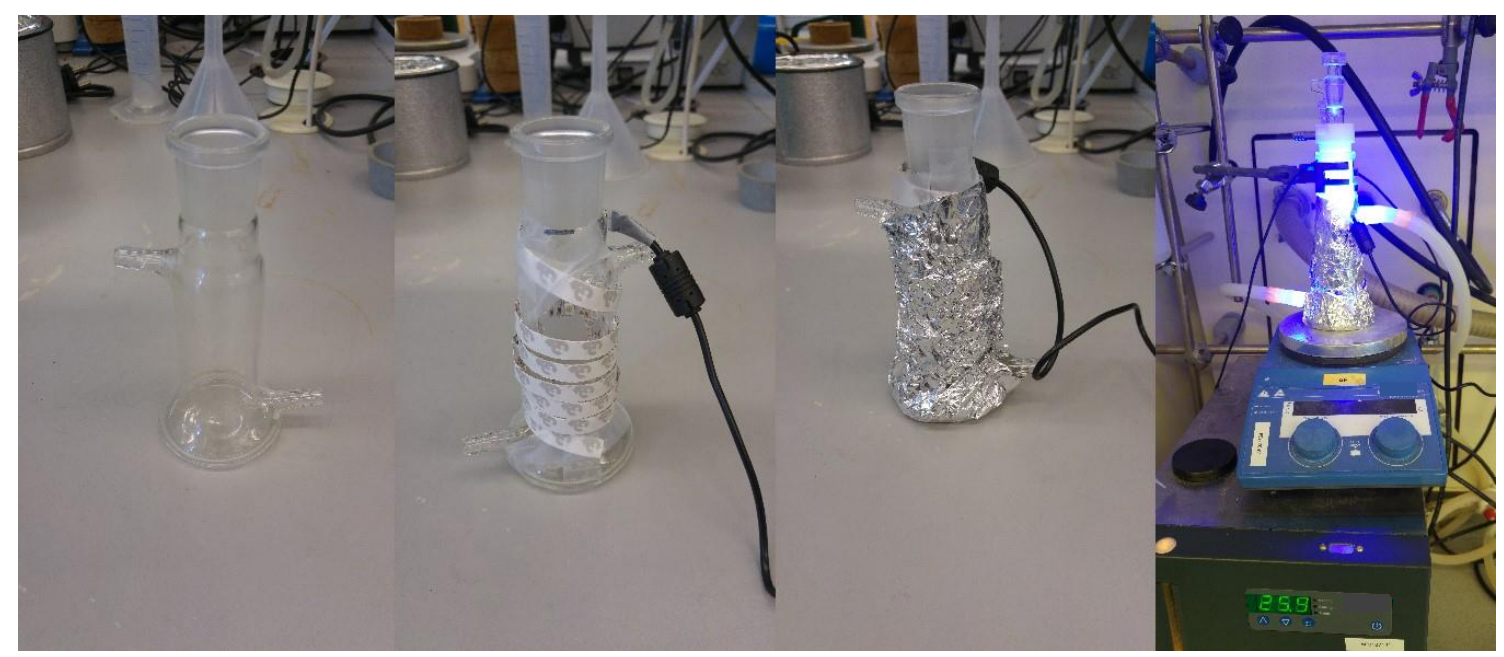

Figure S7: Fully assembled controlled temperature photoreactor in operation for enantioselective Minisci reaction.

\section{C2. Giese Addition}

\section{C2.1 General Procedure A}
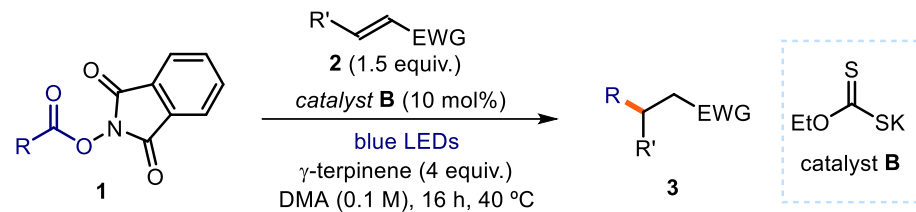

Reactions performed using set-up 1 in Figure S2. In an oven dried vial with a Teflon septum screw cap, potassium ethyl xanthogenate B $(3.2 \mathrm{mg}, 0.02 \mathrm{mmol}, 0.1$ equiv.), $\mathrm{N}$ hydroxyphthalimide ester 1 ( $0.2 \mathrm{mmol}, 1$ equiv.) and the electron-poor olefin 2 ( $0.3 \mathrm{mmol}, 1.5$ equiv., if solid), were dissolved in DMA ( $2 \mathrm{~mL}$, synthesis grade solvent). Then, $\gamma$-terpinene (128 $\mu \mathrm{L}, 0.8 \mathrm{mmol}, 4$ equiv.) was added. The resulting orange mixture was degassed with argon sparging for 60 seconds. If the electron-poor olefin 2 was liquid, it was added via syringe after the argon sparging. The vial was then placed in the 3D printed support photoreactor (Figure S2) and irradiated under stirring for 16 hours, unless otherwise specified. The mixture was transferred to an extraction funnel, $\mathrm{NaOH} 1 \mathrm{M}$ solution was added, and the organic layer was extracted with DCM. The organic layer was washed with brine twice. The combined organic layers were dried over anhydrous $\mathrm{MgSO}_{4}$, filtered, and concentrated to dryness. The crude residue was purified by column chromatography to afford the corresponding product $\mathbf{3}$ in the stated yield with $>95 \%$ purity according to ${ }^{1} \mathrm{H}$ NMR analysis.

\section{C2.2 Characterization of Products with General Procedure A}

2-cyclohexylethyl)sulfonyl)benzene (3a): Synthesized according to General Procedure A using 1,3-dioxoisoindolin-2-yl cyclohexanecarboxylate 1a $(54.5$ $\mathrm{mg}, 0.2 \mathrm{mmol}, 1$ equiv.) and phenyl vinyl sulfone $2 \mathbf{a}(50.4 \mathrm{mg}, 0.3 \mathrm{mmol}, 1.5$ equiv.). The crude mixture was purified by flash column chromatography on silica gel (5\% AcOEt in hexanes as eluent) to afford $\mathbf{3 a}$ ( $43.5 \mathrm{mg}, 86 \%$ yield) as a white solid.

${ }^{1} \mathrm{H}$ NMR $\left(500 \mathrm{MHz}, \mathrm{CDCl}_{3}\right) \delta 7.94-7.85(\mathrm{~m}, 2 \mathrm{H}), 7.69-7.61(\mathrm{~m}, 1 \mathrm{H}), 7.61-7.52(\mathrm{~m}, 2 \mathrm{H})$, $3.13-3.05(\mathrm{~m}, 2 \mathrm{H}), 1.71-1.54(\mathrm{~m}, 7 \mathrm{H}), 1.28(\mathrm{ddt}, J=14.6,7.5,3.8 \mathrm{~Hz}, 1 \mathrm{H}), 1.23-1.06(\mathrm{~m}$, $3 \mathrm{H}), 0.92-0.76(\mathrm{~m}, 2 \mathrm{H})$.

$\underline{{ }^{13} \mathrm{C} \mathrm{NMR}}\left(126 \mathrm{MHz}, \mathrm{CDCl}_{3}\right) \delta 139.4,133.7,129.4,128.2,54.5,36.8,32.9,29.7,26.4,26.1$.

Matching reported literature data. ${ }^{15}$ 
$\widehat{\sim} \mathrm{CN}$

6-phenylhexanenitrile (3b): Synthesized according to General Procedure A using 5 equiv. of $\gamma$-terpinene, 1,3-dioxoisoindolin-2-yl 4-phenylbutanoate $\mathbf{1 b}$

(62 mg, $0.2 \mathrm{mmol}, 1$ equiv.) and acrylonitrile $\mathbf{2 b}(26.3 \mu \mathrm{L}, 0.4 \mathrm{mmol}, 2$ equiv.). The crude mixture was purified by flash column chromatography on silica gel (5\% AcOEt in hexanes as eluent) to afford $\mathbf{3 b}$ (21 $\mathrm{mg}, 61 \%$ yield) as a yellow oil.

${ }^{1} \mathrm{H}$ NMR $\left(400 \mathrm{MHz}, \mathrm{CDCl}_{3}\right) \delta 7.33-7.24(\mathrm{~m}, 2 \mathrm{H}), 7.23-7.14(\mathrm{~m}, 3 \mathrm{H}), 2.64(\mathrm{t}, J=7.6 \mathrm{~Hz}, 2 \mathrm{H})$, $2.33(\mathrm{t}, J=7.1 \mathrm{~Hz}, 2 \mathrm{H}), 1.75-1.62(\mathrm{~m}, 4 \mathrm{H}), 1.54-1.42(\mathrm{~m}, 2 \mathrm{H})$.

${ }^{13} \mathrm{C} \mathrm{NMR}\left(101 \mathrm{MHz}, \mathrm{CDCl}_{3}\right) \delta 142.1,128.5,128.5,126.0,119.9,35.7,30.7,28.4,25.4,17.2$.

Matching reported literature data. ${ }^{16}$<smiles>c1ccc(OCCCC2CCCN2)cc1</smiles>

\section{Benzyl 2-(2-(phenylsulfonyl)ethyl)pyrrolidine-1-carboxylate}

(3h): Synthesized according to General Procedure A using 1-benzyl 2-(1,3dioxoisoindolin-2-yl) pyrrolidine-1,2-dicarboxylate 1c $(54.5 \mathrm{mg}, 0.2 \mathrm{mmol}, 1$ equiv.) and phenyl vinyl sulfone $2 \mathrm{a}(50.4 \mathrm{mg}, 0.3 \mathrm{mmol}, 1.5$ equiv.). The crude mixture was purified by flash column chromatography on silica gel (20\% AcOEt in hexanes as eluent) to afford 3h (56 mg, 75\% yield) as a white solid.

${ }^{1} \mathrm{H}$ NMR $\left(400 \mathrm{MHz}, \mathrm{CDCl}_{3}\right)$ mixture of rotamers: $\delta 7.96-7.77(\mathrm{~m}, 2 \mathrm{H}), 7.68-7.60(\mathrm{~m}, 1 \mathrm{H})$, $7.54(\mathrm{~d}, J=7.5 \mathrm{~Hz}, 2 \mathrm{H}), 7.40-7.20(\mathrm{~m}, 5 \mathrm{H}), 5.05(\mathrm{~d}, J=7.6 \mathrm{~Hz}, 2 \mathrm{H}), 3.94(\mathrm{~d}, J=9.9 \mathrm{~Hz}, 1 \mathrm{H})$, $3.58-3.28(\mathrm{~m}, 2 \mathrm{H}), 3.27-2.91(\mathrm{~m}, 2 \mathrm{H}), 2.23-1.74(\mathrm{~m}, 5 \mathrm{H}), 1.63(\mathrm{ddd}, J=11.3,5.5,3.0 \mathrm{~Hz}$, $1 \mathrm{H})$.

${ }^{13} \mathrm{C}$ NMR $\left(101 \mathrm{MHz}, \mathrm{CDCl}_{3}\right)$ mixture of rotamers: $\delta 155.5,139.3,136.9,133.8,129.4,128.6$, $128.1,127.9,67.2,66.9,56.5,55.9,54.0,53.7,46.9,46.5,31.2,30.7,27.9,23.8,23.1$.

Matching reported literature data. ${ }^{17}$

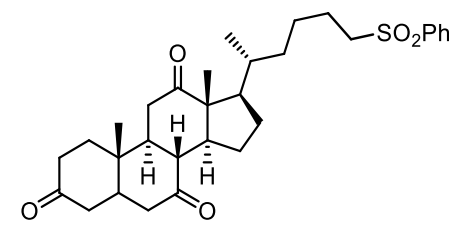

$(5 S, 8 R, 9 S, 10 S, 13 R, 14 S, 17 R)-10,13-d i m e t h y l-17-(6-$ (phenylsulfonyl)hexan-2-yl)dodecahydro-3Hcyclopenta[a]phenanthrene-3,7,12(2H,4H)-trione $\quad(3 \mathrm{k})$ : Synthesized according to General Procedure A using 1,3dioxoisoindolin-2-yl $\quad 4-((5 S, 8 R, 9 S, 10 S, 13 R, 14 S, 17 R)-10,13-$ dimethyl-3,7,12-trioxohexadecahydro-1H-cyclopenta[a]

phenanthren-17-yl)pentanoate $\mathbf{1 d}$ ( $54.5 \mathrm{mg}, 0.2 \mathrm{mmol}, 1$ equiv.) and phenyl vinyl sulfone 2a (50.4 $\mathrm{mg}, 0.3 \mathrm{mmol}, 1.5$ equiv.). The crude mixture was purified by flash column chromatography on silica gel (50\% AcOEt in hexanes as eluent) to afford $3 \mathbf{k}(48.0 \mathrm{mg}, 46 \%$ yield) as a white solid.

${ }^{1} \mathrm{H} \mathrm{NMR}\left(400 \mathrm{MHz}, \mathrm{CDCl}_{3}\right) \delta 7.95-7.85(\mathrm{~m}, 2 \mathrm{H}), 7.70-7.61(\mathrm{~m}, 1 \mathrm{H}), 7.61-7.53(\mathrm{~m}, 2 \mathrm{H})$, $3.16-3.00(\mathrm{~m}, 2 \mathrm{H}), 2.95-2.78(\mathrm{~m}, 3 \mathrm{H}), 2.38-2.17(\mathrm{~m}, 6 \mathrm{H}), 2.16-2.06(\mathrm{~m}, 2 \mathrm{H}), 2.02-1.90$ $(\mathrm{m}, 4 \mathrm{H}), 1.88-1.52(\mathrm{~m}, 5 \mathrm{H}), 1.47-1.30(\mathrm{~m}, 1 \mathrm{H}), 1.39(\mathrm{~s}, 3 \mathrm{H}), 1.32-1.12(\mathrm{~m}, 5 \mathrm{H}), 1.04(\mathrm{~s}, 3 \mathrm{H})$, $0.78(\mathrm{~d}, J=6.6 \mathrm{~Hz}, 3 \mathrm{H})$.

${ }^{13} \mathrm{C}$ NMR $\left(101 \mathrm{MHz}, \mathrm{CDCl}_{3}\right) \delta 212.1,209.1,208.9,139.4,133.8,129.4,128.2,57.0,56.5,51.9$, 49.1, 47.0, 45.8, 45.7, 45.1, 42.9, 38.8, 36.6, 36.2, 35.9, 35.4, 34.9, 28.0, 25.4, 25.3, 23.1, 22.0, 19.0, 12.0 .

Matching reported literature data. ${ }^{15}$ 
C2.3 General Procedure B (one-pot telescoped from carboxylic acids)

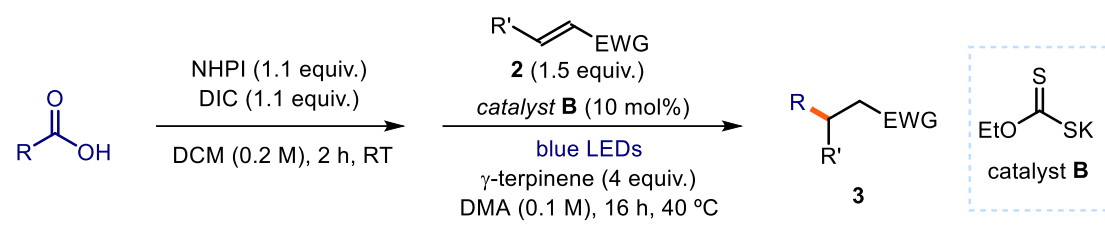

Reactions performed using set-up 1 in Figure S2. In an oven dried vial with a Teflon septum screw cap, carboxylic acid ( $0.2 \mathrm{mmol}, 1$ equiv.) and $N$-hydroxyphthalimide (NHPI, $35.8 \mathrm{mg}, 0.22$ mmol, 1.1 equiv.) were dissolved in $\mathrm{CH}_{2} \mathrm{Cl}_{2}$ (1 $\mathrm{mL}$, HPLC grade) and $N, N^{\prime}$ diisopropylcarbodiimide (DIC, $34 \mu \mathrm{L}, 0.22 \mathrm{mmol}, 1.1$ equiv.) was added via syringe. The reaction was stirred at ambient temperature until complete consumption of the carboxylic acid was observed by TLC (usually 1-2 hours). The crude reaction mixture was concentrated under vacuum to obtain the crude phthalimide ester, which was used without further purification in the next step.

In the same vial containing the crude phthalimide ester, xanthogenate B ( $3.2 \mathrm{mg}, 0.02 \mathrm{mmol}, 0.1$ equiv.) and the electron-poor olefin 2 ( $0.3 \mathrm{mmol}, 1.5$ equiv., if solid $)$ were dissolved in DMA (2 $\mathrm{mL}$, synthesis grade). Next, $\gamma$-terpinene $(128 \mu \mathrm{L}, 0.8 \mathrm{mmol}, 4$ equiv.) was added and the resulting orange mixture was degassed with argon sparging for 60 seconds. If the electron-poor olefin 2 was liquid, it was added via syringe after the argon sparging. The vial was then placed in the 3D printed support photoreactor (Figure S2) and irradiated under stirring for 16 hours, if not otherwise specified. The mixture was transferred to an extraction funnel, $\mathrm{NaOH} 1 \mathrm{M}$ solution was added and the organic layer was extracted with $\mathrm{CH}_{2} \mathrm{Cl}_{2}$. The organic layer was washed with brine twice. The combined organic layers were dried over anhydrous $\mathrm{MgSO}_{4}$, filtered, and concentrated to dryness. The crude residue was purified by column chromatography to afford the corresponding product in the stated yield with $>95 \%$ purity according to ${ }^{1} \mathrm{H}$ NMR analysis.

\section{C2.4 Characterization of Products with General Procedure B}

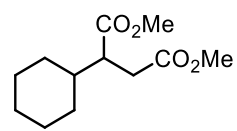

dimethyl 2-cyclohexylsuccinate (3c): Synthesized according to General Procedure B using 2 equiv. of $\gamma$-terpinene, 1,3-dioxoisoindolin-2-yl cyclohexanecarboxylate 1a $(54.5 \mathrm{mg}, 0.2 \mathrm{mmol}, 1$ equiv.) and dimethyl fumarate $2 \mathrm{c}$ ( $43 \mathrm{mg}, 0.3 \mathrm{mmol}, 1.5$ equiv.). The crude mixture was purified by flash column chromatography on silica gel (5\% AcOEt in hexanes as eluent) to afford $\mathbf{3 c}$ (44 $\mathrm{mg}, 95 \%$ yield) as a yellow oil.

${ }^{1} \mathrm{H}$ NMR $\left(500 \mathrm{MHz}, \mathrm{CDCl}_{3}\right) \delta 3.69(\mathrm{~s}, 3 \mathrm{H}), 3.66(\mathrm{~s}, 3 \mathrm{H}), 2.78-2.66(\mathrm{~m}, 2 \mathrm{H}), 2.45(\mathrm{dt}, J=13.1$, $8.9 \mathrm{~Hz}, 1 \mathrm{H}), 1.78-1.69(\mathrm{~m}, 2 \mathrm{H}), 1.69-1.52(\mathrm{~m}, 4 \mathrm{H}), 1.31-1.15(\mathrm{~m}, 2 \mathrm{H}), 1.15-1.07(\mathrm{~m}, 1 \mathrm{H})$, $1.06-0.92(\mathrm{~m}, 2 \mathrm{H})$.

${ }^{13} \mathrm{C} \mathrm{NMR}\left(126 \mathrm{MHz}, \mathrm{CDCl}_{3}\right) \delta 175.1,173.1,51.9,51.7,47.2,40.1,33.4,30.8,30.3,26.4,26.3$.

Matching reported literature data. ${ }^{15}$<smiles>CC(C)(C)CC[Se][OH+]c1ccccc1</smiles>

((3,3-dimethylbutyl)sulfonyl)benzene (3d): Synthesized according to General Procedure B using pivalic acid (20.4 mg, $0.2 \mathrm{mmol}, 1$ equiv.) and phenyl vinyl sulfone $2 \mathrm{a}$ (50.4 $\mathrm{mg}, 0.3 \mathrm{mmol}, 1.5$ equiv.). The crude mixture was purified by flash column chromatography on silica gel (7\% AcOEt in hexanes as eluent) to afford $\mathbf{3 d}$ (33.0 $\mathrm{mg}, 73 \%$ yield) as a yellow oil.

${ }^{1} \mathrm{H}$ NMR $\left(400 \mathrm{MHz}, \mathrm{CDCl}_{3}\right) \delta 7.98-7.85(\mathrm{~m}, 2 \mathrm{H}), 7.71-7.62(\mathrm{~m}, 1 \mathrm{H}), 7.65-7.53(\mathrm{~m}, 2 \mathrm{H})$, $3.10-3.01(\mathrm{~m}, 2 \mathrm{H}), 1.64-1.55(\mathrm{~m}, 2 \mathrm{H}), 0.86(\mathrm{~s}, 9 \mathrm{H})$.

${ }^{13} \mathrm{C} \mathrm{NMR}\left(101 \mathrm{MHz}, \mathrm{CDCl}_{3}\right) \delta 142.1,128.5,128.5,126.0,119.9,35.7,30.7,28.4,25.4,17.2$.

Matching reported literature data. ${ }^{15}$ 


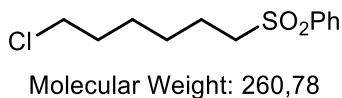

((7-chloroheptyl)sulfonyl)benzene (3e): Synthesized according to General Procedure B using 5-Chlorovaleric acid(27.3 mg, $0.2 \mathrm{mmol}, 1.0$ equiv.) and (vinylsulfonyl)benzene ( $50.5 \mathrm{mg}, 0.3 \mathrm{mmol}, 1.5$ equiv.). The crude mixture was purified by flash column chromatography on silica gel (20\% DCM in hexanes) to afford $3 \mathbf{e}(29.1 \mathrm{mg}, 56 \%$ yield) as a white solid.

${ }^{1} \mathrm{H} \mathrm{NMR}\left(400 \mathrm{MHz}, \mathrm{CDCl}_{3}\right) \delta 7.95-7.88(\mathrm{~m}, 2 \mathrm{H}), 7.69-7.64(\mathrm{~m}, 1 \mathrm{H}), 7.61-7.55(\mathrm{~m}, 2 \mathrm{H})$, $3.50(\mathrm{t}, J=6.5 \mathrm{~Hz}, 2 \mathrm{H}), 3.12-3.05(\mathrm{~m}, 2 \mathrm{H}), 1.77-1.71(\mathrm{~m}, 4 \mathrm{H}), 1.44-1.39(\mathrm{~m}, 4 \mathrm{H})$.

${ }^{13} \mathrm{C} \mathrm{NMR}\left(101 \mathrm{MHz}, \mathrm{CDCl}_{3}\right) \delta 139.3,133.8,129.4,128.2,56.3,44.9,32.2,29.8,27.7,26.4,22.7$.

Matching reported literature data. ${ }^{18}$

C2.5 General Procedure C (one-pot domino from carboxylic acids)

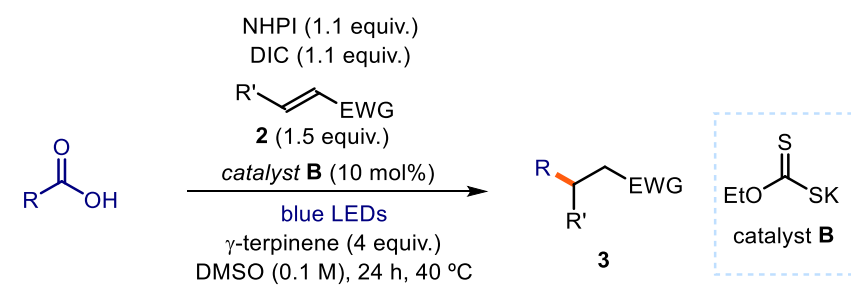

Reactions performed using set-up 1 in Figure S2. In an oven dried vial with a Teflon septum screw cap, carboxylic acid ( $0.2 \mathrm{mmol}, 1$ equiv.), $N$-hydroxyphthalimide (NHPI, $35.8 \mathrm{mg}, 0.22$ mmol, 1.1 equiv.), xanthogenate catalyst $\mathbf{B}(3.2 \mathrm{mg}, 0.02 \mathrm{mmol}, 0.1$ equiv.), and the electron-poor olefin 2 (0.3 mmol, 1.5 equiv., if solid) were dissolved in DMSO (2 mL) and $N, N^{\prime}-$ diisopropylcarbodiimide (DIC, $34 \mu \mathrm{L}, 0.22 \mathrm{mmol}, 1.1$ equiv.) was added via syringe. Next, $\gamma$ terpinene ( $128 \mu \mathrm{L}, 0.8 \mathrm{mmol}, 4$ equiv.) was added and the resulting orange mixture was degassed with argon sparging for 60 seconds. If the electron-poor olefin 2 were liquid, it was added via syringe after the argon sparging. The vial was then placed in the 3D printed support photoreactor (Figure S2) and irradiated under stirring for 24 hours, unless otherwise specified. The mixture was transferred to an extraction funnel, $\mathrm{NaOH} 1 \mathrm{M}$ solution was added and the organic layer was extracted with $\mathrm{CH}_{2} \mathrm{Cl}_{2}$. The organic layer was washed with brine twice. The combined organic layers were dried over anhydrous $\mathrm{MgSO}_{4}$, filtered, and concentrated to dryness. The crude residue was purified by column chromatography to afford the corresponding product in the stated yield with $>95 \%$ purity according to ${ }^{1} \mathrm{H}$ NMR analysis.

\section{C2.6 Characterization of Products with General Procedure C}<smiles>CC(C)C(N)CC[SeH2+][OH+]</smiles>

tert-butyl (4-methyl-1-(phenylsulfonyl)pentan-3-yl)carbamate

(3f): Synthesized according to General Procedure C using NMP as solvent, L-valine (43.5 mg, $0.2 \mathrm{mmol}, 1$ equiv.) and phenyl vinyl sulfone $2 \mathrm{a}(50.4 \mathrm{mg}, 0.3 \mathrm{mmol}$, 1.5 equiv.). The crude mixture was purified by flash column chromatography on silica gel (25\% AcOEt in hexanes as eluent) to afford $\mathbf{3 f}$ ( $63 \mathrm{mg}, 92 \%$ yield) as a white solid.

${ }^{1} \mathrm{H} \mathrm{NMR}\left(500 \mathrm{MHz}, \mathrm{CDCl}_{3}\right) \delta 7.93-7.88(\mathrm{~m}, 2 \mathrm{H}), 7.69-7.63(\mathrm{~m}, 1 \mathrm{H}), 7.57(\mathrm{dd}, J=8.4,7.1$ $\mathrm{Hz}, 2 \mathrm{H}), 4.32(\mathrm{~d}, J=10.1 \mathrm{~Hz}, 1 \mathrm{H}), 3.42(\mathrm{td}, J=10.4,4.8 \mathrm{~Hz}, 1 \mathrm{H}), 3.15(\mathrm{ddd}, J=9.1,6.1,1.7 \mathrm{~Hz}$, 2H), 1.93 (tdd, $J=12.1,7.4,3.4 \mathrm{~Hz}, 1 \mathrm{H}), 1.82-1.61(\mathrm{~m}, 2 \mathrm{H}), 1.40(\mathrm{~s}, 9 \mathrm{H}), 0.87$ (dd, $J=10.0$, $6.8 \mathrm{~Hz}, 6 \mathrm{H})$.

${ }^{13} \mathrm{C}$ NMR $\left(126 \mathrm{MHz}, \mathrm{CDCl}_{3}\right) \delta 156.1,139.4,133.9,129.5,128.1,79.7,54.7,54.1,32.8,28.5$, 25.9, 19.2, 17.8.

Matching reported literature data. ${ }^{19}$ 
<smiles>CC(C)C(N)CCCN</smiles>

tert-butyl (1-cyano-4-methylpentan-3-yl)carbamat according to General Procedure $C$ using 5 equiv. of $\gamma$-terpinene, L-valine $(43.5 \mathrm{mg}$, $0.2 \mathrm{mmol}, 1$ equiv.) and acrylonitrile $\mathbf{2 b}(26.3 \mu \mathrm{L}, 0.4 \mathrm{mmol}, 2$ equiv.). The crude mixture was purified by flash column chromatography on silica gel (15\% AcOEt in hexanes as eluent) to afford $\mathbf{3 g}$ ( $27 \mathrm{mg}, 60 \%$ yield) as a yellow oil.

${ }^{1} \mathrm{H} \mathrm{NMR}\left(400 \mathrm{MHz}, \mathrm{CDCl}_{3}\right) \delta 4.34(\mathrm{~d}, J=9.9 \mathrm{~Hz}, 1 \mathrm{H}), 3.45(\mathrm{tdd}, J=10.4,5.5,3.4 \mathrm{~Hz}, 1 \mathrm{H}), 2.49$ $-2.30(\mathrm{~m}, 2 \mathrm{H}), 1.90(\mathrm{td}, J=11.7,9.8,5.5 \mathrm{~Hz}, 1 \mathrm{H}), 1.72(\mathrm{dt}, J=13.0,6.5 \mathrm{~Hz}, 1 \mathrm{H}), 1.62(\mathrm{td}, J=$ $15.0,14.5,9.7 \mathrm{~Hz}, 1 \mathrm{H}), 1.44(\mathrm{~s}, 9 \mathrm{H}), 0.91(\mathrm{dd}, J=10.4,6.8 \mathrm{~Hz}, 6 \mathrm{H})$.

${ }^{13} \mathrm{C} \mathrm{NMR}\left(101 \mathrm{MHz}, \mathrm{CDCl}_{3}\right) \delta 156.1,119.9,79.7,55.4,32.5,29.4,28.5,19.2,17.9,14.7$.

Matching reported literature data. ${ }^{19}$

Boc tert-butyl methyl(3-(phenylsulfonyl)propyl)carbamate (3i): Synthesized $-\mathrm{N} \sim \mathrm{SO}_{2} \mathrm{Ph}$ according to General Procedure $\mathrm{C}$ using $\mathrm{N}$-(tert-butoxycarbonyl)-Nmethylglycine (38 mg, $0.2 \mathrm{mmol}, 1$ equiv.) and phenyl vinyl sulfone $\mathbf{2 a}(50.4 \mathrm{mg}, 0.3 \mathrm{mmol}, 1.5$ equiv.). The crude mixture was purified by flash column chromatography on silica gel (20\% AcOEt in hexanes as eluent) to afford $\mathbf{3 i}$ (47 $\mathrm{mg}, 75 \%$ yield) as a yellow oil.

${ }^{1} \mathrm{H}$ NMR $\left(400 \mathrm{MHz}, \mathrm{CDCl}_{3}\right)$ mixture of rotamers: $\delta 7.94-7.86(\mathrm{~m}, 2 \mathrm{H}), 7.70-7.62(\mathrm{~m}, 1 \mathrm{H})$, $7.57(\mathrm{dd}, J=8.3,6.8 \mathrm{~Hz}, 2 \mathrm{H}), 3.29(\mathrm{t}, J=6.8 \mathrm{~Hz}, 2 \mathrm{H}), 3.11-2.99(\mathrm{~m}, 2 \mathrm{H}), 2.79(\mathrm{~s}, 3 \mathrm{H}), 1.99-$ $1.87(\mathrm{~m}, 2 \mathrm{H}), 1.40(\mathrm{~s}, 9 \mathrm{H})$.

${ }^{13} \mathrm{C} \mathrm{NMR}\left(101 \mathrm{MHz}, \mathrm{CDCl}_{3}\right)$ mixture of rotamers: $\delta 155.9,139.2,133.9,129.5,128.1,79.9,53.9$, 47.4, 34.3, 28.5, 21.0.

HRMS: calculated for $\mathrm{C}_{15} \mathrm{H}_{23} \mathrm{NNaO}_{4} \mathrm{~S}\left(\mathrm{M}+\mathrm{Na}^{+}\right)$: 336.1240 , found $336.1236(+1.2 \mathrm{ppm})$.

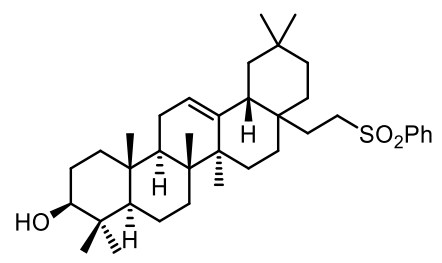

$(3 S, 4 \mathrm{a} R, 6 \mathrm{a} R, 6 \mathrm{~b} S, 12 \mathrm{a} R, 14 \mathrm{a} R, 14 \mathrm{~b} R)-4,4,6 \mathrm{a}, 6 \mathrm{~b}, 11,11,14 \mathrm{~b}-$ heptamethyl-8a-(2-(phenylsulfonyl)ethyl)-

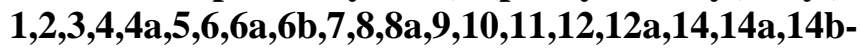
icosahydropicen-3-ol (3j): Synthesized according to General Procedure C using NMP as solvent, oleanolic acid $(91 \mathrm{mg}, 0.2$ mmol, 1 equiv) and phenyl vinyl sulfone 2 a $(50.4 \mathrm{mg}, 0.3 \mathrm{mmol}$, 1.5 equiv.). The crude mixture was purified by flash column chromatography on silica gel (40\% AcOEt in hexanes as eluent) to afford $\mathbf{3 j}$ ( $84 \mathrm{mg}, 72 \%$ yield) as light-yellow solid.

${ }^{1} \mathrm{H} \mathrm{NMR}\left(400 \mathrm{MHz}, \mathrm{CDCl}_{3}\right) \delta 7.90-7.84(\mathrm{~m}, 2 \mathrm{H}), 7.67-7.59(\mathrm{~m}, 1 \mathrm{H}), 7.58-7.50(\mathrm{~m}, 2 \mathrm{H})$, $5.15(\mathrm{t}, J=3.6 \mathrm{~Hz}, 1 \mathrm{H}), 3.19(\mathrm{dd}, J=10.7,4.8 \mathrm{~Hz}, 1 \mathrm{H}), 3.01(\mathrm{dtd}, J=37.9,13.5,4.3 \mathrm{~Hz}, 2 \mathrm{H})$, $2.01-1.76(\mathrm{~m}, 7 \mathrm{H}), 1.66(\mathrm{t}, J=13.5 \mathrm{~Hz}, 1 \mathrm{H}), 1.62-\mathrm{z} 1.36(\mathrm{~m}, 7 \mathrm{H}), 1.37-1.26(\mathrm{~m}, 3 \mathrm{H}), 1.23-$ $1.11(\mathrm{~m}, 4 \mathrm{H}), 1.09(\mathrm{~s}, 3 \mathrm{H}), 1.02(\mathrm{dd}, J=13.7,2.1 \mathrm{~Hz}, 1 \mathrm{H}), 0.97(\mathrm{~s}, 3 \mathrm{H}), 0.94-0.90(\mathrm{~m}, 1 \mathrm{H}), 0.88$ (s, 3H), 0.85 (s, 3H), 0.82 (s, 3H), 0.77 (s, 3H), $0.68(\mathrm{dd}, J=11.5,1.9 \mathrm{~Hz}, 1 \mathrm{H}), 0.60(\mathrm{~s}, 3 \mathrm{H})$.

${ }^{13} \mathrm{C} \mathrm{NMR}\left(101 \mathrm{MHz}, \mathrm{CDCl}_{3}\right) \delta 143.6,139.3,133.6,129.4,128.1,123.1,79.0,55.2,51.6,47.6$, $46.9,46.5,41.6,39.7,38.9,38.7,37.0,34.7,34.3,33.2$, 32.9, 32.5, 31.9, 31.0, 29.8, 28.2, 27.3, $26.1,25.5,23.6,23.3,18.4,16.5,15.7,15.6$.

Matching reported literature data. ${ }^{15}$

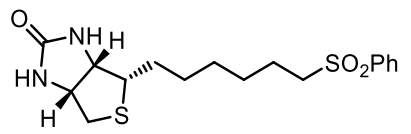

(3aS,4S,6aR)-4-(6-(phenylsulfonyl)hexyl)tetrahydro-1Hthieno[3,4-d]imidazol-2(3H)-one (31): Synthesized according to General Procedure C using $4 \mathrm{~mL}$ of DMSO, biotin $(49 \mathrm{mg}, 0.2 \mathrm{mmol}$, 1 equiv.) and phenyl vinyl sulfone $2 \mathbf{a}(50.4 \mathrm{mg}, 0.3 \mathrm{mmol}, 1.5$ equiv.). The crude mixture was purified by flash column chromatography on silica gel (2-5\% $\mathrm{MeOH}$ in DCM as eluent) to afford $\mathbf{3 j}$ ( $48 \mathrm{mg}, 65 \%$ yield) as a yellow oil. 
${ }^{1} \mathrm{H}$ NMR $\left(500 \mathrm{MHz}\right.$, Methanol- $\left.d_{4}\right) \delta 7.99-7.89(\mathrm{~m}, 2 \mathrm{H}), 7.77-7.70(\mathrm{~m}, 1 \mathrm{H}), 7.72-7.58(\mathrm{~m}$, 2H), $4.53-4.46(\mathrm{~m}, 1 \mathrm{H}), 4.29$ (ddd, $J=12.2,7.9,4.5 \mathrm{~Hz}, 1 \mathrm{H}), 3.24-3.19(\mathrm{~m}, 2 \mathrm{H}), 3.19-3.14$ (m, 1H), $2.92(\mathrm{dt}, J=12.7,5.1 \mathrm{~Hz}, 1 \mathrm{H}), 2.70(\mathrm{dd}, J=12.7,4.4 \mathrm{~Hz}, 1 \mathrm{H}), 1.66(\mathrm{tdd}, J=15.3,8.3$, $4.4 \mathrm{~Hz}, 3 \mathrm{H}), 1.53$ (ddd, $J=16.8,8.8,5.5 \mathrm{~Hz}, 1 \mathrm{H}), 1.45-1.27$ (m, 6H).

${ }^{13} \mathrm{C}$ NMR $\left(101 \mathrm{MHz}, \mathrm{CDCl}_{3}\right) \delta 164.5,139.2,133.8,129.4,128.1,62.4,60.8,56.1,55.8,40.6$, 31.4, 31.0, 28.8, 28.7, 28.4, 27.9, 22.7, 22.5, 14.1.

HRMS: calculated for $\mathrm{C}_{17} \mathrm{H}_{24} \mathrm{~N}_{2} \mathrm{NaO}_{3} \mathrm{~S}_{2}\left(\mathrm{M}+\mathrm{Na}^{+}\right)$: 391.1120, found 391.1121 ( $\left.-0.3 \mathrm{ppm}\right)$.

\section{C2.7 General Procedure D (using pyridinium salts)}
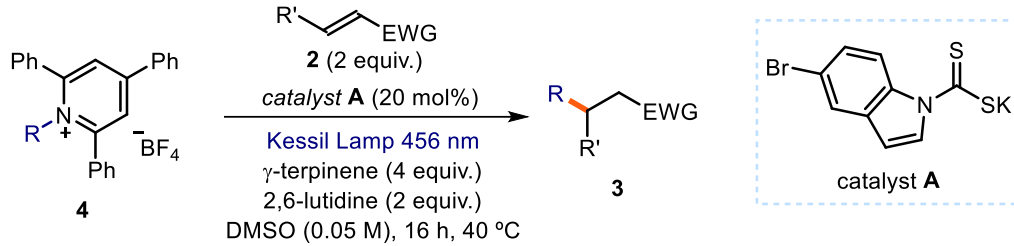

Reactions performed using set-up 2 in Figure S3. In an oven dried vial, with a Teflon septum screw cap, dithiocarbamate A (12.4 mg, $0.04 \mathrm{mmol}, 0.2$ equiv.), pyridinium salt 4 (0.2 mmol, 1 equiv.) and the electron-poor olefin 2 (0.4 mmol, 2 equiv.), were dissolved in DMSO (4 mL). Then, $\gamma$-terpinene ( $128 \mu \mathrm{L}, 0.8 \mathrm{mmol}, 4$ equiv.) and 2,6-lutidine ( $46 \mu \mathrm{L}, 0.4 \mathrm{mmol}, 2$ equiv.) were added. The resulting orange mixture was degassed with argon sparging for 60 seconds. The vial was then placed at 2-3 cm of a $50 \mathrm{~W}$ Kessil blue LED lamp and irradiated under stirring for 16 hours (see Figure S3). The mixture was transferred to an extraction funnel, $\mathrm{NaHCO}_{3}$ sat. solution was added and the organic layer was extracted with EtOAc. The organic layer was washed with brine twice. The combined organic layers were dried over anhydrous $\mathrm{MgSO}_{4}$, filtered, and concentrated to dryness. The crude residue was purified by column chromatography to afford the corresponding product in the stated yield with $>95 \%$ purity according to ${ }^{1} \mathrm{H}$ NMR analysis.

\section{C2.8 Characterization of Products with General Procedure D}

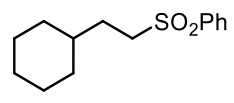

((2-cyclohexylethyl)sulfonyl)benzene (3a): Synthesized according to General Procedure D using 1-cyclohexyl-2,4,6-triphenylpyridin-1-ium tetrafluoroborate 4a (95 mg, $0.2 \mathrm{mmol}, 1$ equiv.) and phenyl vinyl sulfone $\mathbf{2 a}(67 \mathrm{mg}, 0.4 \mathrm{mmol}$, 2 equiv.). The crude mixture was purified by flash column chromatography on silica gel $(10 \%$ AcOEt in hexanes as eluent) to afford $\mathbf{3 a}$ (34 mg, 67\% yield) as a white solid.

${ }^{1} \mathrm{H}$ NMR $\left(500 \mathrm{MHz}, \mathrm{CDCl}_{3}\right) \delta 7.94-7.85(\mathrm{~m}, 2 \mathrm{H}), 7.69-7.61(\mathrm{~m}, 1 \mathrm{H}), 7.61-7.52(\mathrm{~m}, 2 \mathrm{H})$, $3.13-3.05(\mathrm{~m}, 2 \mathrm{H}), 1.71-1.54(\mathrm{~m}, 7 \mathrm{H}), 1.28(\mathrm{ddt}, J=14.6,7.5,3.8 \mathrm{~Hz}, 1 \mathrm{H}), 1.23-1.06(\mathrm{~m}$, $3 \mathrm{H}), 0.92-0.76(\mathrm{~m}, 2 \mathrm{H})$.

${ }^{13} \mathrm{C} \mathrm{NMR}\left(126 \mathrm{MHz}, \mathrm{CDCl}_{3}\right) \delta 139.4,133.7,129.4,128.2,54.5,36.8,32.9,29.7,26.4,26.1$.

Matching reported literature data. ${ }^{15}$

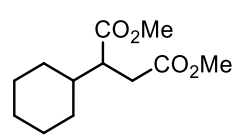

dimethyl 2-cyclohexylsuccinate (3c): Synthesized according to General Procedure D using 2 equiv. of $\gamma$-terpinene, 1-cyclohexyl-2,4,6-triphenylpyridin1-ium tetrafluoroborate $\mathbf{4 a}$ ( $95 \mathrm{mg}, 0.2 \mathrm{mmol}, 1$ equiv.) and dimethyl fumarate 2c (57 mg, $0.4 \mathrm{mmol}, 2$ equiv.). The crude mixture was purified by flash column chromatography on silica gel (5\% AcOEt in hexanes as eluent) to afford 3c (41 mg, 90\% yield) as a yellow oil.

${ }^{1} \mathrm{H}$ NMR $\left(400 \mathrm{MHz}, \mathrm{CDCl}_{3}\right) \delta 3.69(\mathrm{~s}, 3 \mathrm{H}), 3.66(\mathrm{~s}, 3 \mathrm{H}), 2.78-2.66(\mathrm{~m}, 2 \mathrm{H}), 2.45$ (dt, $J=13.1$, $8.9 \mathrm{~Hz}, 1 \mathrm{H}), 1.78-1.69(\mathrm{~m}, 2 \mathrm{H}), 1.69-1.52(\mathrm{~m}, 4 \mathrm{H}), 1.31-1.15(\mathrm{~m}, 2 \mathrm{H}), 1.15-1.07(\mathrm{~m}, 1 \mathrm{H})$, $1.06-0.92(\mathrm{~m}, 2 \mathrm{H})$.

${ }^{13} \mathrm{C} \mathrm{NMR}\left(126 \mathrm{MHz}, \mathrm{CDCl}_{3}\right) \delta 175.1,173.1,51.9,51.7,47.2,40.1,33.4,30.8,30.3,26.4,26.3$.

Matching reported literature data. ${ }^{15}$ 
<smiles>COC(=O)C1CC(=O)OCC1Cc1ccccc1</smiles>

$3 m$

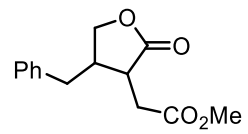

$3 m^{\prime}$

methyl 5-benzyl-2-oxotetrahydro-2H-pyran-4-carboxylate (3m): Synthesized according to General Procedure D using 2 equiv. of $\gamma$-terpinene, 1-(1-hydroxy-3-phenylpropan-2-yl)2,4,6-triphenylpyridin-1-ium tetrafluoroborate $\mathbf{4 b}$ (106 $\mathrm{mg}, 0.2$ mmol, 1 equiv.) and dimethyl fumarate $2 \mathbf{c}(57 \mathrm{mg}, 0.4 \mathrm{mmol}, 2$ equiv.). The crude mixture was purified by flash column chromatography on silica gel $(10 \%$ AcOEt in hexanes as eluent) to afford an unseparable mixture of regiosiomers $\mathbf{3 m} / \mathbf{3} \mathbf{m}$ ' (3.8:1) (27 mg, 54\% yield) as a yellow oil. Corrected yield for $3 \mathrm{~m}$ ( $42 \%$ yield)

Products formed upon intramolecular esterification promoted by the acidic conditions delivered by the silica used for purification.

${ }^{1} \mathrm{H}$ NMR $\left(400 \mathrm{MHz}, \mathrm{CDCl}_{3}\right)$ mixture of regiosiomers $\delta 7.35-7.28(\mathrm{~m}, 2.5 \mathrm{H}), 7.28-7.21(\mathrm{~m}$, $1.25 \mathrm{H}), 7.19-7.10(\mathrm{~m}, 2.5 \mathrm{H}), 4.35-4.28(\mathrm{~m}, 1 \mathrm{H}), 4.14-4.06(\mathrm{~m}, 0.5 \mathrm{H}), 3.96-3.88(\mathrm{~m}, 1 \mathrm{H})$, $3.74(\mathrm{~s}, 0.75 \mathrm{H}), 3.69(\mathrm{~s}, 3 \mathrm{H}), 3.31-3.23(\mathrm{~m}, 0.25 \mathrm{H}), 3.05-2.85(\mathrm{~m}, 1.5 \mathrm{H}), 2.85-2.66(\mathrm{~m}$, $3.25 \mathrm{H}), 2.63(\mathrm{~d}, J=10.2 \mathrm{~Hz}, 0.25 \mathrm{H}), 2.61-2.55(\mathrm{~m}, 2 \mathrm{H}), 2.34(\mathrm{dd}, J=13.7,11.9 \mathrm{~Hz}, 0.25 \mathrm{H})$.

${ }^{13} \mathrm{C} \mathrm{NMR}\left(101 \mathrm{MHz}, \mathrm{CDCl}_{3}\right)$ mixture of regiosiomers: $\delta$ 177.8, 171.6, 137.8, 129.1, 129.0, 129.0, $128.7,127.1,126.9,71.4,70.1,52.3,52.2,42.4,42.0,40.8,39.7,38.4,33.6,33.3,30.2,29.8$.

HRMS: calculated for $\mathrm{C}_{14} \mathrm{H}_{16} \mathrm{NaO}_{4}\left(\mathrm{M}+\mathrm{Na}^{+}\right)$: 271.0941, found 271.0939 (+0.7 ppm).

C2.9 General Procedure E (telescoped reaction from amine 5)
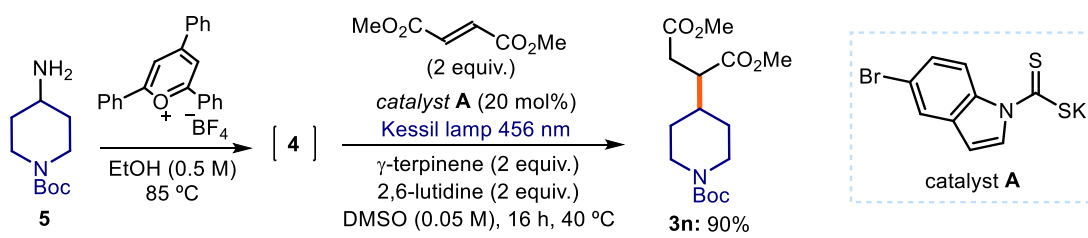

Reactions performed using set-up 2 in Figure S3. In an oven dried vial, with a Teflon septum screw cap, primary amine ( $0.22 \mathrm{mmol}, 1.1$ equiv.) and 2,4,6-triphenylpyrylium tetrafluoroborate (79. $2 \mathrm{mg}, 0.2 \mathrm{mmol}, 1$ equiv.) were dissolved in ethanol (0.4 mL, HPLC grade). The reaction was stirred at $80^{\circ} \mathrm{C}$ for 4 hours. In the same vial without evaporating the solvent, dithiocarbamate $\mathbf{A}$ (12.4 mg, $0.04 \mathrm{mmol}, 0.2$ equiv.), electron-poor olefin 2 ( $0.4 \mathrm{mmol}, 2$ equiv.), DMSO (4 mL), $\gamma$ terpinene $(64 \mu \mathrm{L}, 0.4$ mmol, 2 equiv.) and 2,6-lutidine ( $46 \mu \mathrm{L}, 0.4$ mmol, 2 equiv.) were added sequentially. The resulting orange mixture was degassed with argon sparging for 60 seconds. The vial was then placed at $2-3 \mathrm{~cm}$ of a $50 \mathrm{~W}$ Kessil blue LED lamp and irradiated under stirring for 16 hours (see Figure S3). The mixture was transferred to an extraction funnel, saturated aqueous $\mathrm{NaHCO}_{3}$ was added, and the organic layer extracted with EtOAc. The organic layer was washed with brine twice. The combined organic layers were dried over anhydrous $\mathrm{MgSO}_{4}$, filtered, and concentrated to dryness. The crude residue was purified by column chromatography to afford the corresponding product in the stated yield with $>95 \%$ purity according to ${ }^{1} \mathrm{H}$ NMR analysis.

A control experiment without catalyst $\mathbf{A}$ delivered no conversion of the pyridinium salt upon irradiation.

\section{C2.10 Characterization of Products with General Procedure E}<smiles>CC(=O)CC(COC(C)=O)C1CCNCC1</smiles>

dimethyl 2-(1-(tert-butoxycarbonyl)piperidin-4-yl)succinate (3n): Synthesized according to General Procedure E using 2 equiv. of $\gamma$-terpinene, tert-butyl 4-aminopiperidine-1-carboxylate ( $40 \mathrm{mg}, 0.2 \mathrm{mmol}, 1$ equiv.) and dimethyl fumarate $2 \mathrm{c}(57 \mathrm{mg}, 0.4 \mathrm{mmol}, 2$ equiv.). The crude mixture was purified by flash column chromatography on silica gel (0-40\% AcOEt in hexanes as eluent) to afford $\mathbf{3 n}(59 \mathrm{mg}$, $90 \%$ yield) as a yellow oil.

$\underline{{ }^{1} \mathrm{H} \mathrm{NMR}}\left(400 \mathrm{MHz}, \mathrm{CDCl}_{3}\right) \delta 4.12(\mathrm{~d}, J=13.3 \mathrm{~Hz}, 2 \mathrm{H}), 3.70(\mathrm{~s}, 3 \mathrm{H}), 3.66(\mathrm{~s}, 3 \mathrm{H}), 2.81-2.68$ (m, 2H), 2.63 (tt, $J=13.0,3.0 \mathrm{~Hz}, 2 \mathrm{H}), 2.52-2.41(\mathrm{~m}, 1 \mathrm{H}), 1.73$ (tdd, $J=12.3,6.1,3.6 \mathrm{~Hz}, 1 \mathrm{H})$, 1.57 (ddt, $J=23.3,13.0,3.0 \mathrm{~Hz}, 2 \mathrm{H}), 1.44$ (s, 9H), $1.34-1.15(\mathrm{~m}, 2 \mathrm{H})$. 
${ }^{13} \mathrm{C} \mathrm{NMR}\left(126 \mathrm{MHz}, \mathrm{CDCl}_{3}\right) \delta 174.7,172.9,155.1,79.9,52.2,46.6,44.2,38.7,33.6,33.6,29.9$, 29.7, 28.8, 28.8.

HRMS: calculated for $\mathrm{C}_{16} \mathrm{H}_{27} \mathrm{NNaO}_{6}\left(\mathrm{M}+\mathrm{Na}^{+}\right)$: 352.1731 , found 352.1726. (+1.4 ppm)

\section{C3. Reduction}

C3.1 General Procedure F (Barton decarboxylation)

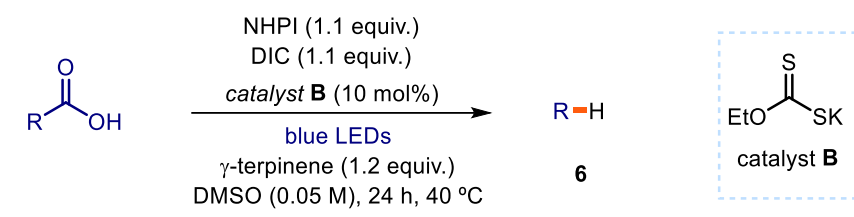

Reactions performed using set-up 1 in Figure S2. In an oven dried vial, with a Teflon septum screw cap, carboxylic acid ( $0.2 \mathrm{mmol}, 1$ equiv.), $N$-hydroxyphthalimide (NHPI, $35.8 \mathrm{mg}, 0.22$ mmol, 1.1 equiv.) and xanthogenate $\mathbf{B}(3.2 \mathrm{mg}, 0.02 \mathrm{mmol}, 0.1$ equiv.) were dissolved in DMSO (4 mL) and $N, N^{\prime}$-diisopropylcarbodiimide (DIC, $34 \mu \mathrm{L}, 0.22 \mathrm{mmol}, 1.1$ equiv.) was added via syringe. Then, $\gamma$-terpinene ( $38 \mu \mathrm{L}, 0.24 \mathrm{mmol}, 1.2$ equiv.) was added. The resulting orange mixture was degassed with argon sparging for 60 seconds. The vial was then placed in the 3D printed support photoreactor (Figure S2) and irradiated under stirring for 24 hours, unless otherwise specified. The mixture was transferred to an extraction funnel, $\mathrm{NaOH} 1 \mathrm{M}$ solution was added and the organic layer was extracted with $\mathrm{CH}_{2} \mathrm{Cl}_{2}$. The organic layer was washed with brine twice. The combined organic layers were dried over anhydrous $\mathrm{MgSO}_{4}$, filtered, and concentrated to dryness. The crude residue was purified by column chromatography to afford the corresponding product in the stated yield with $>95 \%$ purity according to ${ }^{1} \mathrm{H}$ NMR analysis.

\section{C3.2 Characterization of Products with General Procedure F}

NHBoc benzyl (S)-2-((tert-butoxycarbonyl)amino)butanoate (6a): Synthesized according to General Procedure $\mathrm{F}$ using (S)-5-(benzyloxy)-4-((tertbutoxycarbonyl)amino)-5-oxopentanoic acid (67 mg, $0.2 \mathrm{mmol}, 1$ equiv.). The crude mixture was purified by flash column chromatography on silica gel (25\% AcOEt in hexanes as eluent) to afford $\mathbf{6 a}(38 \mathrm{mg}, 65 \%$ yield) as a yellow oil.

${ }^{1} \mathrm{H} \mathrm{NMR}\left(400 \mathrm{MHz}, \mathrm{CDCl}_{3}\right) \delta 7.41-7.28(\mathrm{~m}, 5 \mathrm{H}), 5.24-5.10(\mathrm{~m}, 2 \mathrm{H}), 5.04(\mathrm{~d}, J=8.3 \mathrm{~Hz}, 1 \mathrm{H})$, $4.30(\mathrm{t}, J=7.1 \mathrm{~Hz}, 1 \mathrm{H}), 1.93-1.80(\mathrm{~m}, 1 \mathrm{H}), 1.69(\mathrm{dt}, J=14.2,7.2 \mathrm{~Hz}, 1 \mathrm{H}), 1.44(\mathrm{~s}, 9 \mathrm{H}), 0.90(\mathrm{t}$, $J=7.5 \mathrm{~Hz}, 3 \mathrm{H})$.

${ }^{13} \mathrm{C} \mathrm{NMR}\left(101 \mathrm{MHz}, \mathrm{CDCl}_{3}\right) \delta 172.8,155.5,135.6,128.7,128.5,128.4,79.9,67.1,54.8,28.5$, 26.1, 9.7.

Matching reported literature data. ${ }^{1}$<smiles>CN1CCCCC1</smiles>

1-tosylpiperidine (6b): Synthesized according to General Procedure F using 1tosylpiperidine-4-carboxylic acid (57 mg, $0.2 \mathrm{mmol}, 1$ equiv.). The crude mixture was purified by flash column chromatography on silica gel (10\% AcOEt in hexanes as eluent) to afford $\mathbf{6 b}$ (37 $\mathrm{mg}, 77 \%$ yield) as a white solid.

${ }^{1} \mathrm{H}$ NMR $\left(500 \mathrm{MHz}, \mathrm{CDCl}_{3}\right) \delta 7.66-7.60(\mathrm{~m}, 2 \mathrm{H}), 7.34-7.28(\mathrm{~m}, 2 \mathrm{H}), 2.99-2.93(\mathrm{~m}, 4 \mathrm{H})$, $2.42(\mathrm{~s}, 3 \mathrm{H}), 1.63(\mathrm{p}, J=5.9 \mathrm{~Hz}, 4 \mathrm{H}), 1.40(\mathrm{tt}, J=8.2,4.7 \mathrm{~Hz}, 2 \mathrm{H})$.

${ }^{13} \mathrm{C} \mathrm{NMR}\left(126 \mathrm{MHz}, \mathrm{CDCl}_{3}\right) \delta 143.4,133.5,129.7,127.8,47.1,25.3,23.7,21.6$.

Matching reported literature data. ${ }^{1}$ 
<smiles>CC(C)Oc1ccc(CCNC(=O)c2ccc(Cl)cc2)cc1</smiles>

4-chloro-N-(4-isopropoxyphenethyl)benzamide

(6c):

Synthesized according to General Procedure F using Bezafibrate (72 mg, $0.2 \mathrm{mmol}, 1$ equiv.). The crude mixture was purified by flash column chromatography on silica gel (25\% AcOEt in hexanes as eluent) to afford $\mathbf{6 c}$ ( $32 \mathrm{mg}, 50 \%$ yield) as a white solid.

${ }^{1} \mathrm{H}$ NMR $\left(400 \mathrm{MHz}, \mathrm{CDCl}_{3}\right)$ mixture of rotamers: $\delta 7.65-7.59(\mathrm{~m}, 2 \mathrm{H}), 7.37(\mathrm{dd}, J=8.6,2.0$ $\mathrm{Hz}, 2 \mathrm{H}), 7.15-7.04(\mathrm{~m}, 2 \mathrm{H}), 6.89-6.79(\mathrm{~m}, 2 \mathrm{H}), 6.13(\mathrm{~s}, 1 \mathrm{H}), 4.52(\mathrm{p}, J=6.1 \mathrm{~Hz}, 1 \mathrm{H}), 3.67$ (qd, $J=6.7,6.3,3.7 \mathrm{~Hz}, 2 \mathrm{H}), 2.85(\mathrm{t}, J=6.9 \mathrm{~Hz}, 2 \mathrm{H}), 1.33(\mathrm{~d}, J=6.1 \mathrm{~Hz}, 6 \mathrm{H})$.

${ }^{13} \mathrm{C} \mathrm{NMR}\left(101 \mathrm{MHz}, \mathrm{CDCl}_{3}\right)$ mixture of rotamers: $\delta 166.6,156.8,137.8,133.1,130.6,130.0$, $129.9,129.0,128.4,116.3,115.8,70.1,41.5,34.8,22.2$.

HRMS: calculated for $\mathrm{C}_{16} \mathrm{H}_{18} \mathrm{ClN}_{4} \mathrm{NaO}\left(\mathrm{M}+\mathrm{Na}^{+}\right)$: 340.1061 , found $340.1075(+4.1 \mathrm{ppm})$.

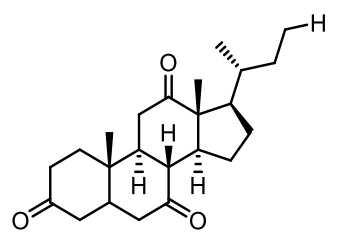

$(5 S, 8 R, 9 S, 10 S, 13 R, 14 S, 17 R)-17-($ sec-butyl)-10,13dimethyldodecahydro-3H-cyclopenta[a]phenanthrene3,7,12(2H,4H)-trione (6d): Synthesized according to General Procedure $\mathrm{F}$ using dehydrocholic acid $(81 \mathrm{mg}, 0.2 \mathrm{mmol}, 1$ equiv.). The crude mixture was purified by flash column chromatography on silica gel $(30 \%$ AcOEt in hexanes as eluent) to afford $\mathbf{6 d}$ (37 $\mathrm{mg}, 51 \%$ yield) as a light-

yellow solid.

${ }^{1} \mathrm{H} \mathrm{NMR}\left(400 \mathrm{MHz}, \mathrm{CDCl}_{3}\right) \delta 2.96-2.80(\mathrm{~m}, 3 \mathrm{H}), 2.39-2.17(\mathrm{~m}, 6 \mathrm{H}), 2.13(\mathrm{dd}, J=12.5,4.8$ $\mathrm{Hz}, 2 \mathrm{H}), 2.09-1.90(\mathrm{~m}, 4 \mathrm{H}), 1.85(\mathrm{td}, J=11.2,7.0 \mathrm{~Hz}, 1 \mathrm{H}), 1.68-1.56(\mathrm{~m}, 2 \mathrm{H}), 1.52-1.44$ $(\mathrm{m}, 1 \mathrm{H}), 1.40(\mathrm{~s}, 3 \mathrm{H}), 1.35-1.20(\mathrm{~m}, 3 \mathrm{H}), 1.20-1.10(\mathrm{~m}, 1 \mathrm{H}), 1.07(\mathrm{~s}, 3 \mathrm{H}), 0.85$ (dd, $J=15.9$, $6.9 \mathrm{~Hz}, 6 \mathrm{H})$.

${ }^{13} \mathrm{C} \mathrm{NMR}\left(101 \mathrm{MHz}, \mathrm{CDCl}_{3}\right) \delta 212.2,209.2,208.9,57.0,52.0,49.2,47.0,45.7,45.6,45.1,42.9$, $38.8,37.6,36.6,36.2,35.4,28.0,27.8,25.4,22.1,18.6,12.0,11.0$.

Matching reported literature data. ${ }^{1}$

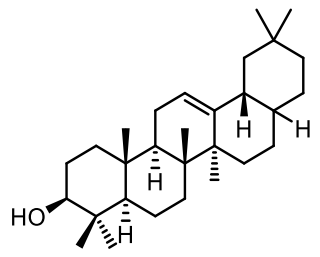

$(3 S, 4 \mathrm{a} R, 6 \mathrm{a} R, 6 \mathrm{~b} S, 12 \mathrm{a} R, 14 \mathrm{a} R, 14 \mathrm{~b} R)-4,4,6 \mathrm{a}, 6 \mathrm{~b}, 11,11,14 \mathrm{~b}-\mathrm{heptamethyl}-$

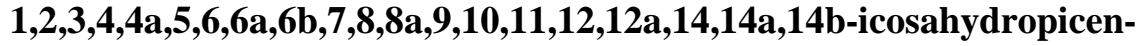
3-ol (6e): Synthesized according to General Procedure F using oleanolic acid (91 mg, $0.2 \mathrm{mmol}, 1$ equiv). The crude mixture was purified by flash column chromatography on silica gel (15\% AcOEt in hexanes as eluent) to afford $6 \mathbf{6}$ (74 $\mathrm{mg}, 90 \%$ yield) as white solid.

${ }^{1} \mathrm{H} \mathrm{NMR}\left(400 \mathrm{MHz}, \mathrm{CDCl}_{3}\right) \delta 5.19(\mathrm{t}, J=3.7 \mathrm{~Hz}, 1 \mathrm{H}), 3.22(\mathrm{ddz}, J=11.0,5.0 \mathrm{~Hz}, 1 \mathrm{H}), 2.34(\mathrm{dt}$, $J=13.7,4.8 \mathrm{~Hz}, 1 \mathrm{H}), 1.91-1.83(\mathrm{~m}, 2 \mathrm{H}), 1.83-1.75(\mathrm{~m}, 1 \mathrm{H}), 1.74-1.65(\mathrm{~m}, 2 \mathrm{H}), 1.65-1.58$ $(\mathrm{m}, 4 \mathrm{H}), 1.5-1.54(\mathrm{~m}, 2 \mathrm{H}), 1.50-1.31(\mathrm{~m}, 6 \mathrm{H}), 1.29-1.17(\mathrm{~m}, 4 \mathrm{H}), 1.11(\mathrm{~d}, J=0.9 \mathrm{~Hz}, 3 \mathrm{H})$, $1.08-1.04(\mathrm{~m}, 1 \mathrm{H}), 1.02-0.96(\mathrm{~m}, 4 \mathrm{H}), 0.93(\mathrm{~s}, 3 \mathrm{H}), 0.89(\mathrm{~s}, 3 \mathrm{H}), 0.87(\mathrm{~s}, 6 \mathrm{H}), 0.79(\mathrm{~s}, 3 \mathrm{H})$, $0.77-0.70(\mathrm{~m}, 1 \mathrm{H})$.

${ }^{13} \mathrm{C} \mathrm{NMR}\left(101 \mathrm{MHz}, \mathrm{CDCl}_{3}\right) \delta 146.1,121.2,79.2,55.4,47.9,45.1,42.6,41.1,39.3,38.9,38.6$, $37.3,35.9,33.8,33.8,33.2,31.3,31.2,29.9,28.3,28.1,27.4,25.2$, 24.0, 23.5, 22.4, 18.6, 17.6, 15.8, 15.5.

Matching reported literature data. ${ }^{1}$

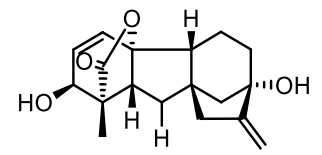

$(1 S, 2 S, 4 \mathrm{a} R, 4 \mathrm{~b} R, 7 S, 9 \mathrm{a} R, 10 \mathrm{a} R)-2,7-d i h y d r o x y-1-m e t h y l-8$-methylene-

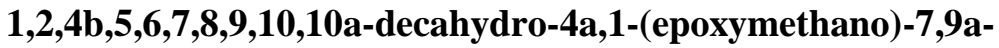
methanobenzo[a]azulen-13-one (6f): Synthesized according to General Procedure $\mathrm{F}$ using gibberellic acid ( $69 \mathrm{mg}, 0.2 \mathrm{mmol}, 1$ equiv.). The crude mixture was purified by flash column chromatography on silica gel (40\% AcOEt in hexanes as eluent) to afford $\mathbf{6 f}$ (36 $\mathrm{mg}, 60 \%$ yield) as a white solid. 
${ }^{1} \mathrm{H}$ NMR $\left(400 \mathrm{MHz}\right.$, Acetone- $\left.d_{6}\right) \delta 6.34(\mathrm{dd}, J=9.3,0.9 \mathrm{~Hz}, 1 \mathrm{H}), 5.85(\mathrm{dd}, J=9.3,3.7 \mathrm{~Hz}, 1 \mathrm{H})$, $5.16(\mathrm{td}, J=2.5,1.1 \mathrm{~Hz}, 1 \mathrm{H}), 4.81(\mathrm{tt}, J=2.1,1.0 \mathrm{~Hz}, 1 \mathrm{H}), 4.56-4.50(\mathrm{~m}, 1 \mathrm{H}), 4.01(\mathrm{dd}, J=6.4$, $3.6 \mathrm{~Hz}, 1 \mathrm{H}), 3.71(\mathrm{~s}, 1 \mathrm{H}), 2.85-2.74(\mathrm{~m}, 1 \mathrm{H}), 2.40(\mathrm{q}, J=2.0 \mathrm{~Hz}, 2 \mathrm{H}), 2.03-2.00(\mathrm{~m}, 1 \mathrm{H}), 1.91$ $(\mathrm{dd}, J=13.6,8.2 \mathrm{~Hz}, 1 \mathrm{H}), 1.87-1.80(\mathrm{~m}, 2 \mathrm{H}), 1.79-1.65(\mathrm{~m}, 3 \mathrm{H}), 1.61(\mathrm{dd}, J=13.6,11.0 \mathrm{~Hz}$, $1 \mathrm{H}), 1.53-1.47(\mathrm{~m}, 1 \mathrm{H}), 1.21(\mathrm{~s}, 3 \mathrm{H})$.

${ }^{13} \mathrm{C}$ NMR $\left(101 \mathrm{MHz}\right.$, Acetone- $\left.d_{6}\right) \delta 179.6,160.6,134.0,133.3,105.7,92.8,78.9,70.2,54.5,52.3$, 50.2, 48.2, 45.9, 40.1, 36.4, 30.2, 17.6, 15.2.

HRMS: calculated for $\mathrm{C}_{18} \mathrm{H}_{22} \mathrm{NaO}_{4}\left(\mathrm{M}+\mathrm{Na}^{+}\right)$: 325.1410, found 325.1400 (-3.3 ppm).

BocHN $\widehat{H}_{\mathrm{H}}$ tert-butyl (2-(4-chlorophenyl)propyl)carbamate (6g): Synthesized according to General Procedure F using 4-((tert-butoxycarbonyl)amino)-3-(4chlorophenyl)butanoic acid (63 mg, $0.2 \mathrm{mmol}, 1$ equiv.). The crude mixture was purified by flash column chromatography on silica gel (10\% AcOEt in hexanes as eluent) to afford $\mathbf{6 g}$ (40 $\mathrm{mg}, 74 \%$ yield) as colorless oil.

${ }^{1} \mathrm{H}$ NMR $\left(400 \mathrm{MHz}, \mathrm{CDCl}_{3}\right) \delta 7.34-7.20(\mathrm{~m}, 2 \mathrm{H}), 7.17-7.07(\mathrm{~m}, 2 \mathrm{H}), 4.41(\mathrm{~s}, 1 \mathrm{H}), 3.35(\mathrm{~s}$, $1 \mathrm{H}), 3.15(\mathrm{dd}, J=13.6,8.3 \mathrm{~Hz}, 1 \mathrm{H}), 2.91(\mathrm{q}, J=7.1 \mathrm{~Hz}, 1 \mathrm{H}), 1.41(\mathrm{~s}, 9 \mathrm{H}), 1.24(\mathrm{~d}, J=7.0 \mathrm{~Hz}$, $3 \mathrm{H})$.

${ }^{13} \mathrm{C} \mathrm{NMR}\left(101 \mathrm{MHz}, \mathrm{CDCl}_{3}\right) \delta 156.0,142.9,132.4,129.8,128.8,128.8,128.6,47.4,39.8,28.5$, 28.5, 19.2, 1.2.

Matching reported literature data. ${ }^{20}$

\section{C3.3 General Procedure G(deaminative reduction)}

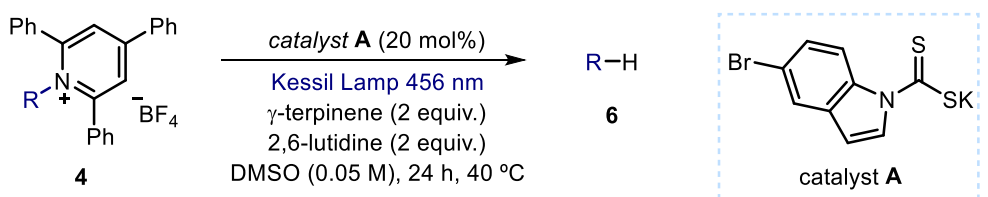

Reactions performed using set-up 2 in Figure S3. In an oven dried vial, with a Teflon septum screw cap, dithiocarbamate A (12.4 mg, $0.04 \mathrm{mmol}, 0.2$ equiv.) and pyridinium salt 4 (0.2 mmol, 1 equiv.) were dissolved in DMSO ( $4 \mathrm{~mL})$. Then, $\gamma$-terpinene $(64 \mu \mathrm{L}, 0.4 \mathrm{mmol}, 2$ equiv.) and 2,6-lutidine ( $46 \mu \mathrm{L}, 0.4 \mathrm{mmol}, 2$ equiv.) were added. The resulting orange mixture was degassed with argon sparging for 60 seconds. The vial was then placed at 2-3 cm of a $50 \mathrm{~W}$ Kessil blue LED lamp and irradiated under stirring for 16 hours (see Figure S3). The mixture was transferred to an extraction funnel, $\mathrm{NaHCO}_{3}$ sat. solution was added and the organic layer was extracted with EtOAc. The organic layer was washed with brine twice. The combined organic layers were dried over anhydrous $\mathrm{MgSO}_{4}$, filtered, and concentrated to dryness. The crude residue was purified by column chromatography to afford the corresponding product in the stated yield with $>95 \%$ purity according to ${ }^{1} \mathrm{H}$ NMR analysis.

\section{C3.4 Characterization of Products}

$\mathrm{Ph}$ 3-phenylpropan-1-ol (6h): Synthesized according to General Procedure G using 1(1-hydroxy-3-phenylpropan-2-yl)-2,4,6-triphenylpyridin-1-ium tetrafluoroborate $\mathbf{4 b}$ (106 mg, $0.2 \mathrm{mmol}, 1$ equiv.). The crude mixture was purified by flash column chromatography on silica gel (15\% AcOEt in pentane as eluent) to afford $\mathbf{6 h}$ ( $21 \mathrm{mg}, 77 \%$ yield) as a colorless oil.

${ }^{1} \mathrm{H} \mathrm{NMR}\left(400 \mathrm{MHz}, \mathrm{CDCl}_{3}\right) \delta 7.33-7.26(\mathrm{~m}, 2 \mathrm{H}), 7.23-7.17(\mathrm{~m}, 3 \mathrm{H}), 3.68(\mathrm{t}, J=6.4 \mathrm{~Hz}, 2 \mathrm{H})$, $2.72(\mathrm{dd}, J=8.7,6.8 \mathrm{~Hz}, 2 \mathrm{H}), 1.96-1.85(\mathrm{~m}, 2 \mathrm{H})$.

${ }^{13} \mathrm{C} \mathrm{NMR}\left(101 \mathrm{MHz}, \mathrm{CDCl}_{3}\right) \delta 141.9,128.6,128.5,126.0,62.4,34.4,32.2$.

Matching reported literature data. ${ }^{21}$ 
$\mathrm{C}_{\mathrm{H}}^{\mathrm{CO}_{2} \mathrm{Me}}$

methyl 3-(4-hydroxyphenyl)propanoate (6i): Synthesized according to General Procedure G using 1-(3-(4-hydroxyphenyl)-1-methoxy-1-oxopropan2-yl)-2,4,6-triphenylpyridin-1-ium tetrafluoroborate (115 mg, $0.2 \mathrm{mmol}, 1$ equiv.). The crude mixture was purified by flash column chromatography on silica gel $(0-40 \%$ AcOEt in hexanes as eluent) to afford $\mathbf{6 i}(28 \mathrm{mg}, 78 \%$ yield) as a colorless oil.

$\underline{{ }^{1} \mathrm{H} \mathrm{NMR}}\left(400 \mathrm{MHz}, \mathrm{CDCl}_{3}\right) \delta 7.04(\mathrm{~d}, J=8.4 \mathrm{~Hz}, 2 \mathrm{H}), 6.79-6.71(\mathrm{~m}, 2 \mathrm{H}), 5.74(\mathrm{~s}, 1 \mathrm{H}), 3.67$ (s, 3H), 2.88 (t, $J=7.7 \mathrm{~Hz}, 2 \mathrm{H}), 2.61$ (dd, $J=9.0,6.5 \mathrm{~Hz}, 2 \mathrm{H})$.

$\underline{{ }^{13} \mathrm{C} \mathrm{NMR}}\left(101 \mathrm{MHz}, \mathrm{CDCl}_{3}\right) \delta 174.1,154.4,132.4,129.5,115.5,51.9,36.2,30.2$.

Matching reported literature data. ${ }^{22}$

\section{C4. $\alpha$-Alkylation of Silyl Enol Ethers}

C4.1 General Procedure $H$

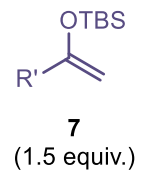

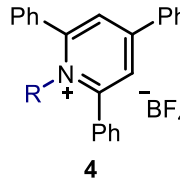
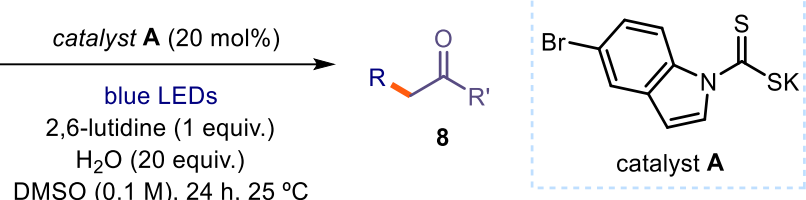

Reactions performed using set-up 3 in Figure S6. In an oven dried vial with a Teflon septum screw cap, silyl enol ether 7 ( $0.3 \mathrm{mmol}, 1.5$ equiv.) was dissolved in DMSO ( $2 \mathrm{~mL})$, followed by addition of 2,6-lutidine ( $23 \mu \mathrm{L}, 0.2 \mathrm{mmol}, 1.0$ equiv.), pyridinium salt 4 ( $0.2 \mathrm{mmol}, 1.0$ equiv.), catalyst A (12.4 mg, $0.04 \mathrm{mmol}, 0.2$ equiv.) and water ( $4.0 \mathrm{mmol}, 20$ equiv.). The resulting orange mixture was degassed by bubbling argon for 60 seconds. The vial was then placed in the irradiation setup (see Figure S6), maintained at a temperature of $25^{\circ} \mathrm{C}\left(25-26^{\circ} \mathrm{C}\right.$ measured in the central well), and the reaction was stirred for 24 hours under continuous irradiation from a blue LED strip, unless otherwise stated. The crude mixture was diluted with EtOAc and brine was added. The layers were separated, and the aqueous layer extracted with EtOAc $(\times 3)$. The combined organic fractions were dried over anhydrous $\mathrm{MgSO}_{4}$, filtered, and concentrated to dryness. The crude residue was purified by column chromatography on silica gel to afford the corresponding product in the stated yield with $>95 \%$ purity according to ${ }^{1} \mathrm{H}$ NMR analysis.

\section{C4.2 Characterization of Products with General Procedure H}

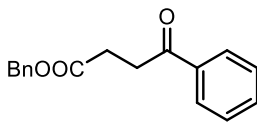

Benzyl 4-oxo-4-phenylbutanoate (8a): Prepared according to General Procedure H using set-up 2 (75\% intensity). 2,6-lutidine ( $46 \mu \mathrm{L}, 0.4 \mathrm{mmol}, 2$ equiv.), tert-butyldimethyl((1-phenylvinyl)oxy)silane 7a (70 mg, $0.4 \mathrm{mmol}, 2$ equiv.) and 1-(2-(benzyloxy)-2-oxoethyl)-2,4,6-triphenylpyridin-1-ium tetrafluoroborate 4d (109 mg, 0.2 mmol, 1 equiv.). Flash column chromatography (hexanes/EtOAc 95:5) to afford product $8 \mathbf{a}$ as an off-white oil (43 $\mathrm{mg}, 80 \%$ yield).

${ }^{1} \mathrm{H}$ NMR $\left(300 \mathrm{MHz}, \mathrm{CDCl}_{3}\right) \delta 8.03-7.94(\mathrm{~m}, 2 \mathrm{H}), 7.56(\mathrm{~m}, 1 \mathrm{H}), 7.46(\mathrm{~m}, 2 \mathrm{H}), 7.40-7.23(\mathrm{~m}$, $5 \mathrm{H}), 5.15(\mathrm{~s}, 3 \mathrm{H}), 3.34(\mathrm{t}, J=6.6 \mathrm{~Hz}, 2 \mathrm{H}), 2.83(\mathrm{t}, J=6.6 \mathrm{~Hz}, 2 \mathrm{H})$.

${ }^{13} \mathrm{C} \mathrm{NMR}\left(75 \mathrm{MHz}, \mathrm{CDCl}_{3}\right) \delta 198.2,172.9,136.7,136.0,133.4,128.8,128.7,128.4,128.2$,

66.7, 33.5, 28.43.

Matching reported literature data. ${ }^{23}$<smiles>O=CCCc1ccc(C(F)(F)F)cc1</smiles>

1-phenyl-3-(4-(trifluoromethyl)phenyl)propan-1-one (8b): Prepared according to General Procedure $\mathrm{H}$ using tert-butyldimethyl((1phenylvinyl)oxy)silane 7a (70 $\mathrm{mg}, 0.3 \mathrm{mmol}$ ) and 2,4,6-triphenyl-1-(4(trifluoromethyl)benzyl)pyridin-1-ium tetrafluoroborate $4 \mathbf{e}(111 \mathrm{mg}, 0.2$ 
mmol). Time of irradiation: 24 hours at $25^{\circ} \mathrm{C}$. Flash column chromatography (Toluene) to afford product $\mathbf{8 b}$ as a white solid ( $35 \mathrm{mg}, 55 \%$ yield).

${ }^{1} \mathrm{H} \mathrm{NMR}\left(300 \mathrm{MHz}, \mathrm{CDCl}_{3}\right) \delta 7.97-7.90(\mathrm{~m}, 2 \mathrm{H}), 7.59-7.50(\mathrm{~m}, 3 \mathrm{H}), 7.48-7.44(\mathrm{~m}, 2 \mathrm{H})$, $7.41-7.35(\mathrm{~m}, 2 \mathrm{H}), 3.33(\mathrm{t}, J=7.5 \mathrm{~Hz}, 2 \mathrm{H}), 3.15(\mathrm{t}, J=7.5 \mathrm{~Hz}, 2 \mathrm{H})$.

${ }^{13} \mathrm{C} \mathrm{NMR}\left(75 \mathrm{MHz}, \mathrm{CDCl}_{3}\right) \delta 198.2,145.5,136.8,136.8,133.4,129.1,128.8,128.7,128.1$, $125.4(\mathrm{q}, J=3.9 \mathrm{~Hz}), 124.5(\mathrm{q}, J=270 \mathrm{~Hz}), 39.9,29.9$.

${ }^{19} \mathrm{~F} \mathrm{NMR}\left(376 \mathrm{MHz}, \mathrm{CDCl}_{3}\right) \delta-62.49$.

Matching reported literature data. ${ }^{24}$<smiles>CNCCCCC(Cc1ccccc1)C(=O)OC</smiles>
Methyl 6-(((benzyloxy)carbonyl)amino)-2-(2-oxo-2-
phenylethyl)hexanoate (8c): Prepared according to General
Procedure H using tert-butyldimethyl((1-phenylvinyl)oxy)silane 7a
$(70 \mathrm{mg}, 0.3 \mathrm{mmol})$ and $( \pm)-1-(6-((($ benzyloxy $)$ carbonyl $)$ amino $)-1-$ methoxy-1-oxohexan-2-yl)-2,4,6-triphenylpyridin-1-ium tetrafluoroborate $\mathbf{4 f}$ (135 $\mathrm{mg}, 0.2$ mmol). Flash column chromatography (hexanes/EtOAc 7:3 to 1:1) to afford product $\mathbf{3 e}$ as a yellowish oil ( $53 \mathrm{mg}, 67 \%$ yield).

${ }^{1} \mathrm{H} \mathrm{NMR}\left(400 \mathrm{MHz}, \mathrm{CDCl}_{3}\right) \delta 7.99-7.93(\mathrm{~m}, 2 \mathrm{H}), 7.60-7.54(\mathrm{~m}, 1 \mathrm{H}), 7.49-7.44(\mathrm{~m}, 2 \mathrm{H})$, $7.36-7.30(\mathrm{~m}, 5 \mathrm{H}), 5.09(\mathrm{~s}, 2 \mathrm{H}), 4.76(\mathrm{~s}, 1 \mathrm{H}), 3.69(\mathrm{~s}, 3 \mathrm{H}), 3.56-3.34(\mathrm{~m}, 1 \mathrm{H}), 3.20(\mathrm{q}, J=$ $6.7 \mathrm{~Hz}, 2 \mathrm{H}), 3.15-2.99(\mathrm{~m}, 2 \mathrm{H}), 1.77-1.50(\mathrm{~m}, 5 \mathrm{H}), 1.39(\mathrm{~m}, 1 \mathrm{H})$.

${ }^{13} \mathrm{C} \mathrm{NMR}\left(101 \mathrm{MHz}, \mathrm{CDCl}_{3}\right) \delta 198.2,176.1,156.5,136.8,136.7,133.4,128.8,128.7,128.3$, 128.2, 66.8, 52.0, 40.9, 40.6, 40.3, 31.9, 29.9, 24.5.

HRMS: calculated for $\mathrm{C}_{23} \mathrm{H}_{27} \mathrm{NNaO}_{5}\left(\mathrm{M}+\mathrm{Na}^{+}\right)$: 420.1781, found 420.1771 (+2.3 ppm).

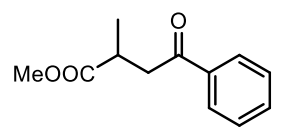

Benzyl 2-methyl-4-oxo-4-phenylbutanoate (8d): Prepared according to General Procedure H using tert-butyldimethyl((1-phenylvinyl)oxy)silane 7a (70 $\mathrm{mg}, \quad 0.3 \mathrm{mmol})$ and ( \pm )-1-(1-(benzyloxy)-1-oxopropan-2-yl)-2,4,6triphenylpyridin-1-ium tetrafluoroborate $\mathbf{4 g}(111 \mathrm{mg}, 0.2 \mathrm{mmol})$. Time of irradiation: 24 hours at $25^{\circ} \mathrm{C}$. Flash column chromatography (hexanes/EtOAc 98:2 to $90: 10$ ) to afford product 8d as a yellowish oil (39 mg, $69 \%$ yield).

${ }^{1} \mathrm{H} \mathrm{NMR}\left(300 \mathrm{MHz}, \mathrm{CDCl}_{3}\right) \delta 8.07-7.88(\mathrm{~m}, 2 \mathrm{H}), 7.72-7.53(\mathrm{~m}, 1 \mathrm{H}), 7.53-7.42(\mathrm{~m}, 2 \mathrm{H})$, $7.35(\mathrm{~m}, 5 \mathrm{H}), 5.22-5.09$ (dd, $J=16.5 \mathrm{~Hz}, 12.4 \mathrm{~Hz}, 2 \mathrm{H}), 3.52$ (dd, $J=17.5 \mathrm{~Hz}, 7.8 \mathrm{~Hz}, 1 \mathrm{H}$ ), $3.22(\mathrm{~m}, 1 \mathrm{H}), 3.06(\mathrm{dd}, J=17.5 \mathrm{~Hz}, 5.5 \mathrm{~Hz}, 1 \mathrm{H}), 1.32(\mathrm{~d}, J=7.1 \mathrm{~Hz}, 3 \mathrm{H})$.

${ }^{13} \mathrm{C} \mathrm{NMR}\left(75 \mathrm{MHz}, \mathrm{CDCl}_{3}\right) \delta 198.1,175.9,136.8,136.2,133.3,128.7,128 ., 128.2,128.2$, 128.2, 66.5, 42.0, 35.2, 17.4.

Matching reported literature data. ${ }^{23}$<smiles>O=CCCc1ncccn1</smiles>

1-phenyl-3-(pyrimidin-2-yl)propan-1-one (8e): Prepared according to General Procedure $\mathrm{H}$ at $40^{\circ} \mathrm{C}$ using tert-butyldimethyl((1-phenylvinyl)oxy)silane $7 \mathbf{a}$ (70 $\mathrm{mg}, \quad 0.3 \mathrm{mmol}$ ) and 2,4,6-triphenyl-1-(pyrimidin-2-ylmethyl)pyridin-1-ium tetrafluoroborate $\mathbf{4 h}$ (98 $\mathrm{mg}, 0.2 \mathrm{mmol})$. Flash column chromatography (hexanes/EtOAc 7:3) to afford product $8 \mathbf{e}$ as a yellowish oil (32 $\mathrm{mg}, 75 \%$ yield).

$\underline{{ }^{1} \mathrm{H} \mathrm{NMR}}\left(300 \mathrm{MHz}, \mathrm{CDCl}_{3}\right) \delta 8.66(\mathrm{~d}, J=4.9 \mathrm{~Hz}, 2 \mathrm{H}), 8.25-7.88(\mathrm{~m}, 2 \mathrm{H}), 7.61-7.52(\mathrm{~m}$, $1 \mathrm{H}), 7.51-7.39(\mathrm{~m}, 2 \mathrm{H}), 7.13(\mathrm{t}, J=4.9 \mathrm{~Hz}, 1 \mathrm{H}), 3.66-3.55(\mathrm{~m}, 2 \mathrm{H}), 3.46(\mathrm{~m}, 2 \mathrm{H})$.

${ }^{13} \mathrm{C} \mathrm{NMR}\left(75 \mathrm{MHz}, \mathrm{CDCl}_{3}\right) \delta$ 199.0, 170., 157.1, 137.1, 133.2, 128.7, 128.3, 118.8, 36.2, 33.1.

HRMS: calculated for $\mathrm{C}_{13} \mathrm{H}_{13} \mathrm{~N}_{2} \mathrm{O}\left(\mathrm{M}+\mathrm{H}^{+}\right)$: 213.1022, found 213.1020 (+1.0 ppm). 
<smiles>CC(CC(=O)c1ccccc1)c1cccnc1</smiles>

1-phenyl-3-(pyridin-3-yl)butan-1-one (8f): Prepared according to General Procedure $\mathrm{H}$ at $60{ }^{\circ} \mathrm{C}$ using tert-butyldimethyl((1-phenylvinyl)oxy)silane 7a (70 $\mathrm{mg}, 0.3 \mathrm{mmol})$ and $( \pm)-2,4,6$-triphenyl-1-(1-(pyridin-3-yl)ethyl)pyridin-1-ium tetrafluoroborate $4 \mathbf{i}(100 \mathrm{mg}, 0.2 \mathrm{mmol})$. Flash column chromatography (hexanes/EtOAc 8:2) to afford product $\mathbf{8 f}$ as a yellowish oil (20 $\mathrm{mg}, 44 \%$ yield).

${ }^{1} \mathrm{H} \mathrm{NMR}\left(400 \mathrm{MHz}, \mathrm{CDCl}_{3}\right) \delta 8.52(\mathrm{~d}, J=4.9,1 \mathrm{H}), 8.04-7.91(\mathrm{~m}, 2 \mathrm{H}), 7.64(\mathrm{td}, J=7.7 \mathrm{~Hz}$, $1.9 \mathrm{~Hz}, 1 \mathrm{H}), 7.58-7.49(\mathrm{~m}, 1 \mathrm{H}), 7.48-7.37(\mathrm{~m}, 2 \mathrm{H}), 7.30(\mathrm{~d}, J=7.8 \mathrm{~Hz}, 1 \mathrm{H}), 7.13(\mathrm{~m}, 1 \mathrm{H})$, $3.82-3.60(\mathrm{~m}, 2 \mathrm{H}), 3.25(\mathrm{dd}, J=16.4 \mathrm{~Hz}, 5.6 \mathrm{~Hz}, 1 \mathrm{H}), 1.40(\mathrm{~d}, J=6.8 \mathrm{~Hz}, 3 \mathrm{H})$.

${ }^{13} \mathrm{C} \mathrm{NMR}\left(101 \mathrm{MHz}, \mathrm{CDCl}_{3}\right) \delta 199.3,164.9,148.7,137.3,133.1,128.6,128.3,123.0,121.7$, 45.0, 37.2, 21.2.

HRMS: calculated for $\mathrm{C}_{15} \mathrm{H}_{16} \mathrm{NO}\left(\mathrm{M}+\mathrm{H}^{+}\right)$: 226.1226, found $226.1222(+1.8 \mathrm{ppm})$.

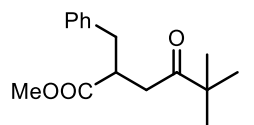

Methyl 2-benzyl-5,5-dimethyl-4-oxohexanoate (8g): Prepared according to General Procedure $\mathrm{H}$ with set-up 2 (75\% intensity) and solvent system DMSO/DCE (1:1). 2,6-Lutidine ( $47 \mu \mathrm{L}, 0.4 \mathrm{mmol}, 2.0$ equiv.), methyl 2benzyl-5,5-dimethyl-4-oxohexanoate $7 \mathbf{b}(119 \mathrm{mg}, 0.5 \mathrm{mmol})$ and ( \pm )-1-(1-methoxy-1-oxo-3phenylpropan-2-yl)-2,4,6-triphenylpyridin-1-ium tetrafluoroborate 41 (111 mg, $0.2 \mathrm{mmol}$ ). Flash column chromatography (Hexanes/EtOAc from 98:2 to 96:4) to afford product $8 \mathrm{~g}$ as a yellow oil (33 $\mathrm{mg}, 63 \%$ yield).

${ }^{1} \mathrm{H}$ NMR $\left(400 \mathrm{MHz}, \mathrm{CDCl}_{3}\right) \delta 7.31-7.26(\mathrm{~m}, 2 \mathrm{H}), 7.24-7.19(\mathrm{~m}, 1 \mathrm{H}), 7.17-7.10(\mathrm{~m}, 2 \mathrm{H})$, $3.63(\mathrm{~s}, 3 \mathrm{H}), 3.21-3.09(\mathrm{~m}, 1 \mathrm{H}), 3.01(\mathrm{dd}, J=13.6 \mathrm{~Hz}, 6.6 \mathrm{~Hz}, 1 \mathrm{H}), 2.93(\mathrm{dd}, J=18.1,8.9 \mathrm{~Hz}$, $1 \mathrm{H}), 2.73(\mathrm{dd}, J=13.6 \mathrm{~Hz}, 8.3 \mathrm{~Hz}, 1 \mathrm{H}), 2.52(\mathrm{dd}, J=18.1 \mathrm{~Hz}, 4.6 \mathrm{~Hz}, 1 \mathrm{H}), 1.10(\mathrm{~s}, 9 \mathrm{H})$.

${ }_{3}^{13} \mathrm{C} \mathrm{NMR}\left(101 \mathrm{MHz}, \mathrm{CDCl}_{3}\right) \delta 214.2,175.6,138.8,129.1,128.6,126.7,51.9,44.1,42.1,37.93$, 37.9, 26.5.

HRMS: calculated for $\mathrm{C}_{18} \mathrm{H}_{21} \mathrm{O}_{3}\left(\mathrm{M}+\mathrm{H}^{+}\right): 285.1485$, found 285.1475 (+3.5 ppm).<smiles>CC(=O)CC(Cc1ccccc1)C(=O)c1ccccc1</smiles>

Methyl 2-benzyl-4-oxo-4-phenylbutanoate (8h): Prepared according to General Procedure H using tert-butyldimethyl((1-phenylvinyl)oxy)silane 7a (70 mg, $0.3 \mathrm{mmol}$ ) and ( \pm )-1-(1-methoxy-1-oxo-3-phenylpropan-2-yl)-2,4,6triphenylpyridin-1-ium tetrafluoroborate $4 \mathbf{l}(111 \mathrm{mg}, 0.2 \mathrm{mmol})$. Flash column chromatography (hexanes/EtOAc 92:8) to afford product 3a as a yellowish oil (50 $\mathrm{mg}$, $89 \%$ yield).

${ }^{1} \underline{\mathrm{H} \mathrm{NMR}}\left(400 \mathrm{MHz}, \mathrm{CDCl}_{3}\right) \delta 7.93-7.87(\mathrm{~m}, 2 \mathrm{H}), 7.59-7.50(\mathrm{~m}, 1 \mathrm{H}), 7.43(\mathrm{dd}, J=8.4 \mathrm{~Hz}, 7.0$ $\mathrm{Hz}, 2 \mathrm{H}), 7.34-7.16(\mathrm{~m}, 5 \mathrm{H}), 3.67(\mathrm{~s}, 3 \mathrm{H}), 3.47-3.32(\mathrm{~m}, 2 \mathrm{H}), 3.12(\mathrm{dd}, J=13.6 \mathrm{~Hz}, 6.0 \mathrm{~Hz}$, $1 \mathrm{H}), 3.05-2.99(\mathrm{~m}, 1 \mathrm{H}), 2.85(\mathrm{dd}, J=13.6,8.1 \mathrm{~Hz}, 1 \mathrm{H})$.

${ }^{13} \mathrm{C} \mathrm{NMR}\left(101 \mathrm{MHz}, \mathrm{CDCl}_{3}\right) \delta 198.2,175.4,133.4,129.2,128.7,128.7,128.2,126.8,52.1,42.4$, 39.5, 38.0.

HRMS: calculated for $\mathrm{C}_{18} \mathrm{H}_{18} \mathrm{NaO}_{3}\left(\mathrm{M}+\mathrm{Na}^{+}\right)$: 305.1148, found 305.1142 (+2.0 ppm).

${ }^{\mathrm{Ph}} \mathrm{O} \quad$ Methyl 2-benzyl-4-oxo-4-(thiazol-2-yl)butanoate (8i): Prepared according to General Procedure $\mathrm{H}$ using 2-(1-((tertbutyldimethylsilyl)oxy)vinyl)thiazole $7 \mathbf{c}(73 \mathrm{mg}, 0.3 \mathrm{mmol})$ and $( \pm)-1-(1-$ methoxy-1-oxo-3-phenylpropan-2-yl)-2,4,6-triphenylpyridin-1-ium tetrafluoroborate $\mathbf{4 l}$ (111 mg, $0.2 \mathrm{mmol})$. Flash column chromatography (Hexanes/Acetone 95:5) to afford product $\mathbf{8 i}$ as a yellowish solid (46 $\mathrm{mg}, 79 \%$ yield). 
${ }^{1} \mathrm{H} \mathrm{NMR}\left(300 \mathrm{MHz}, \mathrm{CDCl}_{3}\right) \delta 7.98(\mathrm{~d}, J=3.0 \mathrm{~Hz}, 1 \mathrm{H}), 7.65(\mathrm{~d}, J=3.0 \mathrm{~Hz}, 1 \mathrm{H}), 7.33-7.26(\mathrm{~m}$, $2 \mathrm{H}), 7.23-7.18(\mathrm{~m}, 3 \mathrm{H}), 3.76-3.58(\mathrm{~m}, 4 \mathrm{H}), 3.40-3.30(\mathrm{~m}, 1 \mathrm{H}), 3.26-3.10(\mathrm{~m}, 2 \mathrm{H}), 2.84$ (dd, $J=13.6,8.6 \mathrm{~Hz}, 1 \mathrm{H})$.

${ }^{13} \mathrm{C} \mathrm{NMR}\left(75 \mathrm{MHz}, \mathrm{CDCl}_{3}\right) \delta 192.0(\mathrm{C}), 174.9(\mathrm{C}), 166.7(\mathrm{C}), 144.9(\mathrm{CH}), 138.5(\mathrm{C}), 129.2$ $(\mathrm{CH}), 128.7(\mathrm{CH}), 126.8(\mathrm{CH}), 126.4(\mathrm{CH}), 52.1\left(\mathrm{CH}_{3}\right), 42.3(\mathrm{CH}), 39.5\left(\mathrm{CH}_{2}\right), 38.0\left(\mathrm{CH}_{2}\right)$.

HRMS: calculated for $\mathrm{C}_{15} \mathrm{H}_{15} \mathrm{NNaO}_{3} \mathrm{~S}\left(\mathrm{M}+\mathrm{Na}^{+}\right): 312.0665$, found 312.0665 (+0.0 ppm).<smiles>COC(CC(=O)c1cc(Br)cc2c1CCC2(C)C)Cc1ccccc1</smiles>

Methyl 2-benzyl-4-(6-(tert-butyl)-1,1-dimethyl-2,3-dihydro-1Hinden-4-yl)-4-oxobutanoate (8j): Prepared according to General Procedure $\mathrm{H}$ using tert-butyl((1-(6-(tert-butyl)-1,1-dimethyl-2,3dihydro-1H-inden-4-yl)vinyl)oxy)dimethylsilane $7 \mathbf{d}$ (108 mg, 0.3 $\mathrm{mmol})$ and ( \pm )-1-(1-methoxy-1-oxo-3-phenylpropan-2-yl)-2,4,6triphenylpyridin-1-ium tetrafluoroborate $4 \mathbf{l}(111 \mathrm{mg}, 0.2 \mathrm{mmol})$. Flash column chromatography (Hexanes/EtOAc 98:2) to afford product $\mathbf{8 j}$ as a yellowish oil (74 $\mathrm{mg}, 91 \%$ yield).

${ }^{1} \mathrm{H} \mathrm{NMR}\left(400 \mathrm{MHz}, \mathrm{CDCl}_{3}\right) \delta 7.62(\mathrm{~d}, J=1.8 \mathrm{~Hz}, 1 \mathrm{H}), 7.33-7.26(\mathrm{~m}, 3 \mathrm{H}), 7.24-7.17(\mathrm{~m}, 3 \mathrm{H})$, $3.66(\mathrm{~s}, 3 \mathrm{H}), 3.47-3.22(\mathrm{~m}, 2 \mathrm{H}), 3.14-3.07(\mathrm{~m}, 3 \mathrm{H}), 3.05-2.97(\mathrm{~m}, 1 \mathrm{H}), 2.84(\mathrm{dd}, J=13.5$, $7.9 \mathrm{~Hz}, 1 \mathrm{H}), 1.94-1.87(\mathrm{~m}, 2 \mathrm{H}), 1.33(\mathrm{~s}, 9 \mathrm{H}), 1.24(\mathrm{~d}, J=2.6 \mathrm{~Hz}, 6 \mathrm{H})$.

${ }^{13} \mathrm{C}$ NMR $\left(101 \mathrm{MHz}, \mathrm{CDCl}_{3}\right) \delta 200.2,175.6,154.5,150.1,141.2,138.9,133.3,129.2,128.6$, $126.7,124.0,123.5,52.0,43.6,42.6,41.6,41.5,38.1,34.9,31.6,30.88,28.9$.

HRMS: calculated for $\mathrm{C}_{27} \mathrm{H}_{34} \mathrm{NaO}_{3}\left(\mathrm{M}+\mathrm{Na}^{+}\right)$: 429.2400, found 429.2409 (+2.1 ppm).<smiles>COc1ccc(C(=O)CC(Cc2ccccc2)C(C)=O)cc1</smiles>

Methyl 2-benzyl-4-(4-methoxyphenyl)-4-oxobutanoate (8k): Prepared according to General Procedure $\mathrm{H}$ using tert-butyl((1-(4methoxyphenyl)vinyl)oxy)dimethylsilane 7 e ( $80 \mathrm{mg}, 0.3 \mathrm{mmol})$ and $( \pm)$ 1-(1-methoxy-1-oxo-3-phenylpropan-2-yl)-2,4,6-triphenylpyridin-1-ium tetrafluoroborate $4 \mathbf{l}$ (111 mg, $0.2 \mathrm{mmol})$. Flash column chromatography (Hexanes/EtOAc 93:7) to afford product $\mathbf{8 k}$ as an off-white solid (52 $\mathrm{mg}, 83 \%$ yield).

${ }^{1} \mathrm{H} \mathrm{NMR}\left(400 \mathrm{MHz}, \mathrm{CDCl}_{3}\right) \delta 7.88(\mathrm{~d}, J=9.0 \mathrm{~Hz}, 2 \mathrm{H}), 7.32-7.27(\mathrm{~m}, 2 \mathrm{H}), 7.24-7.17(\mathrm{~m}$, $3 \mathrm{H}), 6.90(\mathrm{~d}, J=9.0 \mathrm{~Hz}, 2 \mathrm{H}), 3.86(\mathrm{~s}, 3 \mathrm{H}), 3.66(\mathrm{~s}, 3 \mathrm{H}), 3.44-3.26(\mathrm{~m}, 2 \mathrm{H}), 3.10(\mathrm{dd}, J=13.6$, $5.9 \mathrm{~Hz}, 1 \mathrm{H}), 2.97(\mathrm{~d}, J=12.9 \mathrm{~Hz}, 1 \mathrm{H}), 2.84(\mathrm{dd}, J=13.6,7.9 \mathrm{~Hz}, 1 \mathrm{H})$.

${ }^{13} \mathrm{C} \mathrm{NMR}\left(101 \mathrm{MHz}, \mathrm{CDCl}_{3}\right) \delta 196.7,175.6,163.7,138.7,130.4,129.9,129.2,128.7,126.8$, 113.8, 55.6, 52.0, 42.4, 39.2, 38.0.

HRMS: calculated for $\mathrm{C}_{27} \mathrm{H}_{34} \mathrm{NaO}_{3}\left(\mathrm{M}+\mathrm{Na}^{+}\right)$: 429.2400, found 429.2409 (-2.1 ppm).

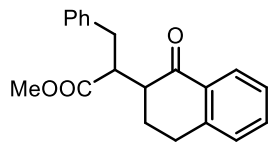

Methyl 2-benzyl-4-(6-(tert-butyl)-1,1-dimethyl-2,3-dihydro-1H-inden-4yl)-4-oxobutanoate (81): Prepared according to General Procedure $\mathrm{H}$ using tert-butyl((3,4-dihydronaphthalen-1-yl)oxy)dimethylsilane 7f $(104 \mathrm{mg}, 0.4$ $\mathrm{mmol})$ and $( \pm)-1-(1-m e t h o x y-1-o x o-3-p h e n y l p r o p a n-2-y l)-2,4,6-$ triphenylpyridin-1-ium tetrafluoroborate 41 (111 mg, $0.2 \mathrm{mmol})$. Flash column chromatography (Hexanes/EtOAc from 95:5 to 85:15) to afford product $\mathbf{3 m}$ as an off-green solid (50 $\mathrm{mg}, 81 \%$ yield, $1: 1 \mathrm{dr}$ ).

${ }^{1} \mathrm{H}$ NMR $\left(300 \mathrm{MHz}, \mathrm{CDCl}_{3}\right)(1: 1$ mixture of diasteroisomers) $\delta 8.02$ (ddd, $J=9.7,7.8,1.5 \mathrm{~Hz}$, $1 \mathrm{H}), 7.54-7.39(\mathrm{~m}, 1 \mathrm{H}), 7.35-7.26(\mathrm{~m}, 3 \mathrm{H}), 7.26-7.14(\mathrm{~m}, 4 \mathrm{H}), 3.66-3.60(\mathrm{~m}, 0.4 \mathrm{H}), 3.59$ $(\mathrm{s}, 1.1 \mathrm{H}), 3.57(\mathrm{~s}, 1.8 \mathrm{H}), 3.47-3.38(\mathrm{~m}, 0.6 \mathrm{H}), 3.26-3.17(\mathrm{~m}, 0.5 \mathrm{H}), 3.05-2.75(\mathrm{~m}, 4 \mathrm{H})$, $2.66-2.58(\mathrm{~m}, 0.7 \mathrm{H}), 2.19-2.32(\mathrm{~m}, 1 \mathrm{H}), 1.94-1.87(\mathrm{~m}, 1 \mathrm{H})$. 
${ }^{13} \mathrm{C} \mathrm{NMR}\left(75 \mathrm{MHz}, \mathrm{CDCl}_{3}\right)(1: 1$ mixture of diasteroisomers) $\delta 197.8,175.3,174.3,143.9$, $143.7,139.9,139.3,133.7,133.5,132.6,129.2,129.0,128.8,128.8,128.7,128.5,127.7,127.7$, 126.9, 126.8, 126.6, 126.5, 51.80, 50.3, 48.7, 47.3, 46.1, 35.4, 34.7, 29.6, 29.2, 26.1, 25.4. HRMS: calculated for $\mathrm{C}_{20} \mathrm{H}_{20} \mathrm{NaO}_{3}\left(\mathrm{M}+\mathrm{Na}^{+}\right)$: 331.1305 , found $331.1289(+4.8 \mathrm{ppm})$.

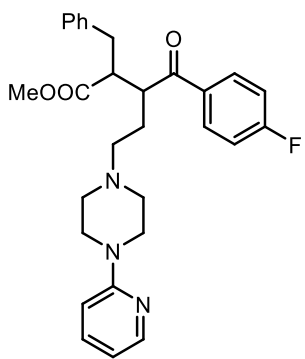

\begin{abstract}
Methyl 2-benzyl-3-(4-fluorobenzoyl)-5-(4-(pyridin-2-yl)piperazin-1yl)pentanoate (8m): Prepared according to General Procedure $\mathrm{H}$ using $(Z)$ 1-(4-((tert-butyldimethylsilyl)oxy)-4-(4-fluorophenyl)but-3-en-1-yl)-4(pyridin-2-yl)piperazine $\mathbf{7 g}(133 \mathrm{mg}, 0.3 \mathrm{mmol})$ and ( \pm )-1-(1-methoxy-1oxo-3-phenylpropan-2-yl)-2,4,6-triphenylpyridin-1-ium tetrafluoroborate $4 \mathrm{l}(111 \mathrm{mg}, 0.2 \mathrm{mmol})$, no water is added into the reaction mixture. Flash column chromatography (Hexanes/Acetone from 7:3 to 1:1) to afford product $\mathbf{8 m}$ as an off-white solid (49 $\mathrm{mg}, 50 \%$ yield, 1.2:1 dr).
\end{abstract}

${ }^{1} \mathrm{H} \mathrm{NMR}\left(500 \mathrm{MHz}, \mathrm{CDCl}_{3}\right) \delta 8.14(\mathrm{~m}, 1 \mathrm{H}), 8.05-7.97(\mathrm{~m}, 2 \mathrm{H}), 7.47-7.39(\mathrm{~m}, 1 \mathrm{H}), 7.25-$ $7.14(\mathrm{~m}, 4 \mathrm{H}), 7.13-7.04(\mathrm{~m}, 5 \mathrm{H}), 6.62-6.51(\mathrm{~m}, 2 \mathrm{H}), 3.95-3.82(\mathrm{~m}, 1 \mathrm{H}), 3.49(\mathrm{~s}, 1 \mathrm{H}), 3.44$ $(\mathrm{s}, 2 \mathrm{H}), 3.28(\mathrm{t}, J=44.1 \mathrm{~Hz}, 4 \mathrm{H}), 3.12-3.04(\mathrm{~m}, 1 \mathrm{H}), 2.80-2.61(\mathrm{~m}, 1 \mathrm{H}), 2.34(\mathrm{~d}, J=37.0$ $\mathrm{Hz}, 6 \mathrm{H}), 2.13$ (ddd, $J=13.9,10.4,6.4 \mathrm{~Hz}, 1 \mathrm{H}), 1.99$ (s, 1H), 1.70 (s, 1H).

${ }^{13} \mathrm{C} \mathrm{NMR}\left(126 \mathrm{MHz}, \mathrm{CDCl}_{3}\right)$ 1:1 mixture of diasteroisomer $\delta 200.5,200.1,174.5,174.4,159.5$, 148.1, 148.1, 138.8, 138.6, 137.6, 137.6 135.1, 134.0, 131.2, 131.2, 131.1, 131.0, 129.0, 128.9, $128.6,128.5,126.7,126.7,115.9,115.9,115.8,115.7,113.5,113.4,107.2,107.1,56.1,55.9$, $52.8,52.6,51.8,51.6,51.4,49.2,45.9,45.6,44.9,44.8,37.2,34.9$.

${ }^{19} \mathrm{~F} \mathrm{NMR}\left(376 \mathrm{MHz}, \mathrm{CDCl}_{3}\right)$ 1:1 mixture of diasteroisomer $\delta-105.4(\mathrm{~s}, 1 \mathrm{~F}),-105.6(\mathrm{~s}, 1 \mathrm{~F})$.

HRMS: calculated for $\mathrm{C}_{29} \mathrm{H}_{33} \mathrm{FN}_{3} \mathrm{O}_{3}\left(\mathrm{M}+\mathrm{H}^{+}\right)$: 490.2500, found $490.2500(0.0 \mathrm{ppm})$.<smiles>O=C(CC1CCCCC1)c1ccccc1</smiles>

Methyl 2-benzyl-5,5-dimethyl-4-oxohexanoate (8n): Prepared according to General Procedure H with set-up 1 and no 2,6-Lutidine. tert-butyldimethyl((1phenylvinyl)oxy)silane $7 \mathbf{a}(70 \mathrm{mg}, 0.3 \mathrm{mmol})$ and 1,3-dioxoisoindolin-2-yl cyclohexanecarboxylate 1a $(54.5 \mathrm{mg}, 0.2 \mathrm{mmol}, 1$ equiv.). Flash column chromatography (Hexanes/EtOAc from 100:0 to 98:2) to afford product 8n as a yellow oil (17 $\mathrm{mg}, 42 \%$ yield).

${ }^{1} \mathrm{H}$ NMR $\left(400 \mathrm{MHz}, \mathrm{CDCl}_{3}\right) \delta 7.98-7.92(\mathrm{~m}, 2 \mathrm{H}), 7.58-7.51(\mathrm{~m}, 1 \mathrm{H}), 7.49-7.42(\mathrm{~m}, 2 \mathrm{H})$, $2.82(\mathrm{~d}, J=6.8 \mathrm{~Hz}, 2 \mathrm{H}), 2.04-1.93(\mathrm{~m}, 1 \mathrm{H}), 1.80-1.73(\mathrm{~m}, 2 \mathrm{H}), 1.73-1.61(\mathrm{~m}, 2 \mathrm{H}), 1.34-$ $1.23(\mathrm{~m}, 3 \mathrm{H}), 1.22-1.14(\mathrm{~m}, 1 \mathrm{H}), 1.07-0.96(\mathrm{~m}, 2 \mathrm{H})$.

${ }^{13} \mathrm{C} \mathrm{NMR}\left(101 \mathrm{MHz}, \mathrm{CDCl}_{3}\right) \delta 200.5,137.6,133.0,128.7,128.3,46.4,34.7,33.6,26.4,26.3$.

Matching reported literature data. ${ }^{25}$<smiles>O=C(CCCCc1ccccc1)c1ccccc1</smiles>

Methyl 2-benzyl-5,5-dimethyl-4-oxohexanoate (80): Prepared according to General Procedure $\mathrm{H}$ with set-up 1 and no 2,6-Lutidine. tert-butyldimethyl((1-phenylvinyl)oxy)silane $7 \mathbf{a}(70 \mathrm{mg}, 0.3 \mathrm{mmol})$ and 1,3-dioxoisoindolin-2-yl 4-phenylbutanoate $\mathbf{1 b}(62 \mathrm{mg}, 0.2 \mathrm{mmol}, 1$ equiv.). Flash column chromatography (Hexanes/EtOAc from 100:0 to 98:2) to afford product 8o as a yellow oil (18 $\mathrm{mg}, 38 \%$ yield).

${ }^{1} \mathrm{H}$ NMR $\left(400 \mathrm{MHz}, \mathrm{CDCl}_{3}\right) \delta 7.97-7.92(\mathrm{~m}, 2 \mathrm{H}), 7.59-7.52(\mathrm{~m}, 1 \mathrm{H}), 7.49-7.42(\mathrm{~m}, 2 \mathrm{H})$, $7.31-7.21(\mathrm{~m}, 2 \mathrm{H}), 7.19(\mathrm{dt}, J=8.0,1.8 \mathrm{~Hz}, 3 \mathrm{H}), 2.99(\mathrm{t}, J=7.2 \mathrm{~Hz}, 2 \mathrm{H}), 2.67(\mathrm{t}, J=7.5 \mathrm{~Hz}$, $2 \mathrm{H}), 1.83-1.76(\mathrm{~m}, 2 \mathrm{H}), 1.76-1.68(\mathrm{~m}, 2 \mathrm{H})$. 
${ }^{13} \mathrm{C} \mathrm{NMR}\left(101 \mathrm{MHz}, \mathrm{CDCl}_{3}\right) \delta 200.4,142.4,137.2,133.1,128.7,128.5,128.5,128.2,125.9$,

38.5, 35.9, 31.2, 29.8, 24.1 .

Matching reported literature data. ${ }^{26}$

C4.3 General Procedure I (three-component reaction)

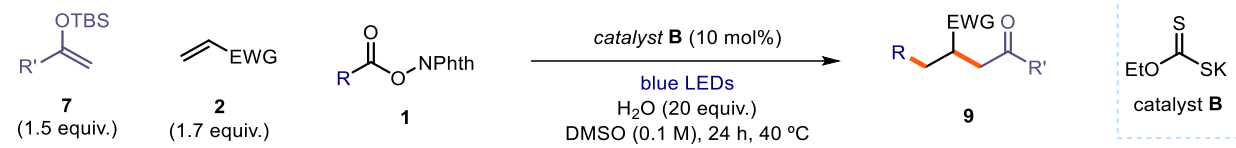

Reactions performed using set-up 1 in Figure S2. In an oven dried vial, with a Teflon septum screw cap, silyl enol ether 7 (0.3 mmol, 1.5 equiv.) was dissolved in DMSO (2 mL), then phthalimide ester 1 ( $0.2 \mathrm{mmol}, 1.0$ equiv. $)$ was added, followed by catalyst $\mathbf{B}(3.2 \mathrm{mg}, 0.02 \mathrm{mmol}$, 0.1 equiv.), electron-poor olefin 2 ( $0.34 \mathrm{mmol}, 1.7$ equiv.) and water ( $4.0 \mathrm{mmol}, 20$ equiv.). The resulting orange mixture was degassed by bubbling argon for 60 seconds. The vial was then placed in the 3D printed support photoreactor (Figure S2) and irradiated under stirring for 24 hours, unless otherwise specified. The crude mixture was diluted with EtOAc and brine was added. The combined organic fractions were dried over anhydrous $\mathrm{MgSO}_{4}$ and concentrated to dryness. The crude residue was purified by column chromatography on silica gel to afford the corresponding product in the stated yield with $>95 \%$ purity according to ${ }^{1} \mathrm{H}$ NMR analysis.

\section{C4.4 Characterization of Products}

CN O 2-(cyclohexylmethyl)-4-oxo-4-phenylbutanenitrile (9a): Prepared according to General Procedure I using tert-butyldimethyl((1-phenylvinyl)oxy)silane 7a (63 $\mathrm{mg}, 0.24 \mathrm{mmol}), 1,3$-dioxoisoindolin-2-yl cyclohexanecarboxylate $1 \mathrm{a}(55 \mathrm{mg}, 0.2 \mathrm{mmol})$, and acrylonitrile $\mathbf{2 b}(22 \mu \mathrm{L} 0.34 \mathrm{mmol})$. Flash column chromatography (hexanes/EtOAc 94:6) to afford product $9 \mathbf{a}$ as an off-white solid (31 $\mathrm{mg}, 61 \%$ yield).

${ }^{1} \mathrm{H}$ NMR $\left(300 \mathrm{MHz}, \mathrm{CDCl}_{3}\right) \delta 8.04-7.86(\mathrm{~m}, 2 \mathrm{H}), 7.72-7.59(\mathrm{~m}, 1 \mathrm{H}), 7.52-7.45(\mathrm{~m}, 2 \mathrm{H})$, $3.52-3.30(\mathrm{~m}, 2 \mathrm{H}), 3.30-3.13(\mathrm{~m}, 1 \mathrm{H}), 1.89(\mathrm{~d}, J=12.9 \mathrm{~Hz}, 1 \mathrm{H}), 1.83-1.57(\mathrm{~m}, 6 \mathrm{H}), 1.49-$ $1.40(\mathrm{~m}, 1 \mathrm{H}), 1.24-1.08(\mathrm{~m}, 2 \mathrm{H}), 1.06-0.83(\mathrm{~m}, 3 \mathrm{H})$.

${ }^{13} \mathrm{C} \mathrm{NMR}\left(75 \mathrm{MHz}, \mathrm{CDCl}_{3}\right) \delta 195.5,136.1,134.0,129.0,128.2,122.3,41.5,39.9,35.7,33.8$, 32.2, 26.5, 26.2, 26.0, 24.0.

HRMS: calculated for $\mathrm{C}_{17} \mathrm{H}_{21} \mathrm{NNaO}\left(\mathrm{M}+\mathrm{Na}^{+}\right)$: 278.1515, found 278.1511 (+1.4 ppm).<smiles></smiles>

mmol),

4-chloro-N-(4-((4-cyano-2-methyl-6-oxo-6phenylhexan-2-yl)oxy)phenethyl)benzamide $\quad(9 \mathrm{~b})$ : Prepared according to General Procedure I using tertbutyldimethyl((1-phenylvinyl)oxy)silane $7 \mathbf{a}(63 \mathrm{mg}, 0.24$ 2-(4-(2-(4-chlorobenzamido)ethyl)phenoxy)-2-

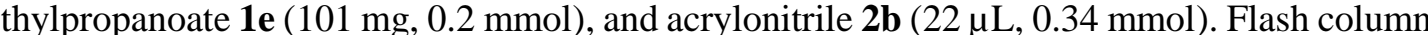
chromatography (hexanes/EtOAc from 7:3 to 1:1) to afford product $\mathbf{9 b}$ as a yellowish fluffy solid (51 mg, 52\% yield).

${ }^{1} \mathrm{H} \mathrm{NMR}\left(300 \mathrm{MHz}, \mathrm{CDCl}_{3}\right) \delta 8.05-7.86(\mathrm{~m}, 2 \mathrm{H}), 7.64(\mathrm{~d}, J=8.4 \mathrm{~Hz}, 3 \mathrm{H}), 7.57-7.46(\mathrm{~m}$, $2 \mathrm{H}), 7.42-7.38(\mathrm{~m}, 2 \mathrm{H}), 7.14(\mathrm{~d}, J=8.4 \mathrm{~Hz}, 2 \mathrm{H}), 6.98(\mathrm{~d}, J=8.4 \mathrm{~Hz}, 2 \mathrm{H}), 6.09(\mathrm{~s}, 1 \mathrm{H}), 3.74-$ $3.61(\mathrm{~m}, 3 \mathrm{H}), 3.57-3.37(\mathrm{~m}, 2 \mathrm{H}), 2.90(\mathrm{t}, J=6.9 \mathrm{~Hz}, 2 \mathrm{H}), 2.23(\mathrm{dd}, J=14.2,9.2 \mathrm{~Hz}, 1 \mathrm{H}), 1.99$ $(\mathrm{dd}, J=14.2,3.8 \mathrm{~Hz}, 1 \mathrm{H}), 1.44(\mathrm{~s}, 3 \mathrm{H}), 1.40(\mathrm{~s}, 3 \mathrm{H})$.

${ }^{13} \mathrm{C} \mathrm{NMR}\left(75 \mathrm{MHz}, \mathrm{CDCl}_{3}\right) \delta 195.8,166.5,153.4,136.1,134.2,134.0,133.5,129.6,129.0$, $128.4,128.2,124.3,79.2,44.8,42.3,35.0,27.1,26.2,21.9$.

HRMS: calculated for $\mathrm{C}_{29} \mathrm{H}_{29} \mathrm{ClN}_{2} \mathrm{NaO}_{3}\left(\mathrm{M}+\mathrm{Na}^{+}\right)$: 511.1759, found 511.1759 (+0.0 ppm). 
$\underbrace{-0}_{\text {COOMe }}$

Dimethyl 2-cyclohexyl-3-(2-oxo-2-phenylethyl)succinate (9c): Prepared according to General Procedure I using tert-butyldimethyl((1phenylvinyl)oxy)silane $\mathbf{7 a}(63 \mathrm{mg}, 0.24 \mathrm{mmol})$, 1,3-dioxoisoindolin-2-yl cyclohexanecarboxylate 1a $(55 \mathrm{mg}, 0.2 \mathrm{mmol})$, and dimethylfumarate $\mathbf{2 c}$ (43 $\mathrm{mg}$, $0.3 \mathrm{mmol}$ ). Flash column chromatography (hexanes/EtOAc 88:12) to afford the two separable diastereoisomeric products 9c (major diasteroisomer) and 9c' (minor diastereoisomer) as offwhite solids (d.r. 1:2.5, $50 \mathrm{mg}, 72 \%$ yield).

Spectroscopic data for $\mathbf{9 c}$ :

${ }^{1} \mathrm{H}$ NMR $\left(300 \mathrm{MHz}, \mathrm{CDCl}_{3}\right) \delta 8.05-7.86(\mathrm{~m}, 2 \mathrm{H}), 7.63-7.52(\mathrm{~m}, 1 \mathrm{H}), 7.52-7.45(\mathrm{~m}, 2 \mathrm{H})$, $3.69(\mathrm{~s}, 3 \mathrm{H}), 3.67(\mathrm{~s}, 3 \mathrm{H}), 3.67-3.59(\mathrm{~m}, 1 \mathrm{H}), 3.46-2.94(\mathrm{~m}, 2 \mathrm{H}), 2.71(\mathrm{dd}, J=8.7,5.6 \mathrm{~Hz}$, $1 \mathrm{H}), 1.81-1.59(\mathrm{~m}, 6 \mathrm{H}), 1.22-1.17(\mathrm{~m}, 2 \mathrm{H}), 1.09-0.77(\mathrm{~m}, 3 \mathrm{H})$.

${ }^{13} \mathrm{C} \mathrm{NMR}\left(75 \mathrm{MHz}, \mathrm{CDCl}_{3}\right) \delta 198.3,174.7,173.8,136.7,133.4,128.7,128.3,52.6,52.3,51.7$, $39.8,37.4,36.6,31.1,30.4,26.3,26.3,26.2$.

HRMS: calculated for $\mathrm{C}_{20} \mathrm{H}_{26} \mathrm{NaO}_{5}\left(\mathrm{M}+\mathrm{Na}^{+}\right)$: 369.1677, found 369.1672 (+1.4 ppm).

Spectroscopic data for $\mathbf{9}$ ':

${ }^{1} \mathrm{H}$ NMR $\left(300 \mathrm{MHz}, \mathrm{CDCl}_{3}\right) \delta 8.01-7.94(\mathrm{~m}, 2 \mathrm{H}), 7.61-7.54(\mathrm{~m}, 1 \mathrm{H}), 7.50-7.42(\mathrm{~m}, 2 \mathrm{H})$, $3.68(\mathrm{~s}, 3 \mathrm{H}), 3.68(\mathrm{~s}, 3 \mathrm{H}), 3.58-3.47(\mathrm{~m}, 2 \mathrm{H}), 3.24-3.10(\mathrm{~m}, 1 \mathrm{H}), 2.66-2.55(\mathrm{~m}, 1 \mathrm{H}), 1.96$ $(\mathrm{m}, 1 \mathrm{H}), 1.80-1.64(\mathrm{~m}, 6 \mathrm{H}), 1.07-0.85(\mathrm{~m}, 5 \mathrm{H})$.

${ }^{13} \mathrm{C} \mathrm{NMR}\left(75 \mathrm{MHz}, \mathrm{CDCl}_{3}\right) \delta 197.9,174.2,173.9,136.8,133.4,128.8,128.2,53.4,52.2,51.7$, 39.3, 38.1, 37.4, 31.1, 30.9, 26.4, 26.4.

HRMS: calculated for $\mathrm{C}_{20} \mathrm{H}_{26} \mathrm{NaO}_{5}\left(\mathrm{M}+\mathrm{Na}^{+}\right)$: 369.1677, found 369.1672 (+1.4 ppm).

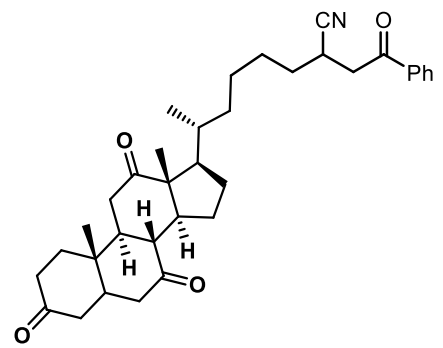

6-((5S,8R,9S,10S,13R,14S,17R)-10,13-dimethyl3,7,12trioxohexadecahydro-1H-cyclopenta[a]phenanthren-17-yl)-2(2-oxo-2-phenylethyl)heptanenitrile (9d): Prepared according to General Procedure I using tert-butyldimethyl((1phenylvinyl)oxy)silane $\quad 7 \mathbf{a} \quad(63 \quad \mathrm{mg}, \quad 0.24 \quad \mathrm{mmol}), \quad 1,3-$ dioxoisoindolin-2-yl 4-((5S,8R,9S,10S,13R,14S,17R)-10,13dimethyl-3,7,12-trioxohexadecahydro- $1 \mathrm{H}$ cyclopenta[a]phenanthren-17-yl)pentanoate $\mathbf{1 d}(110 \mathrm{mg}, 0.2$ $\mathrm{mmol}$ ), and acrylonitrile $\mathbf{2 b}(22 \mu \mathrm{L}, 0.340 \mathrm{mmol})$. Flash column chromatography (hexanes/EtOAc 1:1) to afford product $9 \mathbf{d}$ as a white solid (64 $\mathrm{mg}, 60 \%$ yield).

${ }^{1} \mathrm{H} \mathrm{NMR}\left(300 \mathrm{MHz}, \mathrm{CDCl}_{3}\right) \delta 8.03-7.89(\mathrm{~m}, 2 \mathrm{H}), 7.72-7.57(\mathrm{~m}, 1 \mathrm{H}), 7.53-7.45(\mathrm{~m}, 2 \mathrm{H})$, $3.63-3.15(\mathrm{~m}, 3 \mathrm{H}), 2.99-2.75(\mathrm{~m}, 3 \mathrm{H}), 2.39-1.80(\mathrm{~m}, 14 \mathrm{H}), 1.77-1.43(\mathrm{~m}, 6 \mathrm{H}), 1.40(\mathrm{~s}, 3 \mathrm{H})$, $1.35-1.16(\mathrm{~m}, 3 \mathrm{H}), 1.07$ (s, 3H), $0.86(\mathrm{dd}, J=6.4,2.0 \mathrm{~Hz}, 3 \mathrm{H})$.

${ }^{13} \mathrm{C} \mathrm{NMR}\left(75 \mathrm{MHz}, \mathrm{CDCl}_{3}\right) \delta 212.0,209.1,208.8,195.3,135.9,133.9,128.9,128.1,121.9,56.9$, 51.8, 49.0, 46.9, 45.8, 45.6, 45.0, 42.8, 40.9, 40.8, 38.7, 36.5, 36.0, 36.0, 35.3, 34.8, 32.4, 29.7, 27.9, 26.4, 26.3, 25.2, 24.5, 24.4, 21.9, 19.0, 18.9, 11.9.

HRMS: calculated for $\mathrm{C}_{34} \mathrm{H}_{43} \mathrm{NNaO}_{4}\left(\mathrm{M}+\mathrm{Na}^{+}\right)$: 552.3081, found $552.3084(-0.5 \mathrm{ppm})$.

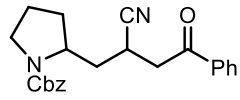

Benzyl 2-(2-cyano-4-oxo-4-phenylbutyl)pyrrolidine-1-carboxylate (9e): Prepared according to General Procedure I using tert-butyldimethyl((1phenylvinyl)oxy)silane $7 \mathbf{7 a}(63 \mathrm{mg}, \quad 0.240 \mathrm{mmol})$, 1-benzyl 2-(1,3dioxoisoindolin-2-yl) pyrrolidine-1,2-dicarboxylate 1c $(55 \mathrm{mg}, 0.2 \mathrm{mmol})$, and acrylonitrile $\mathbf{2 b}$ $(22 \mu \mathrm{L}, 0.34 \mathrm{mmol}$ ). Flash column chromatography (hexanes/acetone 7:3) to afford product $9 \mathrm{e}$ as a reddish oil (47 $\mathrm{mg}, 62 \%$ yield, 1:1 dr). 
${ }^{1} \mathrm{H}$ NMR (400 MHz, $\left.\mathrm{CDCl}_{3}\right)(1: 1$ mixture of diastereisomers) $\delta 8.01-7.79(\mathrm{~m}, 2 \mathrm{H}), 7.68-7.59$ $(\mathrm{m}, 1 \mathrm{H}), 7.50(\mathrm{t}, J=7.6 \mathrm{~Hz}, 2 \mathrm{H}), 7.46-7.30(\mathrm{~m}, 5 \mathrm{H}), 5.26-5.10(\mathrm{~m}, 2 \mathrm{H}), 4.24-3.96(\mathrm{~m}, 1 \mathrm{H})$, $3.57-3.19(\mathrm{~m}, 5 \mathrm{H}), 2.15-2.04(\mathrm{~m}, 1 \mathrm{H}), 2.02-1.65(\mathrm{~m}, 5 \mathrm{H})$.

${ }^{13} \mathrm{C}$ NMR (101 MHz, $\mathrm{CDCl}_{3}$ ) (1:1 mixture of diastereisomers) $\delta 195.3,194.8,155.3,136.8$, $136.8,133.8,128.8,128.5,128.1,127.8,123.7,122.0,121.166 .9,66.8,56.2,55.6,55.346 .4$, 41.0, 40.7, 36.9, 36.5, 36.0, 32.5, 30.6, 30.3, 29.7, 24.3, 23.9, 23.4, 23.0.

HRMS: calculated for $\mathrm{C}_{23} \mathrm{H}_{24} \mathrm{~N}_{2} \mathrm{NaO}_{3}\left(\mathrm{M}+\mathrm{Na}^{+}\right)$: 399.1679 , found 399.1682 (-0.75 ppm).

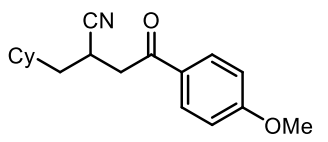

2-(cyclohexylmethyl)-4-(4-methoxyphenyl)-4-oxobutanenitrile (9f): Prepared according to General Procedure I using tert-butyl((1-(4methoxyphenyl)vinyl)oxy)dimethylsilane $7 \mathrm{e}(65 \mathrm{mg}, 0.24 \mathrm{mmol}), 1,3-$ dioxoisoindolin-2-yl cyclohexanecarboxylate $1 \mathrm{a}(55 \mathrm{mg}, 0.2 \mathrm{mmol})$, and acrylonitrile $(22 \mu \mathrm{L}, 0.34 \mathrm{mmol})$. Flash column chromatography (hexanes/acetone 88:12) to afford product $9 f$ as an off-white solid (31 $\mathrm{mg}, 61 \%$ yield).

${ }^{1} \mathrm{H} \mathrm{NMR}\left(300 \mathrm{MHz}, \mathrm{CDCl}_{3}\right) \delta 7.93(\mathrm{~d}, J=8.9 \mathrm{~Hz}, 2 \mathrm{H}), 6.95(\mathrm{~d}, J=8.9 \mathrm{~Hz}, 2 \mathrm{H}), 3.88(\mathrm{~s}, 3 \mathrm{H})$, $3.42-3.29(\mathrm{~m}, 2 \mathrm{H}), 3.21-3.10(\mathrm{~m}, 1 \mathrm{H}), 1.88(\mathrm{~d}, J=12.8 \mathrm{~Hz}, 1 \mathrm{H}), 1.77-1.58(\mathrm{~m}, 7 \mathrm{H}), 1.23-$ $1.10(\mathrm{~m}, 2 \mathrm{H}), 1.00-0.79(\mathrm{~m}, 3 \mathrm{H})$.

${ }^{13} \mathrm{C} \mathrm{NMR}\left(75 \mathrm{MHz}, \mathrm{CDCl}_{3}\right) \delta 193.9,164.2,130.5 .0,129.2,122.4,114.1,55.7,41.1,39.9,35.7$, 33.8, 32.2, 26.5, 26.2, 26.0, 24.0.

HRMS: calculated for $\mathrm{C}_{18} \mathrm{H}_{23} \mathrm{NNaO}_{2}\left(\mathrm{M}+\mathrm{Na}^{+}\right)$: 308.1630, found 308.1621 (+2.9 ppm). 


\section{C5. Minisci Reaction}

\section{C5.1 General Procedure J}
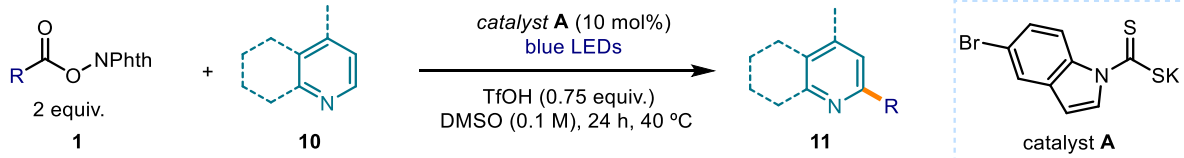

Reactions performed using set-up 1 in Figure S2. In an oven dried vial, with a Teflon septum screw cap, heteroarene 10 (0.2 mmol, 1.0 equiv.) and trifluoromethanesulfonic acid $(13.3 \mu \mathrm{L}$, $0.15 \mathrm{mmol}, 0.75$ equiv.) were dissolved in DMSO $(2 \mathrm{ml})$. Then, phthalimide ester $1(0.4 \mathrm{mmol}, 2$ equiv.) and catalyst $\mathbf{A}(6.2 \mathrm{mg}, 0.02 \mathrm{mmol}, 0.1$ equiv.) were added. The resulting yellow mixture was degassed with argon sparging for 60 seconds. The vial was placed in the 3D printed photoreactor and irradiated under stirring for 24 hours, unless otherwise specified (Figure S2). The mixture was transferred to an extraction funnel, $\mathrm{NaHCO}_{3}$ sat. solution was added and the organic layer was extracted with EtOAc. The organic layer was washed with brine twice. The combined organic layers were dried over anhydrous $\mathrm{MgSO}_{4}$, filtered, and concentrated to dryness. The crude residue was purified by column chromatography to afford the corresponding product in the stated yield with $>95 \%$ purity according to ${ }^{1} \mathrm{H}$ NMR analysis.

\section{C5.2 Characterization of Products with General Procedure J}<smiles>Cc1cc(C)c2ccccc2n1</smiles>

2-Cyclohexyl-4-methylquinoline(11a): Synthesized according to General Procedure J using 1,3-dioxoisoindolin-2-yl cyclohexanecarboxylate 1a (109 mg, $0.4 \mathrm{mmol}, 2$ equiv.) and 4-methylquinoline 10a $(26.4 \mu \mathrm{L}, 0.2 \mathrm{mmol}, 1.0$ equiv.). The crude mixture was purified by flash column chromatography on silica gel (10\% EtOAc in hexane as eluent) to afford 11a ( $39.6 \mathrm{mg}, 88 \%$ yield) as a yellow oil.

${ }^{1} \mathrm{H} \mathrm{NMR}\left(400 \mathrm{MHz}, \mathrm{CDCl}_{3}\right) \delta 8.28(\mathrm{~s}, 1 \mathrm{H}), 7.98-7.96(\mathrm{~m}, 1 \mathrm{H}), 7.74-7.70(\mathrm{~m}, 1 \mathrm{H}), 7.57-7.53$ $(\mathrm{m}, 1 \mathrm{H}), 7.23(\mathrm{~s}, 1 \mathrm{H}), 3.15-3.10(\mathrm{~m}, 1 \mathrm{H}), 2.73(\mathrm{~s}, 3 \mathrm{H}), 2.06-2.01(\mathrm{~m}, 2 \mathrm{H}), 1.89(\mathrm{dt}, J=13.0$, $3.3 \mathrm{~Hz}, 2 \mathrm{H}), 1.83-1.76(\mathrm{~m}, 1 \mathrm{H}), 1.63(\mathrm{qd}, J=12.3,3.0 \mathrm{~Hz}, 2 \mathrm{H}), 1.49$ (qt, $J=12.8,3.0 \mathrm{~Hz}, 2 \mathrm{H}$ ), $1.38-1.30(\mathrm{~m}, 1 \mathrm{H})$.

${ }^{13} \mathrm{C} \mathrm{NMR}\left(101 \mathrm{MHz}, \mathrm{CDCl}_{3}\right) \delta 166.0,130.2,128.1,127.1,126.3,123.8,120.3,46.5,32.9,26.5$, 26.1, 19.3.

Matching reported literature data. ${ }^{27}$<smiles>Cc1cc(Cl)c2ccccc2n1</smiles>

4-Cyclohexyl-2-methylquinoline (11b): Synthesized according to General Procedure J using 1,3-dioxoisoindolin-2-yl cyclohexanecarboxylate 1a (109 mg, 0.4 mmol, 2 equiv.) and 2-methylquinoline $\mathbf{1 0 b}(27.1 \mu \mathrm{L}, 0.2 \mathrm{mmol}, 1.0$ equiv.). The crude mixture was purified by flash column chromatography on silica gel $(10 \%$ EtOAc in hexane as eluent) to afford $\mathbf{1 1 b}(42.3 \mathrm{mg}, 94 \%$ yield $)$ as a yellow oil.

${ }^{1} \mathrm{H} \mathrm{NMR}\left(400 \mathrm{MHz}, \mathrm{CDCl}_{3}\right) \delta 8.05(\mathrm{t}, J=9.8 \mathrm{~Hz}, 2 \mathrm{H}), 7.67-7.63(\mathrm{~m}, 1 \mathrm{H}), 7.51-7.47(\mathrm{~m}, 1 \mathrm{H})$, $7.17(\mathrm{~s}, 1 \mathrm{H}), 3.32-3.27(\mathrm{ddd}, J=11.4,8.3,3.4 \mathrm{~Hz}, 1 \mathrm{H}), 2.73(\mathrm{~s}, 3 \mathrm{H}), 2.05-1.90(\mathrm{~m}, 4 \mathrm{H}), 1.88$ - $1.83(\mathrm{~m}, 1 \mathrm{H}), 1.61-1.48(\mathrm{~m}, 4 \mathrm{H}), 1.40-1.31(\mathrm{~m}, 4 \mathrm{H})$.

${ }^{13} \mathrm{C} \mathrm{NMR}\left(101 \mathrm{MHz}, \mathrm{CDCl}_{3}\right) \delta 158.8,153.8,147.9,129.4,129.1,125.5,125.3,123.0,118.5,39.0$, 33.7, 27.1, 26.5, 25.5.

Matching reported literature data. ${ }^{27}$

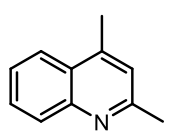

2,4-Dimethylquinoline (11c): Synthesized according to General Procedure J using 1,3-dioxoisoindolin-2-yl acetate $\mathbf{1 g}(82.1 \mathrm{mg}, 0.4 \mathrm{mmol}, 2$ equiv.) and 2methylquinoline 10b $(27.1 \mu \mathrm{L}, 0.2 \mathrm{mmol}, 1.0$ equiv.) in NMP $(2 \mathrm{ml})$ for 48 hours. The crude mixture was purified by flash column chromatography on silica gel ( $20 \%$

$\mathrm{CH}_{2} \mathrm{Cl}_{2}$ in $\mathrm{MeOH}$ as eluent) to afford $11 \mathrm{c}(28.3 \mathrm{mg}, 90 \%$ yield) as a colorless oil. 
${ }^{1} \mathrm{H} \mathrm{NMR}\left(400 \mathrm{MHz}, \mathrm{CDCl}_{3}\right) \delta 8.09(\mathrm{dt}, J=8.5,0.9 \mathrm{~Hz}, 1 \mathrm{H}), 7.89-7.85(\mathrm{~m}, 1 \mathrm{H}), 7.72(\mathrm{ddd}, J=$ 8.4, 6.9, 1.4 Hz, 1H), 7.54 (ddd, $J=8.3,6.9,1.3 \mathrm{~Hz}, 1 \mathrm{H}), 6.98(\mathrm{~s}, 1 \mathrm{H}), 2.86$ (s, 3H), 2.73 (s, 3H).

${ }^{13} \mathrm{C} \mathrm{NMR}\left(101 \mathrm{MHz}, \mathrm{CDCl}_{3}\right) \delta 159.2,148.6,129.9,129.7,126.4,124.1,123.4,122.1,29.8,25.6$. Matching reported literature data. ${ }^{28}$

$\overbrace{C y}^{N}$

1-Cyclohexylisoquinoline (11d): Synthesized according to General Procedure J using 1,3-dioxoisoindolin-2-yl cyclohexanecarboxylate 1a (109 mg, $0.4 \mathrm{mmol}, 2$ equiv.) and isoquinoline $10 \mathrm{c}(25.8 \mathrm{mg}, 0.2 \mathrm{mmol}, 1.0$ equiv.). The crude mixture was purified by flash column chromatography on silica gel (10\% EtOAc in hexane as eluent) to afford 11d (40.9 mg, $97 \%$ yield) as a colorless oil.

${ }^{1} \mathrm{H} \mathrm{NMR}\left(400 \mathrm{MHz}, \mathrm{CDCl}_{3}\right) \delta 8.50(\mathrm{~d}, J=5.8 \mathrm{~Hz}, 1 \mathrm{H}), 8.25(\mathrm{~d}, J=8.5 \mathrm{~Hz}, 1 \mathrm{H}), 7.83(\mathrm{~d}, J=8.3$ $\mathrm{Hz}, 1 \mathrm{H}), 7.72-7.66(\mathrm{~m}, 1 \mathrm{H}), 7.63-7.59(\mathrm{ddd}, J=8.3,6.9,1.4 \mathrm{~Hz}, 1 \mathrm{H}), 7.53(\mathrm{~d}, J=5.8 \mathrm{~Hz}, 1 \mathrm{H})$, $3.58(\mathrm{tt}, J=11.6,3.2 \mathrm{~Hz}, 1 \mathrm{H}), 2.05-1.78(\mathrm{~m}, 7 \mathrm{H}), 1.61-1.37(\mathrm{~m}, 3 \mathrm{H})$.

${ }^{13} \mathrm{C} \mathrm{NMR}\left(101 \mathrm{MHz}, \mathrm{CDCl}_{3}\right) \delta 165.7,141.1,136.8,130.3,127.8,127.3,126.4,125.1,119.4,41.6$, 32.7, 27.0, 26.3.

Matching reported literature data. ${ }^{27}$<smiles>Cc1cc(C)nc(Cl)c1</smiles>

2-Cyclohexyl-4,6-dimethylpyridine (11e): Synthesized according to General Procedure J using 1,3-dioxoisoindolin-2-yl acetate 1a (109 mg, $0.4 \mathrm{mmol}, 2$ equiv) and 2,4-dimethylpyridine 10d $(23.1 \mu \mathrm{L}, 0.2 \mathrm{mmol}, 1.0$ equiv.). The crude mixture was purified by flash column chromatography on silica gel (10\% EtOAc in hexane as eluent) to afford $11 e(31.3 \mathrm{mg}, 83 \%$ yield) as a colorless oil.

${ }^{1} \mathrm{H}$ NMR $\left(400 \mathrm{MHz}, \mathrm{CDCl}_{3}\right) \delta 6.80(\mathrm{~s}, 2 \mathrm{H}), 2.71(\mathrm{dd}, J=8.5,4.8 \mathrm{~Hz}, 1 \mathrm{H}), 2.50(\mathrm{~s}, 3 \mathrm{H}), 2.29(\mathrm{~s}$, $3 \mathrm{H}), 1.95(\mathrm{dd}, J=13.4,3.0 \mathrm{~Hz}, 2 \mathrm{H}), 1.86-1.79(\mathrm{~m}, 2 \mathrm{H}), 1.78-1.70(\mathrm{~m}, 1 \mathrm{H}), 1.51-1.36(\mathrm{~m}$, $4 \mathrm{H}), 1.33-1.25(\mathrm{~m}, 1 \mathrm{H})$.

${ }^{13} \mathrm{C} \mathrm{NMR}\left(101 \mathrm{MHz}, \mathrm{CDCl}_{3}\right) \delta 165.8,157.0,122.0,118.6,44.1,33.3,26.7,26.3,23.1,21.2$.

Matching reported literature data. ${ }^{27}$<smiles>[B]c1ccc2nc([Hg])sc2c1</smiles>

6-Bromo-2-cyclohexylbenzo[d]thiazole (11f): Synthesized according to General Procedure J using 1,3-dioxoisoindolin-2-yl cyclohexanecarboxylate 1a (109 mg, $0.4 \mathrm{mmol}, 2$ equiv.) and 6-bromo-1,3-benzothiazole 10e $(42.8 \mathrm{mg}, 0.2$ mmol, 1.0 equiv.). The crude mixture was purified by flash column chromatography on silica gel (10\% AcOEt in hexane as eluent) to afford $\mathbf{1 1 f}(49.1 \mathrm{mg}, 83 \%$ yield) as a white soild.

${ }^{1} \mathrm{H} \mathrm{NMR}\left(500 \mathrm{MHz}, \mathrm{CDCl}_{3}\right) \delta 7.96(\mathrm{~d}, J=2.0 \mathrm{~Hz}, 1 \mathrm{H}), 7.81(\mathrm{~d}, J=8.7 \mathrm{~Hz}, 1 \mathrm{H}), 7.53(\mathrm{dd}, J=$ $8.7,2.0 \mathrm{~Hz}, 1 \mathrm{H}), 3.07(\mathrm{tt}, J=11.6,3.6 \mathrm{~Hz}, 1 \mathrm{H}), 2.22-2.15(\mathrm{~m}, 2 \mathrm{H}), 1.88(\mathrm{dp}, J=10.6,3.5 \mathrm{~Hz}$, $2 \mathrm{H}), 1.76(\mathrm{dtt}, J=13.1,3.4,1.5 \mathrm{~Hz}, 1 \mathrm{H}), 1.69-1.57(\mathrm{~m}, 2 \mathrm{H}), 1.50-1.37(\mathrm{~m}, 2 \mathrm{H}), 1.32(\mathrm{tt}, J=$ $12.5,3.5 \mathrm{~Hz}, 1 \mathrm{H})$.

${ }^{13} \mathrm{C} \mathrm{NMR}\left(126 \mathrm{MHz}, \mathrm{CDCl}_{3}\right) \delta 178.3,152.1,136.4,129.4,124.2,123.8,118.2,43.5,33.5,26.1$, 25.9.

Matching reported literature data. ${ }^{28}$

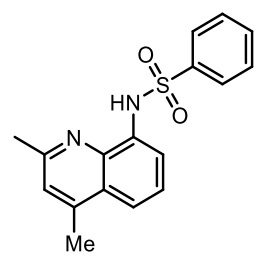

$N$-(2,4-Dimethylquinolin-8-yl) benzenesulfonamide (11g): Synthesized according to General Procedure J using 1,3-dioxoisoindolin-2-yl acetate 1g (82.1 mg, $0.4 \mathrm{mmol}, 2.0$ equiv.) and 2-((4-((7-chloro-2-methylquinolin-4-yl) amino) pentyl) (ethyl) amino) ethan-1-ol $10 f(59.7 \mathrm{mg}, 0.2 \mathrm{mmol}, 1.0$ equiv.) in NMP $(2 \mathrm{ml}, 0.1 \mathrm{M})$ for 48 hours. The crude mixture was purified by flash column chromatography on silica gel $\left(20 \% \mathrm{CH}_{2} \mathrm{Cl}_{2}\right.$ in $\mathrm{MeOH}$ as eluent) to afford $11 \mathrm{~g}$ (37.5 $\mathrm{mg}, 60 \%$ yield) as a white soild. 
${ }^{1} \mathrm{H} \mathrm{NMR}\left(300 \mathrm{MHz}, \mathrm{CDCl}_{3}\right) \delta 9.48(\mathrm{~s}, 1 \mathrm{H}), 8.01-7.91(\mathrm{~m}, 2 \mathrm{H}), 7.80(\mathrm{dd}, J=7.3,1.5 \mathrm{~Hz}, 1 \mathrm{H})$, $7.53-7.36(\mathrm{~m}, 5 \mathrm{H}), 6.98(\mathrm{~s}, 1 \mathrm{H}), 2.82(\mathrm{~s}, 3 \mathrm{H}), 2.70(\mathrm{~s}, 4 \mathrm{H})$.

${ }^{13} \mathrm{C} \mathrm{NMR}\left(101 \mathrm{MHz}, \mathrm{CDCl}_{3}\right) \delta 176.0,158.0,139.6,138.2,134.0,133.0,129.1,127.4,126.7$, 124.2, 116.7, 115.4, 29.1, 25.3.

HRMS: calculated for $\mathrm{C}_{17} \mathrm{H}_{16} \mathrm{~N}_{2} \mathrm{NaO}_{2} \mathrm{~S}\left(\mathrm{M}+\mathrm{Na}^{+}\right)$: 335.3762 , found: 335.3768 .<smiles>COC(=O)c1nc(Cl)c2cc(-c3ccccc3)ccc2c1O</smiles>

Methyl 1-cyclohexyl-4-hydroxy-7-phenoxyisoquinoline-3-carboxylate (11h): Synthesized according to General Procedure J using 1,3dioxoisoindolin-2-yl cyclohexanecarboxylate $1 \mathrm{a}(109 \mathrm{mg}, 0.4 \mathrm{mmol}, 2.0$ equiv.,) and Roxadustat analogue $10 \mathrm{~g}(59.1 \mathrm{mg}, 0.2 \mathrm{mmol}, 1.0$ equiv.). The crude mixture was purified by flash column chromatography on silica gel (50\% EtOAc in hexane as eluent) to afford $\mathbf{1 1 h}(67.1 \mathrm{mg}, 89 \%$ yield) as a white soild.

${ }^{1} \mathrm{H}$ NMR $\left(400 \mathrm{MHz}, \mathrm{CDCl}_{3}\right) \delta 8.41(\mathrm{~d}, J=9.1 \mathrm{~Hz}, 1 \mathrm{H}), 7.62(\mathrm{~d}, J=2.4 \mathrm{~Hz}, 1 \mathrm{H}), 7.49-7.41(\mathrm{~m}$, $3 \mathrm{H}), 7.27-7.21(\mathrm{~m}, 1 \mathrm{H}), 7.17-7.10(\mathrm{~m}, 2 \mathrm{H}), 4.08(\mathrm{~s}, 3 \mathrm{H}), 3.18(\mathrm{tt}, J=11.6,3.3 \mathrm{~Hz}, 1 \mathrm{H}), 1.99$ $-1.85(\mathrm{~m}, 4 \mathrm{H}), 1.86-1.71(\mathrm{~m}, 3 \mathrm{H}), 1.51-1.31(\mathrm{~m}, 3 \mathrm{H})$.

${ }^{13} \mathrm{C} \mathrm{NMR}\left(101 \mathrm{MHz}, \mathrm{CDCl}_{3}\right) \delta 171.8,159.0,156.1,155.4,155.4,131.4,130.3,126.2,124.6$, 124.2, 121.9, 119.8, 119.0, 111.4, 53.0, 41.9, 32.2, 26.8, 26.2.

HRMS: calculated for $\mathrm{C}_{23} \mathrm{H}_{24} \mathrm{NO}_{4}\left(\mathrm{M}+\mathrm{H}^{+}\right)$: 378.1700 , found: 378.1702 .<smiles>CC(=O)c1nc(C)c2cc(O)ccc2c1O</smiles>

Methyl 4-hydroxy-1-methyl-7-phenoxyisoquinoline-3-carboxylate (11i): Synthesized according to General Procedure $\mathrm{J}$ using 1,3dioxoisoindolin-2-yl acetate $1 \mathrm{~g}(82.1 \mathrm{mg}, 0.4 \mathrm{mmol}, 2.0$ equiv.) and Roxadustat analogue $\mathbf{1 0 g}(59.1 \mathrm{mg}, 0.2 \mathrm{mmol}, 1.0$ equiv.) in NMP (2 ml) for 48 hours. The crude mixture was purified by flash column chromatography on silica gel $(50 \%$ EtOAc in hexane as eluent) to afford $\mathbf{1 1 i}$ (57.5 mg, 93\% yield) as a white soild.

$\underline{{ }^{1} \mathrm{H} \mathrm{NMR}}\left(300 \mathrm{MHz}, \mathrm{CDCl}_{3}\right) \delta 11.83(\mathrm{~s}, 1 \mathrm{H}), 8.44(\mathrm{~d}, J=9.1 \mathrm{~Hz}, 1 \mathrm{H}), 7.53-7.43(\mathrm{~m}, 3 \mathrm{H}), 7.38$ (m, 1H), $7.29(\mathrm{tt}, J=6.4,1.2 \mathrm{~Hz}, 1 \mathrm{H}), 7.16-7.09(\mathrm{~m}, 2 \mathrm{H}), 4.04(\mathrm{~s}, 3 \mathrm{H}), 2.71(\mathrm{~s}, 3 \mathrm{H})$.

${ }^{13} \mathrm{C}$ NMR $\left(101 \mathrm{MHz}, \mathrm{CDCl}_{3}\right) \delta 175.4,160.1,156.4,155.2,147.5,130.7,130.5,126.7,125.4$, 124.2, 122.4, 120.4, 119.1, 108.6, 53.0, 30.0.

Matching reported literature data. ${ }^{29}$

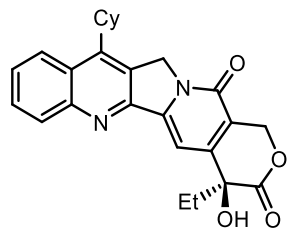

(S)-11-Cyclohexyl-4-ethyl-4-hydroxy-1,12-dihydro-14H-pyrano $\left[3^{\prime}, 4^{\prime}: 6,7\right]$ indolizino [1,2-b] quinoline-3,14(4H)-dione (11j): Synthesized according to General Procedure using J 1,3-dioxoisoindolin-2-yl cyclohexanecarboxylate 1a (109 mg, $0.4 \mathrm{mmol}, 2$ equiv.) and Camptothecin 10h $(69.7 \mathrm{mg}, 0.2 \mathrm{mmol}, 1.0$ equiv.). The crude mixture was purified by flash column chromatography on silica gel ( $5 \% \mathrm{CH}_{2} \mathrm{Cl}_{2}$ in $\mathrm{MeOH}$ as eluent) to afford $\mathbf{1 1 j}$ (41,3 $\mathrm{mg}, 48 \%$ yield) as a white soild.

${ }^{1} \mathrm{H} \mathrm{NMR}\left(400 \mathrm{MHz}, \mathrm{CDCl}_{3}\right) \delta 8.27-8.15(\mathrm{~d}, J=8.4 \mathrm{~Hz}, 2 \mathrm{H}), \delta 7.76(\mathrm{t}, J=7.5 \mathrm{~Hz}, 1 \mathrm{H}), 7.66-$ $7.61(\mathrm{~m}, 2 \mathrm{H}), 5.74(\mathrm{~d}, J=16.3 \mathrm{~Hz}, 1 \mathrm{H}), 5.39(\mathrm{~s}, 2 \mathrm{H}), 5.29$ (d, $J=16.3 \mathrm{~Hz}, 1 \mathrm{H}), 3.95(\mathrm{~s}, 1 \mathrm{H}), 3.61$ (s, 1H), $2.06-1.82(\mathrm{~m}, 8 \mathrm{H}), 1.66-1.53(\mathrm{~m}, 3 \mathrm{H}), 1.46(\mathrm{t}, J=12.7 \mathrm{~Hz}, 1 \mathrm{H}), 1.03(\mathrm{t}, J=7.4 \mathrm{~Hz}$, $3 \mathrm{H})$.

${ }^{13} \mathrm{C} \mathrm{NMR}\left(101 \mathrm{MHz}, \mathrm{CDCl}_{3}\right) \delta 174.1,157.7,150.4,148.8,130.9,130.0,127.8,127.1,118.5,97.9$, $72.9,66.5,50.8,31.9,31.8,29.8,27.1,26.1,8.0$.

Matching reported literature data. ${ }^{30}$ 


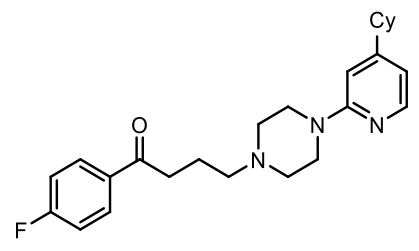

4-(4-(4-Cyclohexylpyridin-2-yl)

piperazin-1-yl)-1-(4-

fluorophenyl) butan-1-one (11k): Synthesized according to General Procedure J using 1,3-dioxoisoindolin-2-yl cyclohexanecarboxylate 1a $(109 \mathrm{mg}, 0.4 \mathrm{mmol}, 2.0$ equiv.) and Azaperone 10i (65.5 mg, $0.2 \mathrm{mmol}, 1.0$ equiv.). The crude mixture was purified by flash column chromatography on silica gel $(50 \%$

${ }^{1} \mathrm{H}$ NMR $\left(400 \mathrm{MHz}\right.$, Acetone- $\left.d_{6}\right) \delta 8.15-8.07(\mathrm{~m}, 2 \mathrm{H}), 7.46(\mathrm{dd}, J=8.4,7.3 \mathrm{~Hz}, 1 \mathrm{H}), 7.30-$ $7.24(\mathrm{~m}, 2 \mathrm{H}), 6.63(\mathrm{~d}, J=8.4 \mathrm{~Hz}, 1 \mathrm{H}), 6.54(\mathrm{~d}, J=7.4 \mathrm{~Hz}, 1 \mathrm{H}), 3.65-3.53(\mathrm{~m}, 4 \mathrm{H}), 3.19(\mathrm{t}, J=$ $6.9 \mathrm{~Hz}, 2 \mathrm{H}), 3.00-2.90(\mathrm{~m}, 5 \mathrm{H}), 2.52(\mathrm{tt}, J=11.8,3.4 \mathrm{~Hz}, 1 \mathrm{H}), 2.15-2.07(\mathrm{~m}, 2 \mathrm{H}), 1.83-1.28$ $(\mathrm{m}, 10 \mathrm{H})$.

${ }^{13} \mathrm{C}$ NMR $\left(101 \mathrm{MHz}\right.$, Acetone- $\left.d_{6}\right) \delta 198.7,165.1,159.5,138.7,131.8,131.7,116.4,116.2,111.1$, 105.1, 58.1, 53.5, 47.1, 45.1, 36.4, 33.5, 30.6, 27.3, 26.9.

${ }^{19} \mathrm{~F} \mathrm{NMR}\left(376 \mathrm{MHz}\right.$, Acetone- $\left.d_{6}\right) \delta-108.12$.

HRMS: calculated for $\mathrm{C}_{25} \mathrm{H}_{33} \mathrm{FN}_{3} \mathrm{O}\left(\mathrm{M}+\mathrm{H}^{+}\right)$: 410.2602 , found 410.2595 (-1.7 ppm).<smiles>Cc1cc(CCCc2ccccc2)nc2ccccc12</smiles>

4-Methyl-2-(3-phenylpropyl)quinoline (11): Synthesized according to General Procedure J using 1,3-dioxoisoindolin-2-yl 4-phenylbutanoate 1b (123 mg, $0.4 \mathrm{mmol}, 2.0$ equiv.) and 4-methylquinoline 10a $(26.4 \mu \mathrm{L}, 0.2$ mmol, 1.0 equiv.). The crude mixture was purified by flash column chromatography on silica gel (10\% EtOAc in hexane as eluent) to afford $\mathbf{1 1 l}(46.0 \mathrm{mg}, 88 \%$ yield) as a white soild.

${ }^{1} \mathrm{H}$ NMR $\left(400 \mathrm{MHz}, \mathrm{CDCl}_{3}\right) \delta 8.11-8.03(\mathrm{~m}, 1 \mathrm{H}), 7.95(\mathrm{dd}, J=8.3,1.4 \mathrm{~Hz}, 1 \mathrm{H}), 7.68(\mathrm{ddd}, J=$ 8.4, 6.9, $1.4 \mathrm{~Hz}, 1 \mathrm{H}), 7.51$ (ddd, $J=8.2,6.9,1.3 \mathrm{~Hz}, 1 \mathrm{H}), 7.33-7.26(\mathrm{~m}, 2 \mathrm{H}), 7.26-7.15(\mathrm{~m}$, $3 \mathrm{H}), 7.13(\mathrm{~d}, J=1.1 \mathrm{~Hz}, 1 \mathrm{H}), 3.03-2.95(\mathrm{~m}, 2 \mathrm{H}), 2.79-2.71(\mathrm{~m}, 2 \mathrm{H}), 2.67(\mathrm{~d}, J=1.0 \mathrm{~Hz}, 3 \mathrm{H})$, $2.21-2.10(\mathrm{~m}, 2 \mathrm{H})$.

${ }^{13} \mathrm{C} \mathrm{NMR}\left(101 \mathrm{MHz}, \mathrm{CDCl}_{3}\right) \delta 162.3,147.7,144.6,142.3,129.4,129.3,128.6,128.4,126.9$, 125.9, 125.7, 123.7, 122.2, 38.8, 35.9, 31.7, 18.8 .

HRMS: calculated for $\mathrm{C}_{19} \mathrm{H}_{20} \mathrm{~N}\left(\mathrm{M}+\mathrm{H}^{+}\right)$: 262.1586, found 262.1590 (+1.5 ppm).

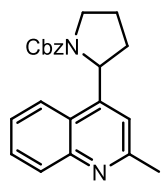

Benzyl 2-(2-methylquinolin-4-yl)pyrrolidine-1-carboxylate (11m): Synthesized according to General Procedure J using 1-benzyl 2-(1,3-dioxoisoindolin-2-yl) pyrrolidine-1,2-dicarboxylate $1 \mathrm{c}(157 \mathrm{mg}, 0.4 \mathrm{mmol}, 2$ equiv. $)$ and 2methylquinoline 10b $(27.1 \mu \mathrm{L}, 0.2 \mathrm{mmol}, 1.0$ equiv.). The crude mixture was purified by flash column chromatography on silica gel (30\% EtOAc in hexane as eluent) to afford $11 \mathrm{~m}(57.3 \mathrm{mg}, 83 \%$ yield) as a yellow oil.

${ }^{1} \mathrm{H} \mathrm{NMR}\left(400 \mathrm{MHz}, \mathrm{CDCl}_{3}\right)$ mixture of rotamers: $\delta 8.08(\mathrm{t}, J=9.5 \mathrm{~Hz}, 1 \mathrm{H}), 7.92(\mathrm{dd}, J=8.8,4.0$ $\mathrm{Hz}, 1 \mathrm{H}), 7.67(\mathrm{dt}, J=12.4,7.6 \mathrm{~Hz}, 1 \mathrm{H}), 7.50(\mathrm{t}, J=7.5 \mathrm{~Hz}, 1 \mathrm{H}), 7.44-7.28(\mathrm{~m}, 2 \mathrm{H}), 7.11(\mathrm{dt}, J$ $=14.7,7.2 \mathrm{~Hz}, 2 \mathrm{H}), 6.98(\mathrm{~d}, J=3.3 \mathrm{~Hz}, 1 \mathrm{H}), 6.85(\mathrm{~d}, J=7.4 \mathrm{~Hz}, 1 \mathrm{H}), 5.76-5.61(\mathrm{~m}, 1 \mathrm{H}), 5.18$ $(\mathrm{s}, 1 \mathrm{H}), 5.10-4.83(\mathrm{~m}, 1 \mathrm{H}), 3.82(\mathrm{td}, J=9.5,8.2,3.1 \mathrm{~Hz}, 1 \mathrm{H}), 3.77-3.62(\mathrm{~m}, 1 \mathrm{H}), 2.67(\mathrm{~d}, J=$ $26.7 \mathrm{~Hz}, 3 \mathrm{H}), 2.59-2.40(\mathrm{~m}, 1 \mathrm{H}), 2.00-1.79(\mathrm{~m}, 3 \mathrm{H})$.

${ }^{13} \mathrm{C}$ NMR $\left(101 \mathrm{MHz}, \mathrm{CDCl}_{3}\right)$ mixture of rotamers: $\delta 158.8,158.6,154.9,149.3,148.6,148.0$, 136.9, 136.5, 129.5, 129.3, 128.6, 128.3, 128.2, 128.1, 127.8, 127.4, 125.8, 124.1, 124.0, 123.0, $122.8,117.6,117.3,67.2,66.7,58.1,57.5,47.6,47.3,34.2,33.2,25.5,23.7,23.0$.

HRMS: calculated for $\mathrm{C}_{22} \mathrm{H}_{23} \mathrm{~N}_{2} \mathrm{O}_{2}\left(\mathrm{M}+\mathrm{H}^{+}\right)$: 347.1754 , found 347.1760 (-1.7 ppm). 
2-Methyl-4-(1-tosylpiperidin-4-yl)quinoline (11n): Synthesized according to General Procedure J using 1,3-dioxoisoindolin-2-yl 1-tosylpiperidine-4-carboxylate 1f (171 mg, $0.4 \mathrm{mmol}, 2.0$ equiv.) and 2-methylquinoline $\mathbf{1 0 b}(27.1 \mu \mathrm{L}, 0.2 \mathrm{mmol}$, 1.0 equiv.) using set-up 4 at $60{ }^{\circ} \mathrm{C}$ (Figure S7). The crude mixture was purified by flash column chromatography on silica gel (30\% EtOAc in hexane as eluent) to afford $11 \mathrm{n}(56.2 \mathrm{mg}, 74 \%$ yield) as a white solid.

${ }^{1} \mathrm{H} \mathrm{NMR}\left(400 \mathrm{MHz}, \mathrm{CDCl}_{3}\right) \delta 8.04(\mathrm{dd}, J=8.5,1.3 \mathrm{~Hz}, 1 \mathrm{H}), 7.85(\mathrm{dd}, J=8.5,1.4 \mathrm{~Hz}, 1 \mathrm{H}), 7.74$ $-7.67(\mathrm{~m}, 2 \mathrm{H}), 7.63(\mathrm{ddd}, J=8.3,6.8,1.4 \mathrm{~Hz}, 1 \mathrm{H}), 7.43(\mathrm{ddd}, J=8.3,6.8,1.3 \mathrm{~Hz}, 1 \mathrm{H}), 7.36(\mathrm{~d}$, $J=2.0 \mathrm{~Hz}, 2 \mathrm{H}), 7.12(\mathrm{~s}, 1 \mathrm{H}), 4.08-3.96(\mathrm{~m}, 2 \mathrm{H}), 3.20(\mathrm{tt}, J=11.5,3.8 \mathrm{~Hz}, 1 \mathrm{H}), 2.71(\mathrm{~s}, 3 \mathrm{H})$, $2.47(\mathrm{~m}, 5 \mathrm{H}), 2.07-1.85(\mathrm{~m}, 4 \mathrm{H})$.

${ }^{13} \mathrm{C} \mathrm{NMR}\left(101 \mathrm{MHz}, \mathrm{CDCl}_{3}\right) \delta 159.0,150.3,148.2,143.8,133.1,129.9,129.8,129.2,127.9$, $125.8,124.8,122.2,118.6,47.0,36.4,31.8,25.5,21.7$.

HRMS: calculated for $\mathrm{C}_{22} \mathrm{H}_{25} \mathrm{~N}_{2} \mathrm{O}_{2} \mathrm{~S}\left(\mathrm{M}+\mathrm{H}^{+}\right)$: 381.1627, found 381.1631 (+1.1 ppm).

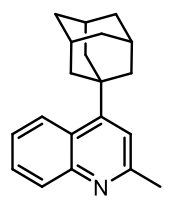

4-((3s)-Adamantan-1-yl)-2-methylquinoline (11o): Synthesized according to General Procedure J using 1,3-dioxoisoindolin-2-yl (3r,5r,7r)-adamantane-1carboxylate $\mathbf{1 h}(130 \mathrm{mg}, 0.4 \mathrm{mmol}, 2$ equiv. $)$ and 2-methylquinoline $\mathbf{1 0 b}(27.1 \mu \mathrm{L}$ , 0.2 mmol, 1.0 equiv.) using set-up 4 at $60{ }^{\circ} \mathrm{C}$ (Figure S7). The crude mixture was purified by flash column chromatography on silica gel (10\% EtOAc in hexane as eluent) to afford 11n (38.2 mg, 69\% yield) as a white solid.

${ }^{1} \mathrm{H}$ NMR $\left(400 \mathrm{MHz}, \mathrm{CDCl}_{3}\right) \delta 8.62(\mathrm{~d}, J=8.8 \mathrm{~Hz}, 1 \mathrm{H}), 8.35(\mathrm{~s}, 1 \mathrm{H}), 7.69(\mathrm{ddd}, J=8.3,6.8,1.3$ $\mathrm{Hz}, 1 \mathrm{H}), 7.52(\mathrm{ddd}, J=8.5,6.8,1.5 \mathrm{~Hz}, 1 \mathrm{H}), 7.28(\mathrm{~s}, 1 \mathrm{H}), 2.85(\mathrm{~s}, 3 \mathrm{H}), 2.29-2.28(\mathrm{~m}, 6 \mathrm{H}), 2.23$ - $2.22(\mathrm{~m}, 3 \mathrm{H}), 1.89(\mathrm{t}, J=3.1 \mathrm{~Hz}, 6 \mathrm{H})$.

${ }^{13} \mathrm{C} \mathrm{NMR}\left(101 \mathrm{MHz}, \mathrm{CDCl}_{3}\right) \delta 159.5,130.1,126.3,125.4,125.3,119.7,42.3,39.3,36.9,29.1$. HRMS: calculated for $\mathrm{C}_{20} \mathrm{H}_{24} \mathrm{~N}\left(\mathrm{M}+\mathrm{H}^{+}\right)$: 278.1897, found $278.1903(+2.2 \mathrm{ppm})$.

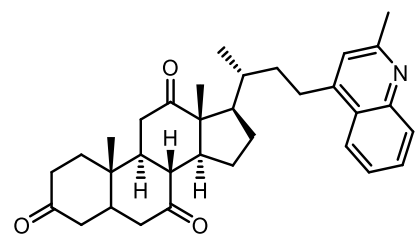

$(8 R, 9 S, 10 S, 13 R, 14 S, 17 R)-10,13-d i m e t h y l-17-((R)-4-(2-$ methylquinolin-4-yl)butan-2-yl)dodecahydro-3Hcyclopenta[a]phenanthrene-3,7,12(2H,4H)-trione (11p):

Synthesized according to General Procedure J using Dehydrocholic acid phthalimide ester $\mathbf{1 d}(219 \mathrm{mg}, 0.4 \mathrm{mmol}, 2.0$ equiv.) and 2methylquinoline $\mathbf{1 0 b}(27.1 \mu \mathrm{L}, 0.2 \mathrm{mmol}, 1.0$ equiv. $)$. The crude mixture was purified by flash column chromatography on silica gel (30\% EtOAc in hexane as eluent) to afford $11 p$ ( $70.0 \mathrm{mg}, 61 \%$ yield) as a white solid.

${ }^{1} \mathrm{H} \mathrm{NMR}\left(400 \mathrm{MHz}, \mathrm{CDCl}_{3}\right) \delta 8.12(\mathrm{~d}, J=8.4 \mathrm{~Hz}, 1 \mathrm{H}), 7.96(\mathrm{dd}, J=8.5,1.4 \mathrm{~Hz}, 1 \mathrm{H}), 7.67(\mathrm{ddd}$, $J=8.4,6.9,1.4 \mathrm{~Hz}, 1 \mathrm{H}), 7.51(\mathrm{ddd}, J=8.2,6.9,1.3 \mathrm{~Hz}, 1 \mathrm{H}), 7.14(\mathrm{~s}, 1 \mathrm{H}), 3.15(\mathrm{ddd}, J=13.7$, 11.7, 5.0 Hz, 1H), $2.96-2.85(\mathrm{~m}, 4 \mathrm{H}), 2.74(\mathrm{~s}, 3 \mathrm{H}), 2.41-1.22(\mathrm{~m}, 19 \mathrm{H}), 1.39(\mathrm{~s}, 3 \mathrm{H}), 1.08(\mathrm{~s}$, $3 \mathrm{H}), 1.05(\mathrm{~d}, J=6.6 \mathrm{~Hz}, 3 \mathrm{H})$.

${ }^{13} \mathrm{C} \mathrm{NMR}\left(101 \mathrm{MHz}, \mathrm{CDCl}_{3}\right) \delta 212.1,209.1,208.8,158.4,150.6,146.9,129.7,128.6,126.0$, 125.9, 123.4, 121.8, 57.0, 51.9, 49.1, 46.9, 45.7, 45.6, 45.1, 42.9, 38.7, 36.6, 36.5, 36.3, 36.1, $35.4,29.5,27.9,25.2,24.8,22.0,19.2,12.0$.

HRMS: calculated for $\mathrm{C}_{33} \mathrm{H}_{42} \mathrm{NO}_{3}\left(\mathrm{M}+\mathrm{H}^{+}\right)$: 500.3157, found: 500.3159 (+0.4 ppm). 


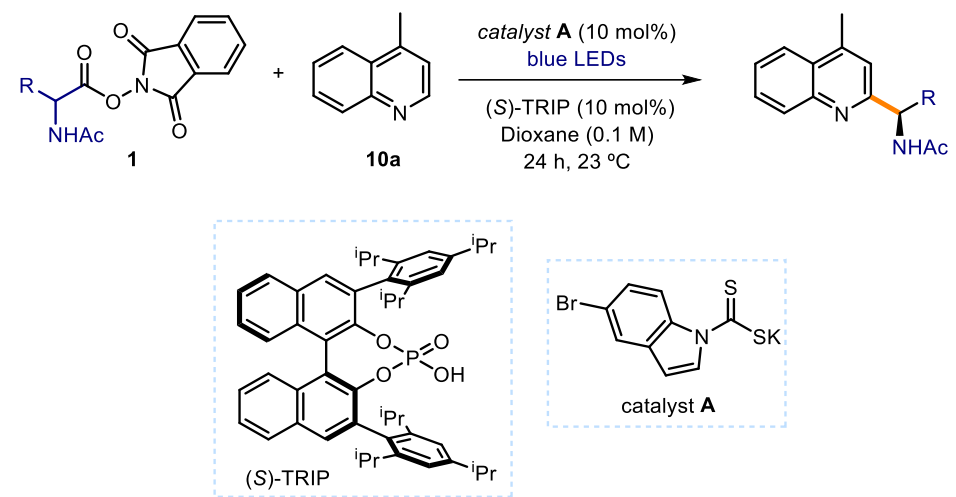

Reactions performed using set-up 4 in Figure S7. In an oven dried vial, with a Teflon septum screw cap, 4-methylquinoline $(26.4 \mu \mathrm{L}, 0.2 \mathrm{mmol}, 1.0$ equiv.) and chiral phosphoric acid $(S)$ TRIP ( $7.5 \mathrm{mg}, 0.01 \mathrm{mmol}, 5 \mathrm{~mol} \%)$ were dissolved in dioxane $(2 \mathrm{ml})$. Then, phthalimide ester 1 ( $0.4 \mathrm{mmol}, 2$ equiv. $)$ and catalyst $\mathbf{A}(6.2 \mathrm{mg}, 0.02 \mathrm{mmol}, 0.1$ equiv.) were added. The resulting yellow mixture was degassed with argon sparging for 60 seconds. The vial was placed in the oneposition photoreactor with temperature control (Figure S7) and irradiated under stirring for 24 hours, unless otherwise specified. The solvent was evaporated, and the residue purified by column chromatography to afford the corresponding product in the stated yield with $>95 \%$ purity according to ${ }^{1} \mathrm{H}$ NMR analysis.

\section{C5.4 Characterization of Products}

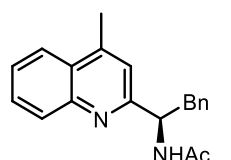

(R)- $N$-(1-(4-methylquinolin-2-yl)-2-phenylethyl) acetamide (11q): Synthesized according to General Procedure K using 1,3-dioxoisoindolin-2-yl acetylphenylalaninate $\mathbf{1 i}(140 \mathrm{mg}, 0.4 \mathrm{mmol})$ and 4-methylquinoline 10a (26.4 $\mu \mathrm{L}, 0.2 \mathrm{mmol})$. The crude mixture was purified by flash column chromatography on silica gel (40\% EtOAc in hexane as eluent) to afford 11q $(60.0 \mathrm{mg}, 97 \%$ yield) as a white solid.

${ }^{1} \mathrm{H} \mathrm{NMR}\left(400 \mathrm{MHz}, \mathrm{CDCl}_{3}\right) \delta 8.05(\mathrm{dt}, J=8.5,0.9 \mathrm{~Hz}, 1 \mathrm{H}), 7.96(\mathrm{ddd}, J=8.3,1.5,0.6 \mathrm{~Hz}, 1 \mathrm{H})$, 7.70 (ddd, $J=8.4,6.9,1.5 \mathrm{~Hz}, 1 \mathrm{H}), 7.59-7.51(\mathrm{~m}, 1 \mathrm{H}), 7.27(\mathrm{~s}, 1 \mathrm{H}), 7.18-7.13(\mathrm{~m}, 3 \mathrm{H}), 6.99$ $-6.92(\mathrm{~m}, 2 \mathrm{H}), 6.82(\mathrm{~d}, J=1.2 \mathrm{~Hz}, 1 \mathrm{H}), 5.39(\mathrm{td}, J=7.7,5.4 \mathrm{~Hz}, 1 \mathrm{H}), 3.35(\mathrm{dd}, J=13.3,5.4 \mathrm{~Hz}$, $1 \mathrm{H}), 3.17(\mathrm{dd}, J=13.3,7.9 \mathrm{~Hz}, 1 \mathrm{H}), 2.59(\mathrm{~d}, J=0.9 \mathrm{~Hz}, 3 \mathrm{H}), 2.07$ (s, $3 \mathrm{H})$.

${ }^{13} \mathrm{C}$ NMR $\left(101 \mathrm{MHz}, \mathrm{CDCl}_{3}\right) \delta 169.6,158.9,147.1,144.9,137.3,129.8,129.5,129.4,128.2$, 127.6, 126.6, 126.3, 124.00, 121.7, 55.6, 42.3, 23.7, 18.9.

Matching reported literature data. ${ }^{4}$<smiles>Cc1cc(C(C)N)nc2ccccc12</smiles>

(R)-N-(1-(4-methylquinolin-2-yl) ethyl) acetamide (11r): Synthesized according to General Procedure K using 1,3-dioxoisoindolin-2-yl acetylalaninate $\mathbf{1 j}(110 \mathrm{mg}, 0.4 \mathrm{mmol})$ and 4 -methylquinoline 10a $(26.4 \mu \mathrm{L}, 0.2 \mathrm{mmol})$. The crude mixture was purified by flash column chromatography on silica gel $(40 \%$ AcOEt in hexane as eluent) to afford $\mathbf{1 1} \mathbf{r}(38.3 \mathrm{mg}, 84 \%$ yield) as a white solid.

${ }^{1} \mathrm{H}$ NMR $\left(400 \mathrm{MHz}, \mathrm{CDCl}_{3}\right) \delta 8.08(\mathrm{dt}, J=8.1,1.0 \mathrm{~Hz}, 1 \mathrm{H}), 7.98(\mathrm{ddd}, J=8.3,1.5,0.7 \mathrm{~Hz}, 1 \mathrm{H})$, 7.71 (ddd, $J=8.4,6.9,1.4 \mathrm{~Hz}, 1 \mathrm{H}), 7.56$ (ddd, $J=8.3,6.9,1.3 \mathrm{~Hz}, 1 \mathrm{H}), 7.51$ (s, $1 \mathrm{H}), 7.17$ (d, $J$ $=1.1 \mathrm{~Hz}, 1 \mathrm{H}), 5.21(\mathrm{p}, J=6.8 \mathrm{~Hz}, 1 \mathrm{H}), 2.70(\mathrm{~d}, J=1.0 \mathrm{~Hz}, 3 \mathrm{H}), 2.11(\mathrm{~s}, 3 \mathrm{H}), 1.54(\mathrm{~d}, J=6.8 \mathrm{~Hz}$, $3 \mathrm{H})$.

${ }^{13} \mathrm{C} \mathrm{NMR}\left(101 \mathrm{MHz}, \mathrm{CDCl}_{3}\right) \delta 169.6,160.7,146.9,145.8,129.6,129.3,127.6,126.4,123.9$, 120.5, 50.1, 23.74, 22.8, 19.0.

Matching reported literature data. ${ }^{4}$ 


\section{C6 Trifluoromethylation}

C6.1 Procedure

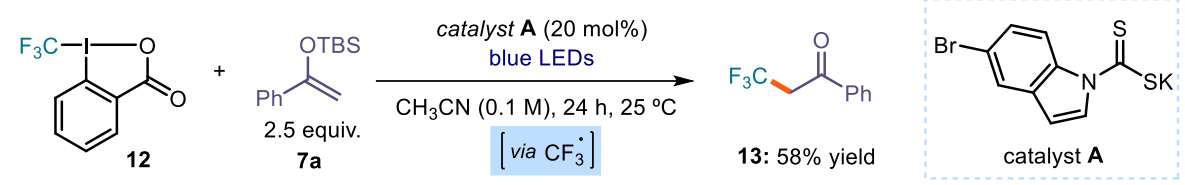

Reactions performed using set-up 3 in Figure S6. In an oven dried vial with a Teflon septum screw cap, silyl enol ether 7a (117 mg, $0.5 \mathrm{mmol}, 2.5$ equiv.) was dissolved in $\mathrm{MeCN}(2 \mathrm{~mL})$, then the Togni reagent $12(105 \mathrm{mg}(60 \% \mathrm{Wt}) 0.2 \mathrm{mmol}, 1.0$ equiv.), catalyst $\mathbf{A}(6.2 \mathrm{mg}, 0.02$ mmol, 0.1 equiv.). The resulting orange mixture was degassed bubbling argon for 60 seconds. The vial was then placed in the irradiation setup (see Figure S6), maintained at a temperature of $25{ }^{\circ} \mathrm{C}\left(25-26{ }^{\circ} \mathrm{C}\right.$ measured in the central well), and the reaction, unless otherwise stated, was stirred for 24 hours under continuous irradiation from a blue LED strip. The crude mixture was diluted with AcOEt and brine was added. The combined organic fractions were dried over anhydrous $\mathrm{MgSO}_{4}$, filtered, and concentrated to dryness. The crude mixture was purified by flash column chromatography on silica gel (Hexane/AcOEt) to afford $\mathbf{1 3}(22 \mathrm{mg}, 58 \%$ yield) as a yellow oil.

${ }^{1} \mathrm{H} \mathrm{NMR}\left(300 \mathrm{MHz}, \mathrm{CDCl}_{3}\right) \delta 8.01-7.91(\mathrm{~m}, 2 \mathrm{H}), 7.69-7.61(\mathrm{~m}, 1 \mathrm{H}), 7.60-7.46(\mathrm{~m}, 3 \mathrm{H})$, $3.82(\mathrm{q}, J=10.0 \mathrm{~Hz}, 2 \mathrm{H})$.

${ }^{19} \mathrm{~F} \mathrm{NMR}\left(376 \mathrm{MHz}, \mathrm{CDCl}_{3}\right) \delta-62.11$.

${ }^{13} \mathrm{C} \mathrm{NMR}(126 \mathrm{MHz}, \mathrm{CDCl} 3) \delta 135.7,134.2,128.9,128.4,124.7,42.2$.

Matching reported literature data. ${ }^{31}$

\section{C7 Amidyl Radical Cyclization}

\section{C7.1 Procedure}<smiles>C=CCCC(=O)N(Oc1ccc([N+](=O)[O-])cc1[N+](=O)[O-])[N+](=O)[O-]</smiles>
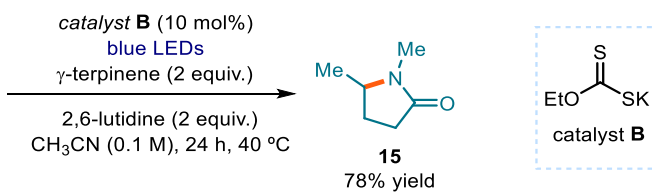

Reactions performed using set-up 1 in Figure S2. In an oven dried vial, with a Teflon septum screw cap, 14 (29 mg, $0.1 \mathrm{mmol}, 1$ equiv.) and catalyst $\mathbf{B}$ (1.6 mg, $0.01 \mathrm{mmol}, 0.1$ equiv.) were dissolved in $\mathrm{CH}_{3} \mathrm{CN}(1 \mathrm{~mL})$, then 2,6-lutidine ( $23 \mu \mathrm{L}, 0.2 \mathrm{mmol}, 2.0$ equiv.) was added, followed by $\gamma$-terpinene ( $32 \mu \mathrm{L}, 0.2 \mathrm{mmol}, 2$ equiv.). The resulting orange mixture was degassed with argon sparging for 60 seconds. The vial was then placed in the 3D printed support photoreactor (Figure S2) and irradiated under stirring for 16 hours. The crude mixture was concentrated to dryness. Trichloroethylene was added as internal standard $(9 \mu \mathrm{L}, 0.1 \mathrm{mmol}, 1.0$ equiv) and NMR yield was determined by ${ }^{1} \mathrm{H} \mathrm{NMR}$ in $\mathrm{CDCl}_{3}$ matching reported literature data. ${ }^{14}$ 


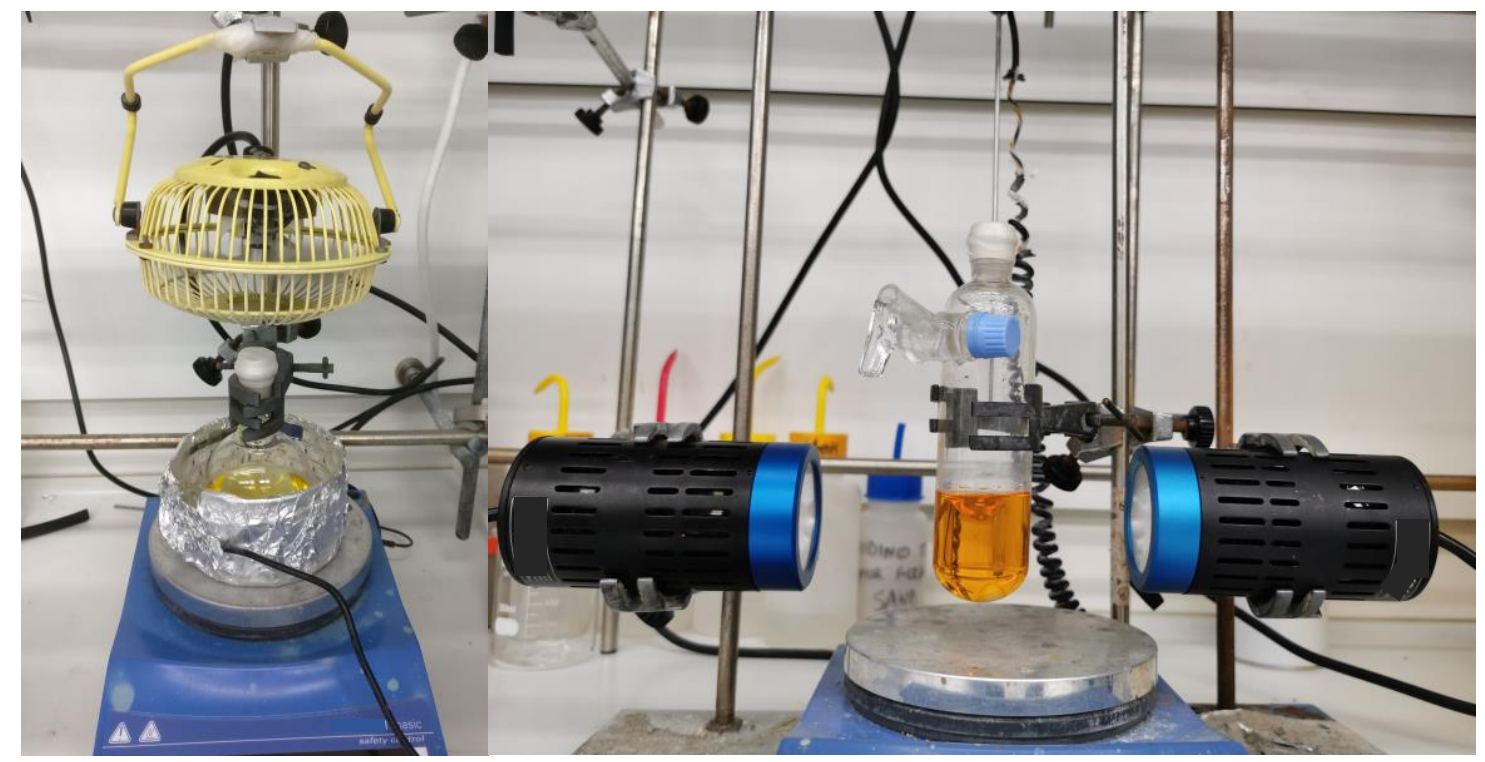

Figure S8: Experimental setup used for the large scale set up. (Left) Barton decarboxylation. (Right) Minisci reaction.

\section{C8.1 Barton decarboxylation}

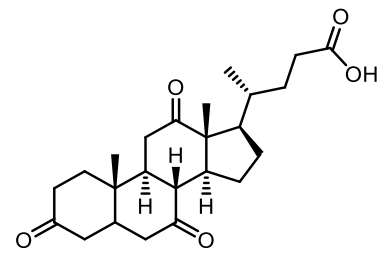

dehydrocholic acid

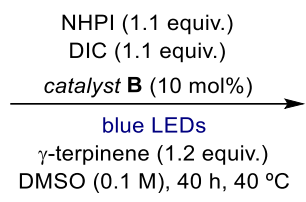

DMSO (0.1 M), $40 \mathrm{~h}, 40^{\circ} \mathrm{C}$

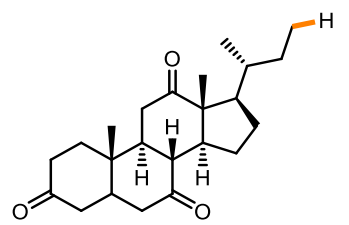

6d: $55 \%$ yield $(4 \mathrm{mmol})$

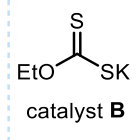

Using set-up in Figure S8. In a $100 \mathrm{~mL}$ round bottom flask with a Teflon cap, dehydrocholic acid (1,62 g, 4 mmol, 1 equiv.), $N$-hydroxyphthalimide (NHPI, $716 \mathrm{mg}, 4.4 \mathrm{mmol}, 1.1$ equiv.), and xanthogenate catalyst A (64 mg, $0.4 \mathrm{mmol}, 0.1$ equiv.) were dissolved in DMSO (40 mL), and $N, N^{\prime}$-diisopropylcarbodiimide (DIC, $760 \mu \mathrm{L}, 4.4 \mathrm{mmol}, 1.1$ equiv.) was added via syringe. Then, $\gamma$-terpinene $(760 \mu \mathrm{L}, 4.8 \mathrm{mmol}, 1.2$ equiv.) was added. The resulting orange mixture was degassed with nitrogen sparging for $5 \mathrm{~min}$. The round bottom flask was then irradiated for 40 hours with a 1-meter $14 \mathrm{~W}$ blue LED strip and cooled with a fan to keep the temperature between 30 and $35^{\circ} \mathrm{C}$ (see

Figure S8, left). The mixture was transferred to an extraction funnel, $\mathrm{NaOH} 1 \mathrm{M}$ solution was added and the organic layer was extracted with $\mathrm{CH}_{2} \mathrm{Cl}_{2}$. The organic layer was dried over anhydrous $\mathrm{MgSO}_{4}$, filtered, and concentrated to dryness. The crude residue was purified by chromatography on silica gel (10\% AcOEt in hexanes) to afford $800 \mathrm{mg}$ of product $6 \mathbf{d}$ ( $2.2 \mathrm{mmol}$, $55 \%$ yield) as an off white solid. NMR analysis was consistent with product synthesized in the small scale process. 


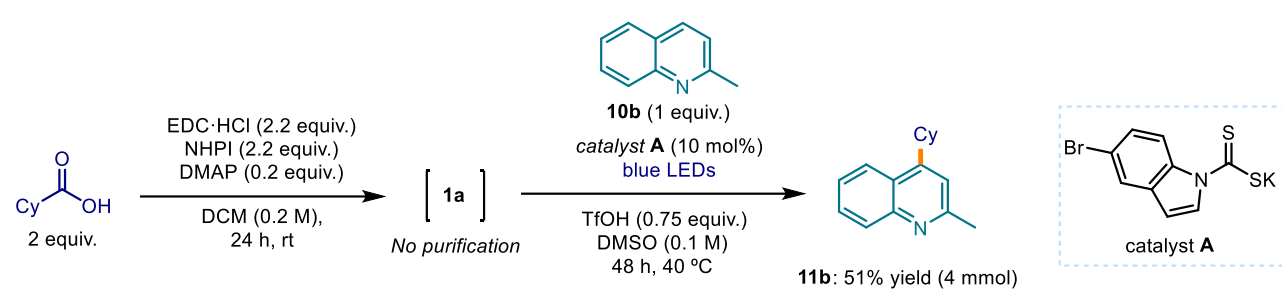

Using set-up in Figure $S 8$. In a $100 \mathrm{~mL}$ round bottom flask, cyclohexanecarboxylic acid (1.03 g, $8 \mathrm{mmol}, 2$ equiv.), EDC. $\mathrm{HCl}$ (1.69 g, $8.8 \mathrm{mmol}, 2.2$ equiv.), DMAP (97.7 mg, $0.8 \mathrm{mmol}, 0.2$ equiv.), and $\mathrm{N}$-hydroxyphthalimide (1.44 g, $8.8 \mathrm{mmol}, 2.2$ equiv.) were dissolved in $\mathrm{CH}_{2} \mathrm{Cl}_{2}(20$ $\mathrm{mL}$ ). The reaction was stirred at ambient temperature for 24 hours. The mixture was transferred to an extraction funnel, $\mathrm{NaHCO}_{3}$ sat. solution was added and the organic layer was extracted with $\mathrm{CH}_{2} \mathrm{Cl}_{2}$. The organic phase was concentrated to dryness under vacuum to obtain the crude phthalimide ester, which was used without further purification in the next step.

In a $100 \mathrm{~mL}$ Schlenk flask with a Teflon septum, the crude phthalimide ester was dissolved in DMSO $(40 \mathrm{~mL})$. Then, 2-methylquinoline $(540 \mu \mathrm{L}, 4.00 \mathrm{mmol}, 1.0$ equiv.), trifluoromethanesulfonic acid $(265 \mu \mathrm{L}, 3.00 \mathrm{mmol}, 0.75$ equiv.) and catalyst $\mathbf{A}$ (124 mg, 0.40 mmol, 0.1 equiv.) were added. The resulting orange mixture was degassed with nitrogen sparging for 5 minutes. The Schlenk flask was irradiated with stirring for 48 hours using two $50 \mathrm{~W}$ Kessil blue LED lamp (one PR160L-456 and one PR160L-427, 100\% intensity, 4-5 cm away) (see

Figure $\mathrm{S} 8$, right). The mixture was transferred to an extraction funnel, $\mathrm{NaHCO}_{3}$ sat. solution was added and the organic layer was extracted with $\mathrm{CH}_{2} \mathrm{Cl}_{2}$. The organic layer was dried over anhydrous $\mathrm{MgSO}_{4}$, filtered, and concentrated to dryness. The crude residue was purified by chromatography on silica gel (10\% AcOEt in hexanes) to afford $459 \mathrm{mg}$ of product 11b (2.04 mmol, $51 \%$ yield) as a yellowish oil. NMR analysis was consistent with product synthesized in the small scale process.

\section{C9. Unsuccessful Substrates}

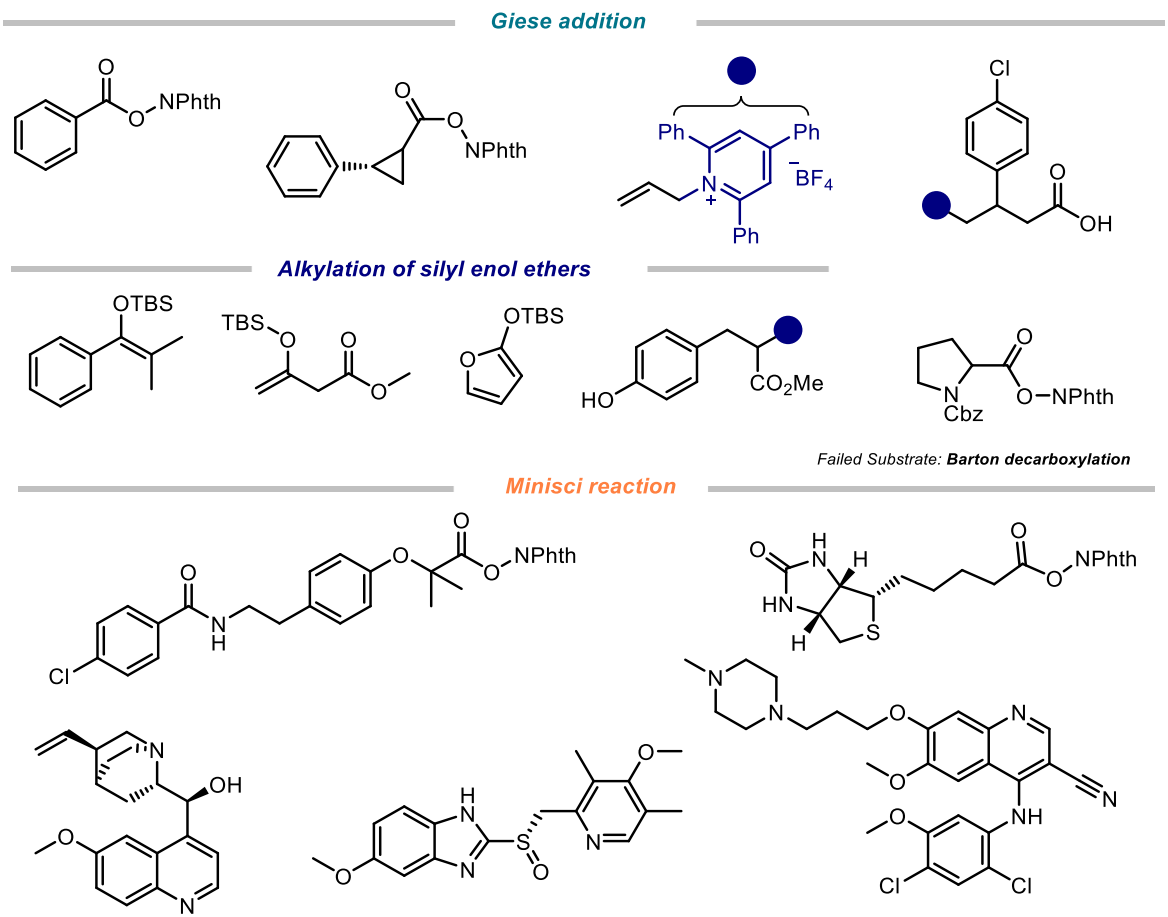

Figure S9: Unsuccessful substrates that offered poor yields (ranging from 0 to $<20 \%$ ) 


\section{Mechanistic Studies}

\section{D1. Control reactions}

\section{D1.1 Experiments with green light}

For the reactions performed under green light irradiation, an EvoluChem ${ }^{\mathrm{TM}}$ P303-30-1 LEDs (18 $\mathrm{W}, \lambda_{\max }=520 \mathrm{~nm}$ ) was used. The reaction temperature was measured to be between $25^{\circ} \mathrm{C}$ and 30 ${ }^{\circ} \mathrm{C}$ using the setup depicted in Figure S10).
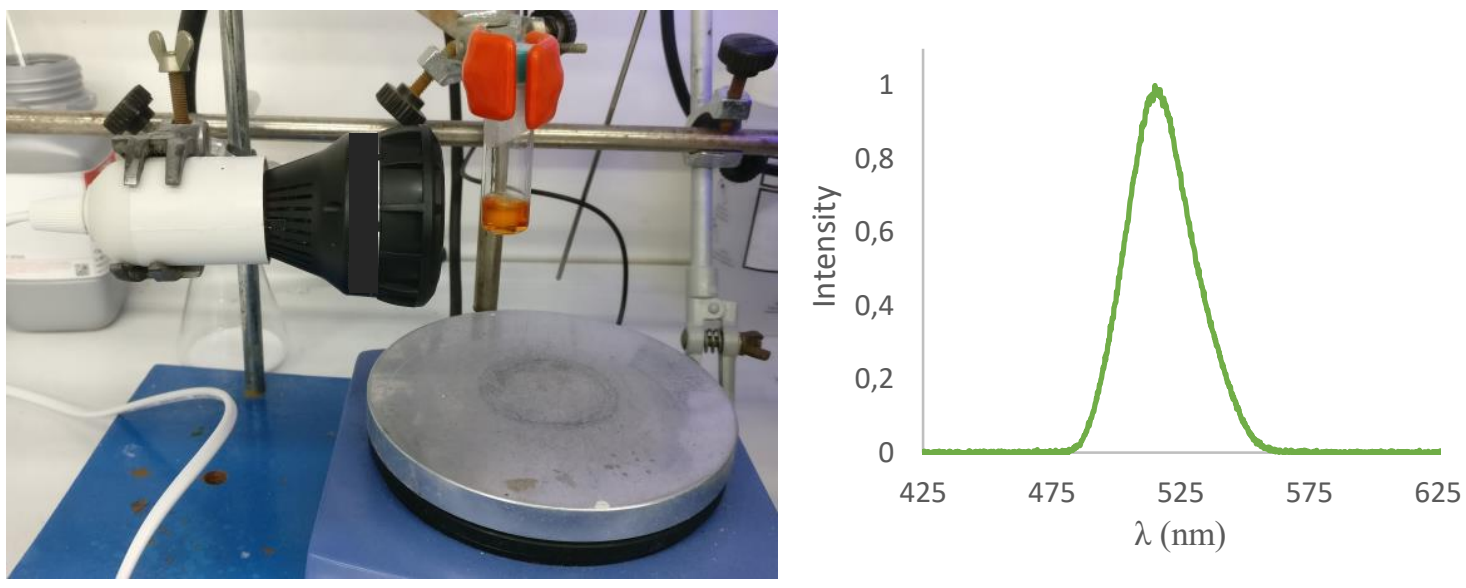

Figure S10: Reaction set-up for green light irradiation.

\section{D1.2 Optimization studies.}

Table S1. Giese addition with pyridinium salts as radical precursors

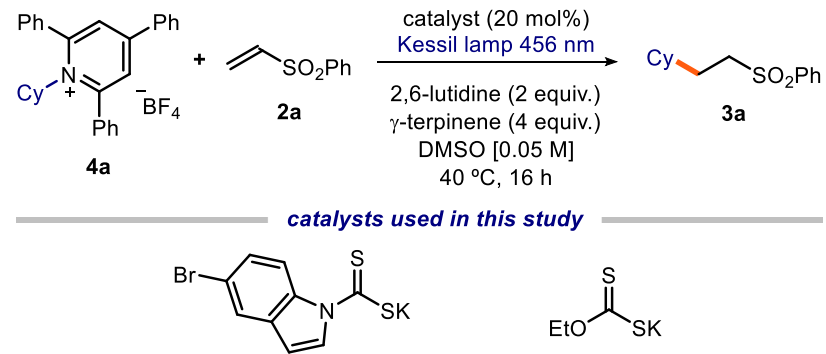

catalyst A catalyst B

\begin{tabular}{cccc}
\hline entry & catalyst & deviation & yield $(\%)^{\mathrm{a}}$ \\
\hline 1 & A & none & $83(67)^{\mathrm{b}}$ \\
2 & B & none & 70 \\
3 & A & Green light & 24 \\
4 & B & Green light & 20 \\
5 & A & under air & 0 \\
6 & A & no light & 0
\end{tabular}

Reactions performed using set-up 2 in Figure S3. All reactions performed under inert atmosphere on a $0.1 \mathrm{mmol}$ scale. ${ }^{a}$ Yield determined by ${ }^{1} \mathrm{H}$ NMR analysis of the crude mixture using trimethoxybenzene as the internal standard. ${ }^{\mathrm{b}}$ Yield of the isolated product on a $0.2 \mathrm{mmol}$ scale. 
Table S2. Barton decarboxylation

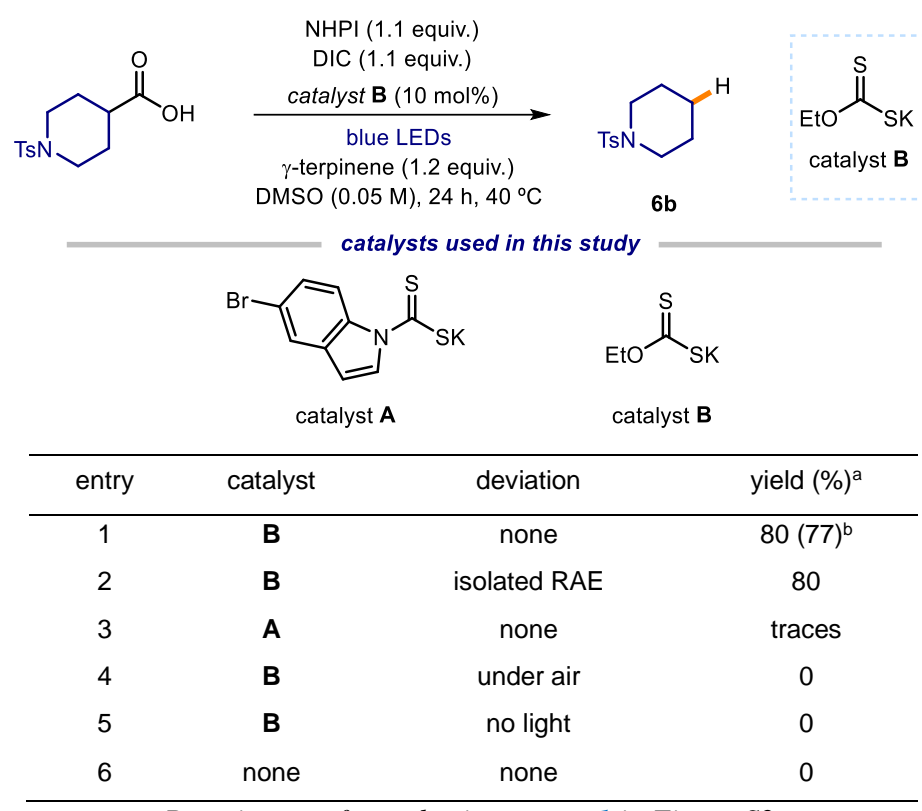

Reactions performed using set-up 1 in Figure S2.

Table S3. Deamination with pyridinium salts as radical precursors

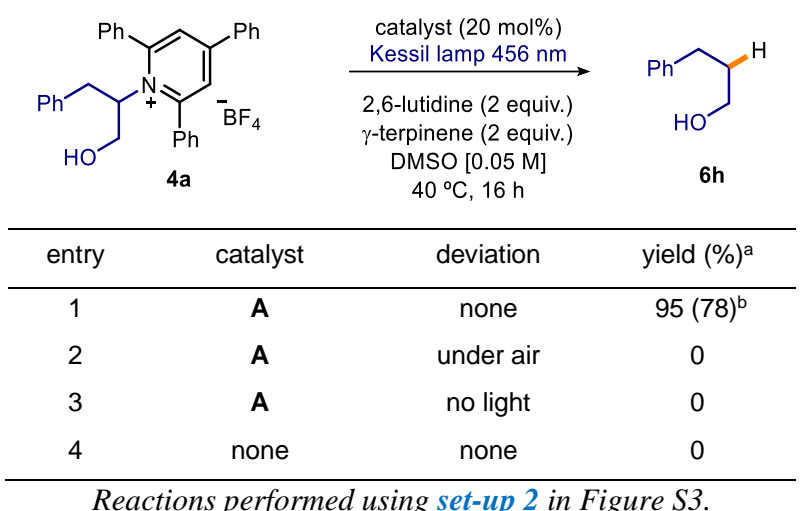


Table S4. $\alpha$-alkylation of silyl enol ethers

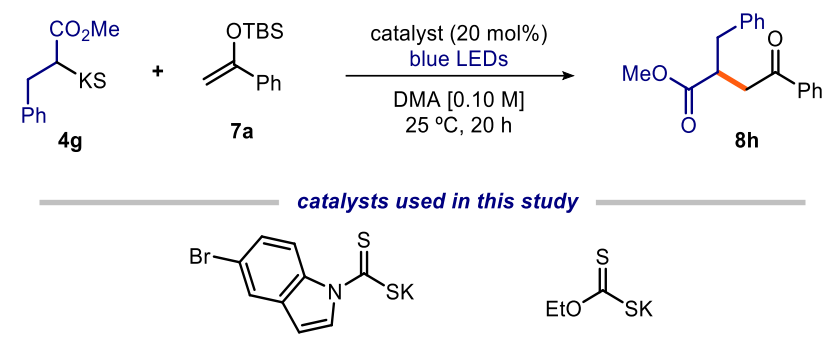

catalyst $\mathbf{A}$

catalyst B

\begin{tabular}{ccccc}
\hline entry & catalyst & Base & deviation & yield (\%) $^{\mathrm{a}}$ \\
\hline 1 & A & - & none & 16 \\
2 & A & Lutidine (1 equiv.) & none & $95(89 \%)^{\mathrm{b}}$ \\
3 & B & Lutidine (1 equiv.) & none & 80 \\
4 & B & Lutidine (1 equiv.) & Green light & 45 \\
5 & A & Lutidine (1 equiv.) & cat. 10 mol\% & 62 \\
6 & A & Lutidine (1 equiv.) & under air & 0 \\
7 & A & Lutidine (1 equiv.) & no light & 0 \\
8 & none & Lutidine (1 equiv.) & none & 0 \\
\hline \multicolumn{5}{c}{ Reactions performed using set-up 3 in Figure S6. }
\end{tabular}

Table S5. Minisci reaction

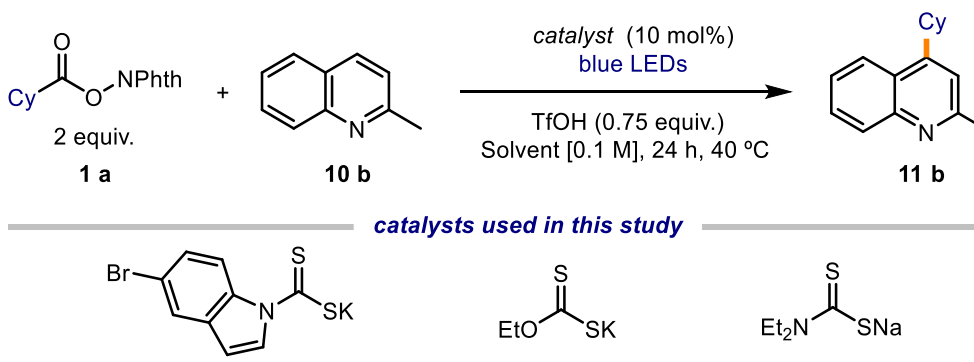

\begin{tabular}{ccccc} 
& catalyst A & \multicolumn{2}{c}{ catalyst B } & \multicolumn{2}{c}{ catalyst C } \\
\hline entry & catalyst & Solvents & deviation & yield (\%) \\
\hline 1 & A & DMSO & TFA & 82 \\
2 & B & DMSO & TFA & 0 \\
3 & C & DMSO & TFA & 0 \\
4 & A & DMA & TFA & 41 \\
5 & A & NMP & TFA & 57 \\
6 & A & DMSO & none & $97(95)^{\mathrm{b}}$ \\
7 & A & DMSO & Cat. 5 mol\% & 64 \\
8 & A & DMSO & Green light & 8 \\
9 & A & DMSO & No acid & 0 \\
10 & A & DMSO & no light & 0 \\
11 & A & DMSO & under air & 0 \\
12 & none & DMSO & none & 0 \\
\hline
\end{tabular}

Reactions performed using set-up 2 in Figure S3. 
Table S6. Trifluoromethylation of silyl enol ethers.

catalyst $\mathbf{A}(20 \mathrm{~mol} \%)$
blue LEDs

Table S7 Minisci reaction

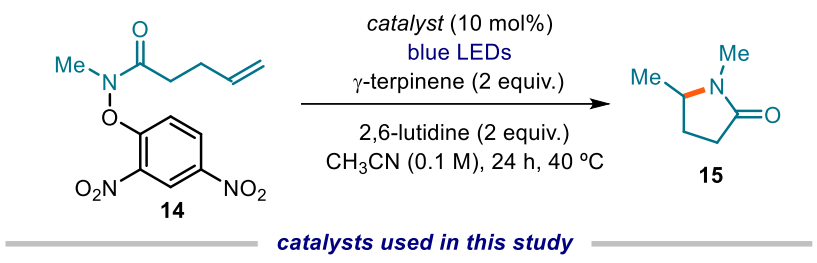

\begin{tabular}{|c|c|c|c|}
\hline & catalyst A & cataly & \\
\hline entry & catalyst & deviation & yield $(\%)^{a}$ \\
\hline 1 & A & None & 86 \\
\hline 2 & B & None & 78 \\
\hline 3 & A & No base & 60 \\
\hline 4 & B & No base & 50 \\
\hline 5 & A & Under air & 0 \\
\hline 6 & A & No light & 0 \\
\hline 7 & none & None & 0 \\
\hline
\end{tabular}

Reactions performed using set-up 1 in Figure S2.

D1.3 TEMPO Trapping Experiment.
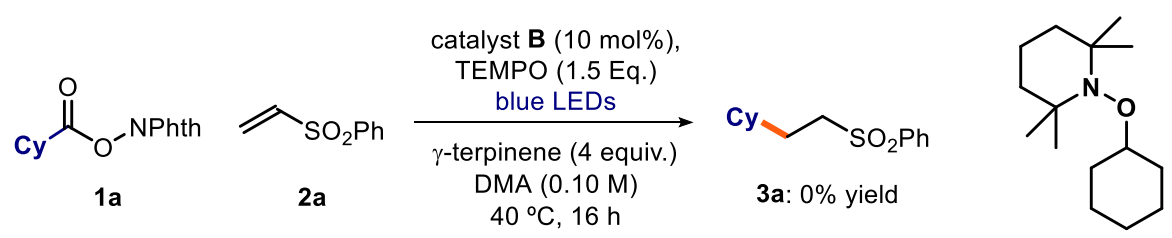

Cy-TEMPO

$52 \%$ yield

Reactions performed using set-up 1 in Figure S2. In an oven dried vial with a Teflon septum screw cap, potassium ethyl xanthogenate B (1.6 mg, $0.01 \mathrm{mmol}, 0.1$ equiv.), phthalimide ester 1a (0.1 mmol, $27.3 \mathrm{mg}, 1$ equiv.), 2,2,6,6-tetramethylpiperidine 1-oxyl (TEMPO, $0.15 \mathrm{mmol}, 23.5$ 
$\mathrm{mg}, 1.5$ equiv. $)$ and $\mathbf{2 a}(0.15 \mathrm{mmol}, 25.2 \mathrm{mg}, 1.5$ equiv. $)$ were dissolved in DMA ( $1 \mathrm{~mL})$. Then, $\gamma$-terpinene ( $64 \mu \mathrm{L}, 0.4 \mathrm{mmol}, 4$ equiv.) was added. The resulting orange mixture was degassed by argon sparging for 60 seconds. The vial was then placed in the 3D printed support photoreactor (Figure S2) and irradiated under stirring for 16 hours. The mixture was transferred to an extraction funnel, brine was added and the organic layer was extracted with EtOAc. The organic layer was dried over anhydrous $\mathrm{MgSO}_{4}$, filtered, and concentrated to dryness. The crude residue was then purified by column chromatography (2\% EtOAc in hexanes) to afford the corresponding $\mathrm{Cy}-$ TEMPO adduct in $52 \%$ yield. No product corresponding with giese addition was detected in the crude NMR or during the purification.

${ }^{1} \mathrm{H}$ NMR $\left(500 \mathrm{MHz}, \mathrm{CDCl}_{3}\right) \delta 3.68-3.64(\mathrm{~m}, 1 \mathrm{H}), 2.07$ (brs, 2H), 1.76 (brs, 2H), $1.57-1.54$ $(\mathrm{m}, 2 \mathrm{H}), 1.48-1.45(\mathrm{~m}, 4 \mathrm{H}), 1.31-1.06(\mathrm{~m}, 19 \mathrm{H})$.

${ }^{13} \mathrm{C} \mathrm{NMR}\left(126 \mathrm{MHz}, \mathrm{CDCl}_{3}\right) \delta 81.7,59.6,40.3,32.9,26.0,25.1,17.3$.

Characterization data matching data reported in the literature. ${ }^{32}$

\section{D1.4 Catalysts' stability experiments}

- Catalyst A:
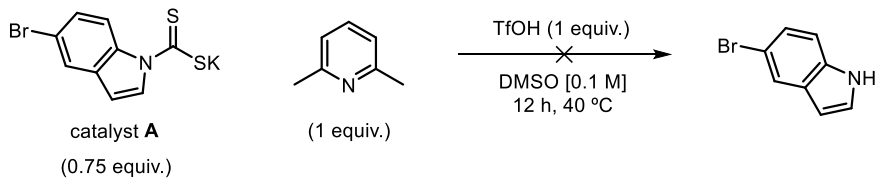

In an oven dried vial with a Teflon septum screw cap, 2,6-lutidine $(0.1 \mathrm{mmol}, 12 \mu \mathrm{L})$ was added followed by triflic acid $(0.1 \mathrm{mmol}, 8.9 \mu \mathrm{L})$. The resulting pyridinium salt was then dissolved in 1 $\mathrm{mL}$ of $\mathrm{d}^{6}$-DMSO, followed by the addition of catalyst $\mathbf{A}(23.3 \mathrm{mg}, 0.075 \mathrm{mmol})$. The mixture was stirred overnight and the crude mixture was analyzed by ${ }^{1} \mathrm{H}$ NMR. Catalyst $\mathbf{A}$ showed no degradation to the corresponding indole (see Fig. S11).

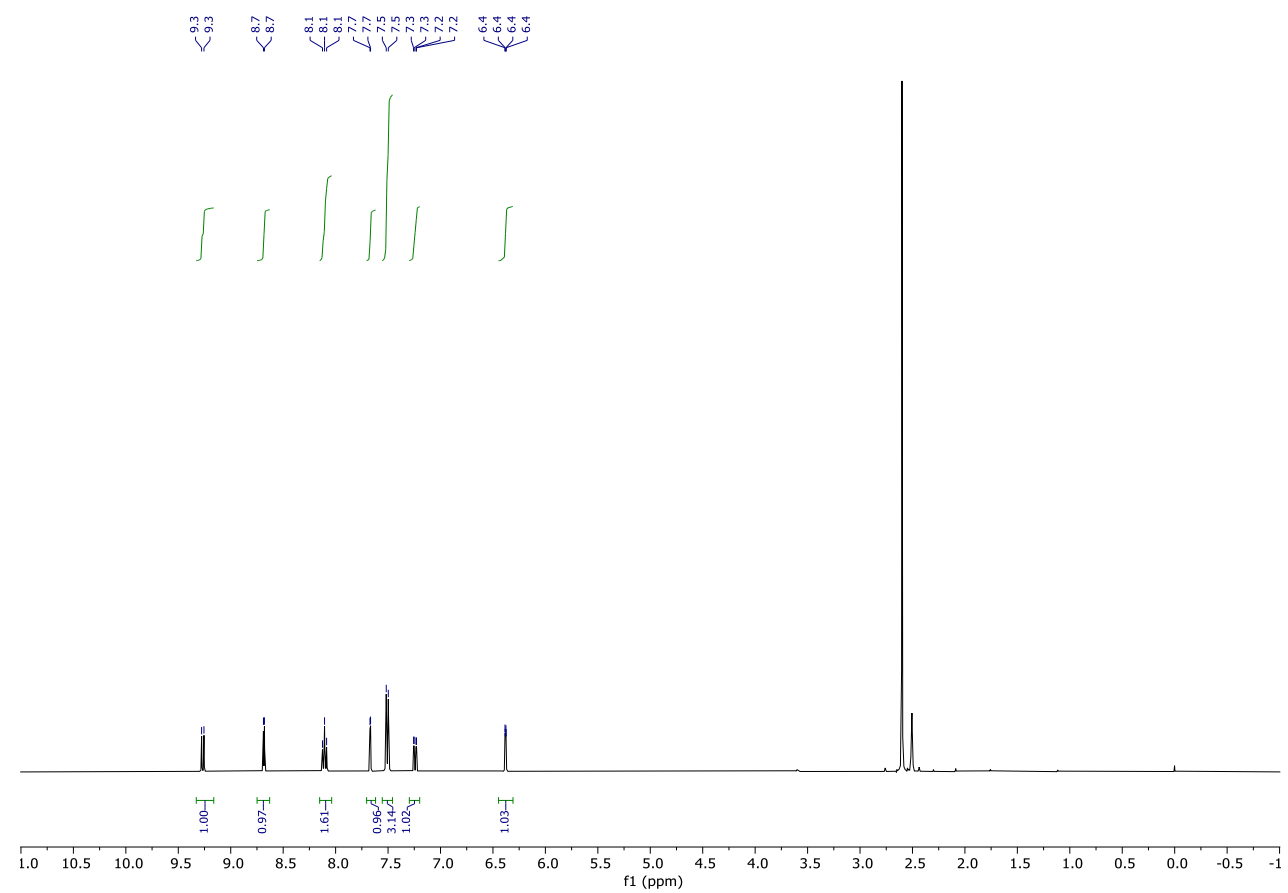

Figure S11. ${ }^{1} \mathrm{H}$ NMR analysis to evaluate catalyst A stability 
- Catalyst B:

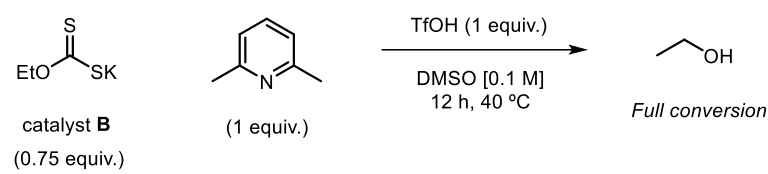

In an oven dried vial with a Teflon septum screw cap, 2,6-lutidine $(0.1 \mathrm{mmol}, 12 \mu \mathrm{L})$ was added followed by triflic acid $(0.1 \mathrm{mmol}, 8.9 \mu \mathrm{L})$. The resulting pyridinium salt was then dissolved in 1 $\mathrm{mL}$ of $\mathrm{d}^{6}$-DMSO, followed by the addition of catalyst $\mathbf{B}(12 \mathrm{mg}, 0.075 \mathrm{mmol})$. The mixture was left stirring overnight and the crude mixture is analyzed by ${ }^{1} \mathrm{H}$ NMR. Catalyst $\mathbf{B}$ showed complete degradation to ethanol (see Fig. S12).

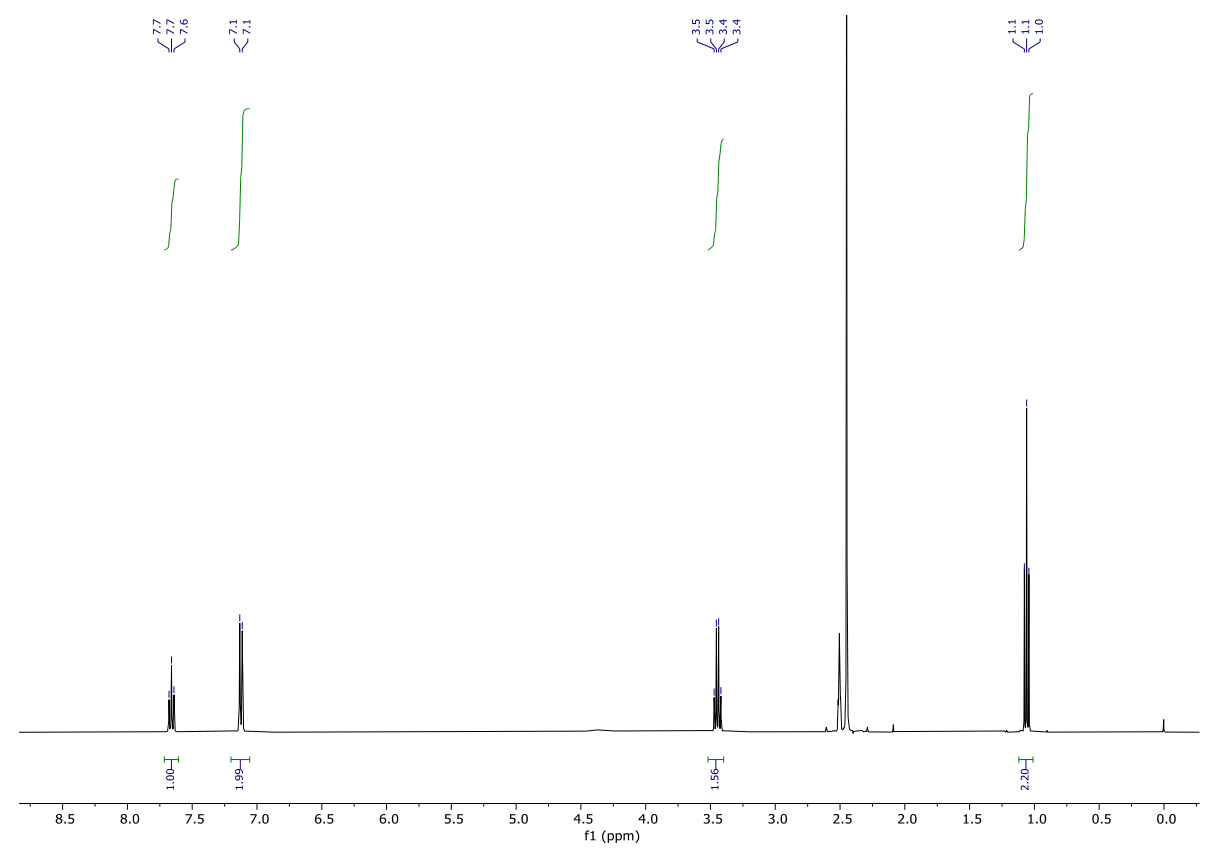

Figure S12. ${ }^{1} \mathrm{H}$ NMR analysis to evaluate catalyst $\mathbf{B}$ stability

\section{D2. UV-Vis measurements}

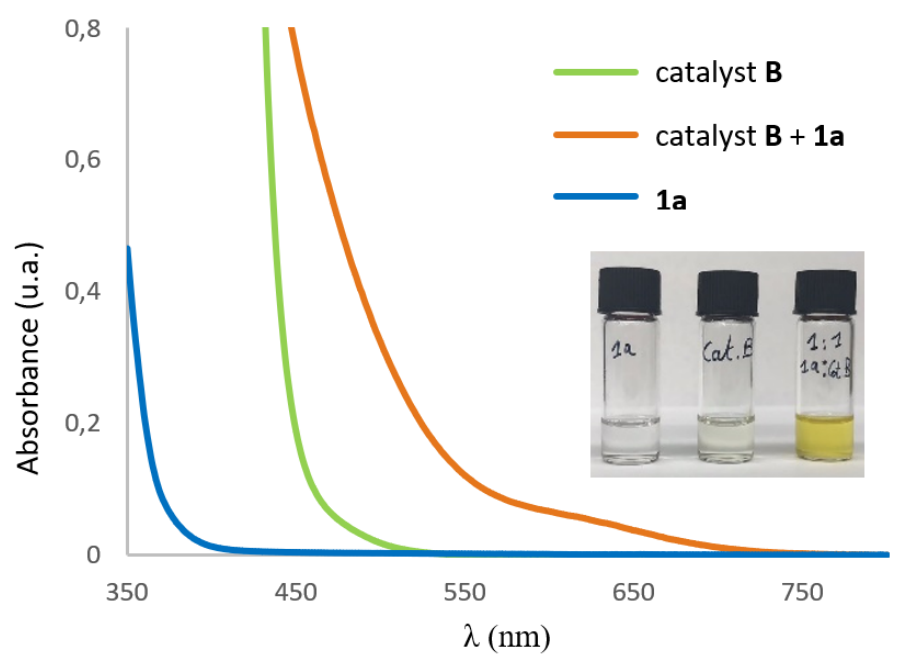

Figure S13: Optical absorption spectra, recorded in DMA in $1 \mathrm{~mm}$ path quartz cuvettes using a Shimadzu 2401PC UV-vis spectrophotometer, and visual appearance of the separate reaction components and of the colored EDA complex between catalyst $\mathbf{B}$ and $\mathbf{1 a}$. $[\mathbf{1 a}]=0.10 \mathrm{M}$, [ catalyst $\mathbf{B}]=0.01 \mathrm{M}$. 


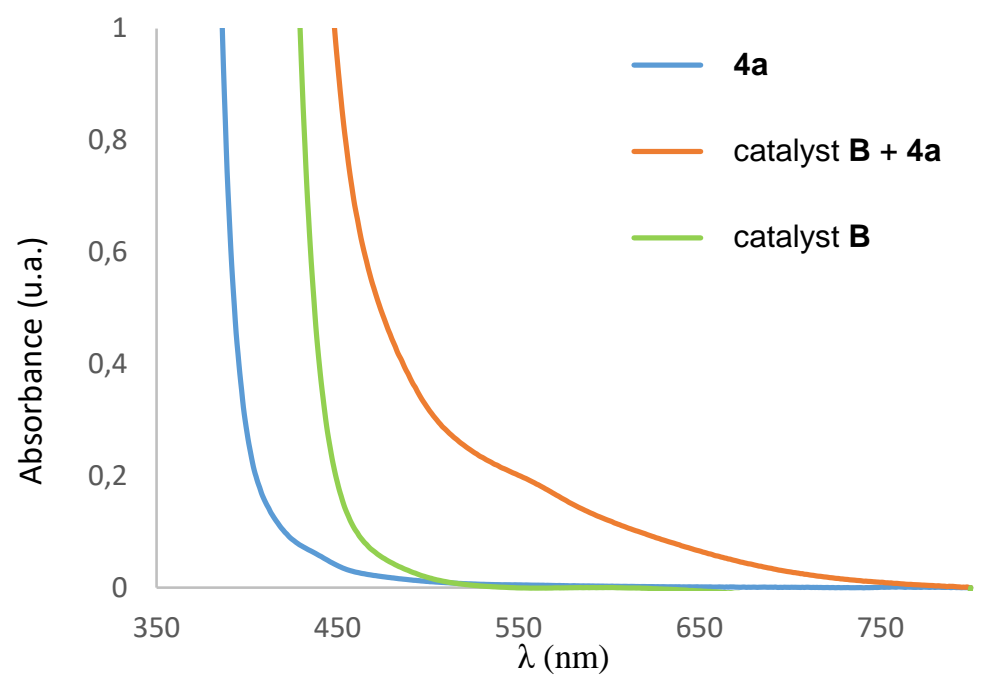

Figure S14: Optical absorption spectra, recorded in DMA in $1 \mathrm{~mm}$ path quartz cuvettes using a Shimadzu 2401PC UV-vis spectrophotometer of the separate reaction components and of the colored EDA complex between catalyst $\mathbf{B}$ and 4a. $[\mathbf{4 a}]=0.10 \mathrm{M}$, [catalyst $\mathbf{B}]=0.01 \mathrm{M}$.

\section{D3. Transient Absorption Spectroscopy (TAS).}

Studies with microsecond transient absorption spectroscopy (TAS) were performed using an excitation source of NdYAG (neodymium-doped yttrium aluminium garnet) Opolette laser with an optical parametric oscillator (OPO) system that allows variable wavelength excitation from $400-1800 \mathrm{~nm}$, pulse width of $6 \mathrm{~ns}$, up to $2 \mathrm{~mJ}$ of energy from OPO output with fiber optic coupled, and high energy output from direct NdYAG harmonics 355 (20 mJ, $5 \mathrm{~ns}$ ) and 532 (45mJ, $6 \mathrm{~ns}$ ). The system is completed with $150 \mathrm{~W}$ tungsten lamp as probe; 2 monochromators Minuteman MM151; Si amplified photodetector module for VIS; DSPDAU high speed data rate recorder and interface software from RAMDSP. Laser intensity for the chosen wavelength was $355 \mathrm{~nm}-1.30$ $\mathrm{mJ}$.

We selected a logarithmic time scale suitable for clearly showing the decay of the transient species in the samples. The characteristics of the detected transient species match literature data. ${ }^{33}$

In a typical transient absorption spectroscopy experiment, solutions in DMA of a mixture of 1a and catalyst $\mathbf{B}$ was prepared under an argon atmosphere and transferred into a screw-top $3.0 \mathrm{~mL}$ quartz cuvette for measurement. Upon irradiation with the appropriated wavelength, the decay of absorption at $620 \mathrm{~nm}$ of the transient xanthyl radical IIa was recorded.

Irradiation at $420 \mathrm{~nm}$ and $460 \mathrm{~nm}$ of the sample also provided signal absorbing at $620 \mathrm{~nm}$, but in a much lower intensity and higher noise. 


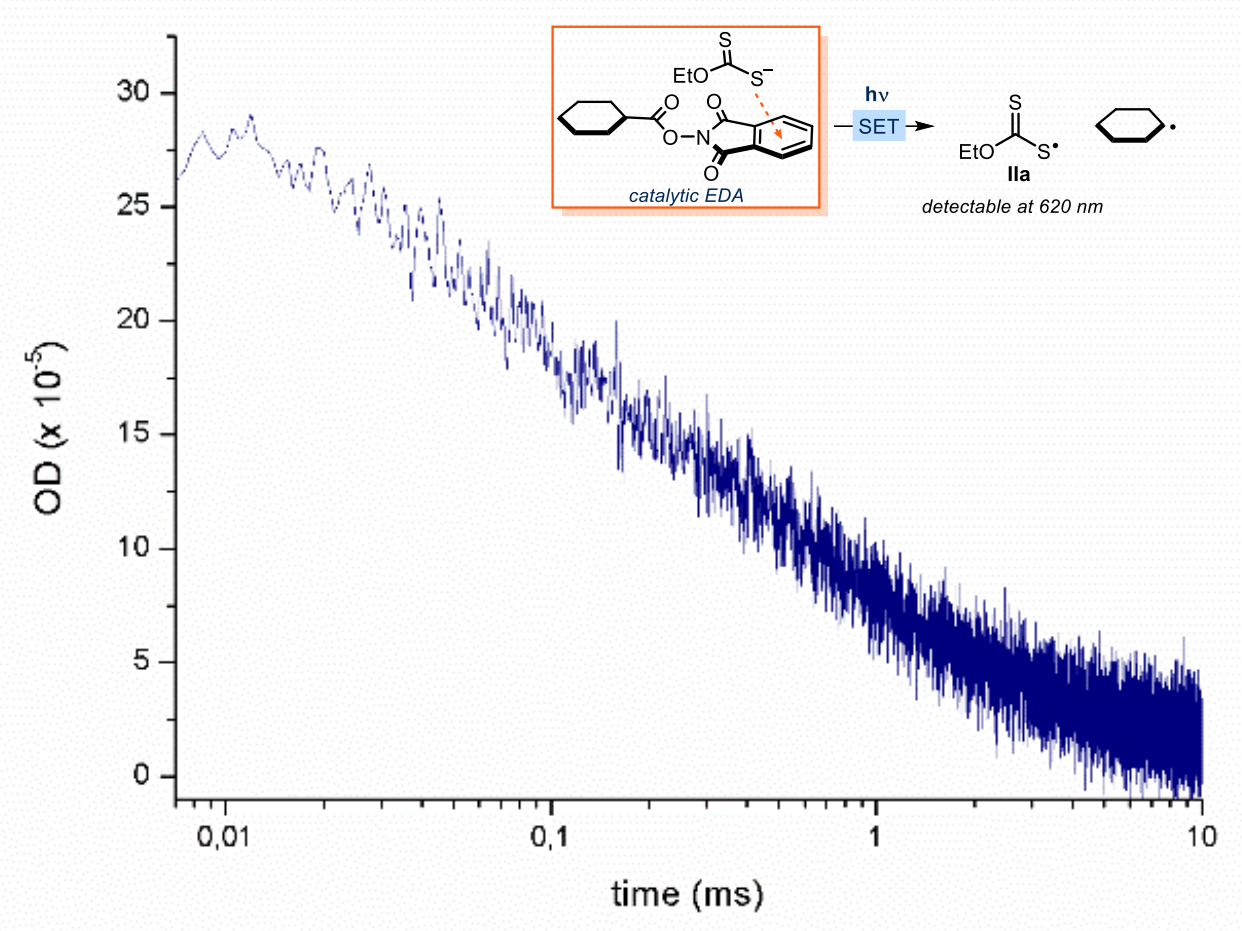

Figure S15: Absorption at $620 \mathrm{~nm}$ of the transient xanthyl radical IIa (blue line) generated upon $355 \mathrm{~nm}$ laser excitation of of a 1:1 mixture of 1a and catalyst B $30 \mathrm{mM}$ in DMA. Note logarithmic scale for time. $\triangle \mathrm{OD}$ : optical density variation.

\section{D4. Cyclic Voltammetry Measurements}

For all cyclic voltammetry (CV) measurements, a glassy carbon disk electrode (diameter $3 \mathrm{~mm}$ ) was used as the working electrode. A silver wire coated with $\mathrm{AgCl}$ immersed in a $3.5 \mathrm{M}$ aqueous solution of $\mathrm{KCl}$ and separated from the analyte by a fritted glass disk was employed as the reference electrode. A Pt wire counter-electrode completed the electrochemical setup. The scan rate used in each $\mathrm{CV}$ experiment is indicated case by case.

Potentials are quoted with the following notation: $E_{\mathrm{p}}{ }^{\mathrm{C}}$ refers to the cathodic peak potential, $E_{\mathrm{p}}{ }^{\mathrm{A}}$ refers to the anodic peak potential, while the $E^{\text {red }}$ value describes the electrochemical properties of the referred compound. 


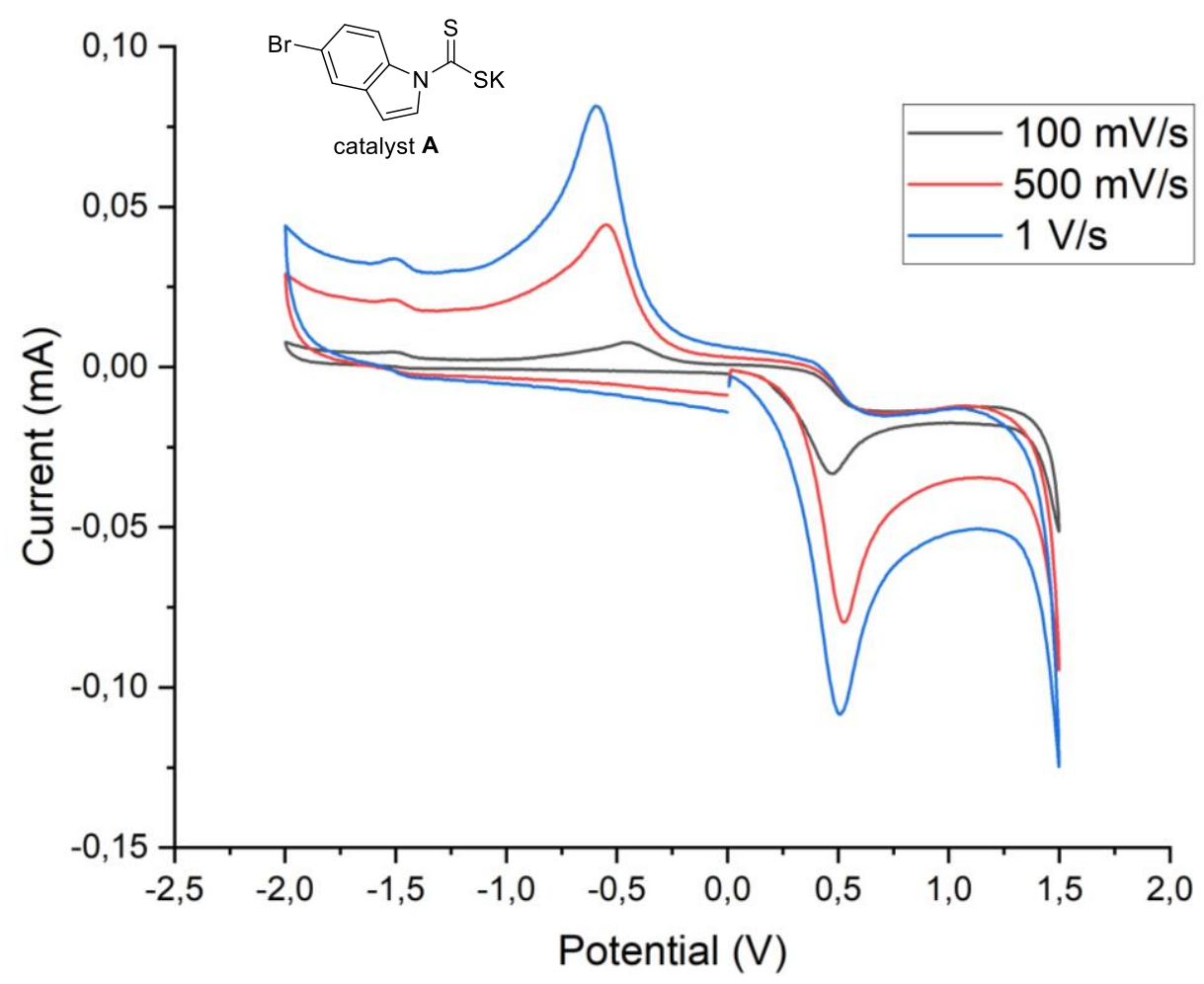

Figure S16: Cyclic voltammogram for catalyst $\mathbf{A}[0.02 \mathrm{M}]$ in $[0.1 \mathrm{M}] \mathrm{TBAPF} 6$ in $\mathrm{CH}_{3} \mathrm{CN}$. Measurement started by oxidation from 0 to $+1.5 \mathrm{~V}$, followed by reduction from $+1.5 \mathrm{~V}$ to $-2.0 \mathrm{~V}$, and finishing at $0 \mathrm{~V}$. Glassy carbon electrode working electrode, $\mathrm{Ag} / \mathrm{AgCl}(\mathrm{KCl} 3.5 \mathrm{M})$ reference electrode, $\mathrm{Pt}$ wire auxiliary electrode. Two irreversible peaks observed increasing with sweep rate.

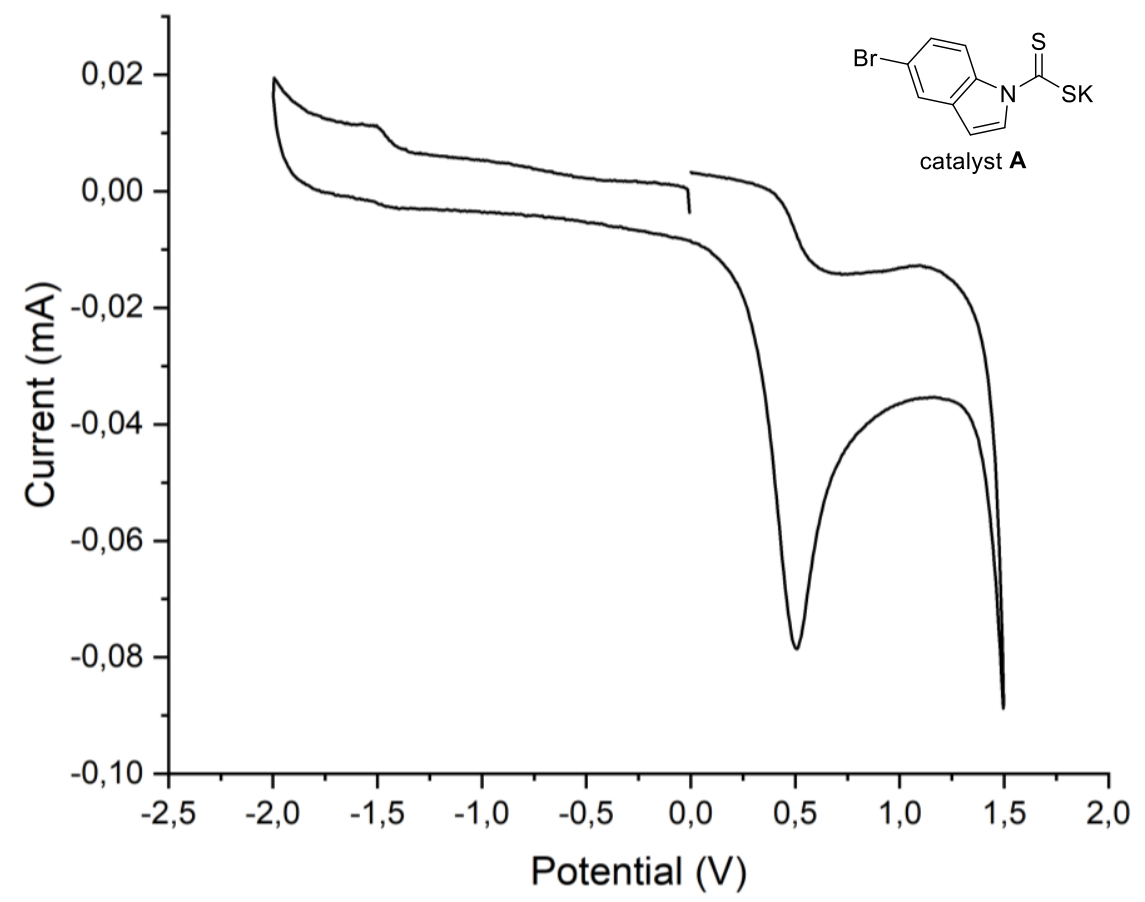

Figure S17: Cyclic voltammogram for catalyst $\mathbf{A}[0.02 \mathrm{M}]$ in $[0.1 \mathrm{M}]$ TBAPF 6 in $\mathrm{CH}_{3} \mathrm{CN}$. Measurement started by reduction from 0 to $-2.0 \mathrm{~V}$, followed by oxidation from $-2.0 \mathrm{~V}$ to $+1.5 \mathrm{~V}$, and finishing at $0 \mathrm{~V}$. Glassy carbon electrode working electrode, $\mathrm{Ag} / \mathrm{AgCl}(\mathrm{KCl} 3.5 \mathrm{M})$ reference electrode, $\mathrm{Pt}$ wire auxiliary electrode. Only one irreversible peak observed. Sweep rate: $500 \mathrm{mV} / \mathrm{s}$. 


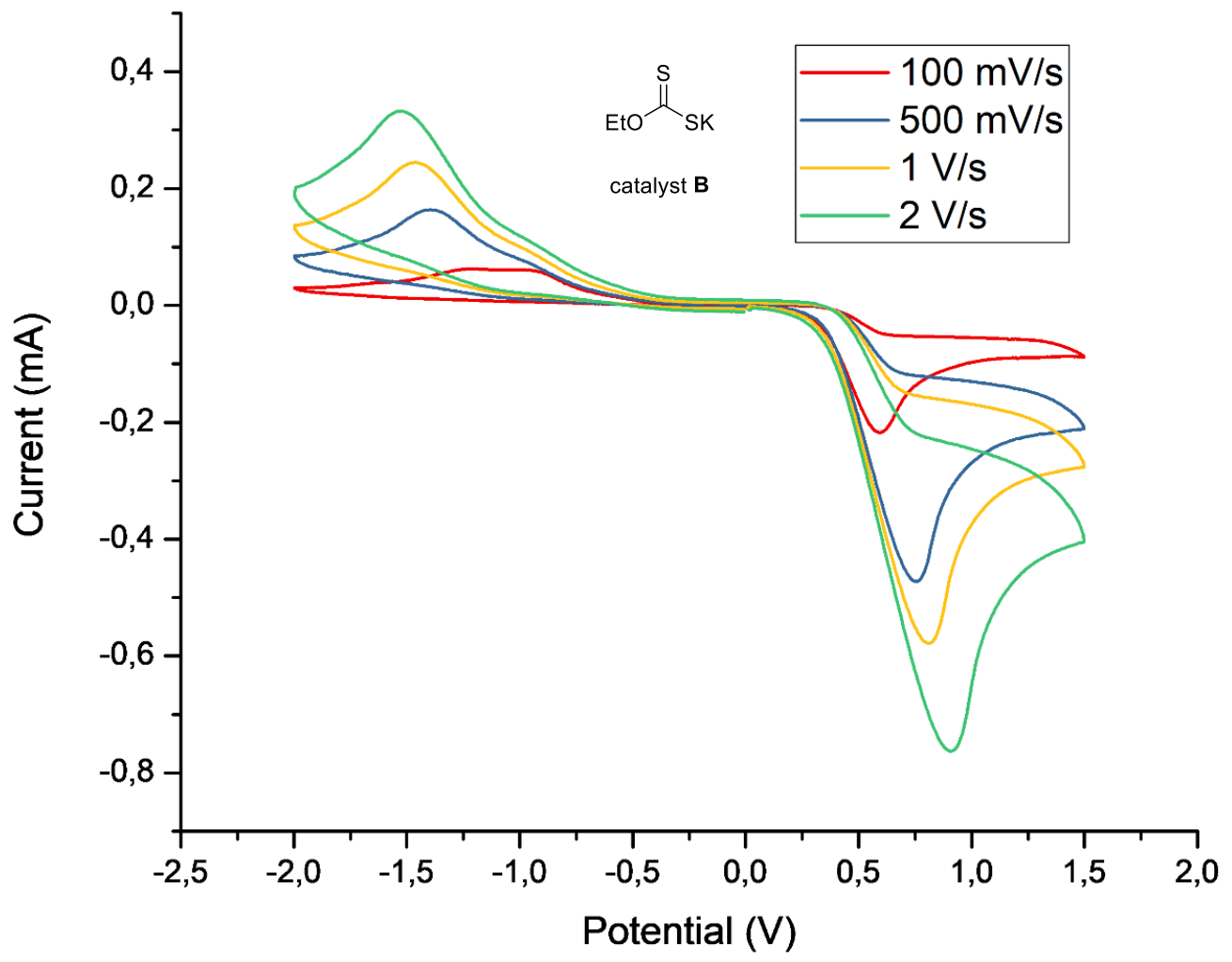

Figure S18: Cyclic voltammogram for catalyst $\mathbf{B}[0.02 \mathrm{M}]$ in $[0.1 \mathrm{M}]$ TBAPF 6 in $\mathrm{CH}_{3} \mathrm{CN}$. Measurement started by oxidation from 0 to $+1.5 \mathrm{~V}$, followed by reduction from $+1.5 \mathrm{~V}$ to $-2.0 \mathrm{~V}$, and finishing at $0 \mathrm{~V}$. Glassy carbon electrode working electrode, $\mathrm{Ag} / \mathrm{AgCl}(\mathrm{KCl} 3.5 \mathrm{M})$ reference electrode, $\mathrm{Pt}$ wire auxiliary electrode. Two irreversible peaks observed increasing with sweep rate.

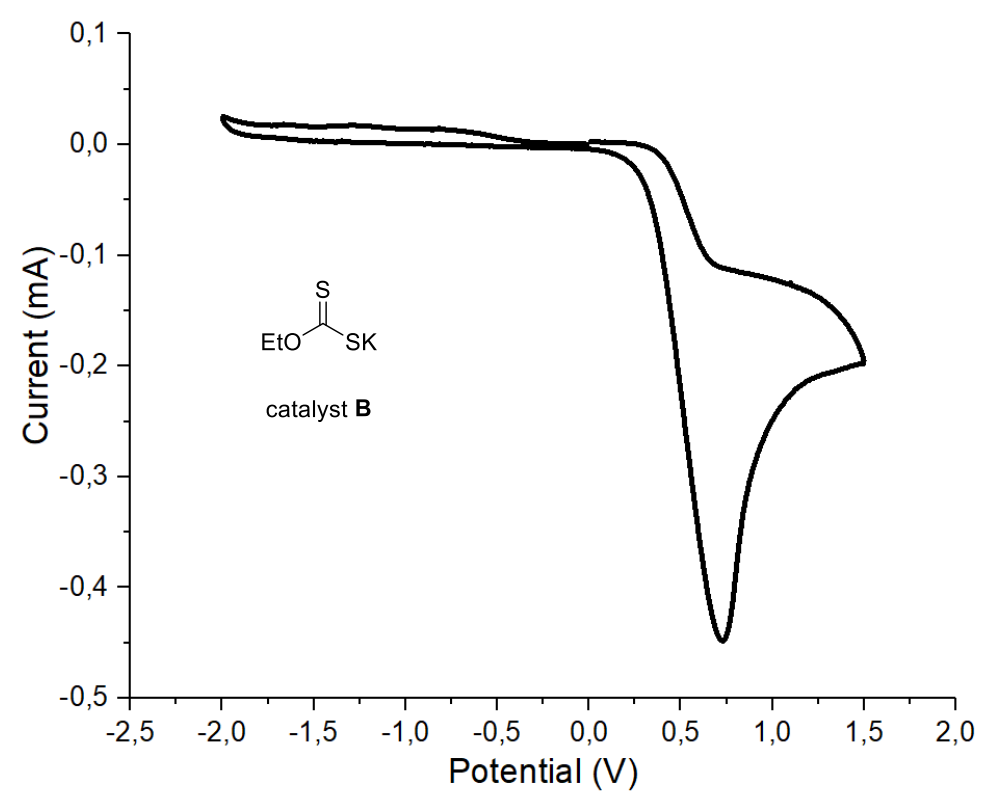

Figure S19: Cyclic voltammogram for catalyst $\mathbf{B}[0.02 \mathrm{M}]$ in $[0.1 \mathrm{M}] \mathrm{TBAPF} 6$ in $\mathrm{CH}_{3} \mathrm{CN}$. Measurement started by reduction from 0 to $-2.0 \mathrm{~V}$, followed by oxidation from $-2.0 \mathrm{~V}$ to $+1.5 \mathrm{~V}$, and finishing at $0 \mathrm{~V}$. Glassy carbon electrode working electrode, $\mathrm{Ag} / \mathrm{AgCl}(\mathrm{KCl} 3.5 \mathrm{M})$ reference electrode, $\mathrm{Pt}$ wire auxiliary electrode. Only one irreversible peak observed. Sweep rate: $500 \mathrm{mV} / \mathrm{s}$. 


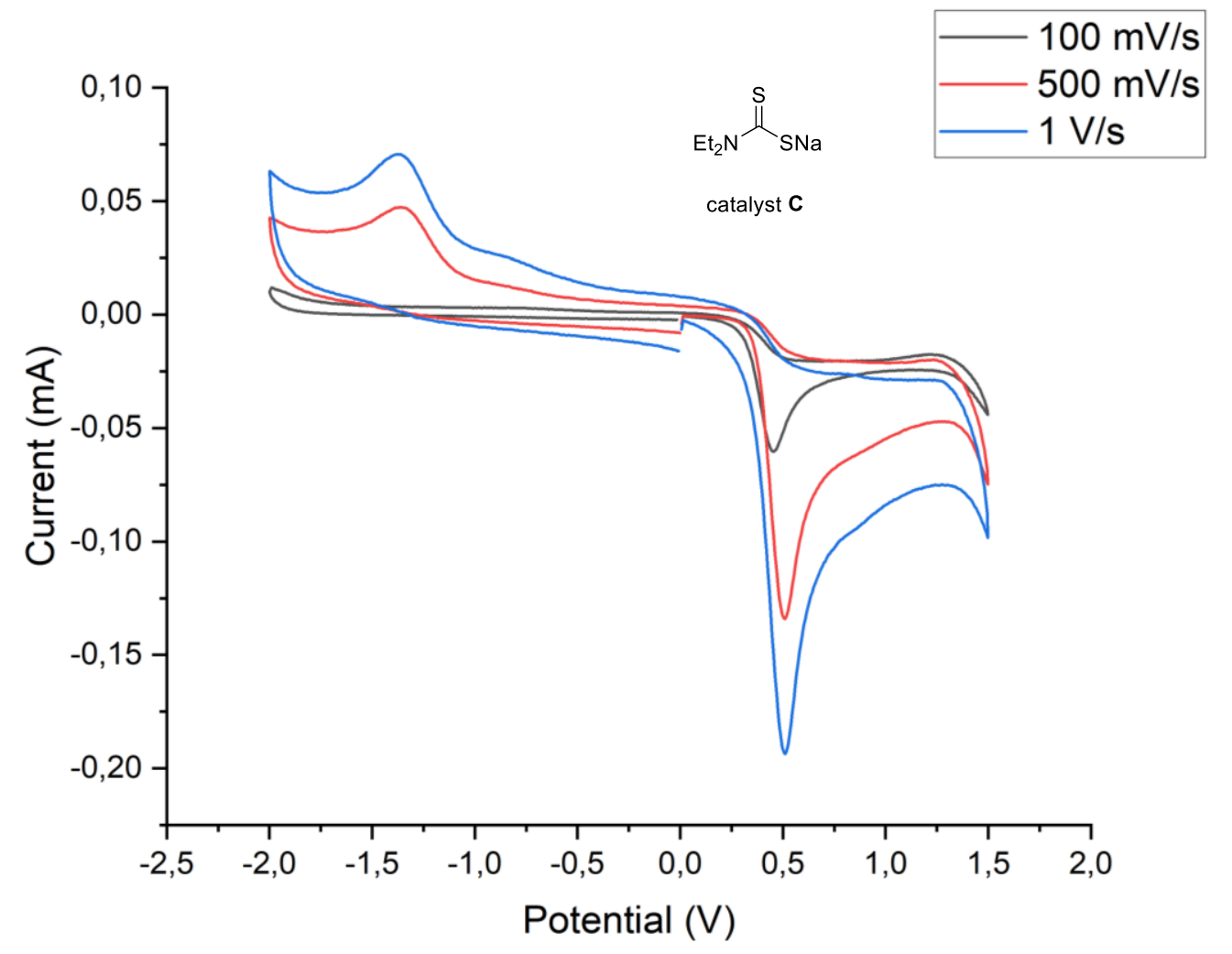

Figure S20: Cyclic voltammogram for catalyst $\mathbf{C}[0.02 \mathrm{M}]$ in $[0.1 \mathrm{M}] \mathrm{TBAPF} 6$ in $\mathrm{CH}_{3} \mathrm{CN}$. Measurement started by oxidation from 0 to $+1.5 \mathrm{~V}$, followed by reduction from $+1.5 \mathrm{~V}$ to $-2.0 \mathrm{~V}$, and finishing at $0 \mathrm{~V}$. Glassy carbon electrode working electrode, $\mathrm{Ag} / \mathrm{AgCl}(\mathrm{KCl} 3.5 \mathrm{M})$ reference electrode, $\mathrm{Pt}$ wire auxiliary electrode. Two irreversible peaks observed increasing with sweep rate.

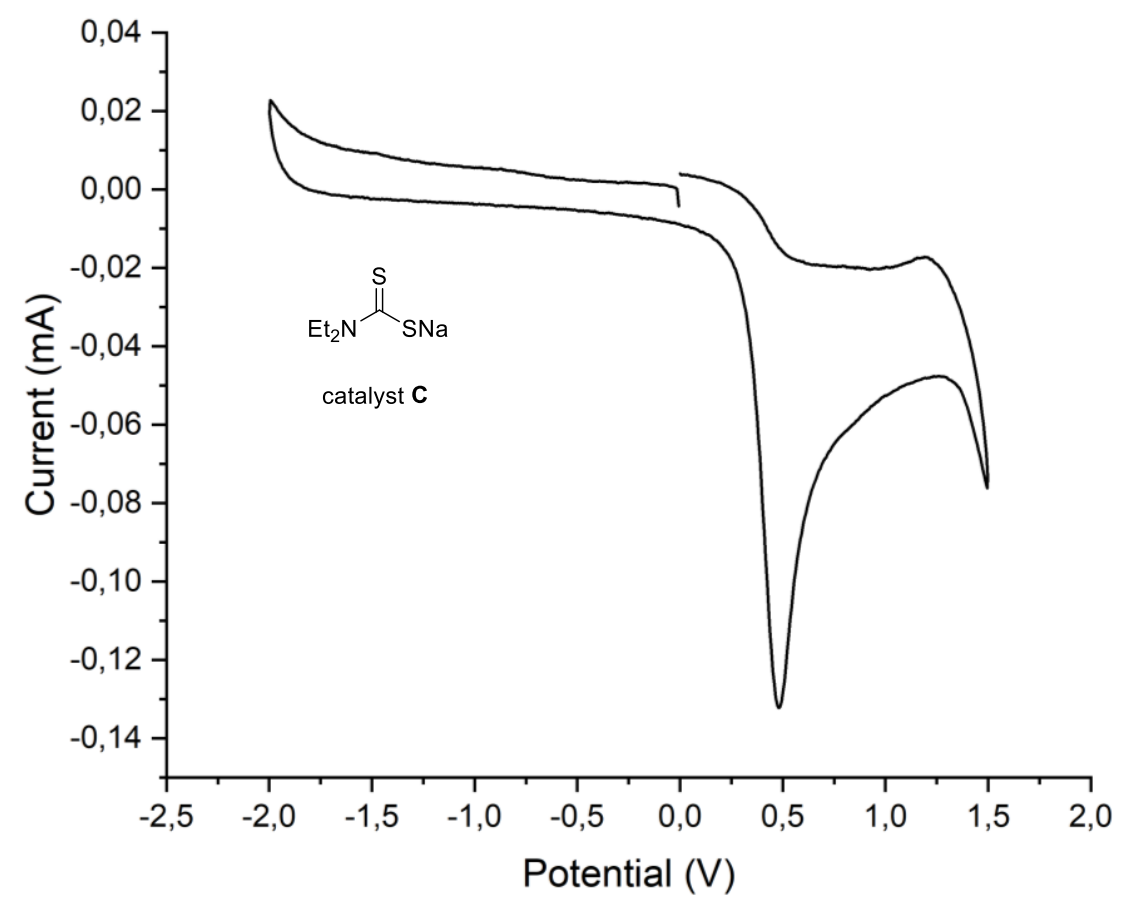

Figure S21: Cyclic voltammogram for catalyst $\mathbf{C}[0.02 \mathrm{M}]$ in $[0.1 \mathrm{M}] \mathrm{TBAPF} 6$ in $\mathrm{CH}_{3} \mathrm{CN}$. Measurement started by reduction from 0 to $-2.0 \mathrm{~V}$, followed by oxidation from $-2.0 \mathrm{~V}$ to $+1.5 \mathrm{~V}$, and finishing at $0 \mathrm{~V}$. Glassy carbon electrode working electrode, $\mathrm{Ag} / \mathrm{AgCl}(\mathrm{KCl} 3.5 \mathrm{M})$ reference electrode, $\mathrm{Pt}$ wire auxiliary electrode. Only one irreversible peak observed. Sweep rate: $500 \mathrm{mV} / \mathrm{s}$. 


\section{D5. Quantum Yield Determination}

\section{D5.1 Giese addition}

A ferrioxalate actinometer solution was prepared by following the Hammond variation of the Hatchard and Parker procedure outlined in the Handbook of Photochemistry. ${ }^{34}$ The ferrioxalate actinometer solution measures the decomposition of ferric ions to ferrous ions, which are complexed by 1,10-phenanthroline and monitored by UV/Vis absorbance at $510 \mathrm{~nm}$. The moles of iron-phenanthroline complex formed are related to moles of photons absorbed. The following solutions were prepared and stored in a dark laboratory (red light):

1. Potassium ferrioxalate solution: $294.8 \mathrm{mg}$ of potassium ferrioxalate (commercially available from Alfa Aesar) and $139 \mu \mathrm{L}$ of sulfuric acid (96\%) were added to a $50 \mathrm{~mL}$ volumetric flask, and filled to the mark with water (HPLC grade).

2. Phenanthroline solution: $0.2 \%$ by weight of 1,10 -phenanthroline in water $(100 \mathrm{mg}$ in $50 \mathrm{~mL}$ volumetric flask).

3. Buffer solution: $2.47 \mathrm{~g}$ of NaOAc and $0.5 \mathrm{~mL}$ of sulfuric acid (96\%) were added to a $50 \mathrm{~mL}$ volumetric flask and filled to the mark with water (HPLC grade).

The actinometry measurements were done as follows:

1. $1 \mathrm{~mL}$ of the actinometer solution was added to a Schlenk tube (diameter $=12 \mathrm{~mm})$. The Schlenk tube was placed in one of the positions of the 3D printed reactor (Figure S2). The solution was irradiated at $460 \mathrm{~nm}$. This procedure was repeated 4 times, quenching the solutions after different time intervals: $1 \mathrm{sec}, 2 \mathrm{sec}, 4 \mathrm{sec}$, and $8 \mathrm{sec}$.

2. Then $1 \mathrm{~mL}$ of the model reaction following general procedure $\mathrm{A}$ with $\mathbf{1 a}(0.10 \mathrm{mmol})$ and $\mathbf{2 a}$ as substrates was placed in a Schlenk tube, degassed via argon bubbling, placed in the irradiation set up and irradiated for 15 minutes. This procedure was performed a total of four times with different irradiation times ( $30 \mathrm{~min}, 45 \mathrm{~min}, 60 \mathrm{~min}$ ).

3. After irradiation, the actinometer solutions were removed and placed in a $10 \mathrm{~mL}$ volumetric flask containing $0.5 \mathrm{~mL}$ of 1,10-phenanthroline solution and $2 \mathrm{~mL}$ of buffer solution. These flasks were filled to the mark with water (HPLC grade).

4. The UV-Vis spectra of the complexed actinometer samples were recorded for each time interval. The absorbance of the complexed actinometer solution was monitored at $510 \mathrm{~nm}$.

The moles of $\mathrm{Fe}^{2+}$ formed for each sample is determined using Beers' Law (Eq. 1):

$$
\text { Mols of } \mathrm{Fe}(\mathrm{II})=V_{1} \times V_{3} \times \Delta A(510 \mathrm{~nm}) / 10^{3} \times V_{2} \times l \times \varepsilon(510 \mathrm{~nm})(\text { Eq. 1) }
$$

where $V_{1}$ is the irradiated volume $(1 \mathrm{~mL}), V_{2}$ is the aliquot of the irradiated solution taken for the determination of the ferrous ions $(1 \mathrm{~mL}), \mathrm{V}_{3}$ is the final volume after complexation with phenanthroline $(10 \mathrm{~mL}), 1$ is the optical path-length of the irradiation cell $(1 \mathrm{~cm}), \Delta \mathrm{A}(510 \mathrm{~nm})$ is the optical difference in absorbance between the irradiated solution and the one stored in the dark, $\varepsilon(510 \mathrm{~nm})$ is the extinction coefficient the complex Fe(phen $)_{3}{ }^{2+}$ at $510 \mathrm{~nm}(11100 \mathrm{~L} \mathrm{~mol}-1 \mathrm{~cm} 1)$. The moles of $\mathrm{Fe} 2+$ formed (x) are plotted as a function of time (t). The slope of this line was correlated to the moles of incident photons by unit of time (q0 n,p) by the use of the following Equation 2:

$$
\Phi(\lambda)=d x / d t \text { qn,p } 0[1-10-A(\lambda)](\text { Eq. 2) }
$$

where $\mathrm{dx} / \mathrm{dt}$ is the rate of change of a measurable quantity (spectral or any other property), the quantum yield $(\Phi)$ for $\mathrm{Fe}^{2+}$ at $458 \mathrm{~nm}$ is $1.1,{ }^{35}\left[1-10^{-\mathrm{A}(\lambda)}\right]$ is the ratio of absorbed photons by the solution, and $\mathrm{A}(\lambda)$ is the absorbance of the actinometer at the wavelength used to carry out the experiments $(460 \mathrm{~nm})$. The absorbance at $460 \mathrm{~nm} \mathrm{A(460)}$ was measured using a Shimadzu 
2401PC UV-Vis spectrophotometer in a $10 \mathrm{~mm}$ path quartz cuvette, obtaining an absorbance of $0.183 . \mathrm{q}_{\mathrm{n}, \mathrm{p}}^{0}$, which is the photon flux, was determined to be $5.3 \times 10^{-7}$.

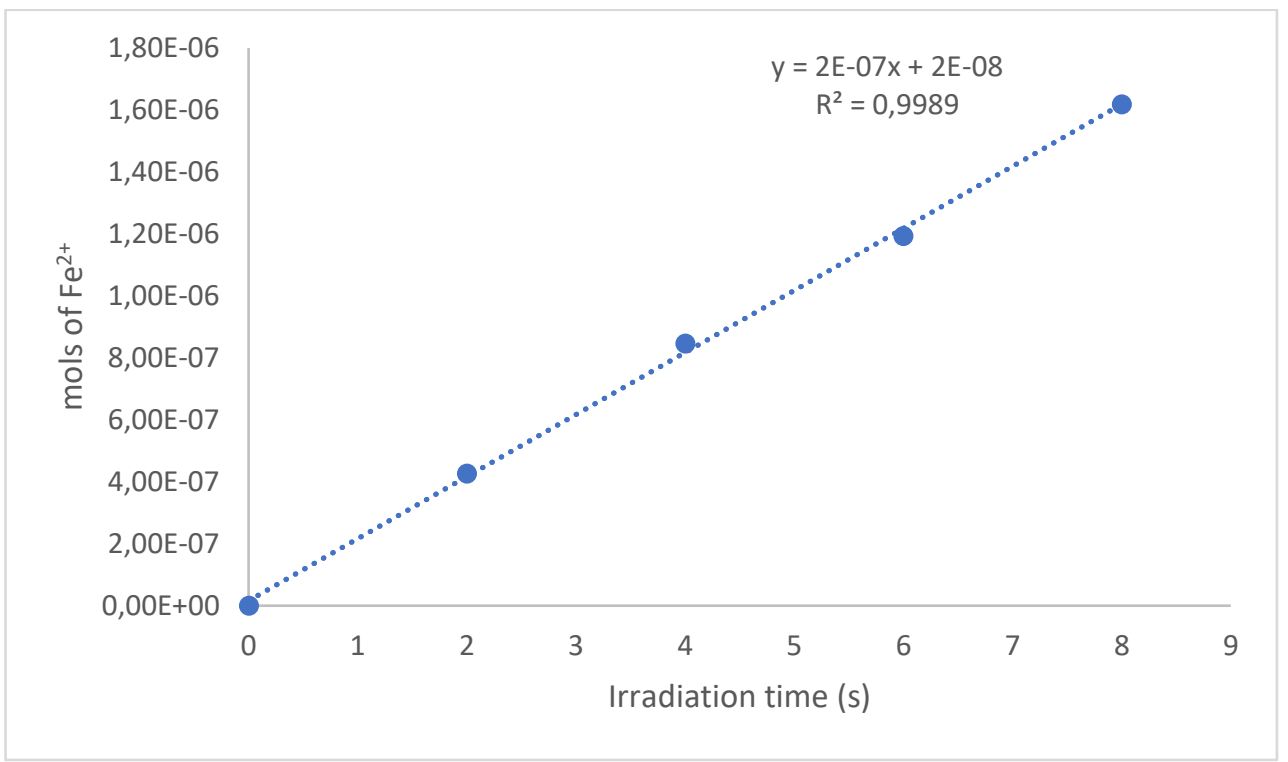

Figure S22: Plot of mols of $\mathrm{Fe}^{2+}$ formed vs irradiation time. Slope of the line correlates to the moles of incident photons by unit of time.

The moles of product 3a formed for the model reaction were determined by GC measurement (FID detector) using 1,3,5-trimethoxybenzene as internal standard. The moles of product per unit of time are related to the number of photons absorbed.

The photons absorbed are correlated to the number of incident photons by the use of Equation 1. According to this, if we plot the moles of product (y) versus the moles of incident photons (q0 $\mathrm{n}, \mathrm{p} \cdot \mathrm{dt})$, the slope is equal to: $\Phi \cdot\left(1-10^{-\mathrm{A}(460 \mathrm{~nm})}\right)$, where $\Phi$ is the quantum yield to be determined and $\mathrm{A}(460 \mathrm{~nm})$ is the absorption of the reaction under study. $\mathrm{A}(460 \mathrm{~nm})$ was measured using a Shimadzu 2401PC UV-Vis spectrophotometer in $10 \mathrm{~mm}$ path quartz. An absorbance of 0.049 was determined for the model reaction mixture (1:4 dilution). The quantum yield $(\Phi)_{\text {cat. }}$ of the photochemical transformation was measured to be 0.01 .

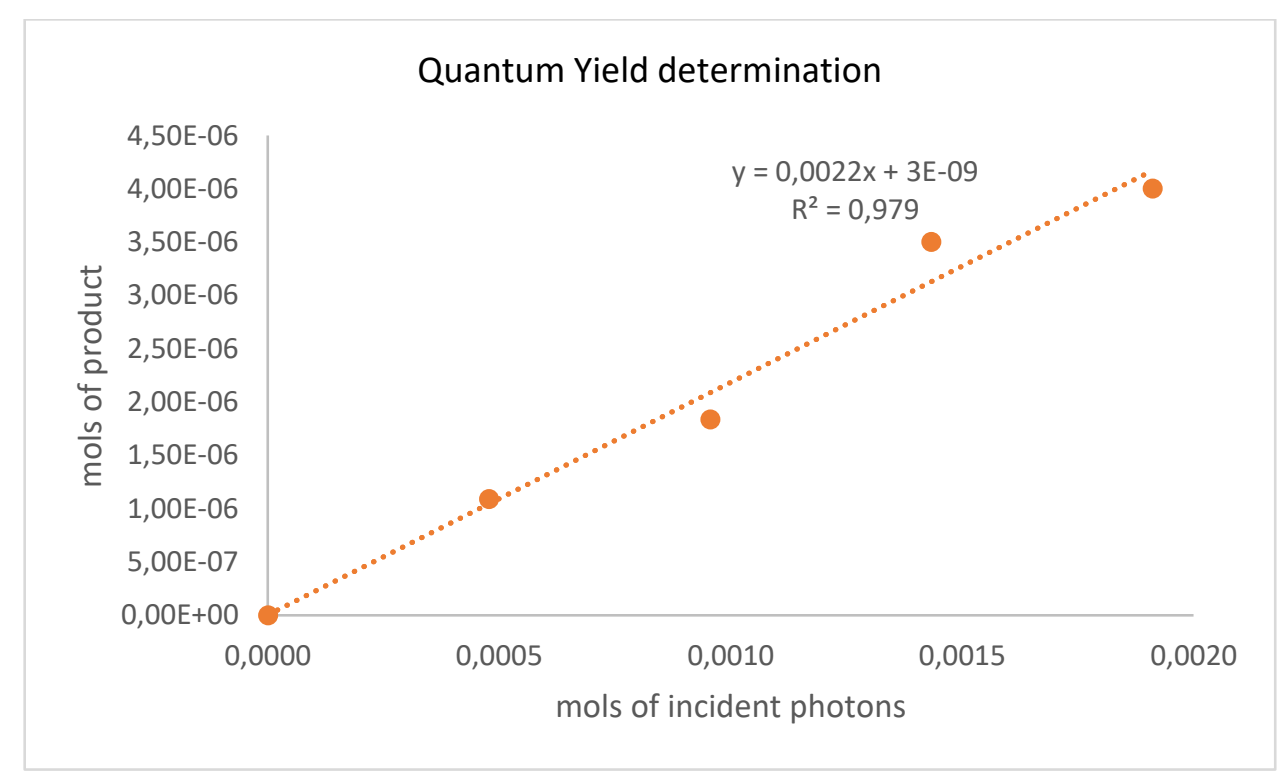

Figure S23: Plot of mols of incident photons vs mols of product formed. Slope of the line correlates to quantum yield of the photochemical transformation. 


\section{D5.2 Barton decarboxylation}

A ferrioxalate actinometer solution was prepared by following the Hammond variation of the Hatchard and Parker procedure outlined in the Handbook of Photochemistry. ${ }^{34}$ The ferrioxalate actinometer solution measures the decomposition of ferric ions to ferrous ions, which are complexed by 1,10-phenanthroline and monitored by UV/Vis absorbance at $510 \mathrm{~nm}$. The moles of iron-phenanthroline complex formed are related to moles of photons absorbed. The following solutions were prepared and stored in a dark laboratory (red light):

1. Potassium ferrioxalate solution: $294.8 \mathrm{mg}$ of potassium ferrioxalate (commercially available from Alfa Aesar) and $139 \mu \mathrm{L}$ of sulfuric acid (96\%) were added to a $50 \mathrm{~mL}$ volumetric flask and filled to the mark with water (HPLC grade).

2. Phenanthroline solution: $0.2 \%$ by weight of 1,10 -phenanthroline in water $(100 \mathrm{mg}$ in $50 \mathrm{~mL}$ volumetric flask).

3. Buffer solution: $2.47 \mathrm{~g}$ of NaOAc and $0.5 \mathrm{~mL}$ of sulfuric acid $(96 \%)$ were added to a $50 \mathrm{~mL}$ volumetric flask and filled to the mark with water (HPLC grade).

The actinometry measurements were done as follows:

1. $1 \mathrm{~mL}$ of the actinometer solution was added to a Schlenk tube (diameter $=12 \mathrm{~mm}$ ). The Schlenk tube was placed in one of the positions of the 3D printed reactor (Figure S2). The solution was irradiated at $460 \mathrm{~nm}$. This procedure was repeated 4 times, quenching the solutions after different time intervals: $1 \mathrm{sec}, 2 \mathrm{sec}, 4 \mathrm{sec}$, and $8 \mathrm{sec}$.

2. Then $1 \mathrm{~mL}$ of the model reaction following general procedure $\mathrm{F}$ starting from isolated $\mathbf{1 a}$ $(0.10 \mathrm{mmol})$ as substrate was placed in a Schlenk tube, degassed via argon bubbling, placed in the irradiation set up and irradiated for 15 minutes. This procedure was performed a total of four times with different irradiation times ( $30 \mathrm{~min}, 60 \mathrm{~min}, 120 \mathrm{~min}$ ).

3. After irradiation, the actinometer solutions were removed and placed in a $10 \mathrm{~mL}$ volumetric flask containing $0.5 \mathrm{~mL}$ of 1,10-phenanthroline solution and $2 \mathrm{~mL}$ of buffer solution. These flasks were filled to the mark with water (HPLC grade).

4. The UV-Vis spectra of the complexed actinometer samples were recorded for each time interval. The absorbance of the complexed actinometer solution was monitored at $510 \mathrm{~nm}$.

The moles of $\mathrm{Fe}^{2+}$ formed for each sample is determined using Beers' Law (Eq. 1) :

$$
\text { Mols of } \mathrm{Fe}(\mathrm{II})=V_{1} \times V_{3} \times \Delta A(510 \mathrm{~nm}) / 10^{3} \times V_{2} \times l \times \varepsilon(510 \mathrm{~nm})(\text { Eq. 1) }
$$

where $\mathrm{V}_{1}$ is the irradiated volume $(1 \mathrm{~mL}), \mathrm{V}_{2}$ is the aliquot of the irradiated solution taken for the determination of the ferrous ions $(1 \mathrm{~mL}), \mathrm{V}_{3}$ is the final volume after complexation with phenanthroline $(10 \mathrm{~mL}), 1$ is the optical path-length of the irradiation cell $(1 \mathrm{~cm}), \Delta \mathrm{A}(510 \mathrm{~nm})$ is the optical difference in absorbance between the irradiated solution and the one stored in the dark, $\varepsilon(510 \mathrm{~nm})$ is the extinction coefficient the complex Fe(phen $)_{3}{ }^{2+}$ at $510 \mathrm{~nm}(11100 \mathrm{~L} \mathrm{~mol}-1 \mathrm{~cm} 1)$. The moles of $\mathrm{Fe} 2+$ formed (x) are plotted as a function of time ( $\mathrm{t}$ ). The slope of this line was correlated to the moles of incident photons by unit of time (q0 n,p) by the use of the following Equation 2:

$$
\Phi(\lambda)=d x / d t \text { qn,p } 0[1-10-A(\lambda)](\text { Eq. } 2)
$$

where $\mathrm{dx} / \mathrm{dt}$ is the rate of change of a measurable quantity (spectral or any other property), the quantum yield $(\Phi)$ for $\mathrm{Fe}^{2+}$ at $458 \mathrm{~nm}$ is $1.1,{ }^{35}\left[1-10^{-\mathrm{A}(\lambda)}\right]$ is the ratio of absorbed photons by the solution, and $\mathrm{A}(\lambda)$ is the absorbance of the actinometer at the wavelength used to carry out the experiments $(460 \mathrm{~nm})$. The absorbance at $460 \mathrm{~nm} \mathrm{~A}(460)$ was measured using a Shimadzu 2401PC UV-Vis spectrophotometer in a $10 \mathrm{~mm}$ path quartz cuvette, obtaining an absorbance of 0.183. $\mathrm{q}_{\mathrm{n}, \mathrm{p}}^{0}$, which is the photon flux, was determined to be $5.2 \times 10^{-7}$. 


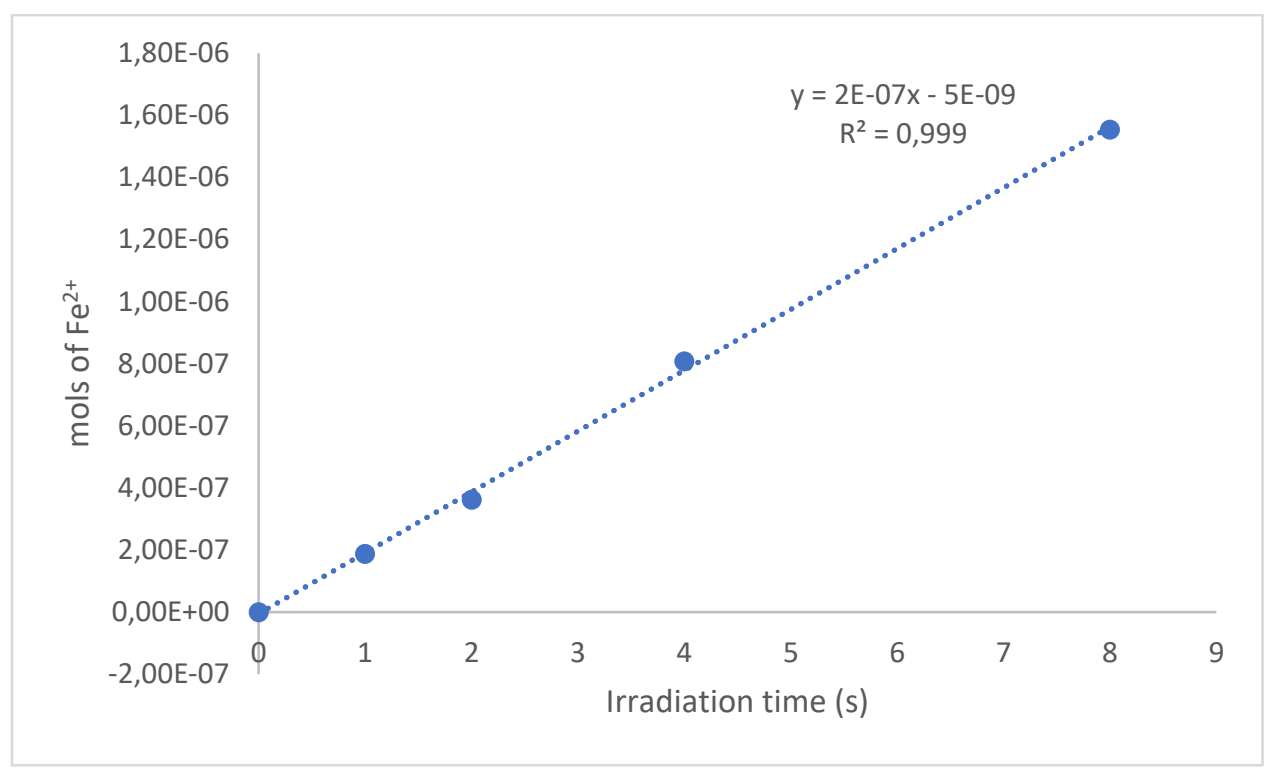

Figure S24: Plot of mols of $\mathrm{Fe}^{2+}$ formed vs irradiation time. Slope of the line correlates to the moles of incident photons by unit of time.

The moles of product $\mathbf{6 b}$ formed for the model reaction were determined by GC measurement (FID detector) using 1,3,5-trimethoxybenzene as internal standard. The moles of product per unit of time are related to the number of photons absorbed.

The photons absorbed are correlated to the number of incident photons by the use of Equation 1. According to this, if we plot the moles of product (y) versus the moles of incident photons (q0 $\mathrm{n}, \mathrm{p} \cdot \mathrm{dt})$, the slope is equal to: $\Phi \cdot\left(1-10^{-\mathrm{A}(460 \mathrm{~nm})}\right)$, where $\Phi$ is the quantum yield to be determined and $\mathrm{A}(460 \mathrm{~nm})$ is the absorption of the reaction under study. $\mathrm{A}(460 \mathrm{~nm})$ was measured using a Shimadzu 2401PC UV-Vis spectrophotometer in $10 \mathrm{~mm}$ path quartz. An absorbance of 0.052 was determined for the model reaction mixture. The quantum yield $(\Phi)_{\text {cat. }}$ of the photochemical transformation was measured to be 0.01 .

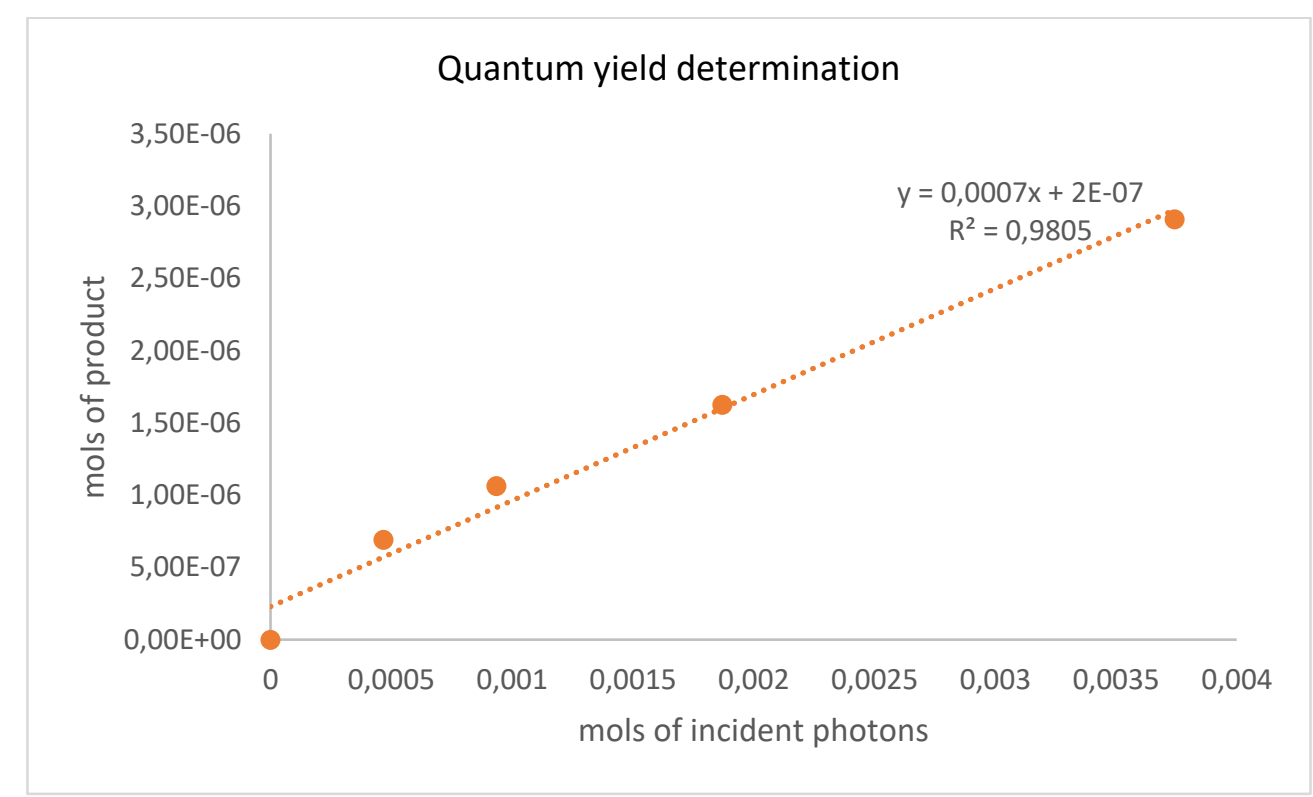

Figure S25: Plot of mols of incident photons vs mols of product formed. Slope of the line correlates to quantum yield of the photochemical transformation. 


\section{D5.3 Alkylation enol ethers}

A ferrioxalate actinometer solution was prepared by following the Hammond variation of the Hatchard and Parker procedure outlined in the Handbook of Photochemistry. ${ }^{34}$ The ferrioxalate actinometer solution measures the decomposition of ferric ions to ferrous ions, which are complexed by 1,10-phenanthroline and monitored by UV/Vis absorbance at $510 \mathrm{~nm}$. The moles of iron-phenanthroline complex formed are related to moles of photons absorbed. The following solutions were prepared and stored in a dark laboratory (red light):

1. Potassium ferrioxalate solution: $294.8 \mathrm{mg}$ of potassium ferrioxalate (commercially available from Alfa Aesar) and $139 \mu \mathrm{L}$ of sulfuric acid (96\%) were added to a $50 \mathrm{~mL}$ volumetric flask and filled to the mark with water (HPLC grade).

2. Phenanthroline solution: $0.2 \%$ by weight of 1,10 -phenanthroline in water $(100 \mathrm{mg}$ in $50 \mathrm{~mL}$ volumetric flask).

3. Buffer solution: $2.47 \mathrm{~g}$ of $\mathrm{NaOAc}$ and $0.5 \mathrm{~mL}$ of sulfuric acid $(96 \%)$ were added to a $50 \mathrm{~mL}$ volumetric flask, and filled to the mark with water (HPLC grade).

The actinometry measurements were done as follows:

1. $1 \mathrm{~mL}$ of the actinometer solution was added to a Schlenk tube (diameter $=12 \mathrm{~mm}$ ). The Schlenk tube was placed in a single HP LED $1.5 \mathrm{~cm}$ away from the light source (irradiance $\left.10 \mathrm{~mW} / \mathrm{cm}^{2}\right) .{ }^{12}$ The solution was irradiated at $460 \mathrm{~nm}$. This procedure was repeated 4 times, quenching the solutions after different time intervals: $5 \mathrm{sec}, 10 \mathrm{sec}, 20 \mathrm{sec}$, and $40 \mathrm{sec}$.

2. Then $1 \mathrm{~mL}$ of the model reaction following general procedure $\mathrm{H}$ with $7 \mathbf{a}(0.10 \mathrm{mmol})$ and $4 \mathbf{l}$ as substrates was placed in a Schlenk tube, degassed via argon bubbling, placed in the irradiation set up and irradiated for 15 minutes. This procedure was performed a total of four times with different irradiation times (30 min, $50 \mathrm{~min}, 70 \mathrm{~min}$ ).

3. After irradiation, the actinometer solutions were removed and placed in a $10 \mathrm{~mL}$ volumetric flask containing $0.5 \mathrm{~mL}$ of 1,10-phenanthroline solution and $2 \mathrm{~mL}$ of buffer solution. These flasks were filled to the mark with water (HPLC grade).

4. The UV-Vis spectra of the complexed actinometer samples were recorded for each time interval. The absorbance of the complexed actinometer solution was monitored at $510 \mathrm{~nm}$.

The moles of $\mathrm{Fe}^{2+}$ formed for each sample is determined using Beers' Law (Eq. 1):

$$
\text { Mols of } \mathrm{Fe}(\mathrm{II})=V_{1} \times V_{3} \times \Delta A(510 \mathrm{~nm}) / 10^{3} \times V_{2} \times l \times \varepsilon(510 \mathrm{~nm})(\text { Eq. } 1)
$$

where $V_{1}$ is the irradiated volume $(1 \mathrm{~mL}), V_{2}$ is the aliquot of the irradiated solution taken for the determination of the ferrous ions $(1 \mathrm{~mL}), \mathrm{V}_{3}$ is the final volume after complexation with phenanthroline $(10 \mathrm{~mL}), 1$ is the optical path-length of the irradiation cell $(1 \mathrm{~cm}), \Delta \mathrm{A}(510 \mathrm{~nm})$ is the optical difference in absorbance between the irradiated solution and the one stored in the dark, $\varepsilon(510 \mathrm{~nm})$ is the extinction coefficient the complex Fe(phen) ${ }_{3}{ }^{2+}$ at $510 \mathrm{~nm}(11100 \mathrm{~L} \mathrm{~mol}-1 \mathrm{~cm} 1)$. The moles of $\mathrm{Fe}^{2}+$ formed (x) are plotted as a function of time $(\mathrm{t})$. The slope of this line was correlated to the moles of incident photons by unit of time (q0 n,p) by the use of the following Equation 2:

$$
\Phi(\lambda)=d x d t \text { qn,p } 0[1-10-A(\lambda)](\text { Eq. } 2)
$$

where $\mathrm{dx} / \mathrm{dt}$ is the rate of change of a measurable quantity (spectral or any other property), the quantum yield $(\Phi)$ for $\mathrm{Fe}^{2+}$ at $458 \mathrm{~nm}$ is $1.1,^{35}\left[1-10^{-\mathrm{A}(\lambda)}\right]$ is the ratio of absorbed photons by the solution, and $\mathrm{A}(\lambda)$ is the absorbance of the actinometer at the wavelength used to carry out the experiments $(460 \mathrm{~nm})$. The absorbance at $460 \mathrm{~nm} \mathrm{A(460)}$ was measured using a Shimadzu 2401PC UV-Vis spectrophotometer in a $10 \mathrm{~mm}$ path quartz cuvette, obtaining an absorbance of 0.148. $\mathrm{q}_{\mathrm{n}, \mathrm{p}}^{0}$, which is the photon flux, was determined to be $1.18 \times 10^{-7}$. 


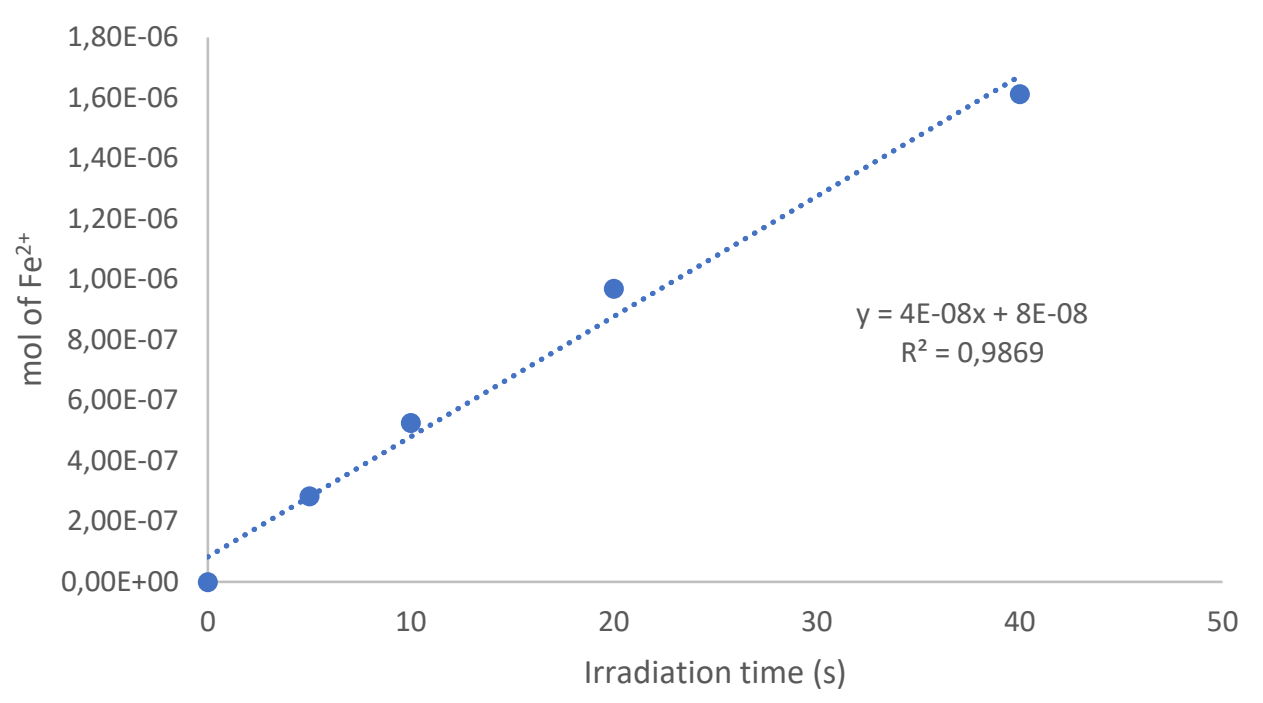

Figure S26: Plot of mols of $\mathrm{Fe}^{2+}$ formed vs irradiation time. Slope of the line correlates to the moles of incident photons by unit of time.

The moles of product $\mathbf{8 h}$ formed for the model reaction were determined by GC measurement (FID detector) using 1,3,5-trimethoxybenzene as internal standard. The moles of product per unit of time are related to the number of photons absorbed.

The photons absorbed are correlated to the number of incident photons by the use of Equation 1 . According to this, if we plot the moles of product (y) versus the moles of incident photons (q0 $\mathrm{n}, \mathrm{p} \cdot \mathrm{dt})$, the slope is equal to: $\Phi \cdot\left(1-10^{-\mathrm{A}(460 \mathrm{~nm})}\right)$, where $\Phi$ is the quantum yield to be determined and $\mathrm{A}(460 \mathrm{~nm})$ is the absorption of the reaction under study. $\mathrm{A}(460 \mathrm{~nm})$ was measured using a Shimadzu 2401PC UV-Vis spectrophotometer in $10 \mathrm{~mm}$ path quartz. An absorbance of 0.018 was determined for the model reaction mixture (1:100 dilution). The quantum yield $(\Phi)_{\text {cat. }}$ of the photochemical transformation was measured to be 0.02 .

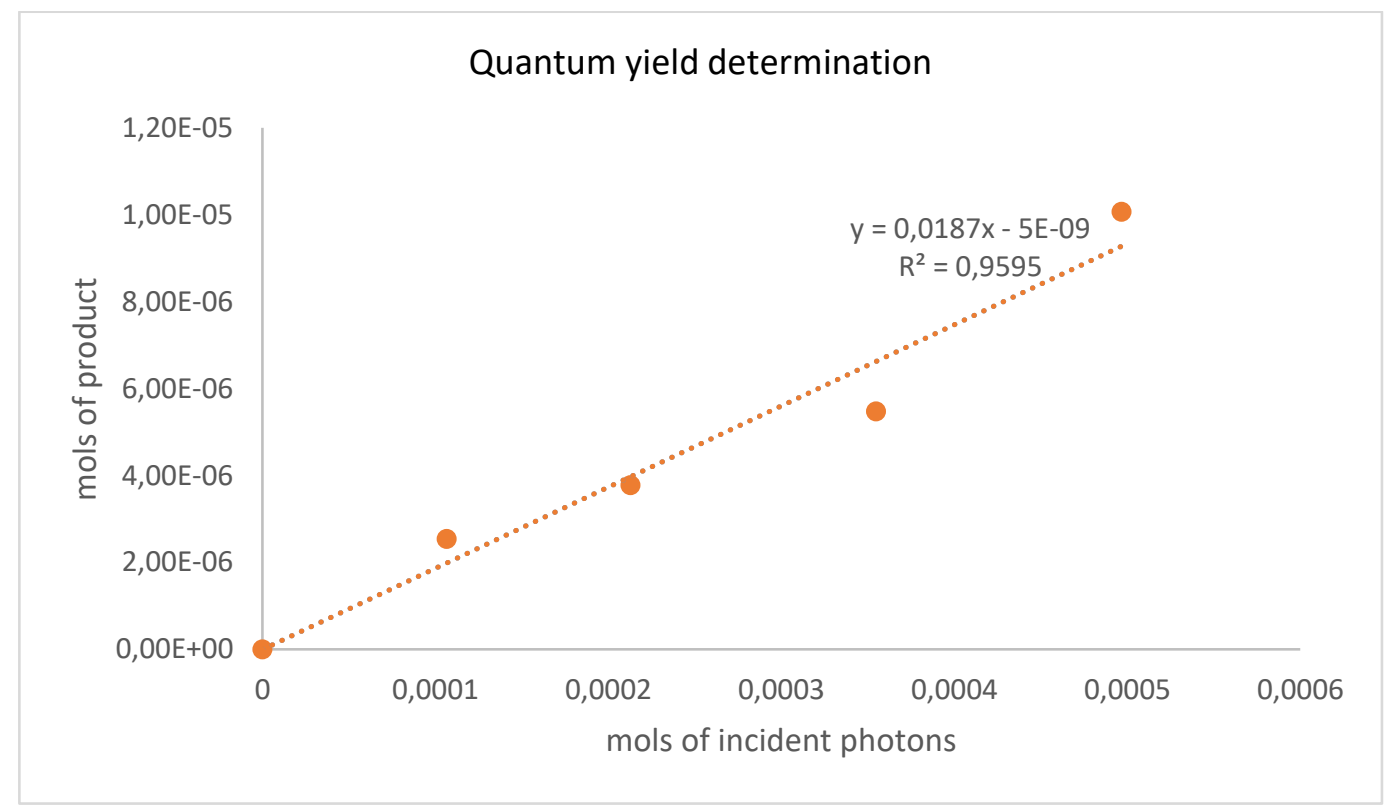

Figure S27: Plot of mols of incident photons vs mols of product formed. Slope of the line correlates to quantum yield of the photochemical transformation. 


\section{D5.4 Minisci reaction}

A ferrioxalate actinometer solution was prepared by following the Hammond variation of the Hatchard and Parker procedure outlined in the Handbook of Photochemistry. ${ }^{34}$ The ferrioxalate actinometer solution measures the decomposition of ferric ions to ferrous ions, which are complexed by 1,10-phenanthroline and monitored by UV/Vis absorbance at $510 \mathrm{~nm}$. The moles of iron-phenanthroline complex formed are related to moles of photons absorbed. The following solutions were prepared and stored in a dark laboratory (red light):

1. Potassium ferrioxalate solution: $294.8 \mathrm{mg}$ of potassium ferrioxalate (commercially available from Alfa Aesar) and $139 \mu \mathrm{L}$ of sulfuric acid $(96 \%)$ were added to a $50 \mathrm{~mL}$ volumetric flask, and filled to the mark with water (HPLC grade).

2. Phenanthroline solution: $0.2 \%$ by weight of 1,10 -phenanthroline in water $(100 \mathrm{mg}$ in $50 \mathrm{~mL}$ volumetric flask).

3. Buffer solution: $2.47 \mathrm{~g}$ of $\mathrm{NaOAc}$ and $0.5 \mathrm{~mL}$ of sulfuric acid $(96 \%)$ were added to a $50 \mathrm{~mL}$ volumetric flask, and filled to the mark with water (HPLC grade).

The actinometry measurements were done as follows:

1. $1 \mathrm{~mL}$ of the actinometer solution was added to a Schlenk tube (diameter $=12 \mathrm{~mm}$ ). The Schlenk tube was placed in one of the position of the 3D printed reactor (Figure S2). The solution was irradiated at $460 \mathrm{~nm}$. This procedure was repeated 4 times, quenching the solutions after different time intervals: $1 \mathrm{sec}, 2 \mathrm{sec}, 4 \mathrm{sec}$, and $8 \mathrm{sec}$.

2. Then $1 \mathrm{~mL}$ of the model reaction following general procedure $\mathrm{J}$ with $\mathbf{1 a}(0.10 \mathrm{mmol})$ and 2 methylquinoline as substrates was placed in a Schlenk tube, degassed via argon bubbling, placed in the irradiation set up and irradiated for 60 minutes. This procedure was performed a total of four times with different irradiation times (90 $\mathrm{min}, 120 \mathrm{~min}, 150 \mathrm{~min}$ ).

3. After irradiation, the actinometer solutions were removed and placed in a $10 \mathrm{~mL}$ volumetric flask containing $0.5 \mathrm{~mL}$ of 1,10-phenanthroline solution and $2 \mathrm{~mL}$ of buffer solution. These flasks were filled to the mark with water (HPLC grade).

4. The UV-Vis spectra of the complexed actinometer samples were recorded for each time interval. The absorbance of the complexed actinometer solution was monitored at $510 \mathrm{~nm}$.

The moles of $\mathrm{Fe}^{2+}$ formed for each sample is determined using Beers' Law (Eq. 1) :

$$
\text { Mols of } \mathrm{Fe}(\mathrm{II})=V_{1} \times V_{3} \times \Delta A(510 \mathrm{~nm}) / 10^{3} \times V_{2} \times l \times \varepsilon(510 \mathrm{~nm})(\text { Eq. 1) }
$$

where $\mathrm{V}_{1}$ is the irradiated volume $(1 \mathrm{~mL}), \mathrm{V}_{2}$ is the aliquot of the irradiated solution taken for the determination of the ferrous ions $(1 \mathrm{~mL}), \mathrm{V}_{3}$ is the final volume after complexation with phenanthroline $(10 \mathrm{~mL}), 1$ is the optical path-length of the irradiation cell $(1 \mathrm{~cm}), \Delta \mathrm{A}(510 \mathrm{~nm})$ is the optical difference in absorbance between the irradiated solution and the one stored in the dark, $\varepsilon(510 \mathrm{~nm})$ is the extinction coefficient the complex Fe(phen $)_{3}{ }^{2+}$ at $510 \mathrm{~nm}(11100 \mathrm{~L} \mathrm{~mol}-1 \mathrm{~cm} 1)$. The moles of $\mathrm{Fe} 2+$ formed (x) are plotted as a function of time ( $\mathrm{t}$ ). The slope of this line was correlated to the moles of incident photons by unit of time (q0 n,p) by the use of the following Equation 2:

$$
\Phi(\lambda)=d x / d t \text { qn,p } 0[1-10-A(\lambda)](\text { Eq. 2) }
$$

where $\mathrm{dx} / \mathrm{dt}$ is the rate of change of a measurable quantity (spectral or any other property), the quantum yield $(\Phi)$ for $\mathrm{Fe}^{2+}$ at $458 \mathrm{~nm}$ is $1.1,{ }^{35}\left[1-10^{-\mathrm{A}(\lambda)}\right]$ is the ratio of absorbed photons by the solution, and $\mathrm{A}(\lambda)$ is the absorbance of the actinometer at the wavelength used to carry out the experiments $(460 \mathrm{~nm})$. The absorbance at $460 \mathrm{~nm} \mathrm{A(460)}$ was measured using a Shimadzu 2401PC UV-Vis spectrophotometer in a $10 \mathrm{~mm}$ path quartz cuvette, obtaining an absorbance of 0.183. $\mathrm{q}_{\mathrm{n}, \mathrm{p}}^{0}$, which is the photon flux, was determined to be $4.68 \times 10^{-7}$. 


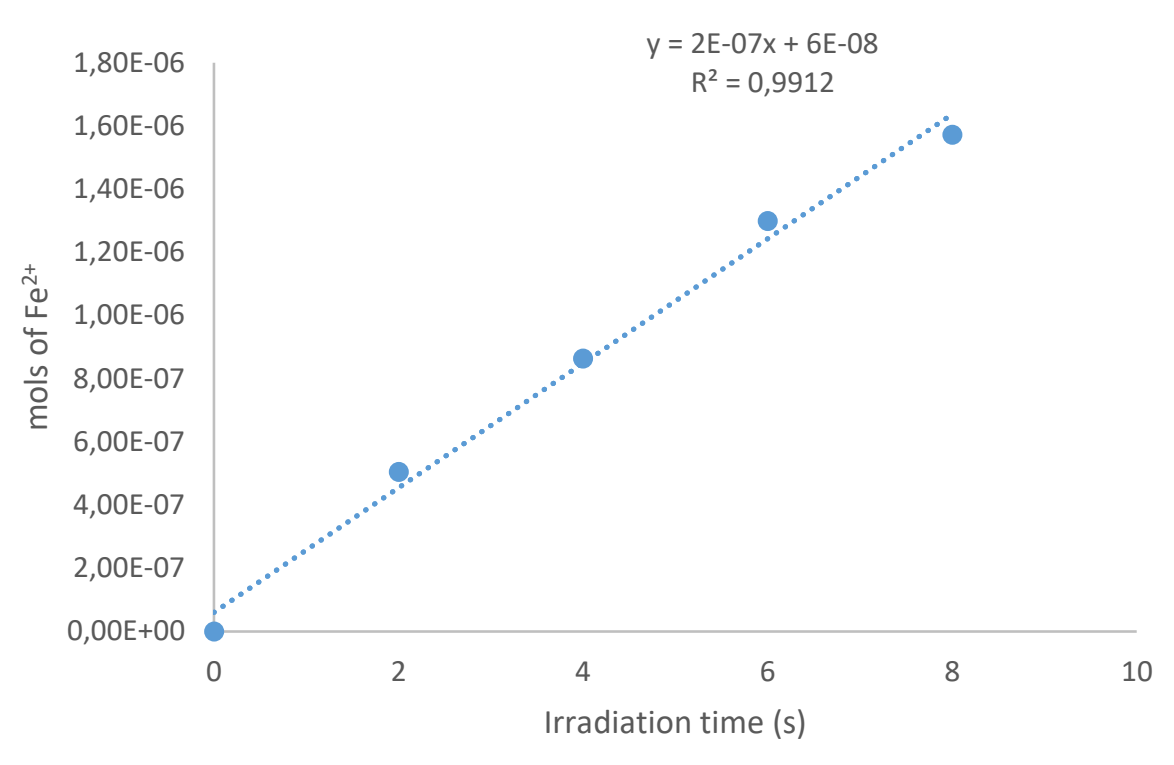

Figure S28: Plot of mols of $\mathrm{Fe}^{2+}$ formed vs irradiation time. Slope of the line correlates to the moles of incident photons by unit of time.

The moles of product $\mathbf{1 1 b}$ formed for the model reaction were determined by GC measurement (FID detector) using 1,3,5-trimethoxybenzene as internal standard. The moles of product per unit of time are related to the number of photons absorbed.

The photons absorbed are correlated to the number of incident photons by the use of Equation 1 . According to this, if we plot the moles of product (y) versus the moles of incident photons (q0 $\mathrm{n}, \mathrm{p} \cdot \mathrm{dt})$, the slope is equal to: $\Phi \cdot\left(1-10^{-\mathrm{A}(460 \mathrm{~nm})}\right)$, where $\Phi$ is the quantum yield to be determined and $\mathrm{A}(460 \mathrm{~nm})$ is the absorption of the reaction under study. $\mathrm{A}(460 \mathrm{~nm})$ was measured using a Shimadzu 2401PC UV-Vis spectrophotometer in $10 \mathrm{~mm}$ path quartz. An absorbance of 0.174 was determined for the model reaction mixture (1:10 dilution). The quantum yield $(\Phi)_{\text {cat. }}$ of the photochemical transformation was measured to be 0.0003 .

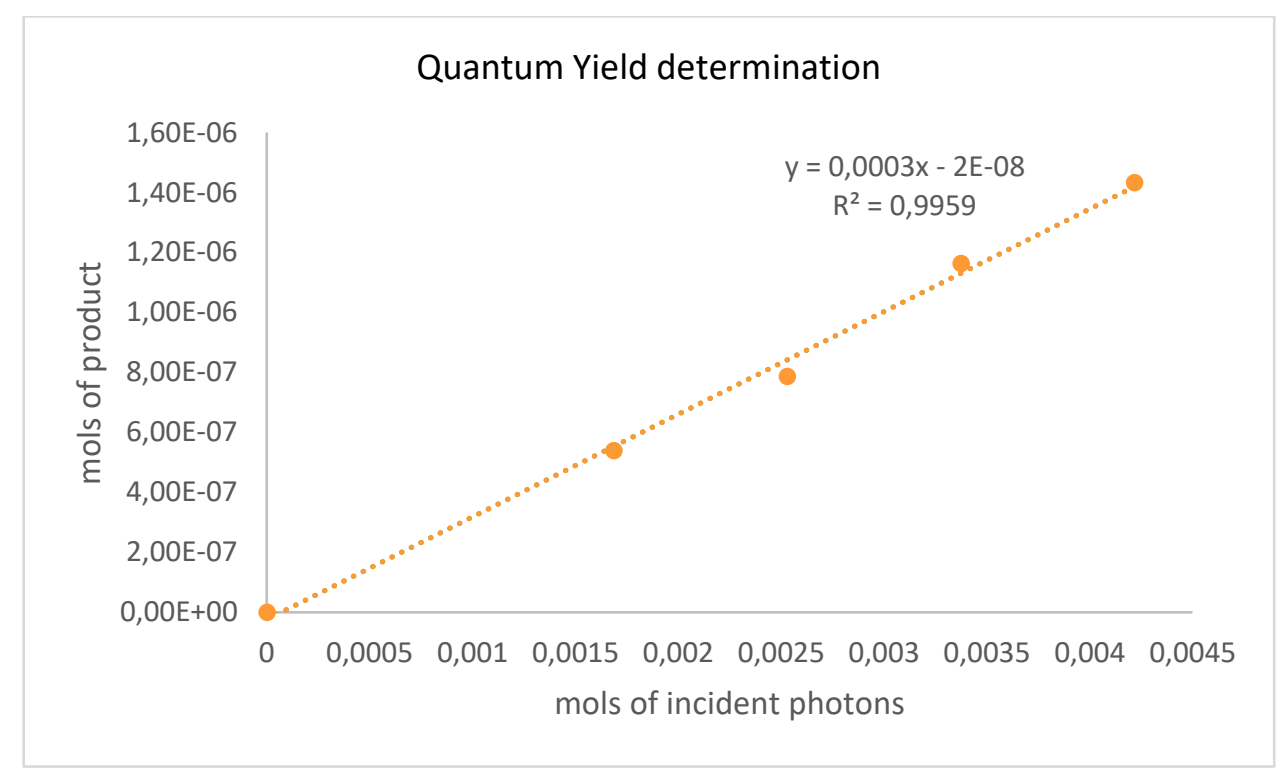

Figure S29: Plot of mols of incident photons vs mols of product formed. Slope of the line correlates to quantum yield of the photochemical transformation. 


\section{E. References}

(1) Qin, T.; Malins, L. R.; Edwards, J. T.; Merchant, R. R.; Novak, A. J. E.; Zhong, J. Z.; Mills, R. B.; Yan, M.; Yuan, C.; Eastgate, M. D.; Baran, P. S., Nickel-Catalyzed Barton Decarboxylation and Giese Reactions: A Practical Take on Classic Transforms. Angew. Chem., Int. Ed. 2017, 56, 260-265.

(2) Huihui, K. M. M.; Caputo, J. A.; Melchor, Z.; Olivares, A. M.; Spiewak, A. M.; Johnson, K. A.; DiBenedetto, T. A.; Kim, S.; Ackerman, L. K. G.; Weix, D. J., Decarboxylative Cross-Electrophile Coupling of N-Hydroxyphthalimide Esters with Aryl Iodides. J. Am. Chem. Soc. 2016, 138, 5016-5019.

(3) Ishii, T.; Kakeno, Y.; Nagao, K.; Ohmiya, H., N-Heterocyclic Carbene-Catalyzed Decarboxylative Alkylation of Aldehydes. J. Am. Chem. Soc. 2019, 141 (9), 3854-3858.

(4) Proctor, R. S. J.; Davis, H. J.; Phipps, R. J., Catalytic enantioselective Minisci-type addition to heteroarenes. Science 2018, 360, 419-422.

(5) Klauck F. J. R.; James M. J.; Glorius F., Deaminative Strategy for the Visible-LightMediated Generation of Alkyl Radicals. Angew. Chem., Int. Ed. 2017, 56, 12336-12339.

(6) Liao J.; Guan W.; Boscoe B. P.; Tucker J. W.; Tomlin J. W.; Garnsey M. R.; Watson M. P., Transforming Benzylic Amines into Diarylmethanes: Cross-Couplings of Benzylic Pyridinium Salts via C-N Bond Activation. Org. Lett. 2018, 20, 3030-3033.

(7) Xia Q.; Li Y.; Wang X.; Dai P.; Deng H.; Zhang W.-H.,Visible Light-Driven $\alpha$-Alkylation of N-Aryl tetrahydroisoquinolines Initiated by Electron Donor-Acceptor Complexes. Org. Lett. 2020, 22, 7290-7294.

(8) Laroche B.; Tang X.; Archer G.; Di Sanza R.; Melchiorre P., Photochemical Chemoselective Alkylation of Tryptophan-Containing Peptides. Org. Lett. 2021, 23, 285 289.

(9) Lai S.-Z.; Yang Y.-M.; Xu H.; Tang Z.-Y; Luo Z., Photoinduced Deaminative Coupling of Alkylpyridium Salts with Terminal Arylalkynes. J. Org. Chem. 2020, 85, 1563815644.

(10) Perrotta D.; Racine S.; Vuilleumier J.; de Nanteuil F.; Waser J., [4 + 2]-Annulations of Aminocyclobutanes. Org. Lett. 2015, 17, 1030-1033.

(11) Li Y.; Liu J., Zhao S., Du X.; Guo M.; Zhao W.; Tang X.; Wang G., Copper-Catalyzed Fluoroolefination of Silyl Enol Ethers and Ketones toward the Synthesis of $\beta$ Fluoroenones. Org. Lett. 2018, 20, 917-920.

(12) Spinnato D.; Schweitzer-Chaput B.; Goti G.; M. Ošeka; Melchiorre P., A Photochemical Organocatalytic Strategy for the $\alpha$-Alkylation of Ketones by using Radicals. Angew. Chem., Int. Ed. 2020, 59, 9485-9490.

(13) Perez C.; Barkley-Levenson A. M.; Dick B. L.; Glatt P. F.; Martinez Y; Siegel D.; Momper J. D.; Palmer A. A.; Cohen S. M., Metal-Binding Pharmacophore Library Yields the Discovery of a Glyoxalase 1 Inhibitor. J. Med. Chem. 2019, 62, 1609-1625.

(14) Davies J.; Svejstrup T. D.; Reina D. F.; Sheikh N. S.; Leonori D., Visible-Light-Mediated Synthesis of Amidyl Radicals: TransitionMetal-Free Hydroamination and N-Arylation Reactions. J. Am. Chem. Soc. 2016, 138, 8092-8095.

(15) Chen, X.; Luo, X.; Peng, X.; Guo, J.; Zai, J.; Wang, P., Catalyst-Free Decarboxylation of Carboxylic Acids and Deoxygenation of Alcohols by Electro-Induced Radical Formation. Chem. Eur. J. 2020, 26, 3226-3230.

(16) Bhunia, A.; Bergander, K.; Studer, A., Cooperative Palladium/Lewis Acid-Catalyzed Transfer Hydrocyanation of Alkenes and Alkynes Using 1-Methylcyclohexa-2,5-diene1-carbonitrile. J. Am. Chem. Soc. 2018, 140, 16353-16359. 
(17) Chu, L.; Ohta, C.; Zuo, Z.; MacMillan, D. W. C., Carboxylic Acids as A Traceless Activation Group for Conjugate Additions: A Three-Step Synthesis of ( \pm )-Pregabalin. $J$. Am. Chem. Soc. 2014, 136, 10886-10889.

(18) Li, D.; Ma, T.-K.; Scott, R. J.; Wilden, J. D. Electrochemical radical reactions of alkyl iodides: a highly efficient, clean, green alternative to tin reagents, Chem. Sci. 2020, 11, 5333-5338.

(19) Yoshimi, Y.; Masuda, M.; Mizunashi, T.; Nishikawa, K.; Maeda, K.; Koshida, N.; Itou, T.; Morita, T.; Hatanaka, M., Inter- and Intramolecular Addition Reactions of ElectronDeficient Alkenes with Alkyl Radicals, Generated by SET-Photochemical Decarboxylation of Carboxylic Acids, Serve as a Mild and Efficient Method for the Preparation of $\gamma$-Amino Acids and Macrocyclic Lactones. Org. Lett. 2009, 11, 4652-4655.

(20) Steiman, T. J.; Liu, J.; Mengiste, A.; Doyle, A. G., Synthesis of $\beta$-Phenethylamines via $\mathrm{Ni} /$ Photoredox Cross-Electrophile Coupling of Aliphatic Aziridines and Aryl Iodides. $J$. Am. Chem. Soc. 2020, 142, 7598-7605.

(21) Vechorkin, O.; Proust, V.; Hu, X., Functional Group Tolerant Kumada-Corriu-Tamao Coupling of Nonactivated Alkyl Halides with Aryl and Heteroaryl Nucleophiles: Catalysis by a Nickel Pincer Complex Permits the Coupling of Functionalized Grignard Reagents. J. Am. Chem. Soc. 2009, 131, 9756-9766.

(22) Percec, V.; Peterca, M.; Sienkowska, M. J.; Ilies, M. A.; Aqad, E.; Smidrkal, J.; Heiney, P. A., Synthesis and Retrostructural Analysis of Libraries of AB3 and Constitutional Isomeric AB2 Phenylpropyl Ether-Based Supramolecular Dendrimers. J. Am. Chem. Soc. 2006, 128, 3324-3334.

(23) Dong S.; Wu G.; Yuan X.; Zou C.; Ye J., Visible-light photoredox catalyzed hydroacylation of electron-deficient alkenes: carboxylic anhydride as an acyl radical source. Org. Chem. Front. 2017, 4, 2230-2234.

(24) Ding B.; Zhang Z.; Liu Y.; Sugiya M.; Imamoto T.; Zhang W., Chemoselective Transfer Hydrogenation of $\alpha, \beta$-Unsaturated Ketones Catalyzed by Pincer-Pd Complexes Using Alcohol as a Hydrogen Source. Org. Lett. 2013, 15, 3690-3693.

(25) Kong, W.; Yu, C.; An, H.; Song, Q., Photoredox-Catalyzed Decarboxylative Alkylation of Silyl Enol Ethers To Synthesize Functionalized Aryl Alkyl Ketones. Org. Lett. 2018, 20 (2), 349-352.

(26) Zheng, Y.-L.; Xie, P.-P.; Daneshfar, O.; Houk, K. N.; Hong, X.; Newman, S. G., Direct Synthesis of Ketones from Methyl Esters by Nickel-Catalyzed Suzuki-Miyaura Coupling. Angew. Chem. Int. Ed. 2021, 60 (24), 13476-13483.

(27) McCallum, T.; Barriault, L., Direct alkylation of heteroarenes with unactivated bromoalkanes using photoredox gold catalysis. Chem. Sci. 2016, 7, 4754-4758.

(28) Zidan, M.; Morris, A. O.; McCallum, T.; Barriault, L., The Alkylation and Reduction of Heteroarenes with Alcohols Using Photoredox Catalyzed Hydrogen Atom Transfer via Chlorine Atom Generation. Eur. J. Org. Chem. 2020, 1453-1458.

(29) Wei, W.; Wang, L.; Bao, P.; Shao, Y.; Yue, H.; Yang, D.; Yang, X.; Zhao, X.; Wang, H., Metal-Free C(sp2)-H/N-H Cross-Dehydrogenative Coupling of Quinoxalinones with Aliphatic Amines under Visible-Light Photoredox Catalysis. Org. Lett. 2018, 20, 71257130.

(30) Li, G.-X.; Morales-Rivera, C. A.; Wang, Y.; Gao, F.; He, G.; Liu, P.; Chen, G., Photoredox-mediated Minisci C-H alkylation of N-heteroarenes using boronic acids and hypervalent iodine. Chem. Sci. 2016, 7, 6407-6412. 
(31) Jacquet J.; Cheaib K.; Ren Y.; Vezin H.; Orio M.; Blanchard S.; Fensterbank L.; Desage El Murr M., Circumventing Intrinsic Metal Reactivity: Radical Generation with RedoxActive Ligands. Chem. Eur. J. 2017, 23, 15030-15034.

(32) Tobisu M.; Koh K.; Furukawa T.; Chatani N., Modular Synthesis of Phenanthridine Derivatives by Oxidative Cyclization of 2-Isocyanobiphenyls with Organoboron Reagents. Angew. Chem., Int. Ed. 2012, 51, 11363-11366.

(33) de Pedro Beato, E.; Mazzarella, D.; Balletti, M.; Melchiorre, P., Photochemical generation of acyl and carbamoyl radicals using a nucleophilic organic catalyst: applications and mechanism thereof. Chem. Sci. 2020, 11, 6312-6324.

(34) Murov, S. L. Ed. Handbook of Photochemistry (Marcel Dekker, New York, 1973).

(35) Holubov C. A.; Langford C. H., Wavelength and temperature dependence in the photolysis of the chemical actinometer, potassium trisoxalatoferrate(III), at longer wavelengths. Inorg. Chim. Acta. 1981, 53, 59-60. 
${ }^{1} \mathrm{H}$ NMR (500 MHz, $\mathrm{CDCl}_{3}$ )

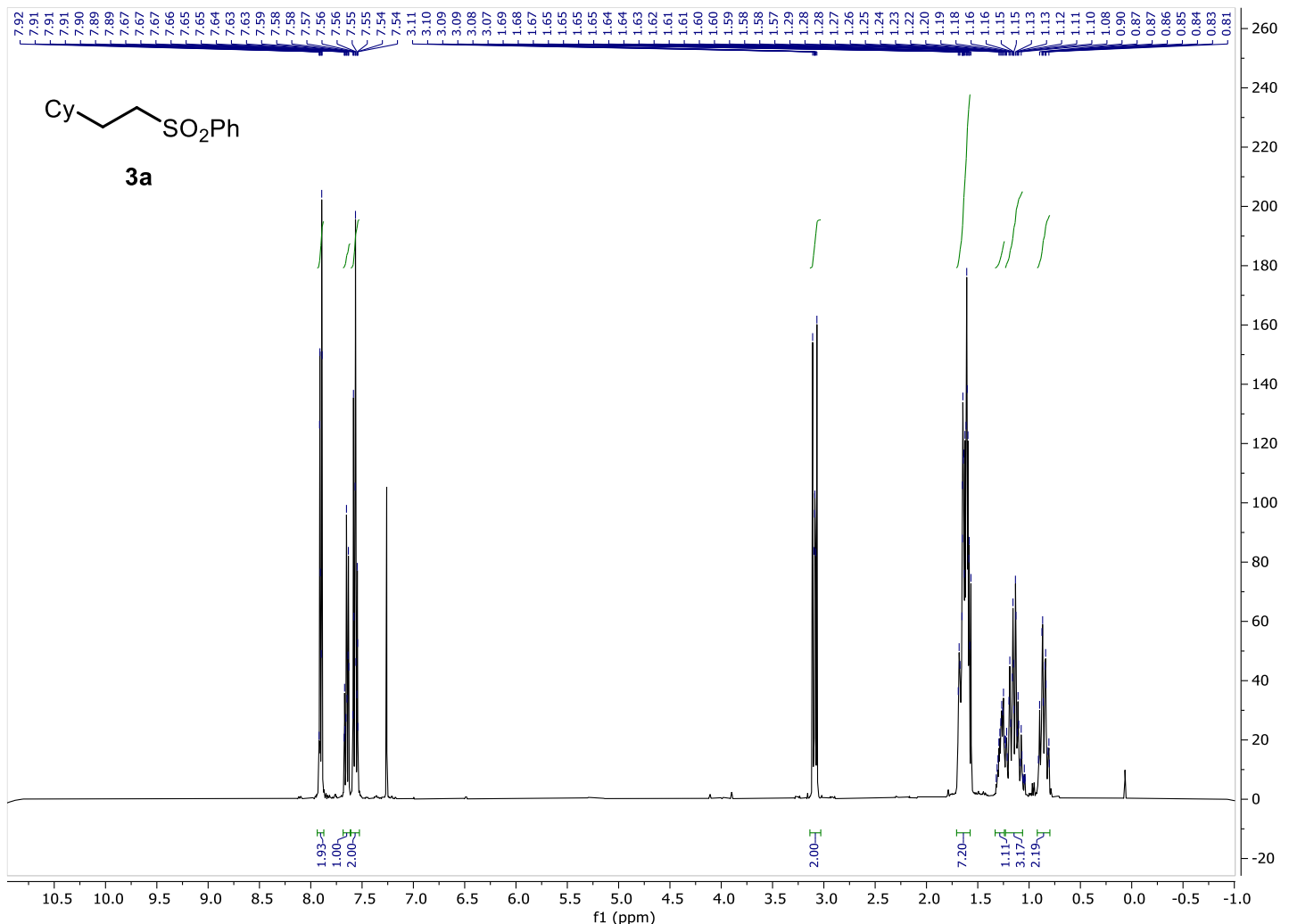

${ }^{13} \mathrm{C}$ NMR (126 MHz, $\left.\mathrm{CDCl}_{3}\right)$

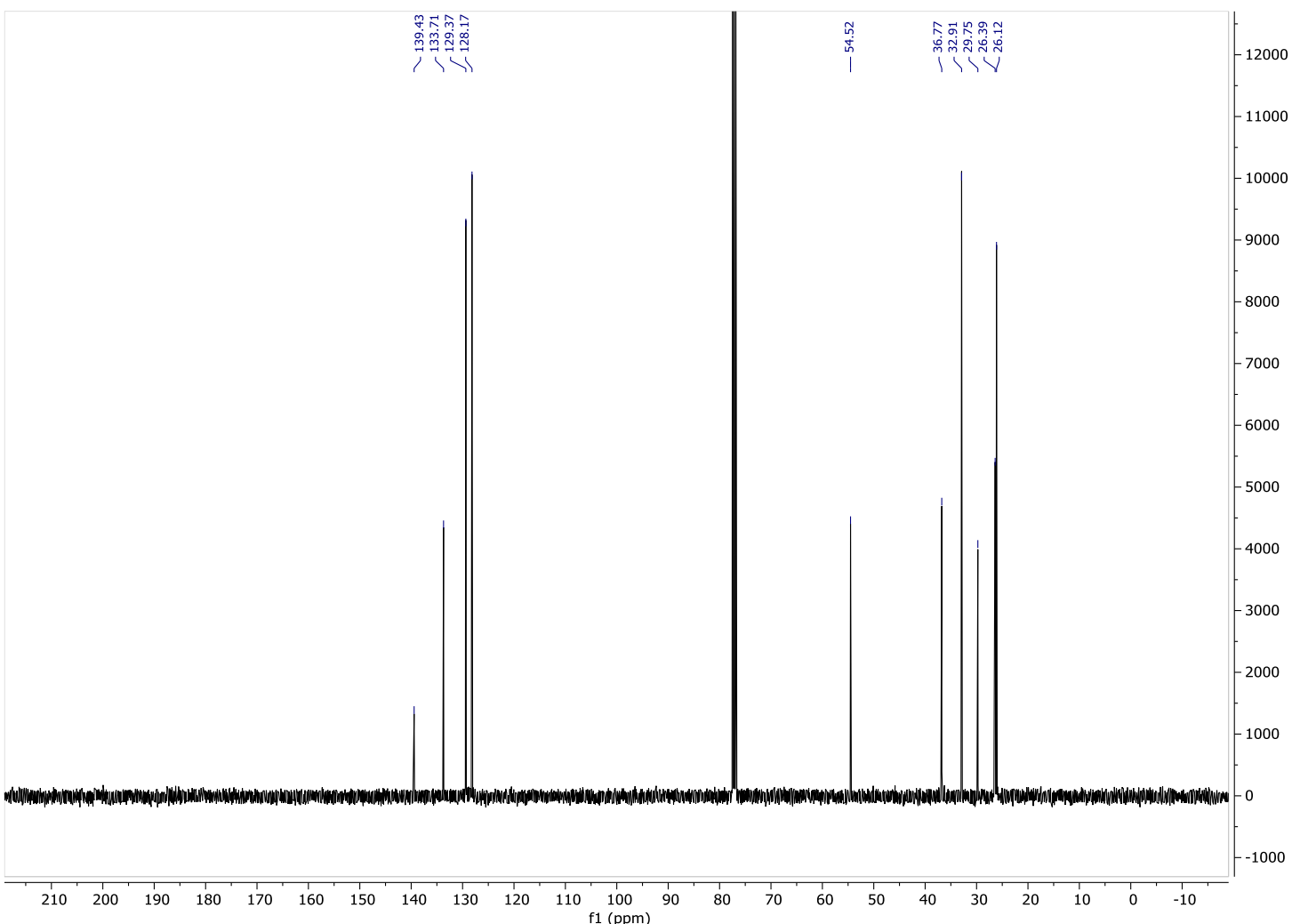


${ }^{1} \mathrm{H}$ NMR (400 MHz, $\left.\mathrm{CDCl}_{3}\right)$

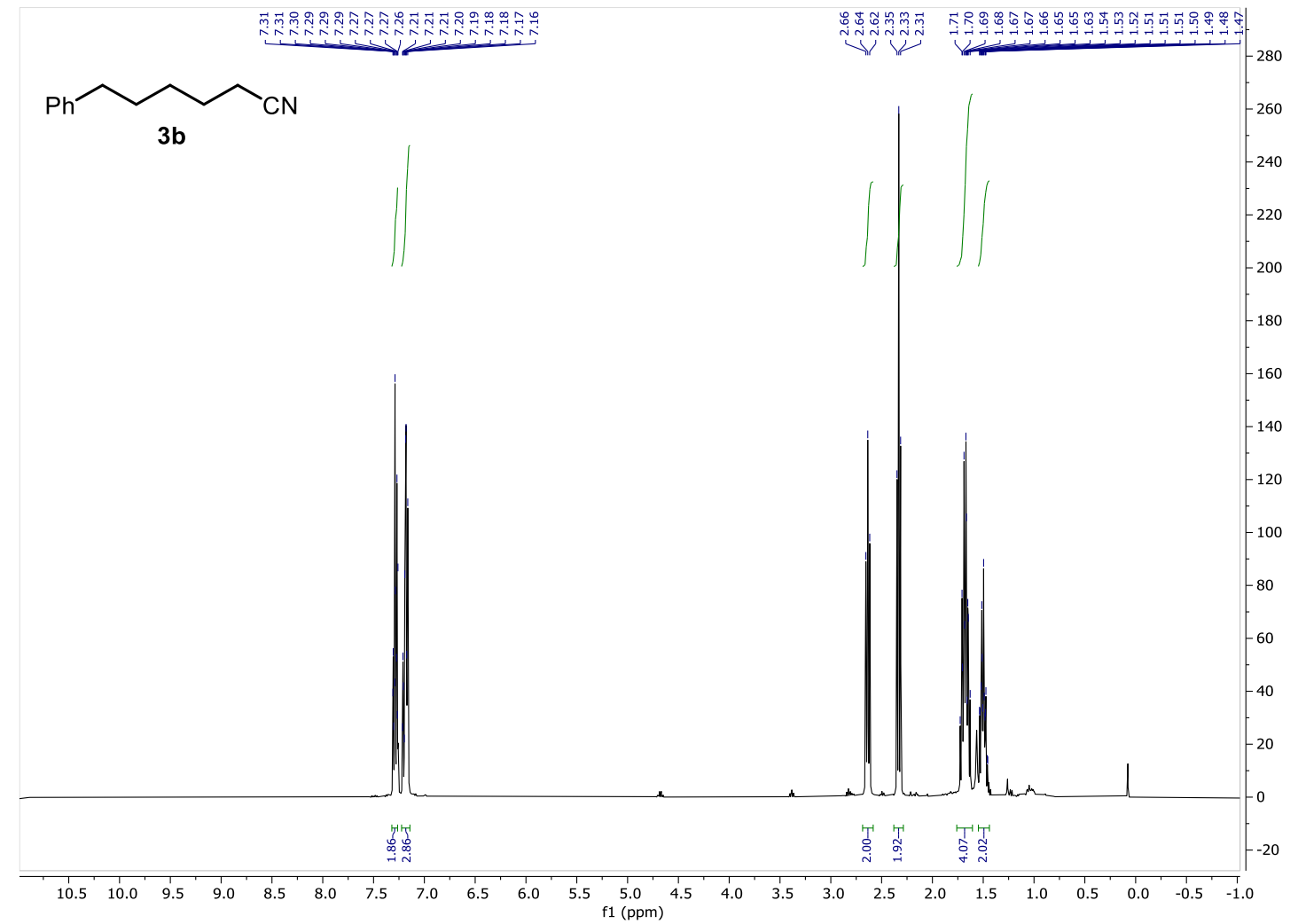

${ }^{13} \mathrm{C}$ NMR (101 MHz, $\left.\mathrm{CDCl}_{3}\right)$

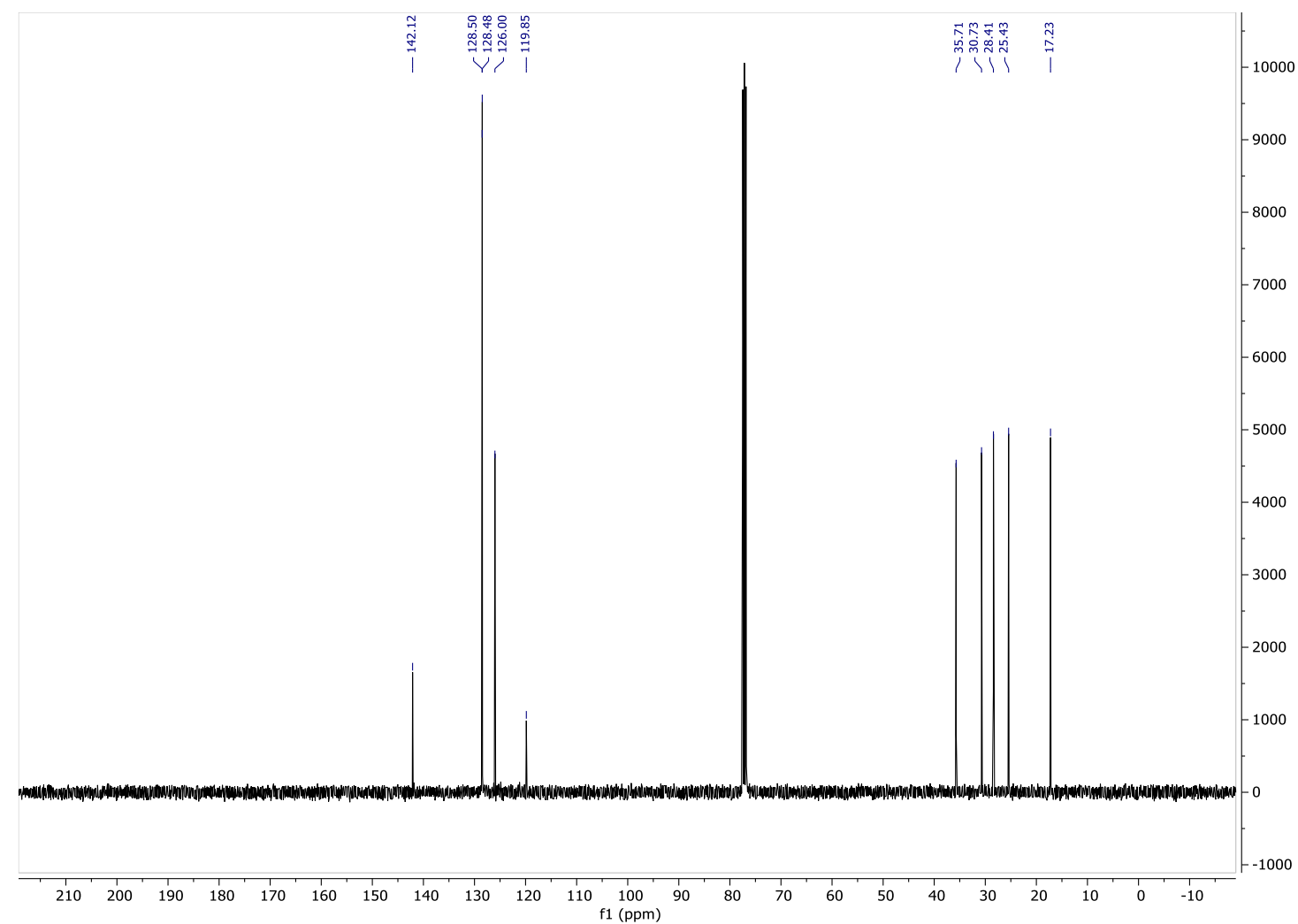


${ }^{1} \mathrm{H}$ NMR (500 MHz, $\mathrm{CDCl}_{3}$ )

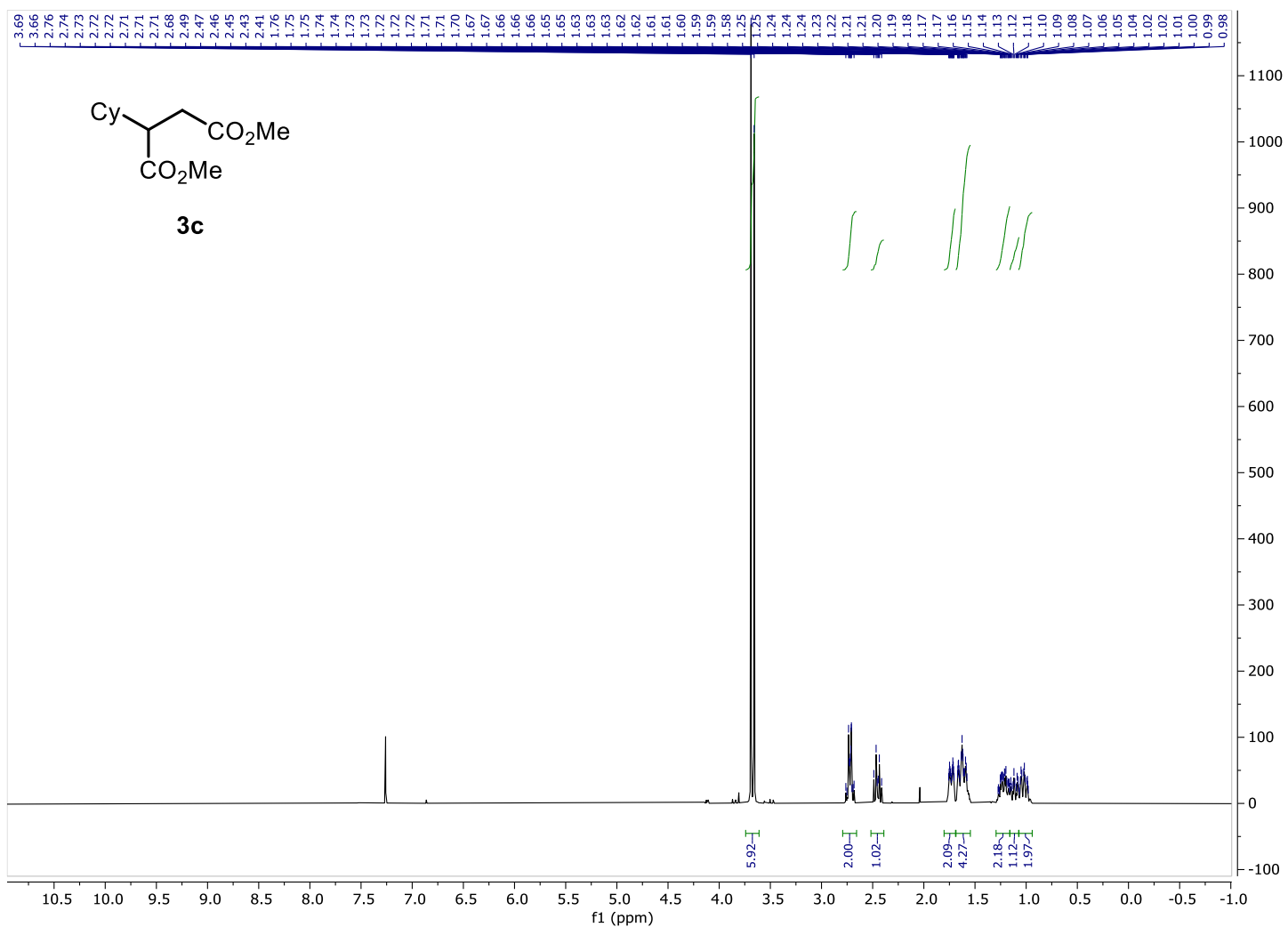

${ }^{13} \mathrm{C}$ NMR (126 MHz, $\left.\mathrm{CDCl}_{3}\right)$

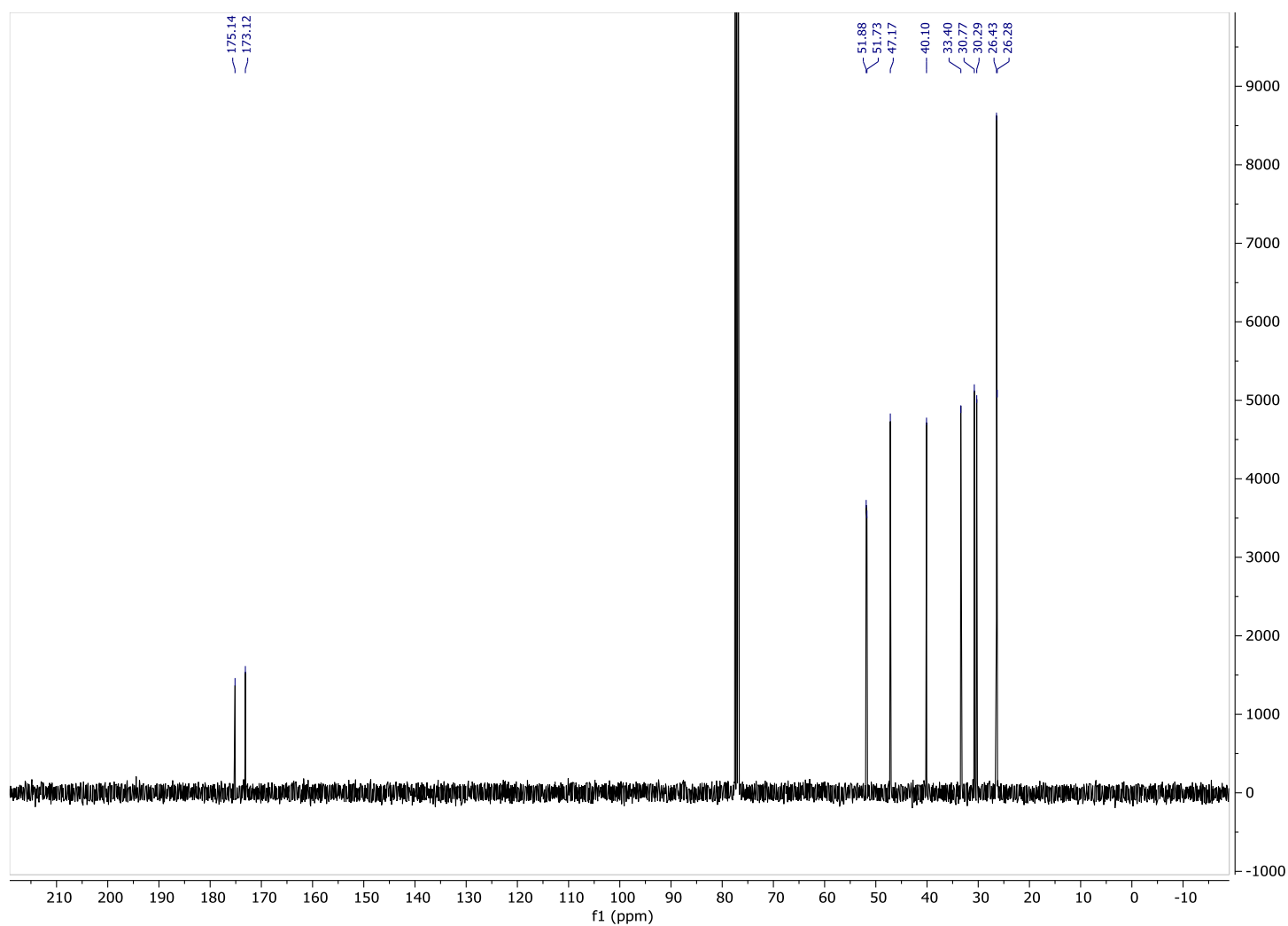


${ }^{1} \mathrm{H}$ NMR (400 MHz, $\mathrm{CDCl}_{3}$ )

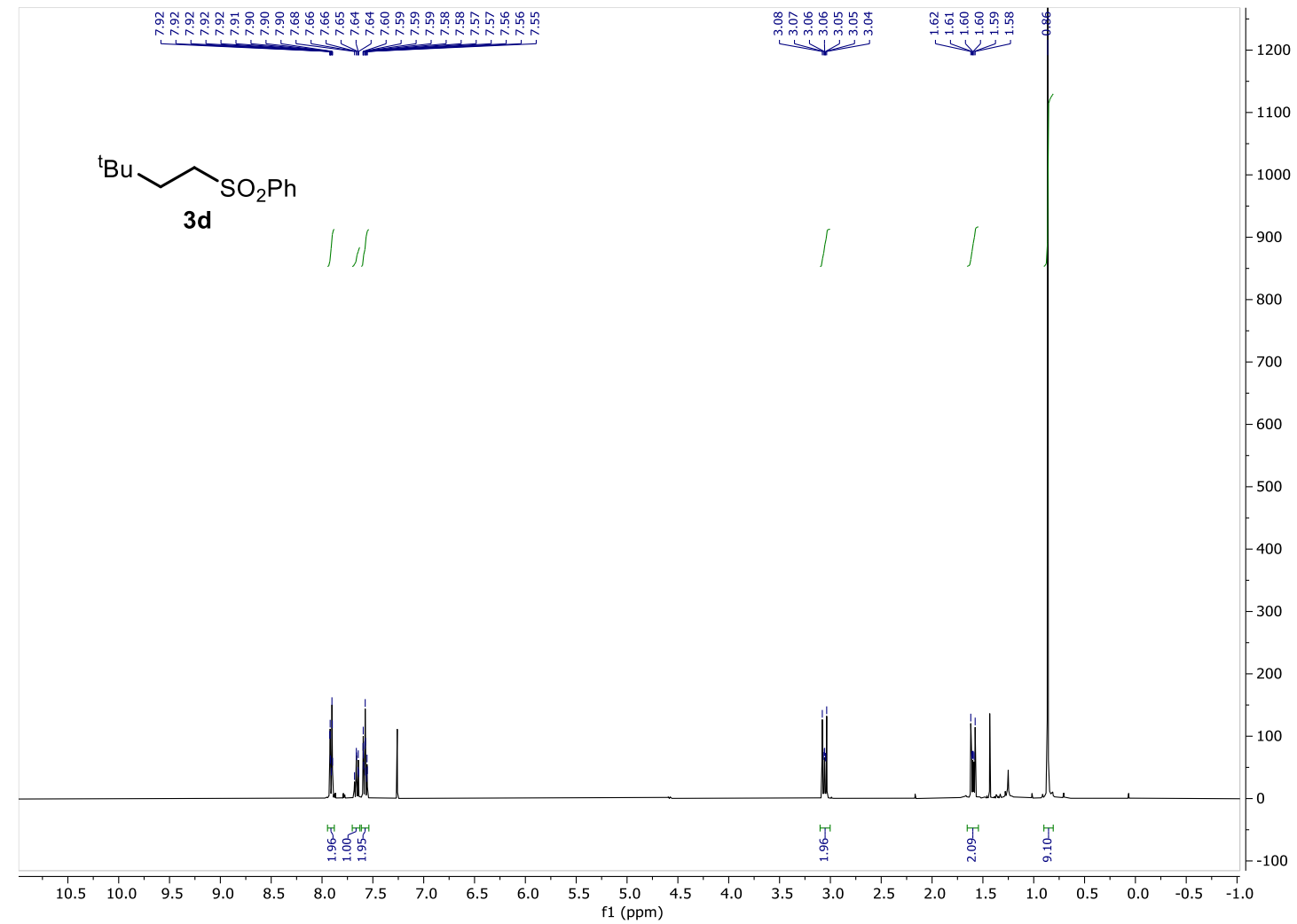

${ }^{13} \mathrm{C}$ NMR $\left(101 \mathrm{MHz}, \mathrm{CDCl}_{3}\right)$

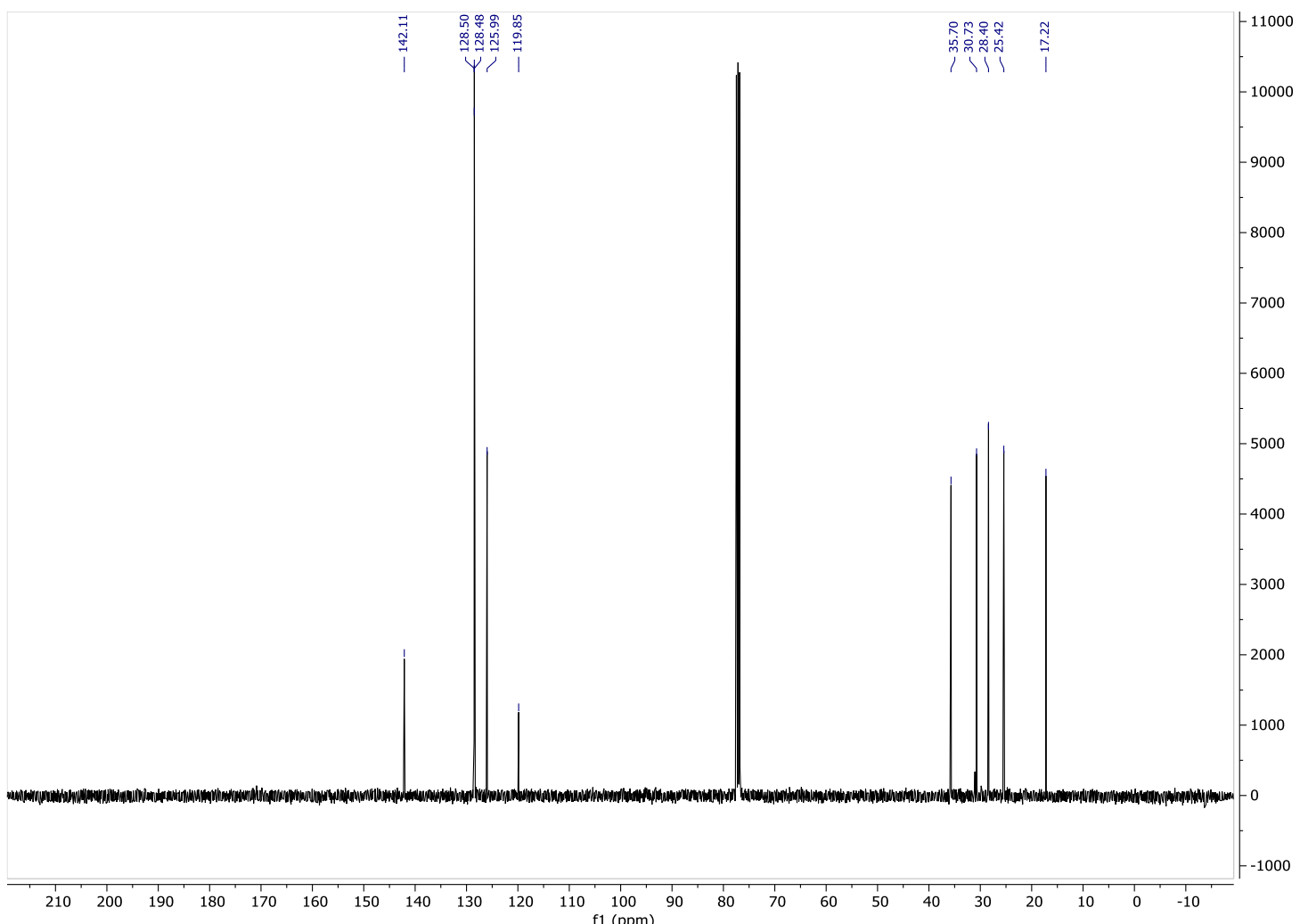


${ }^{1} \mathrm{H}$ NMR (400 MHz, $\left.\mathrm{CDCl}_{3}\right)$

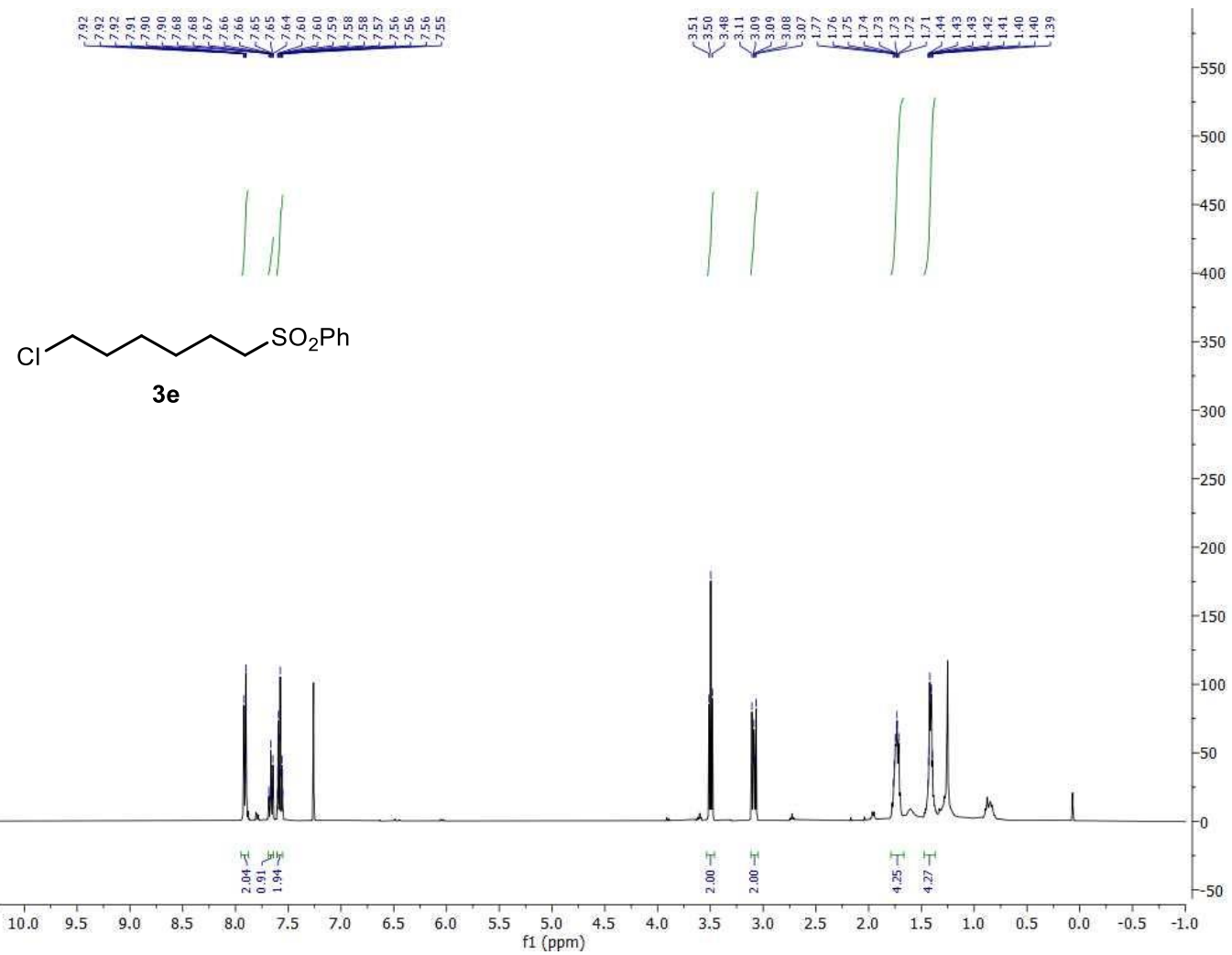

${ }^{13} \mathrm{C}$ NMR $\left(101 \mathrm{MHz}, \mathrm{CDCl}_{3}\right)$

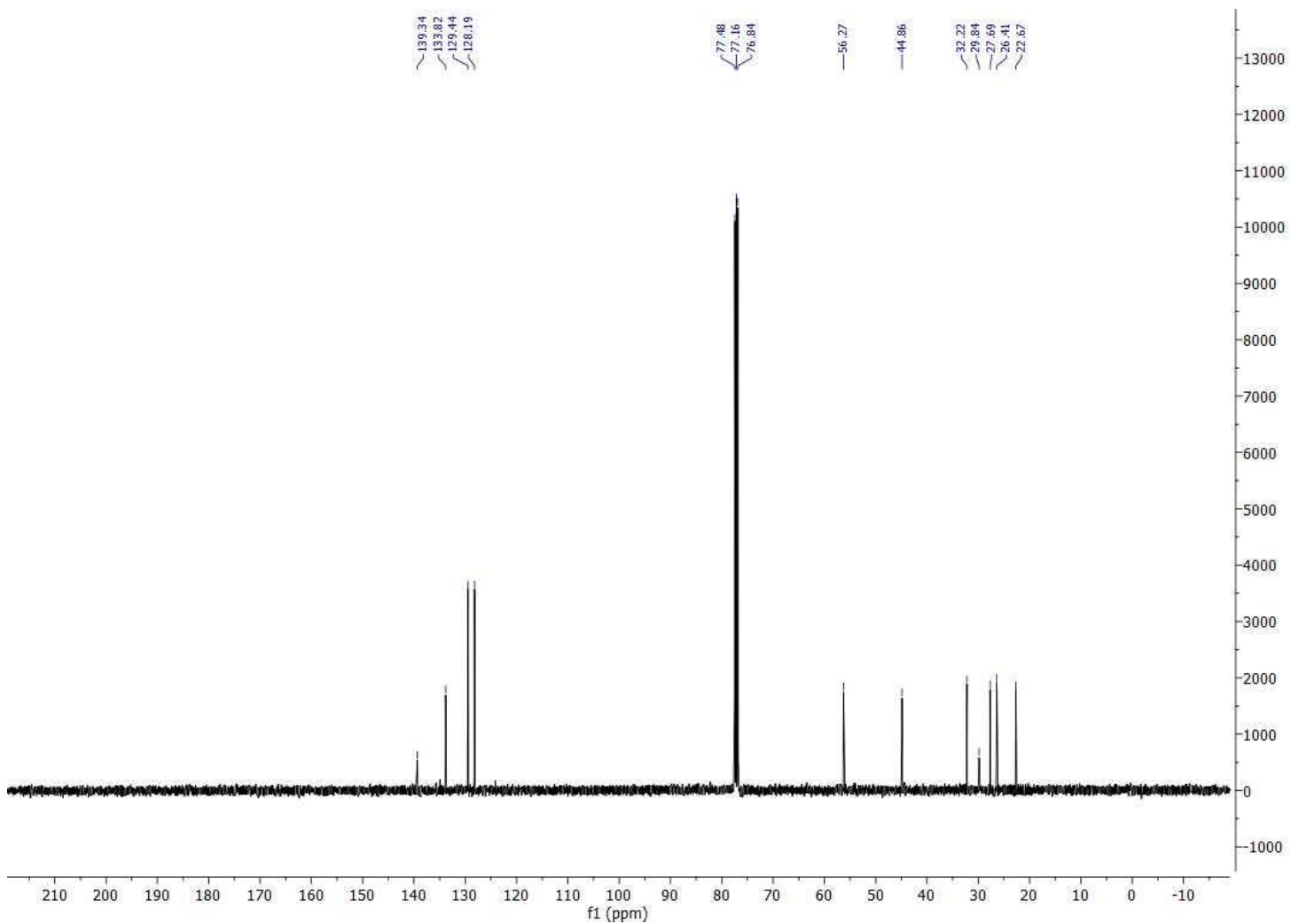


${ }^{1} \mathrm{H}$ NMR (500 MHz, $\mathrm{CDCl}_{3}$ )

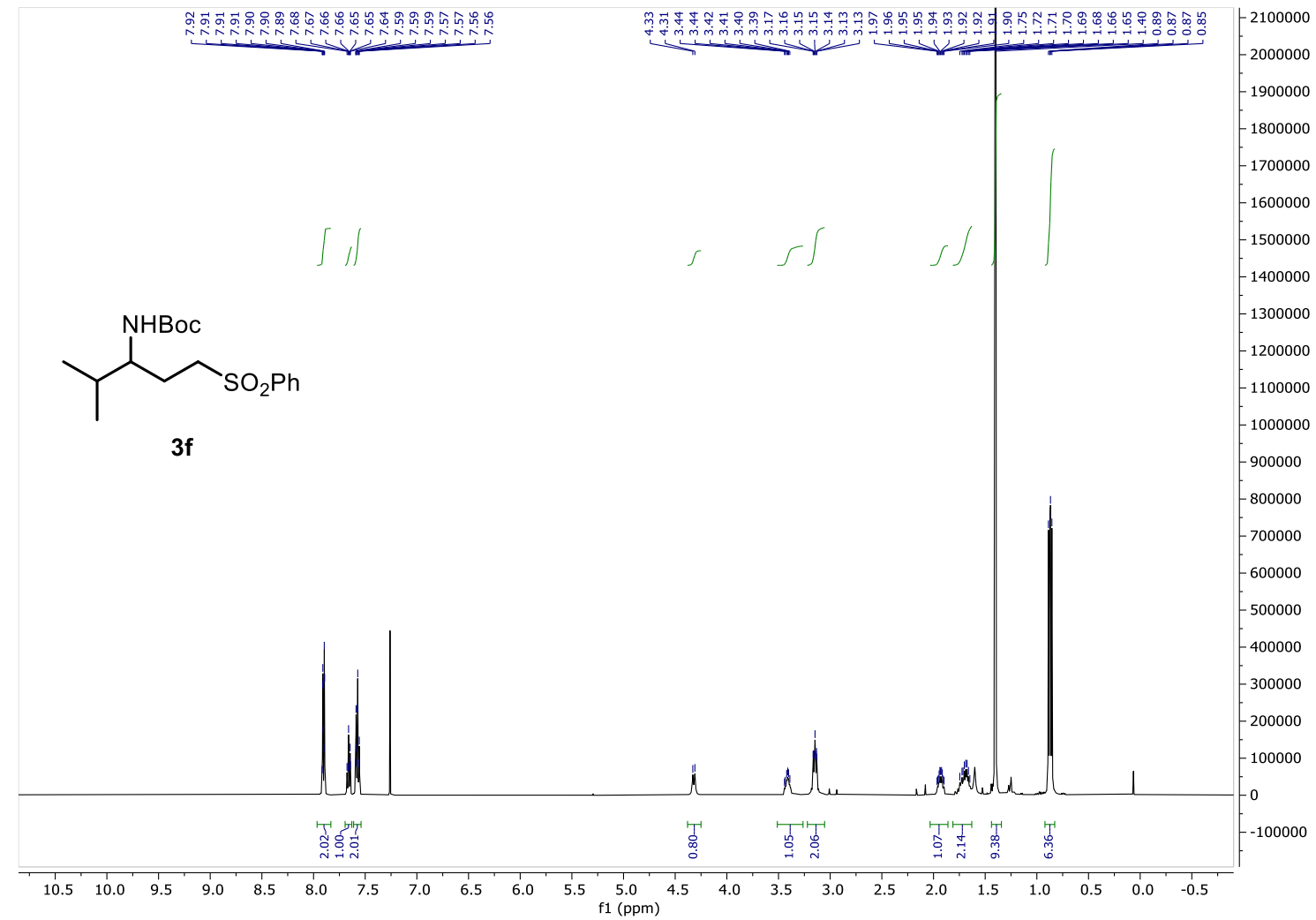

${ }^{13} \mathrm{C}$ NMR (126 MHz, $\left.\mathrm{CDCl}_{3}\right)$

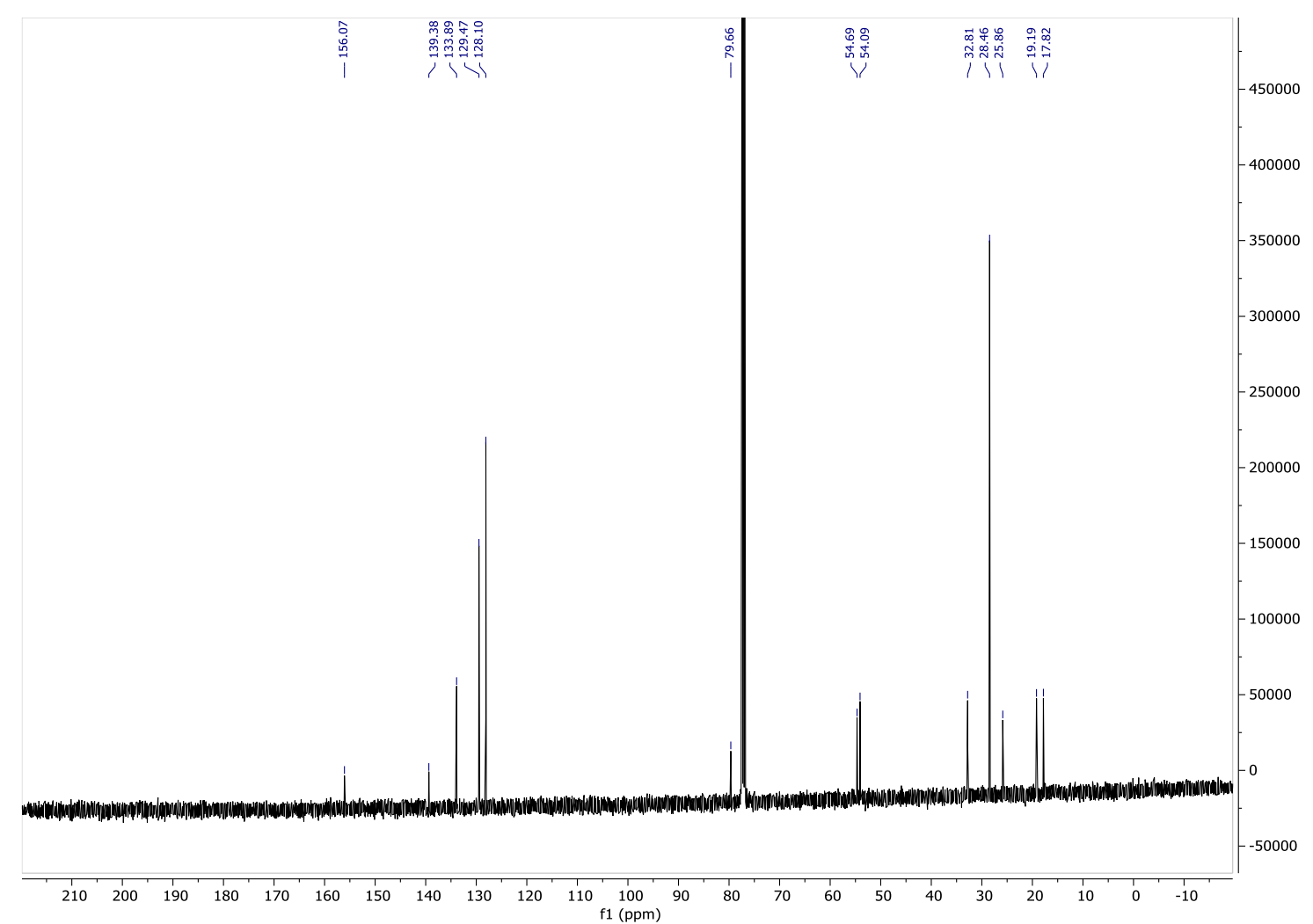


${ }^{1} \mathrm{H}$ NMR (400 MHz, $\mathrm{CDCl}_{3}$ )

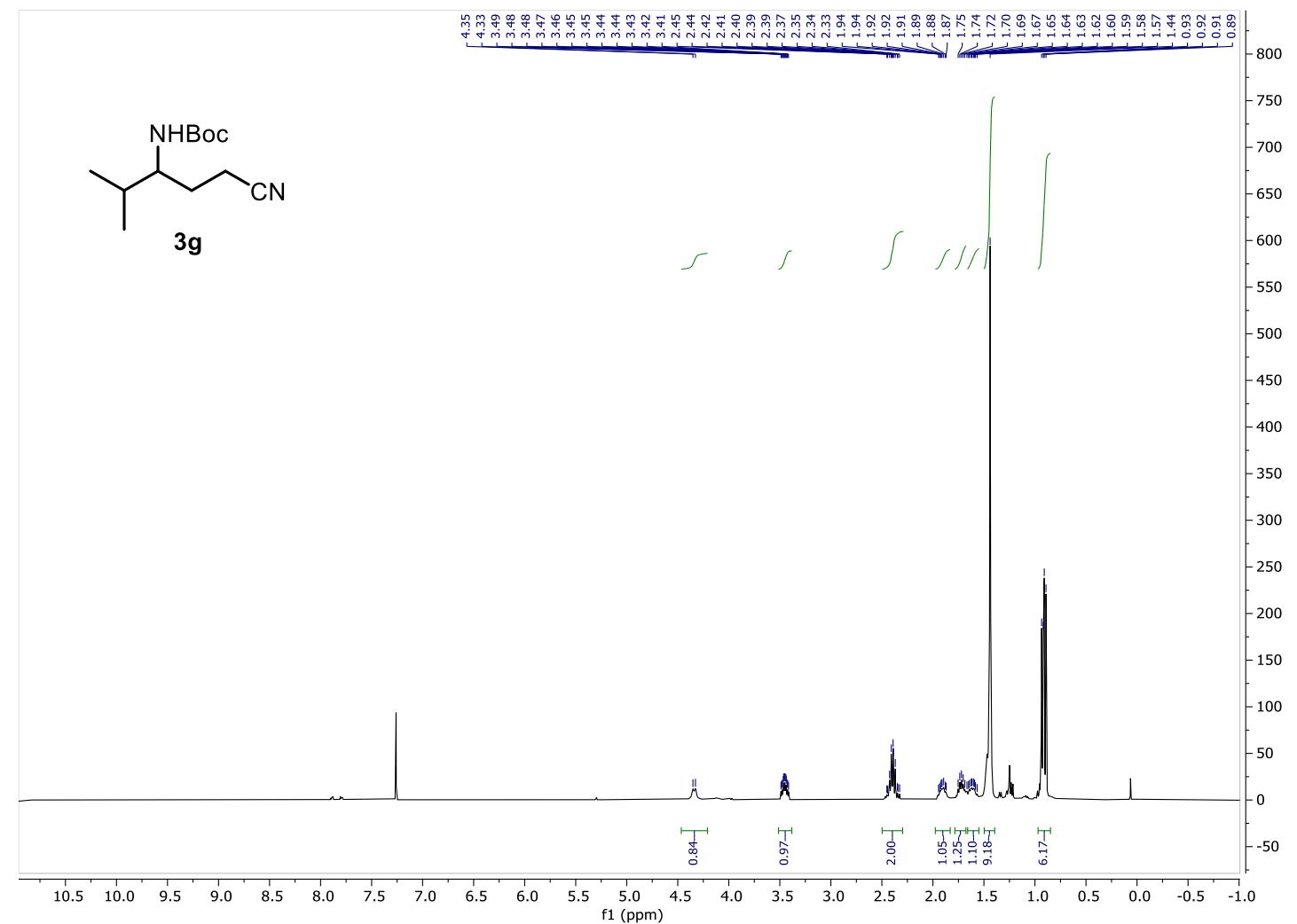

${ }^{13} \mathrm{C}$ NMR $\left(101 \mathrm{MHz}, \mathrm{CDCl}_{3}\right)$

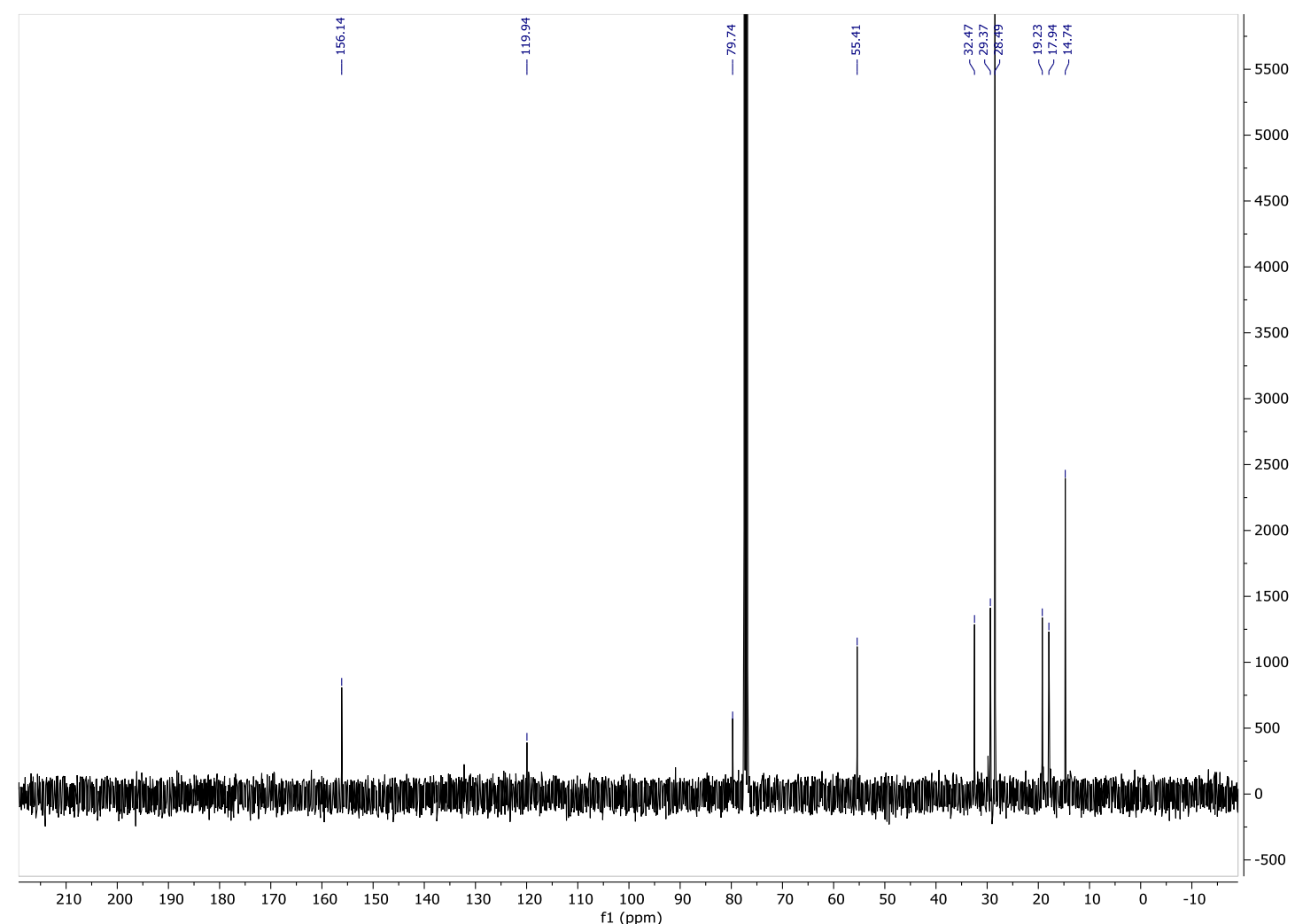


${ }^{1} \mathrm{H}$ NMR (400 MHz, $\mathrm{CDCl}_{3}$ )

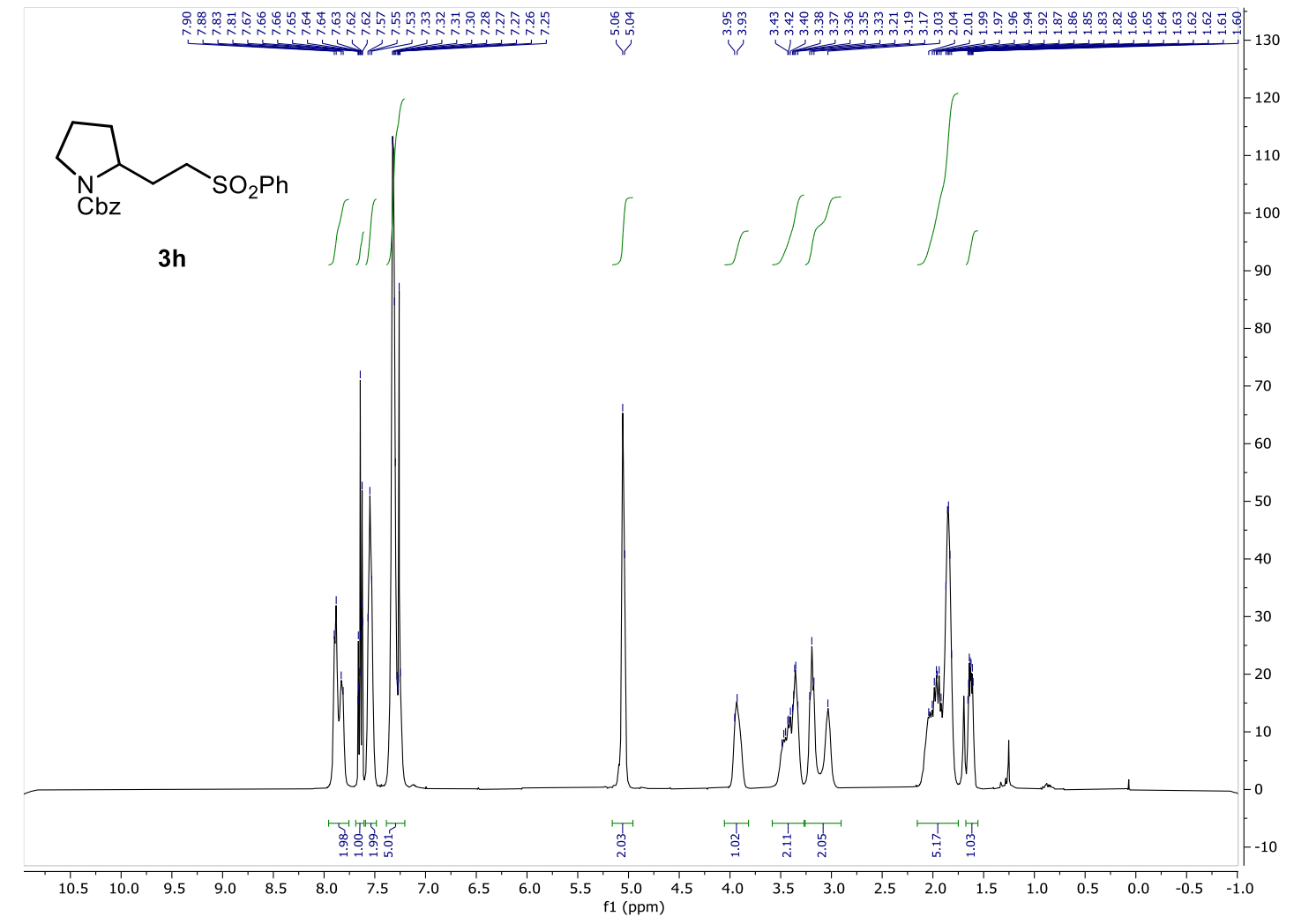

${ }^{13} \mathrm{C}$ NMR $\left(101 \mathrm{MHz}, \mathrm{CDCl}_{3}\right)$

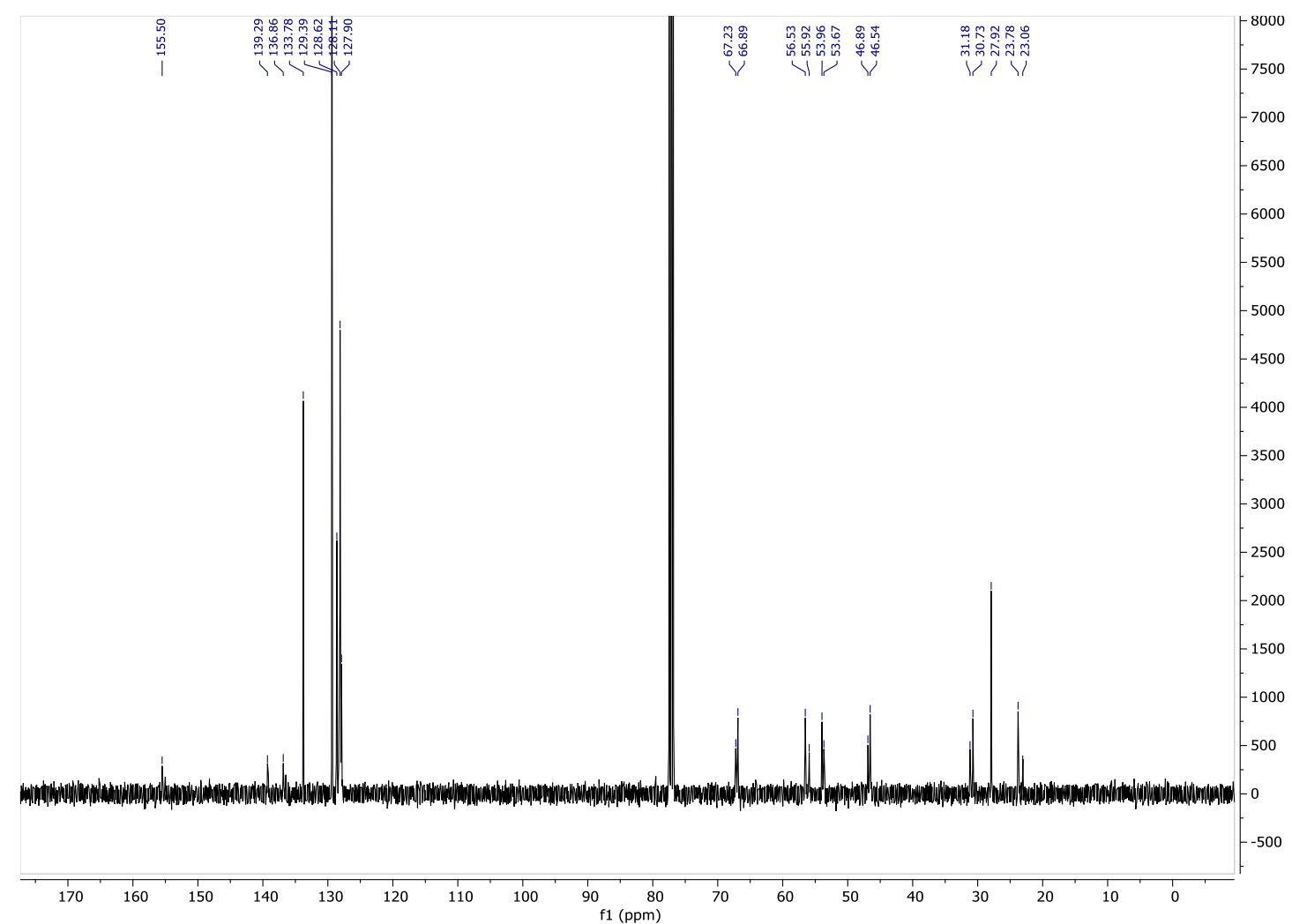


${ }^{1} \mathrm{H}$ NMR (400 MHz, $\mathrm{CDCl}_{3}$ )

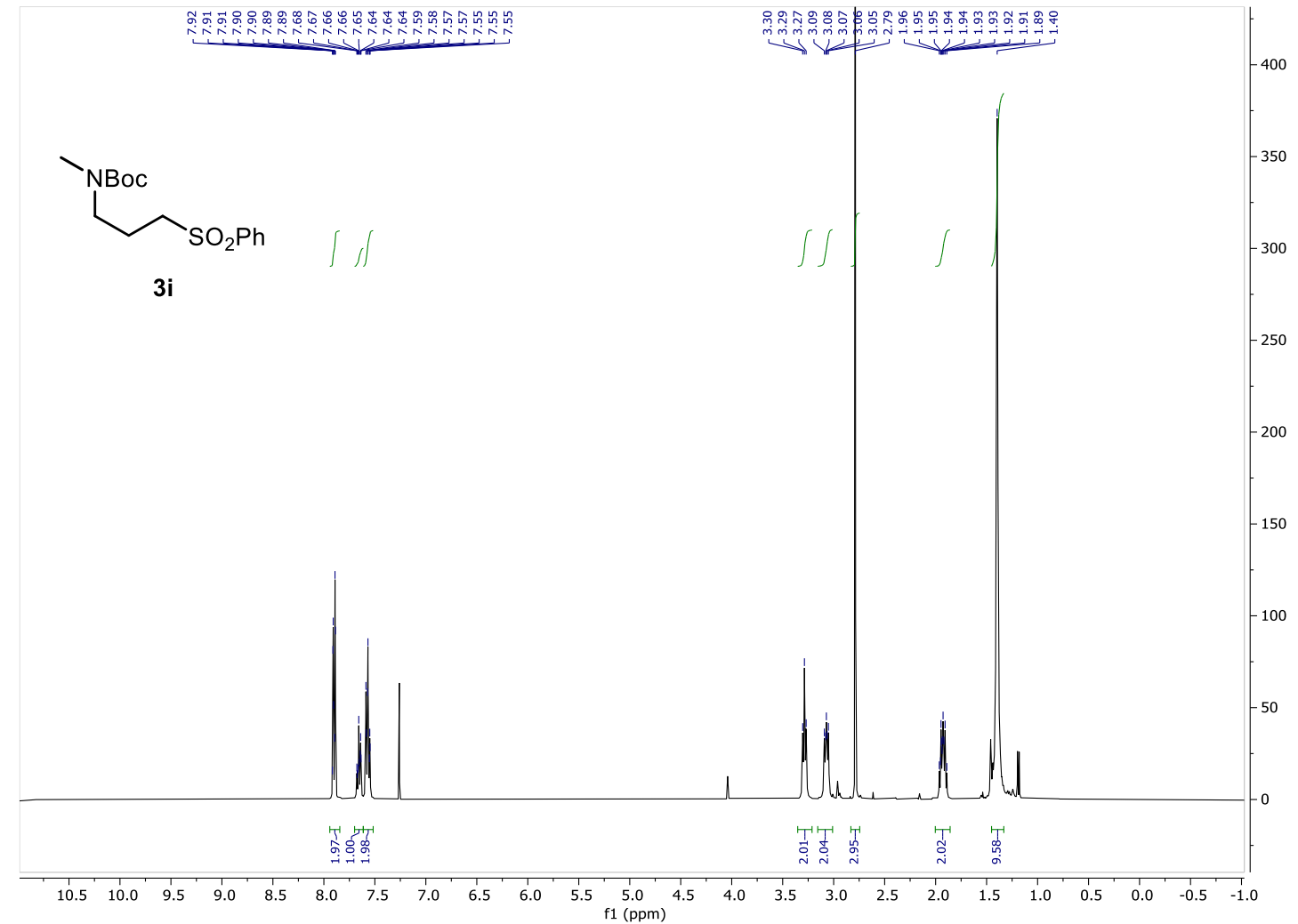

${ }^{13} \mathrm{C}$ NMR $\left(101 \mathrm{MHz}, \mathrm{CDCl}_{3}\right)$

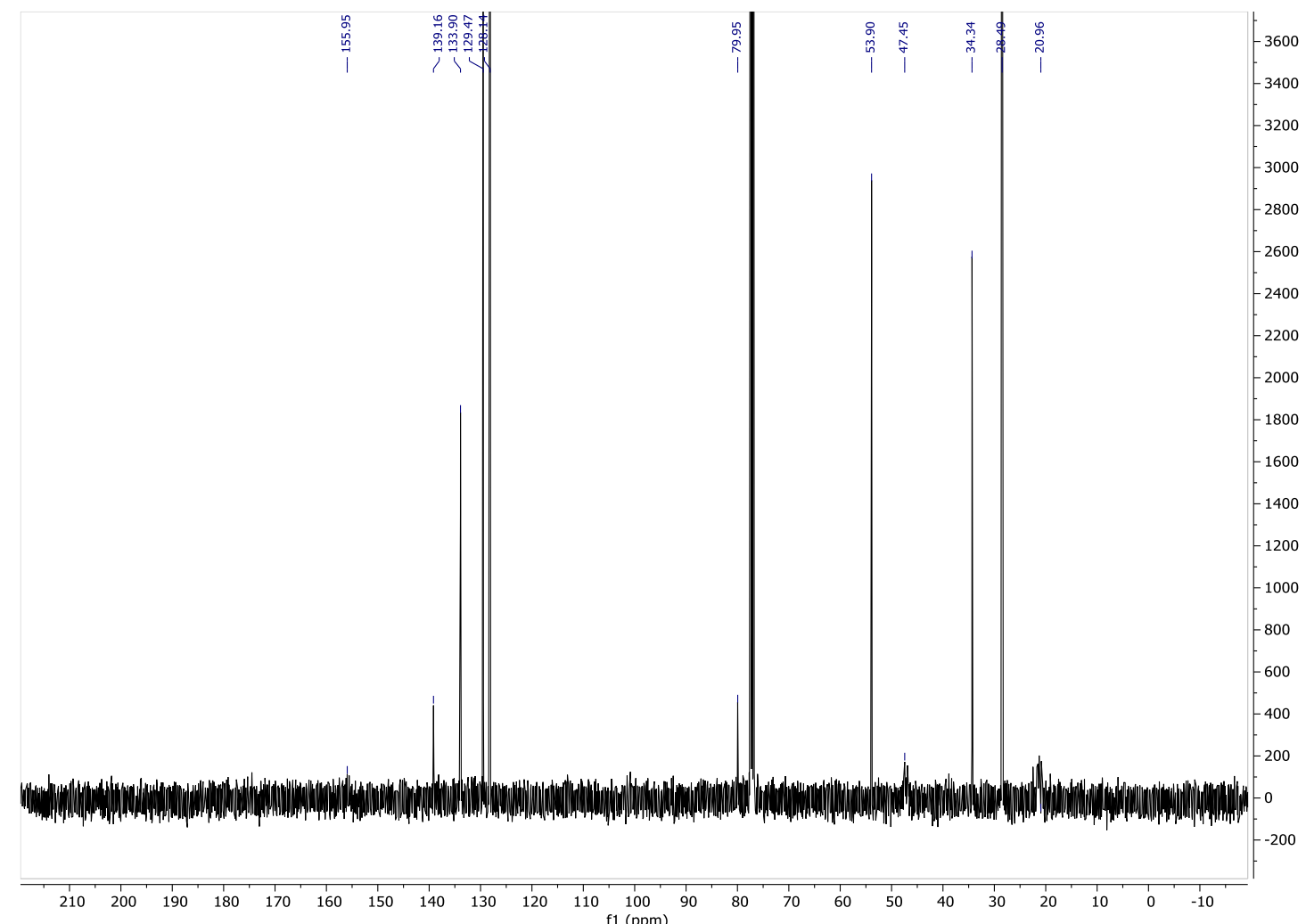


${ }^{1} \mathrm{H}$ NMR (400 MHz, $\mathrm{CDCl}_{3}$ )

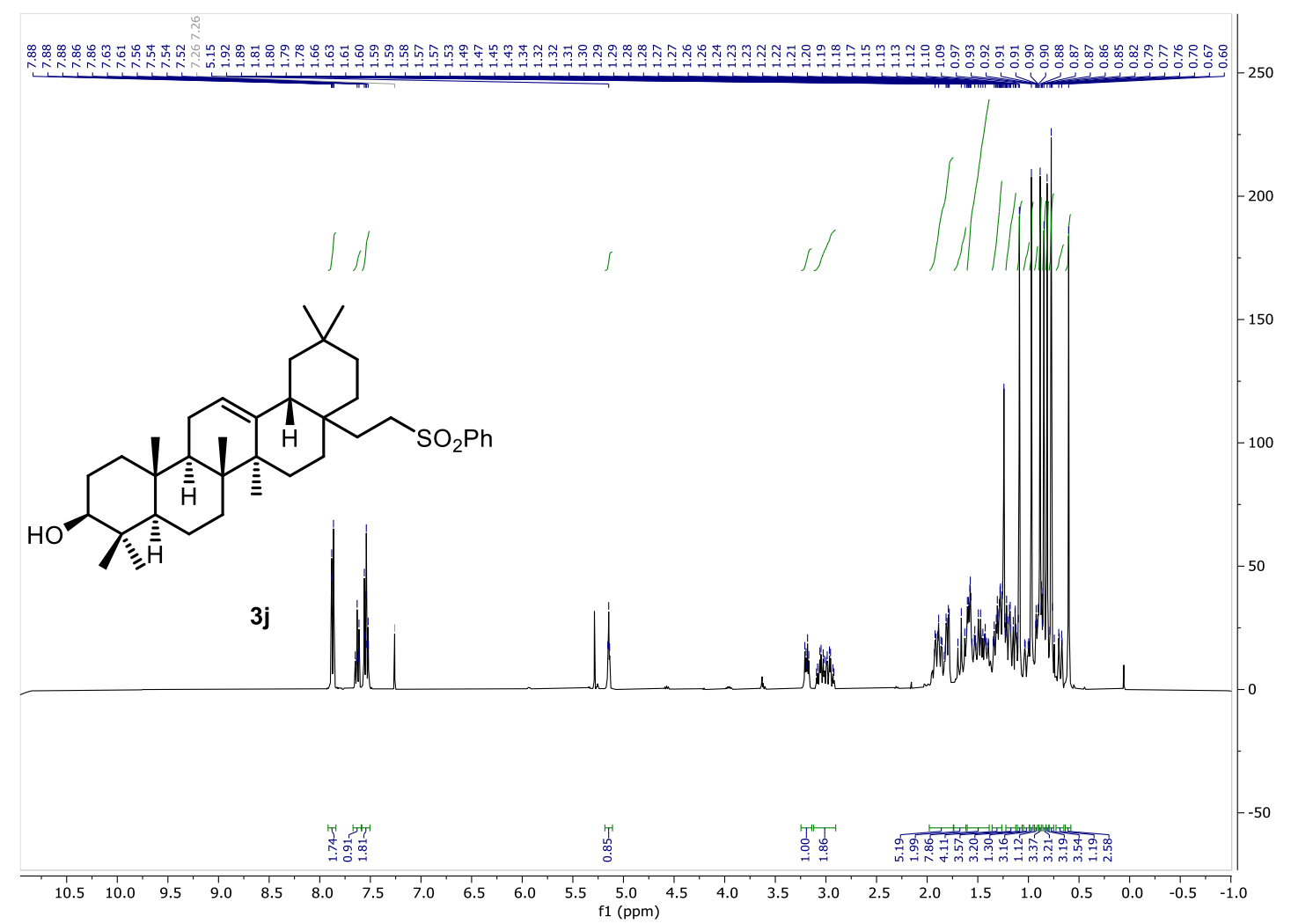

${ }^{13} \mathrm{C}$ NMR (101 MHz, $\left.\mathrm{CDCl}_{3}\right)$

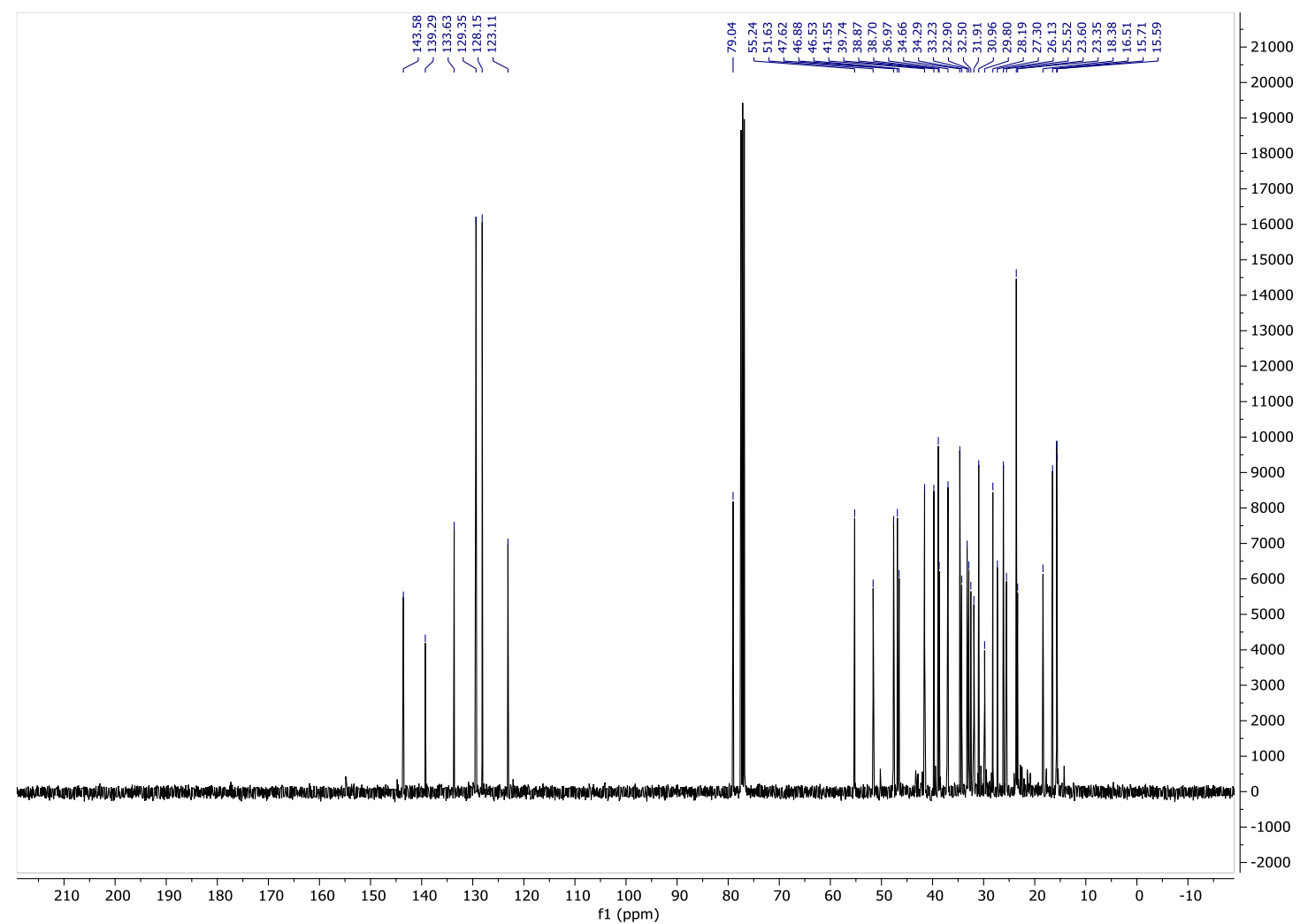


${ }^{1} \mathrm{H}$ NMR (400 MHz, $\mathrm{CDCl}_{3}$ )

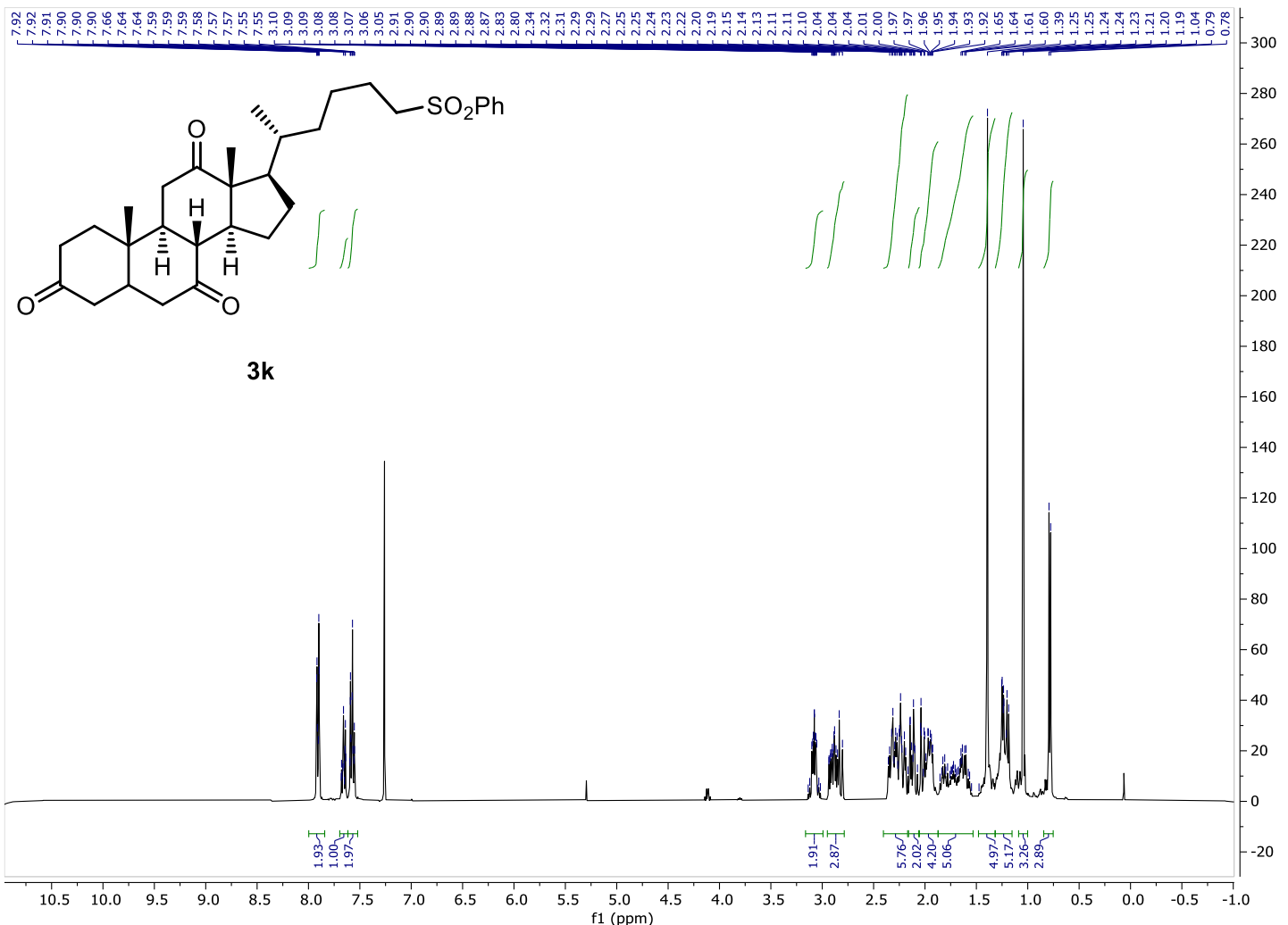

${ }^{13} \mathrm{C}$ NMR $\left(101 \mathrm{MHz}, \mathrm{CDCl}_{3}\right)$

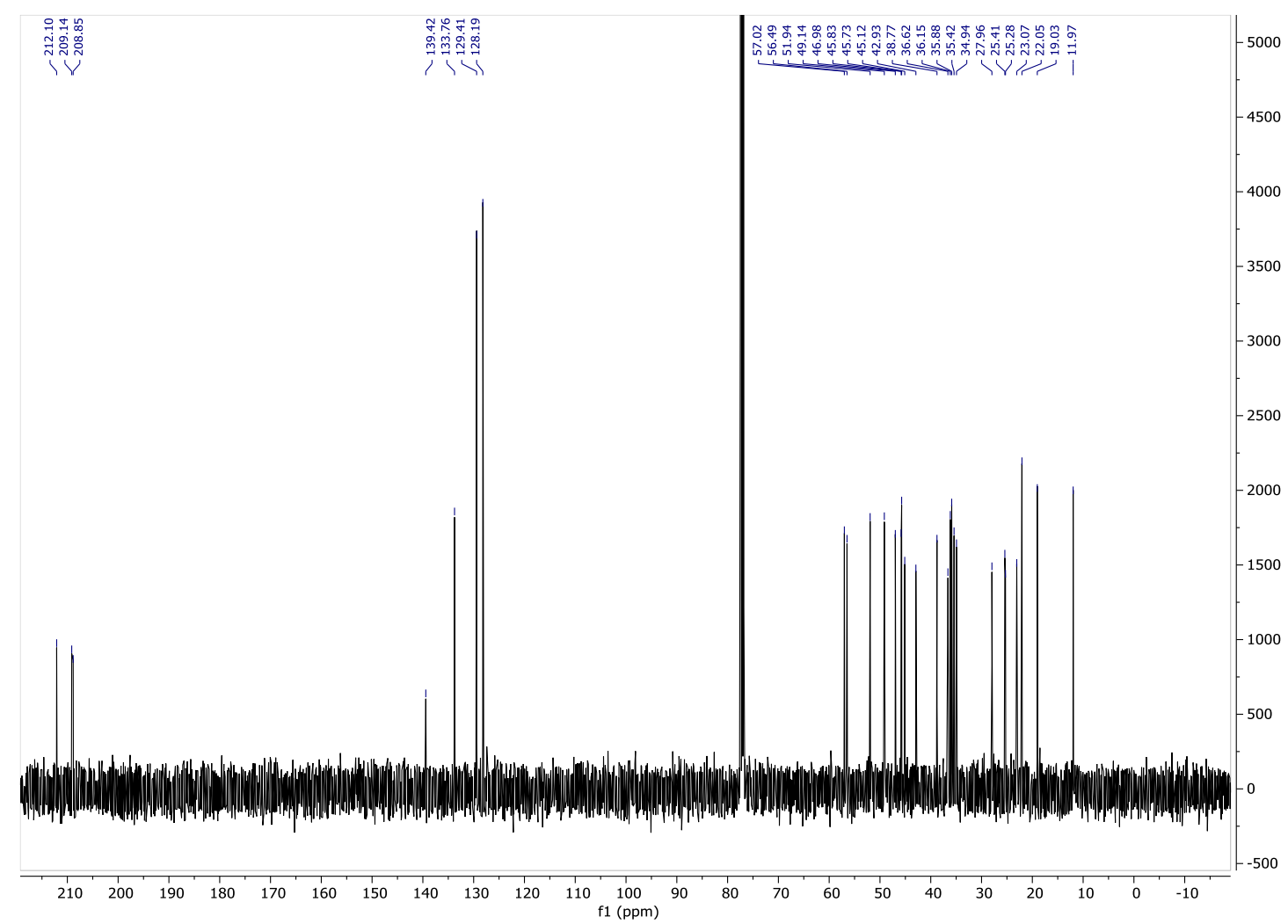


${ }^{1} \mathrm{H}$ NMR (500 MHz, Methanol- $d_{4}$ )

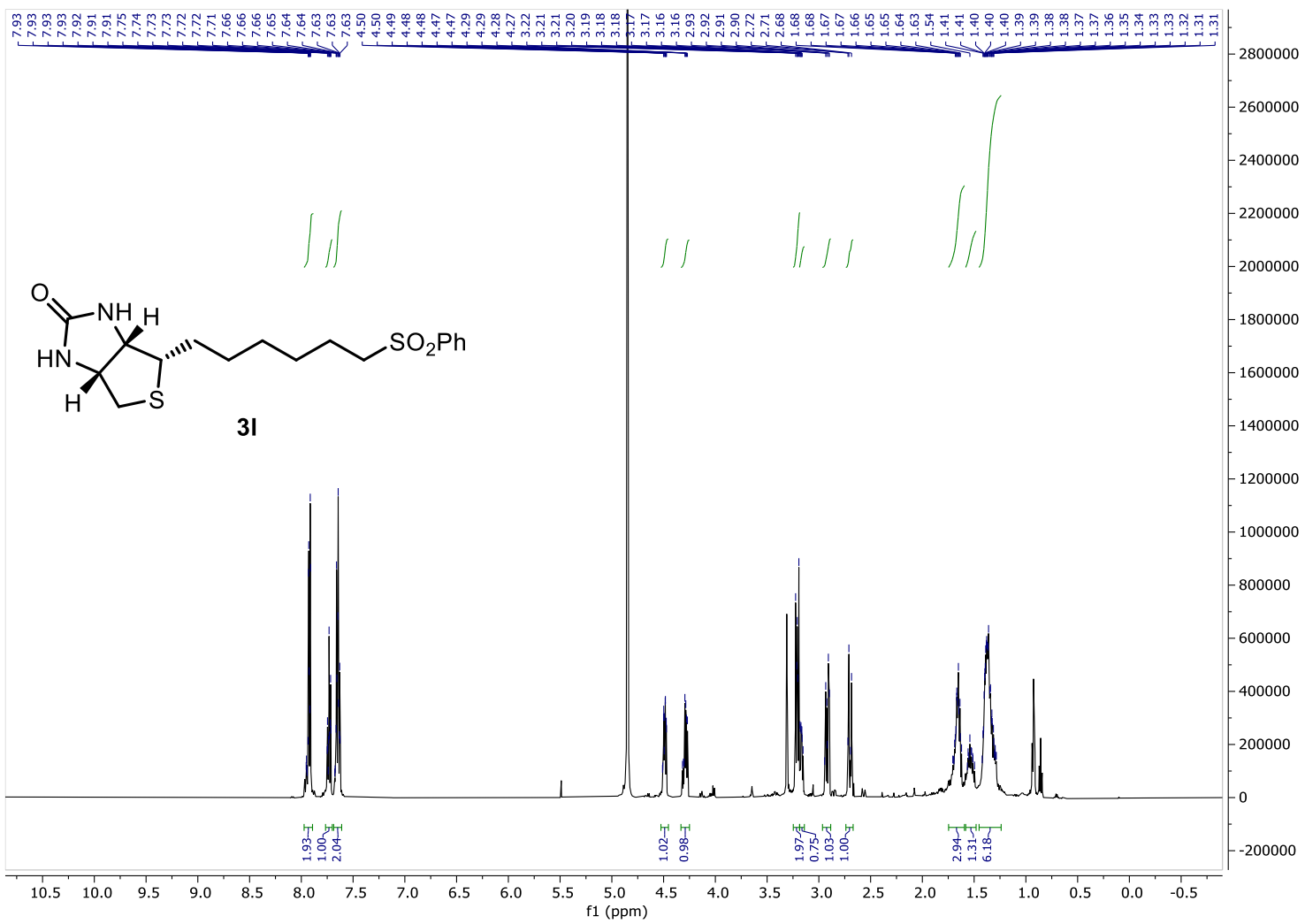

${ }^{13} \mathrm{C}$ NMR $\left(101 \mathrm{MHz}, \mathrm{CDCl}_{3}\right)$

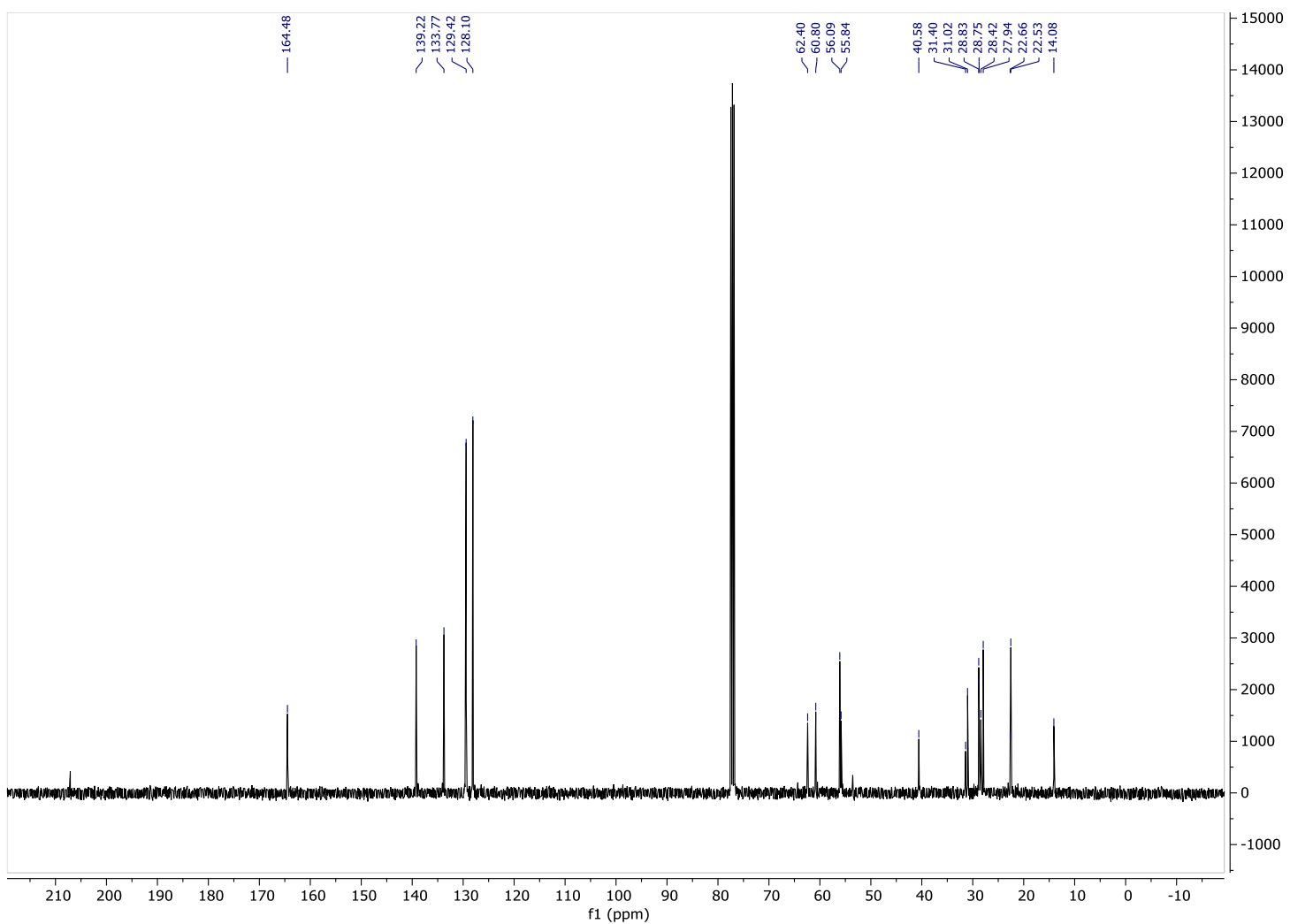


${ }^{1} \mathrm{H}$ NMR (400 MHz, $\mathrm{CDCl}_{3}$ )

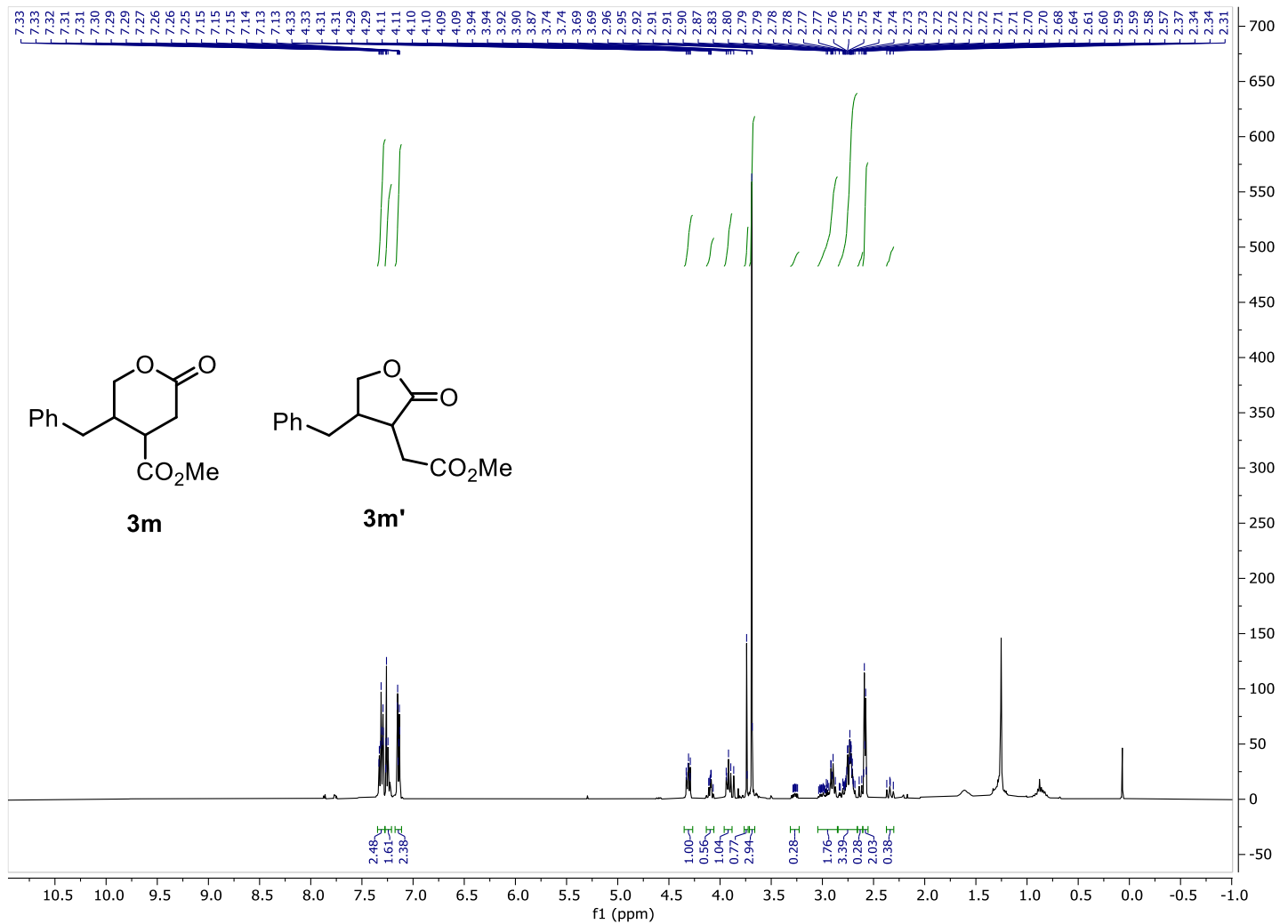

${ }^{13} \mathrm{C}$ NMR (101 MHz, $\mathrm{CDCl}_{3}$ )

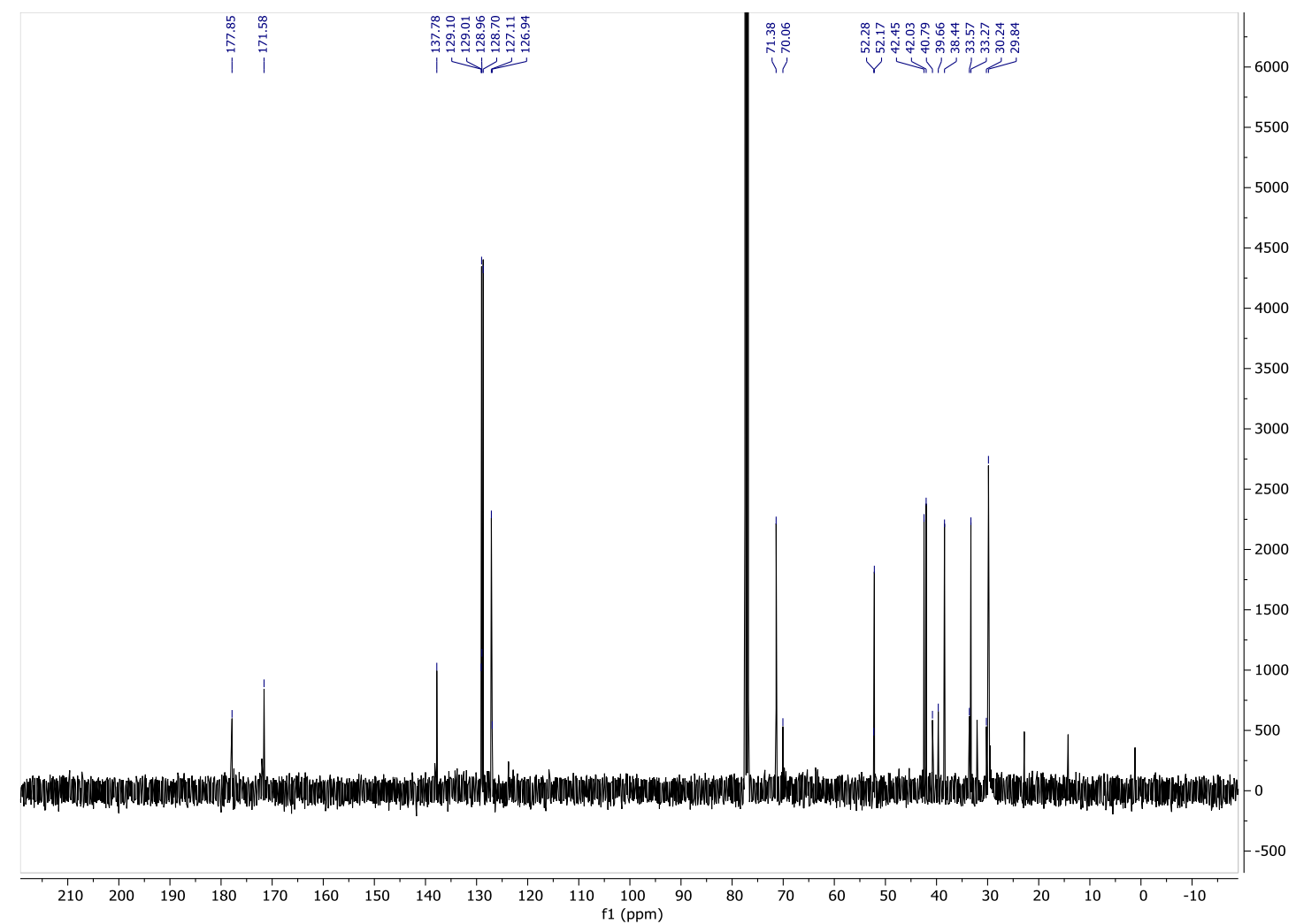


${ }^{1} \mathrm{H}$ NMR (400 MHz, $\mathrm{CDCl}_{3}$ )

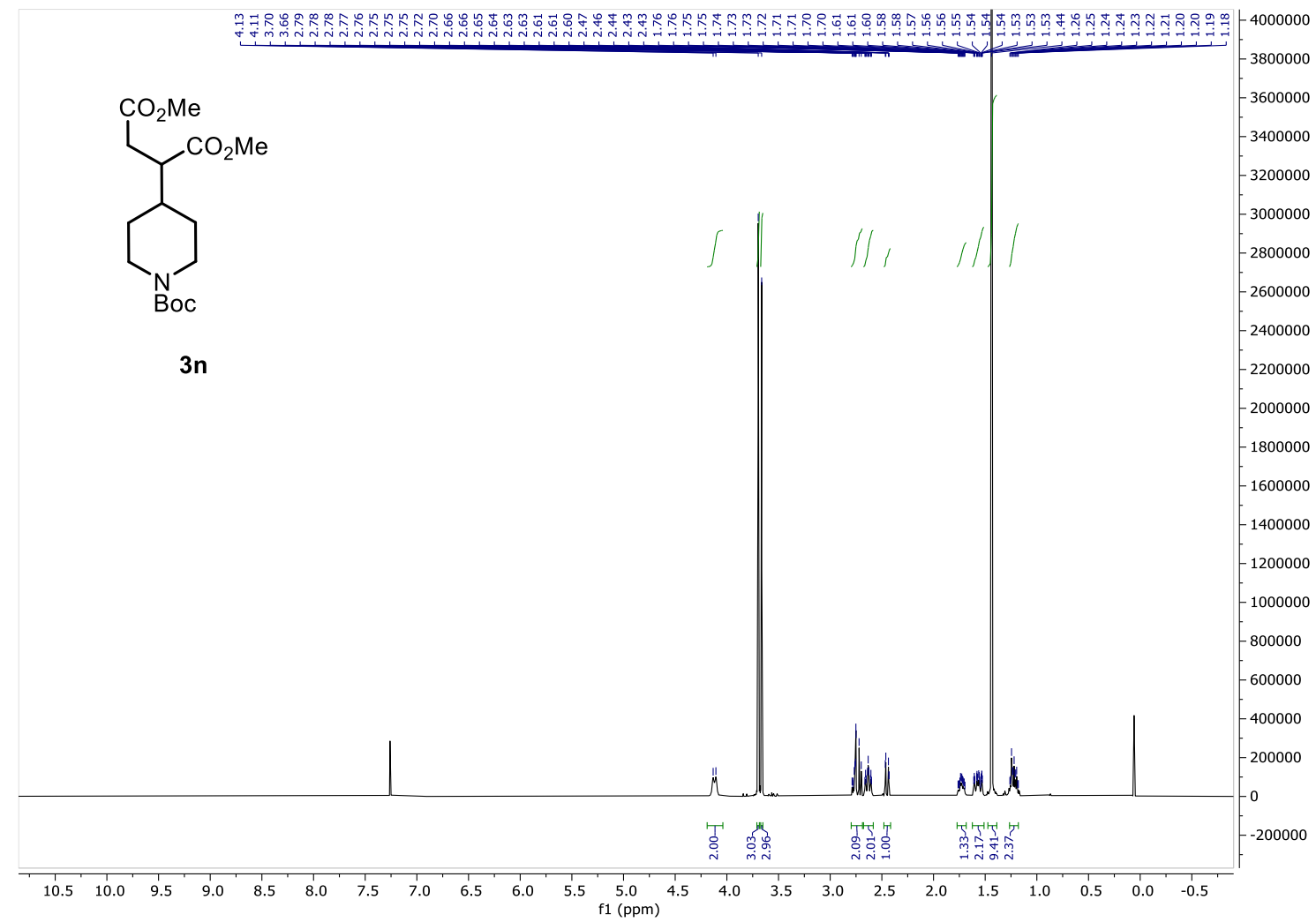

${ }^{13} \mathrm{C}$ NMR (126 MHz, $\left.\mathrm{CDCl}_{3}\right)$

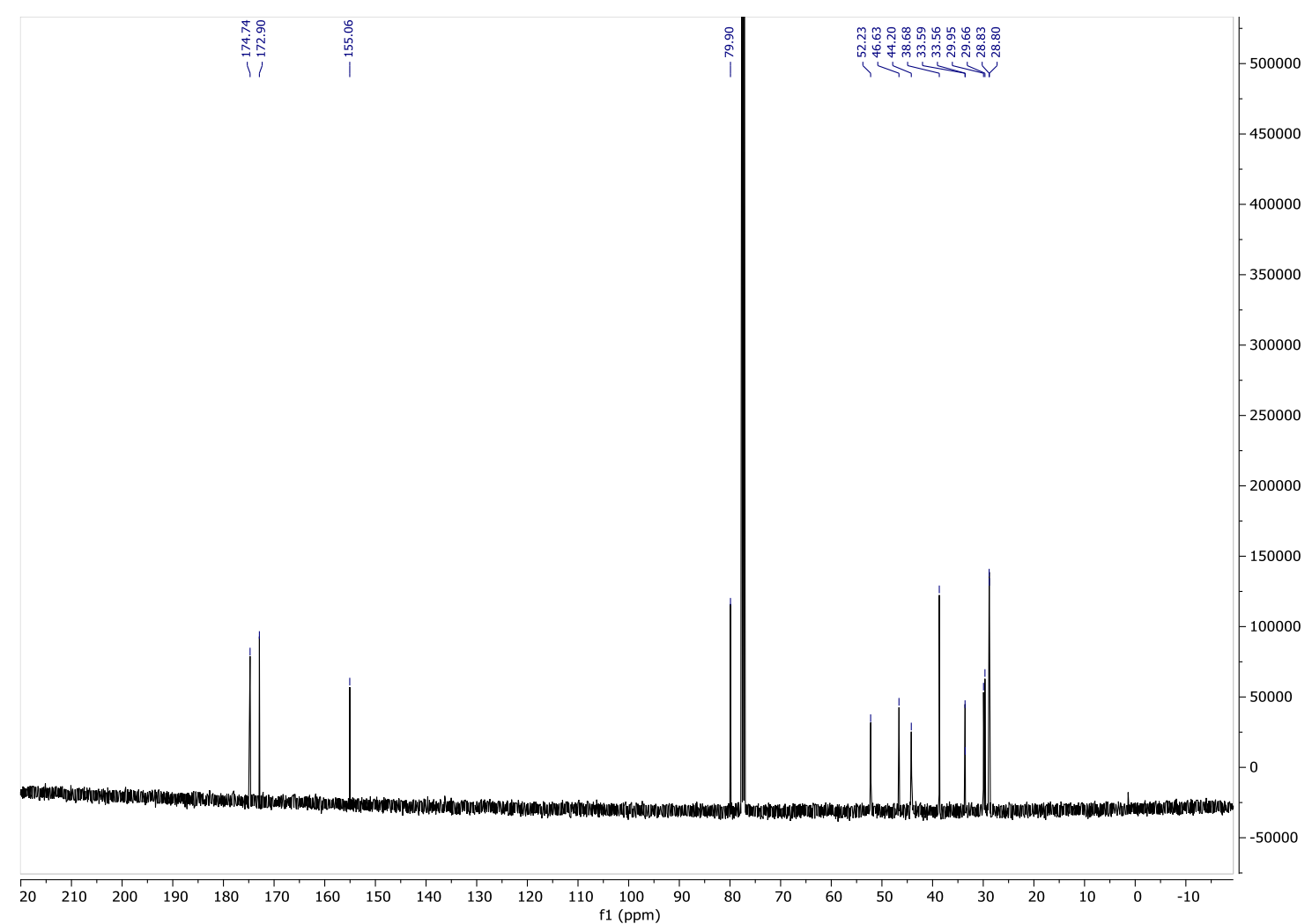


${ }^{1} \mathrm{H}$ NMR (400 MHz, $\mathrm{CDCl}_{3}$ )

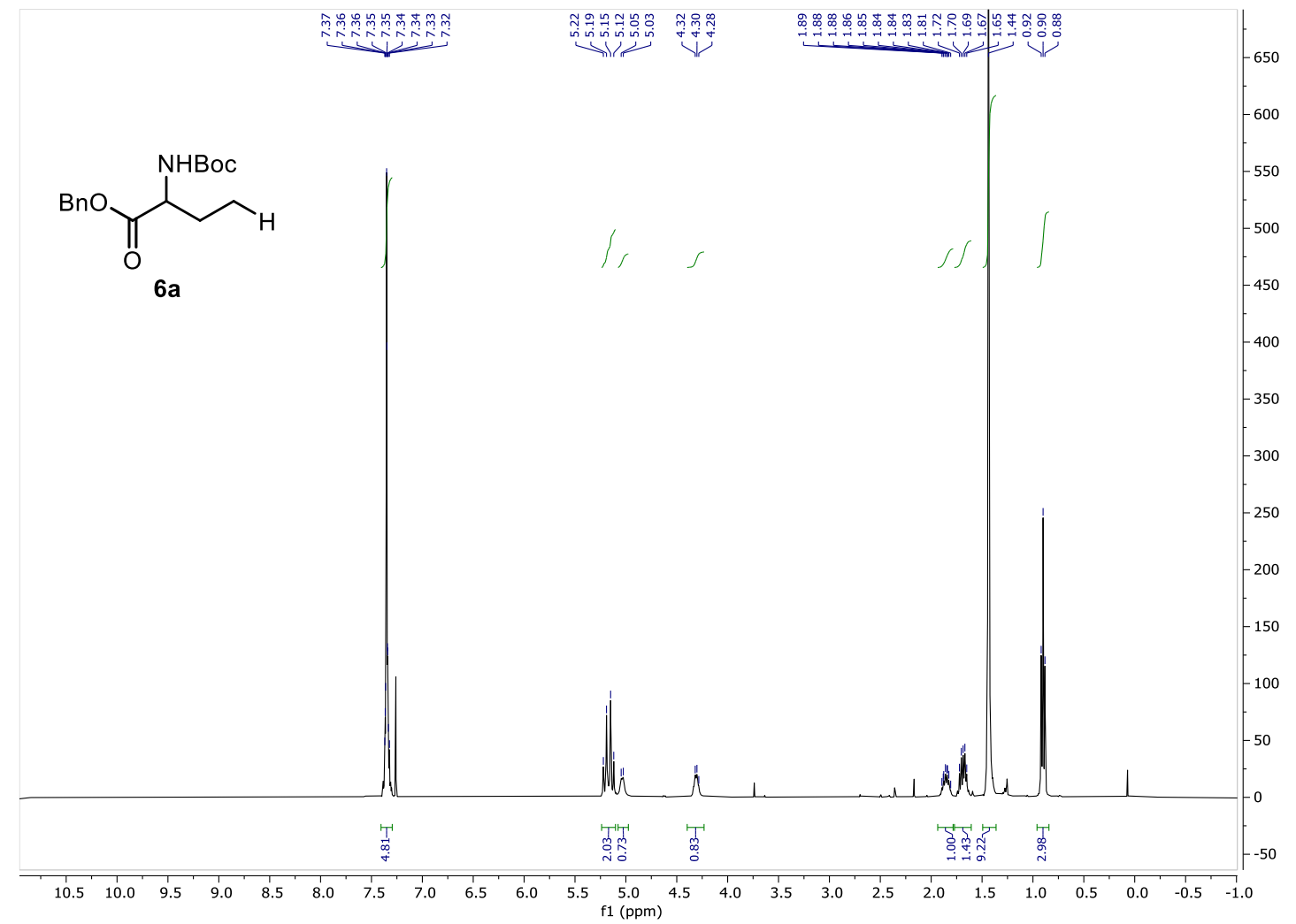

${ }^{13} \mathrm{C}$ NMR $\left(126 \mathrm{MHz}, \mathrm{CDCl}_{3}\right)$

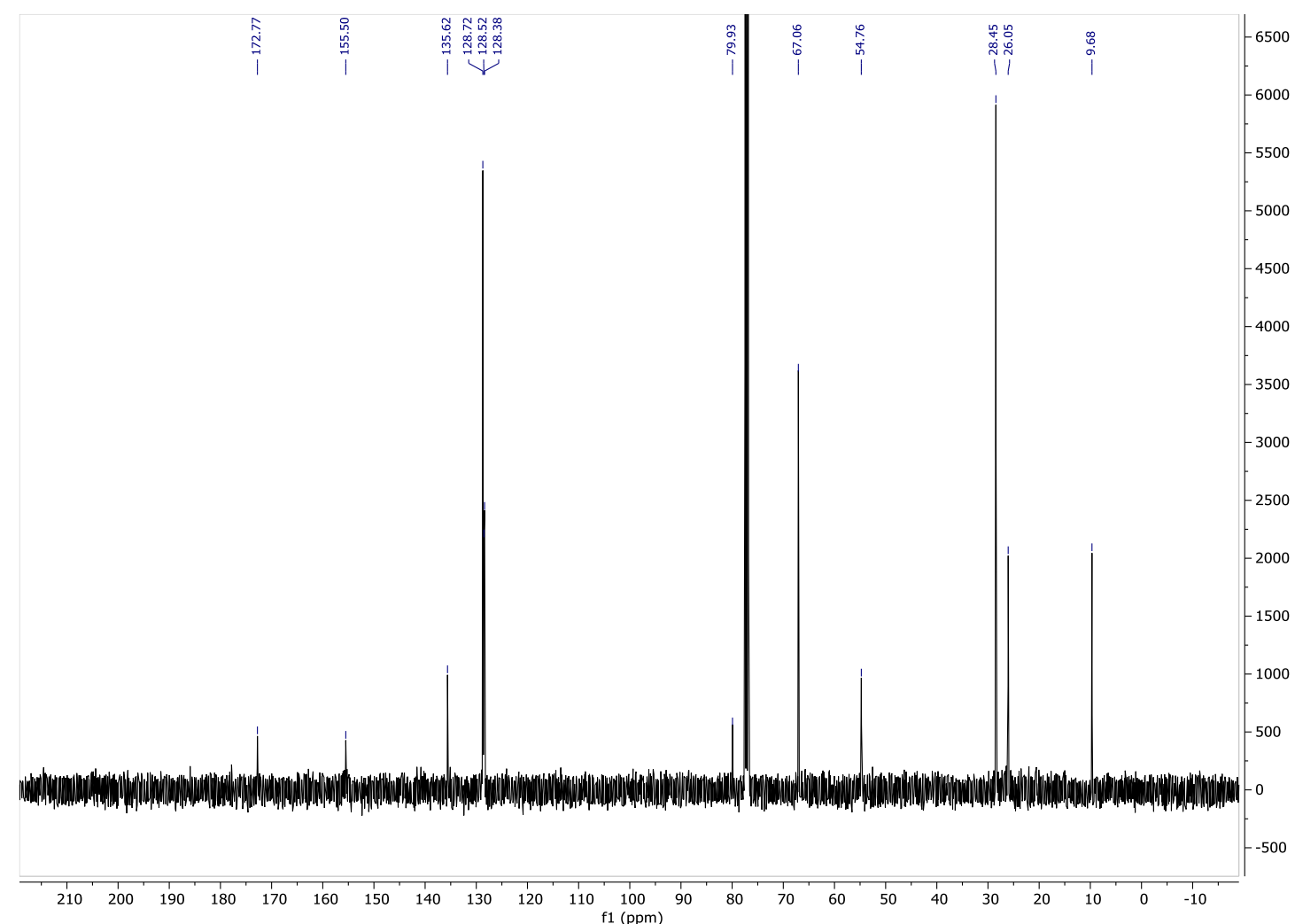


${ }^{1} \mathrm{H}$ NMR (500 MHz, $\mathrm{CDCl}_{3}$ )

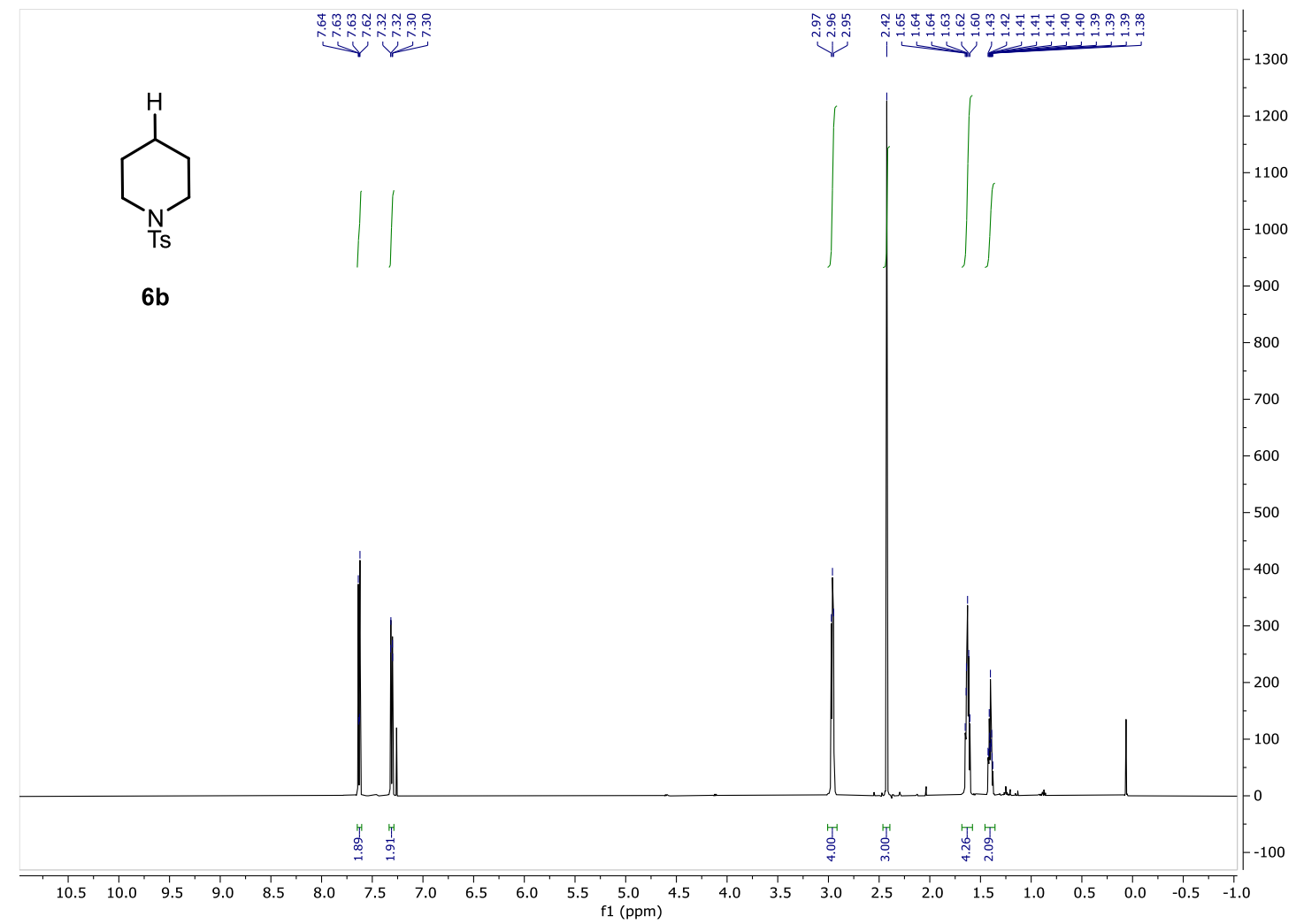

${ }^{13} \mathrm{C}$ NMR (126 MHz, $\left.\mathrm{CDCl}_{3}\right)$

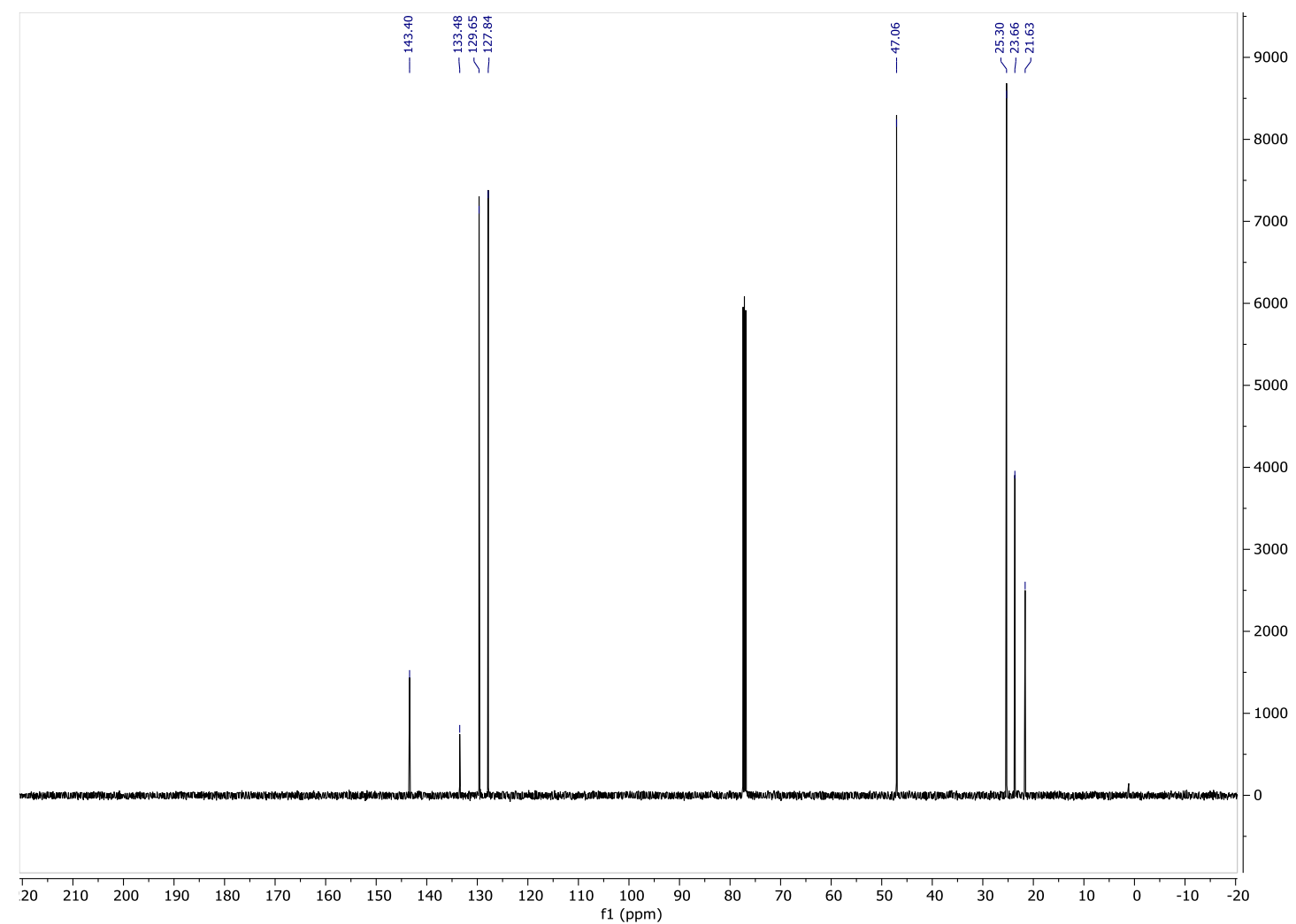


${ }^{1} \mathrm{H}$ NMR (400 MHz, $\mathrm{CDCl}_{3}$ )

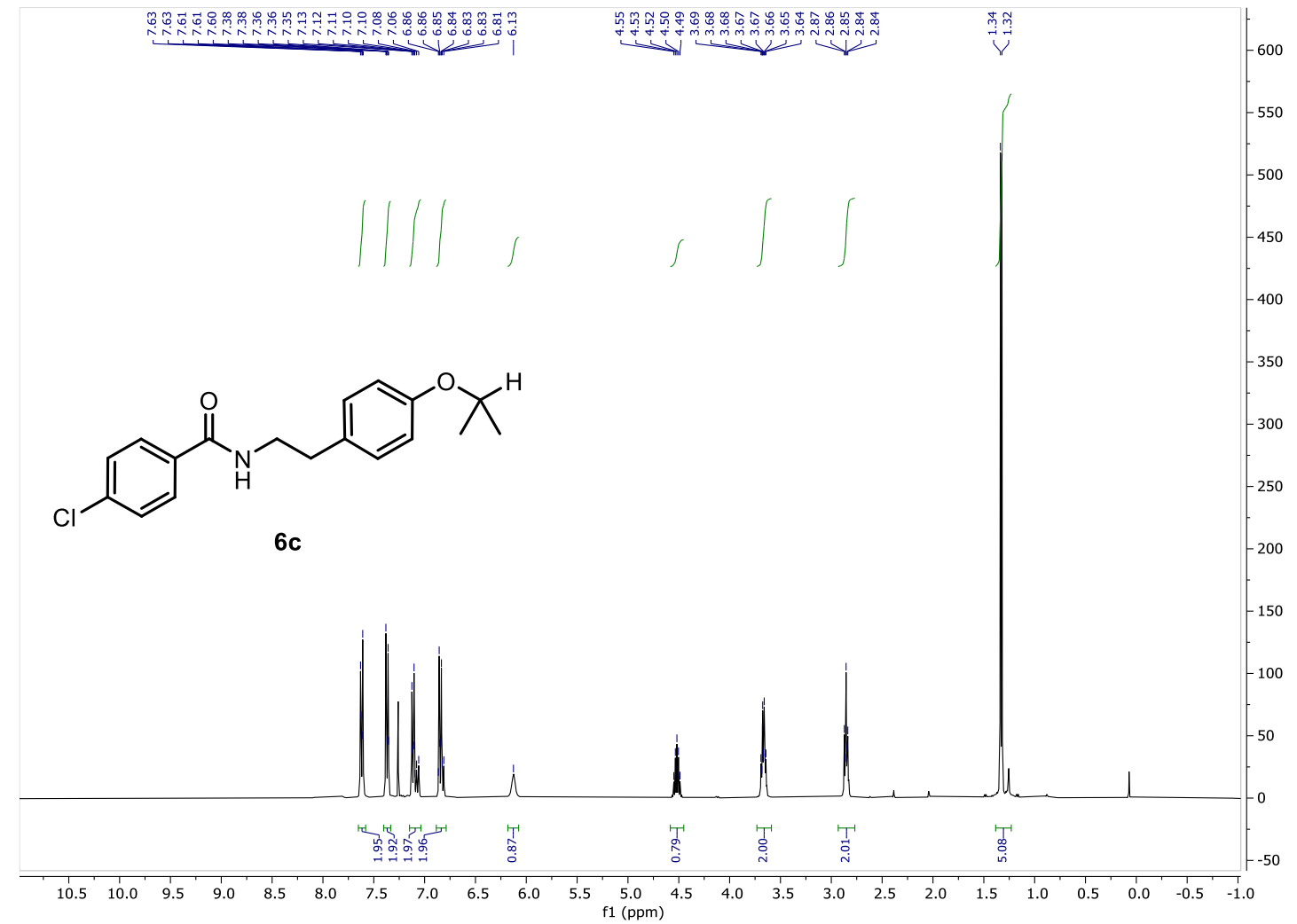

${ }^{13} \mathrm{C}$ NMR (101 MHz, $\mathrm{CDCl}_{3}$ )

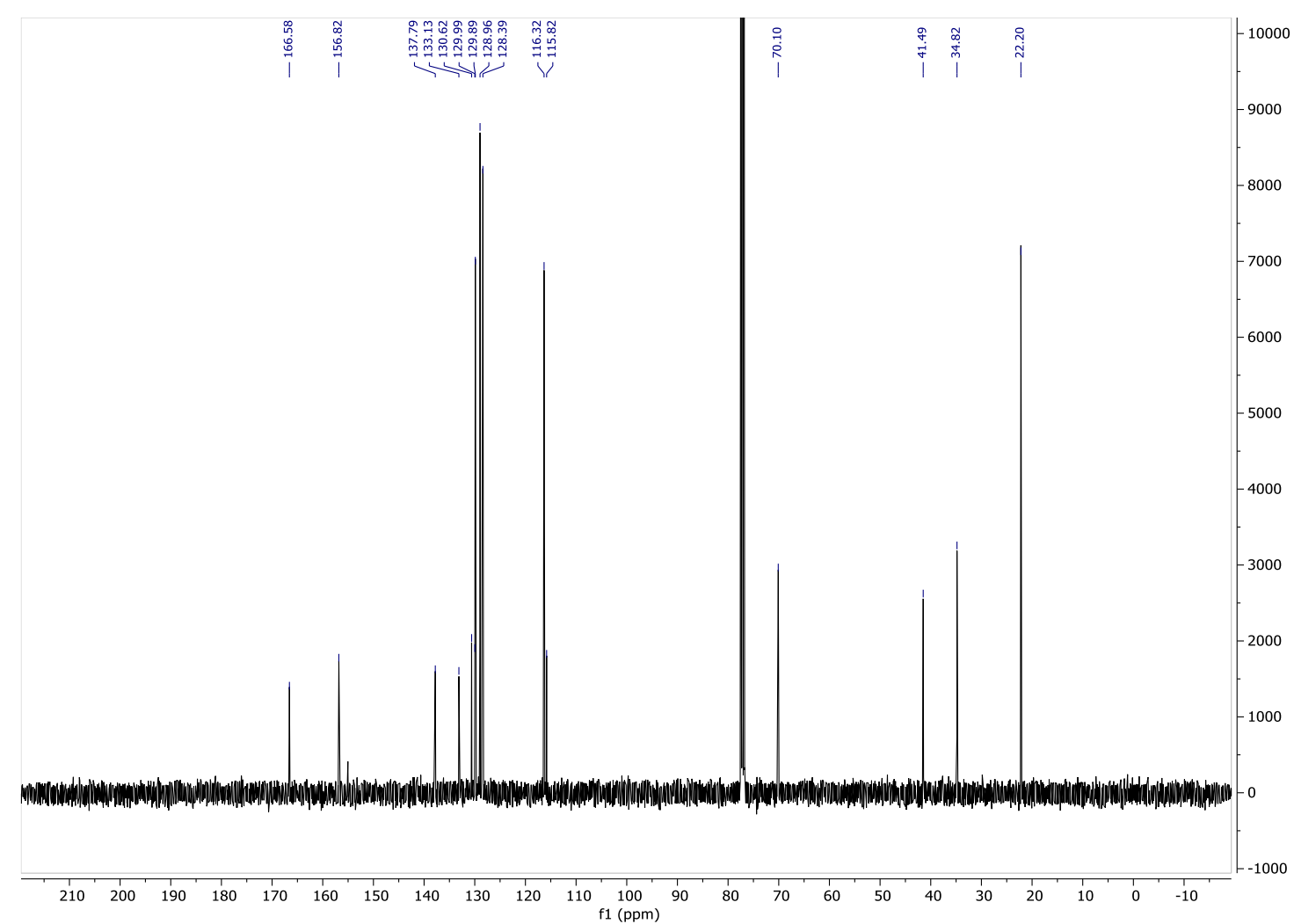


${ }^{1} \mathrm{H}$ NMR (400 MHz, $\mathrm{CDCl}_{3}$ )

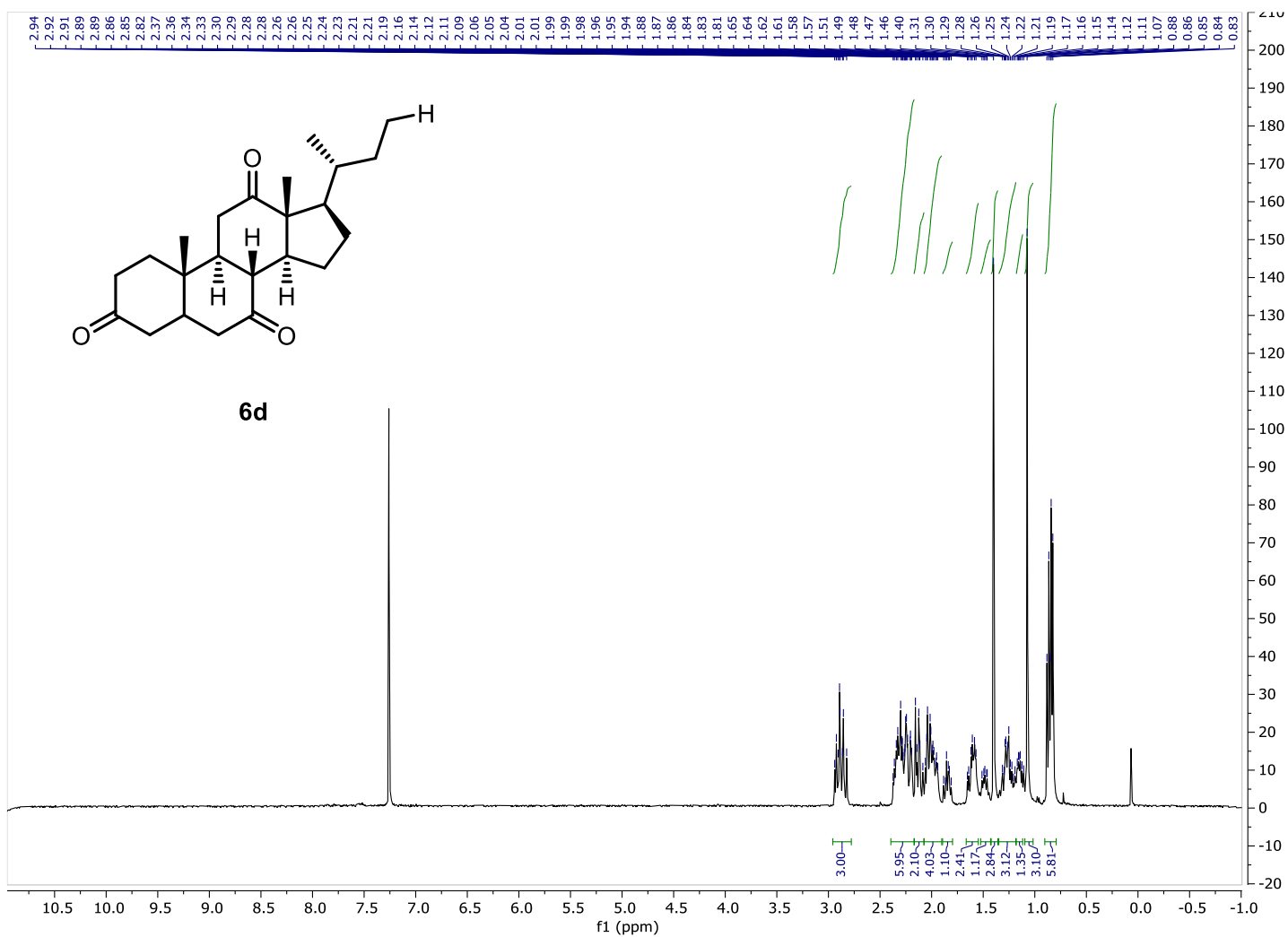

${ }^{13} \mathrm{C}$ NMR $\left(101 \mathrm{MHz}, \mathrm{CDCl}_{3}\right)$

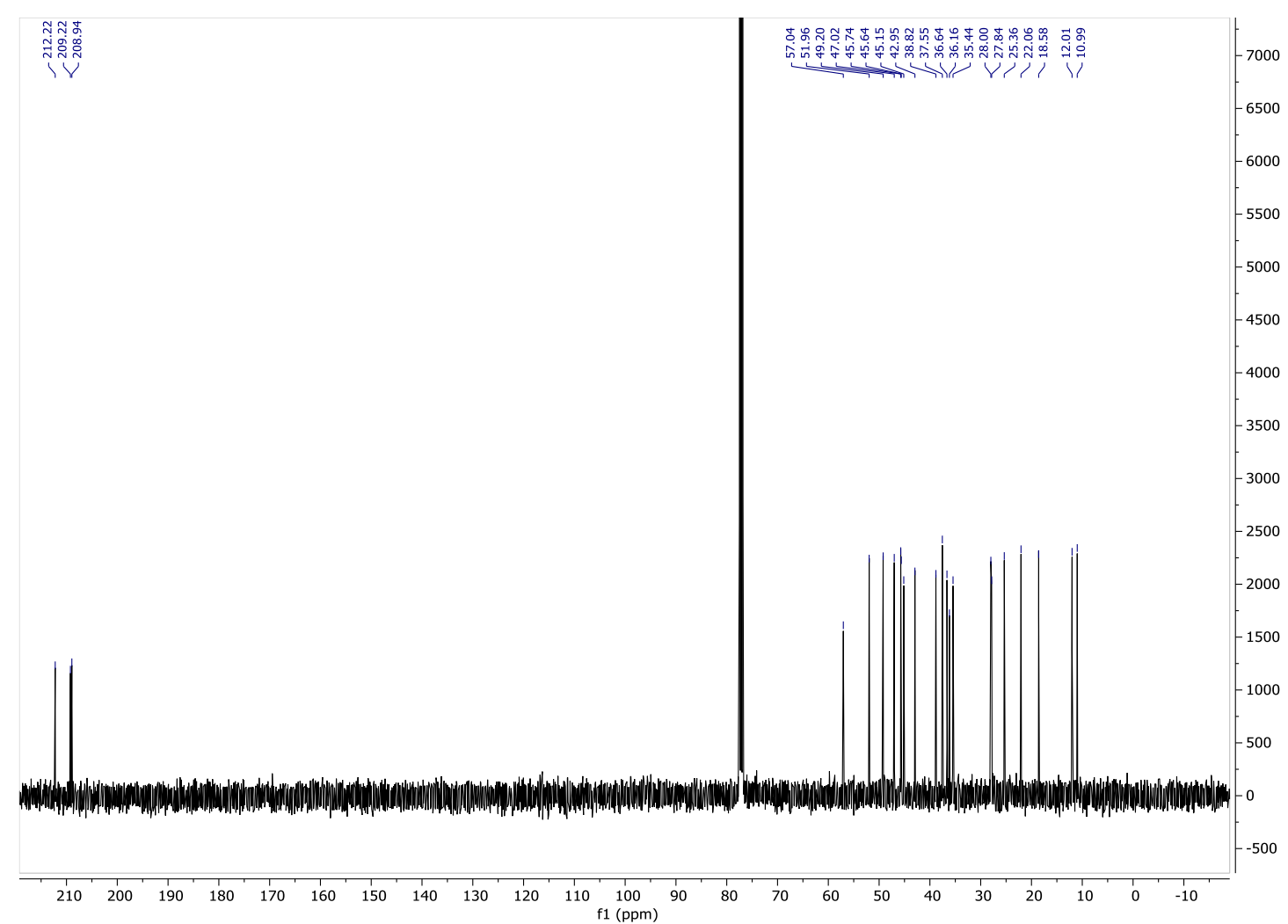


${ }^{1} \mathrm{H}$ NMR (400 MHz, $\mathrm{CDCl}_{3}$ )

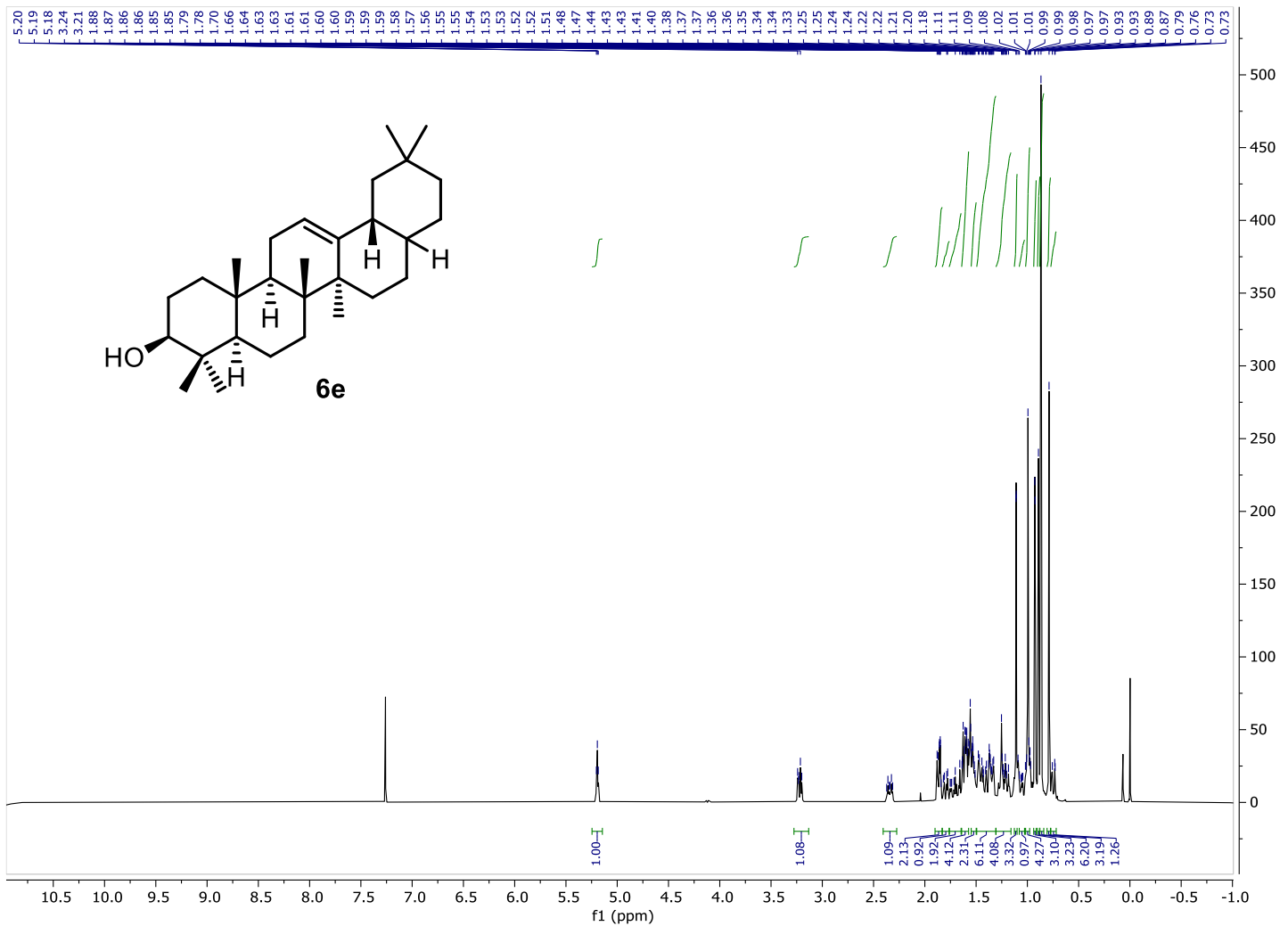

${ }^{13} \mathrm{C}$ NMR $\left(101 \mathrm{MHz}, \mathrm{CDCl}_{3}\right)$

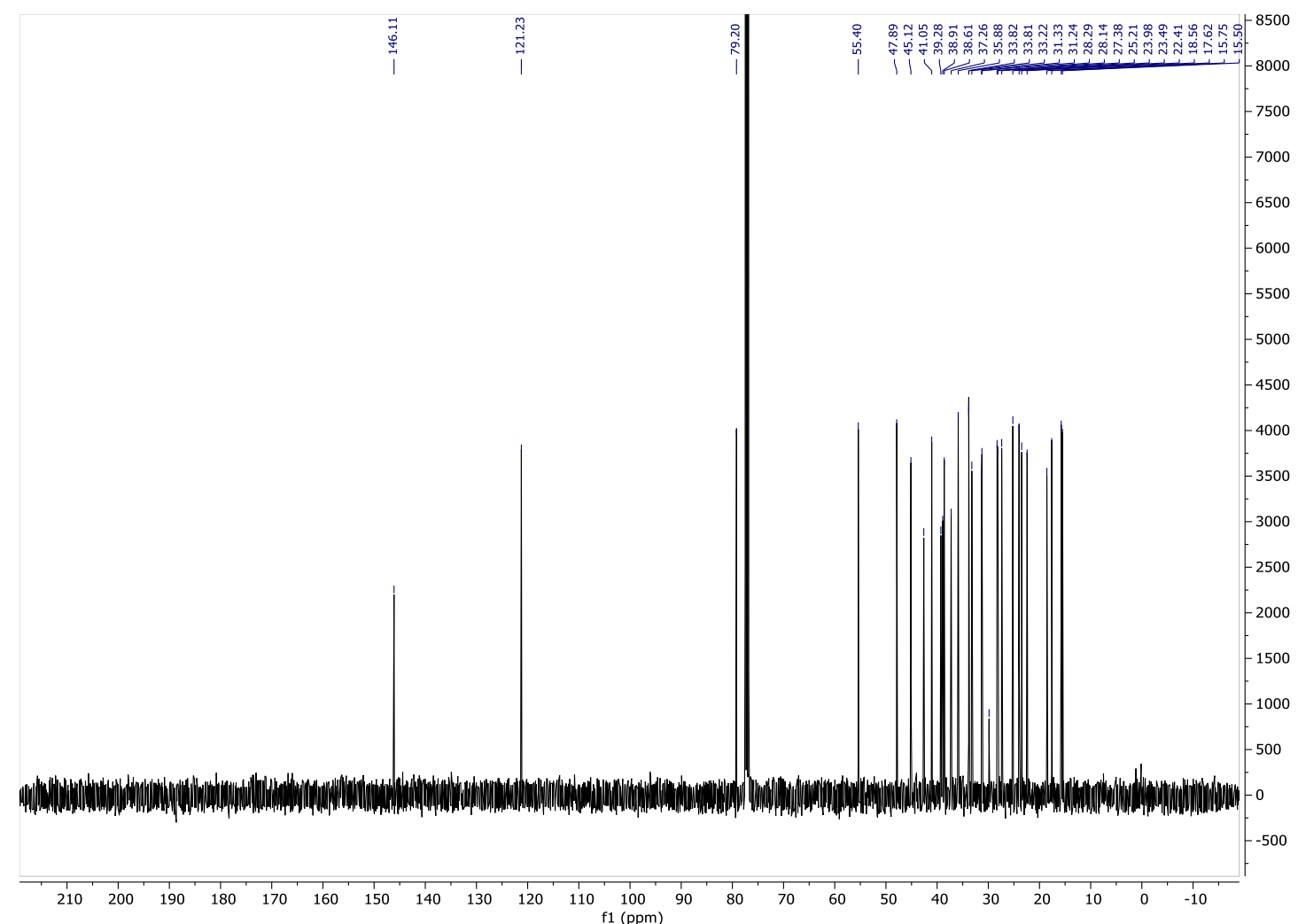


${ }^{1} \mathrm{H}$ NMR (400 MHz, Acetone- $d_{6}$ )

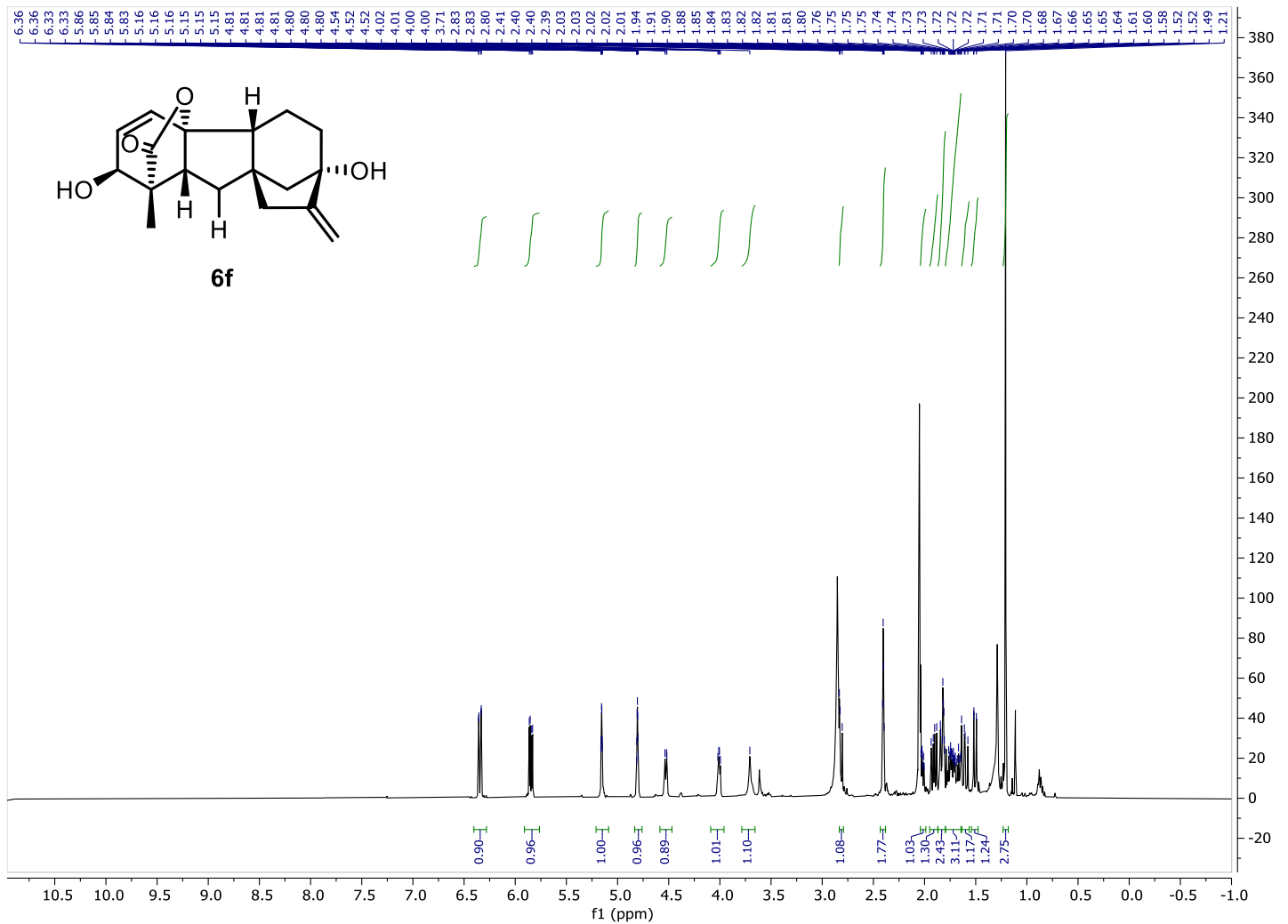

${ }^{13} \mathrm{C}$ NMR (101 MHz, Acetone- $d_{6}$ )

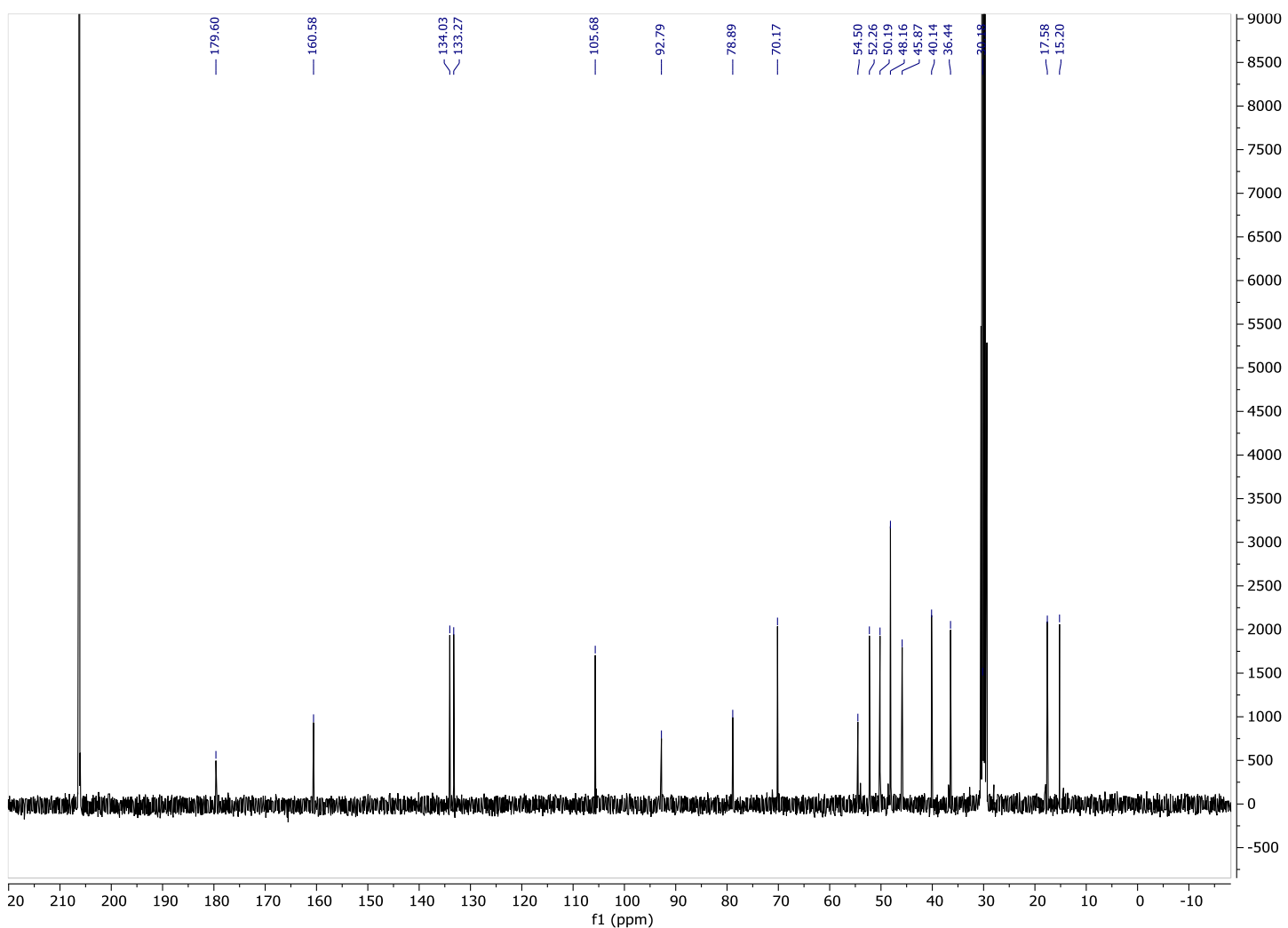


${ }^{1} \mathrm{H}$ NMR (400 MHz, $\mathrm{CDCl}_{3}$ )

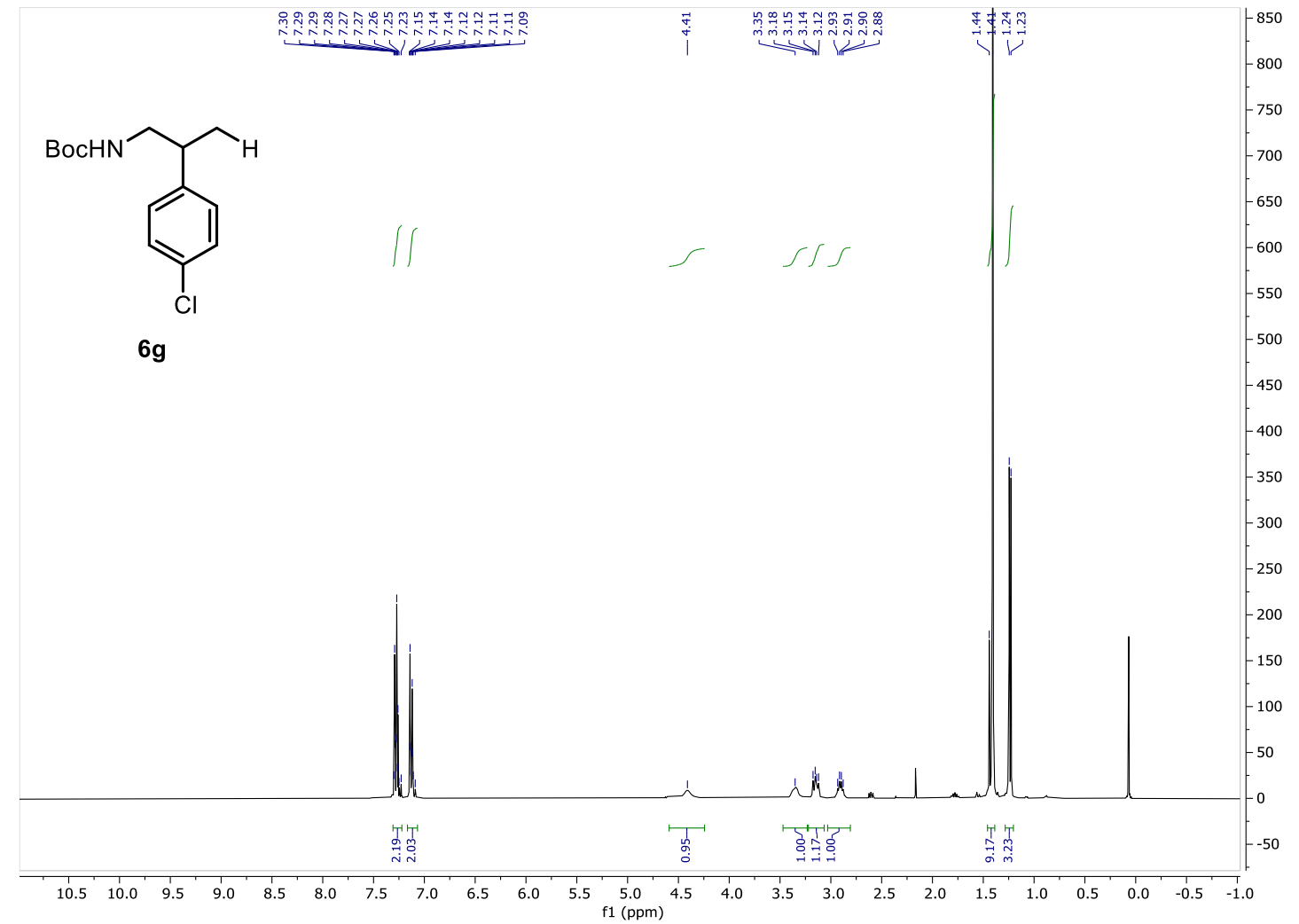

${ }^{13} \mathrm{C}$ NMR $\left(101 \mathrm{MHz}, \mathrm{CDCl}_{3}\right)$

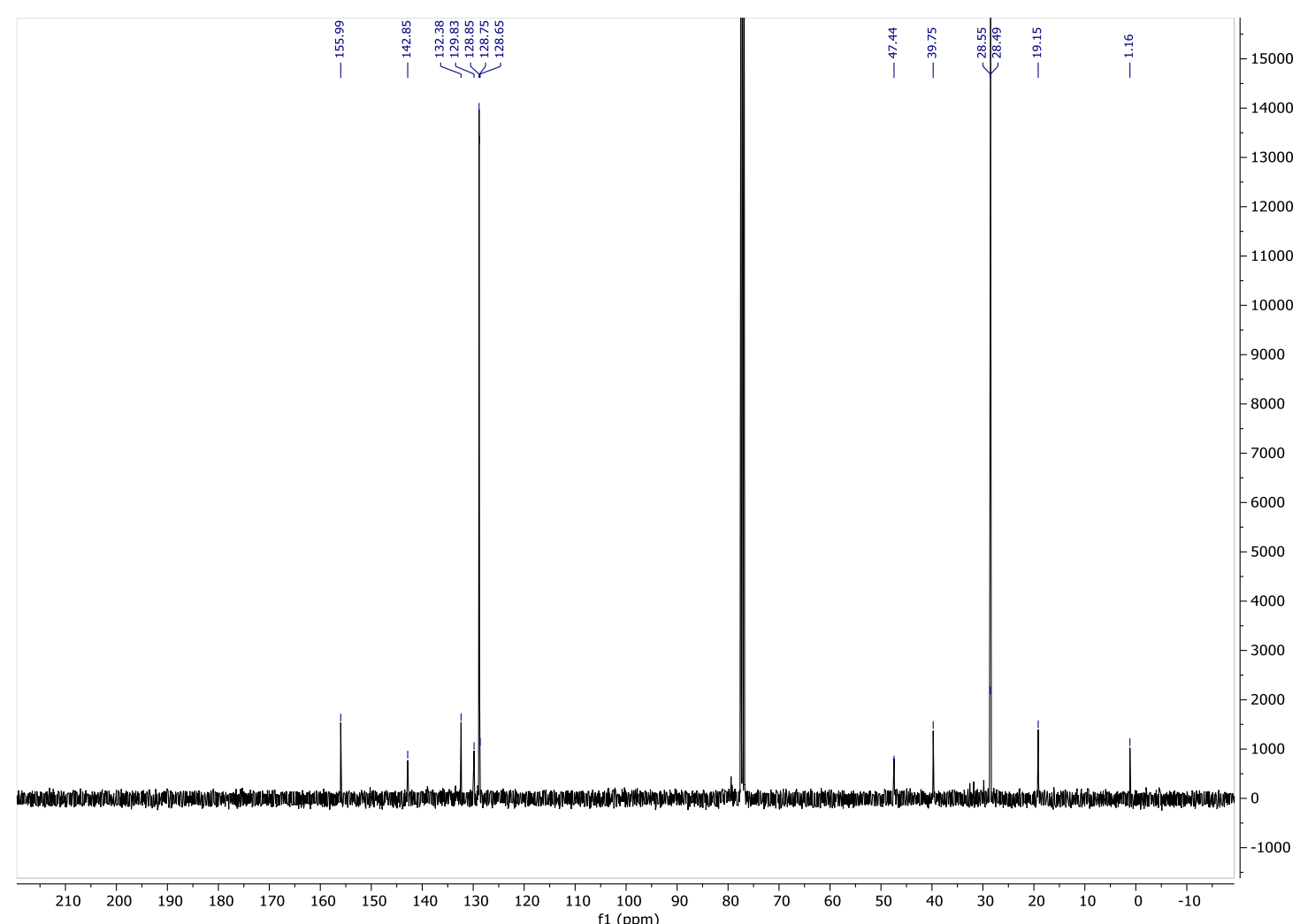


${ }^{1} \mathrm{H}$ NMR (400 MHz, $\mathrm{CDCl}_{3}$ )

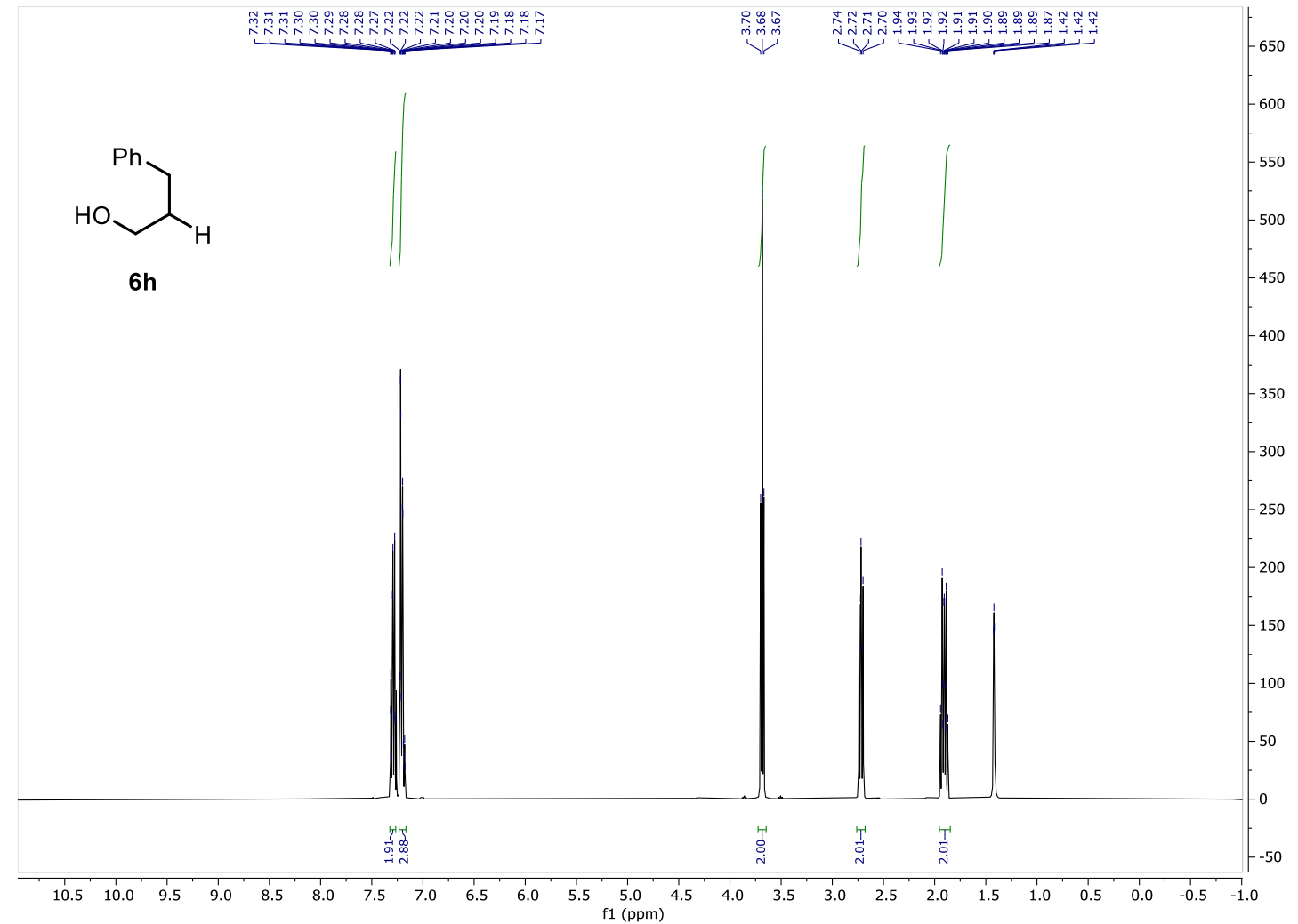

${ }^{13} \mathrm{C}$ NMR $\left(101 \mathrm{MHz}, \mathrm{CDCl}_{3}\right)$

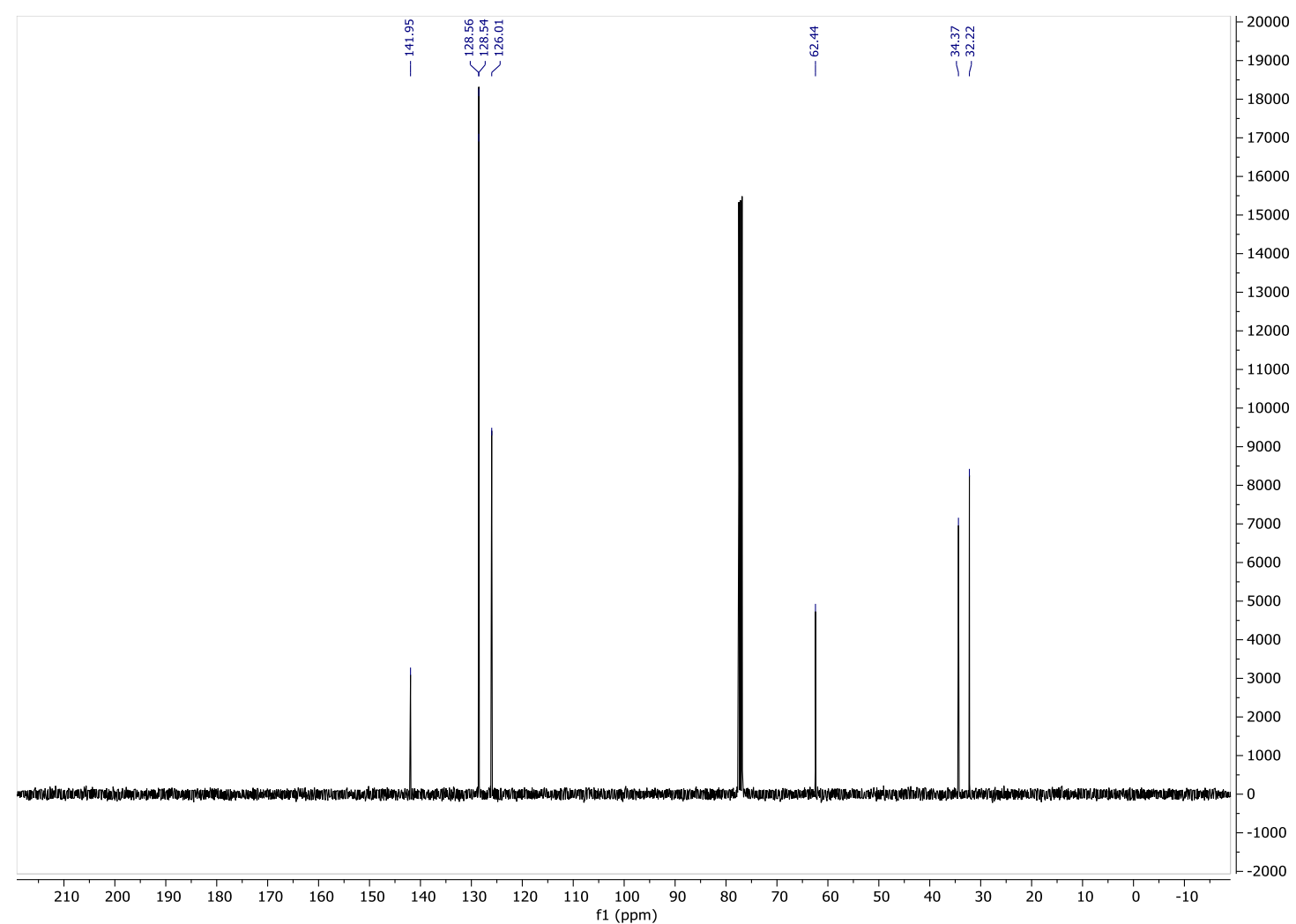


${ }^{1} \mathrm{H}$ NMR (400 MHz, $\mathrm{CDCl}_{3}$ )

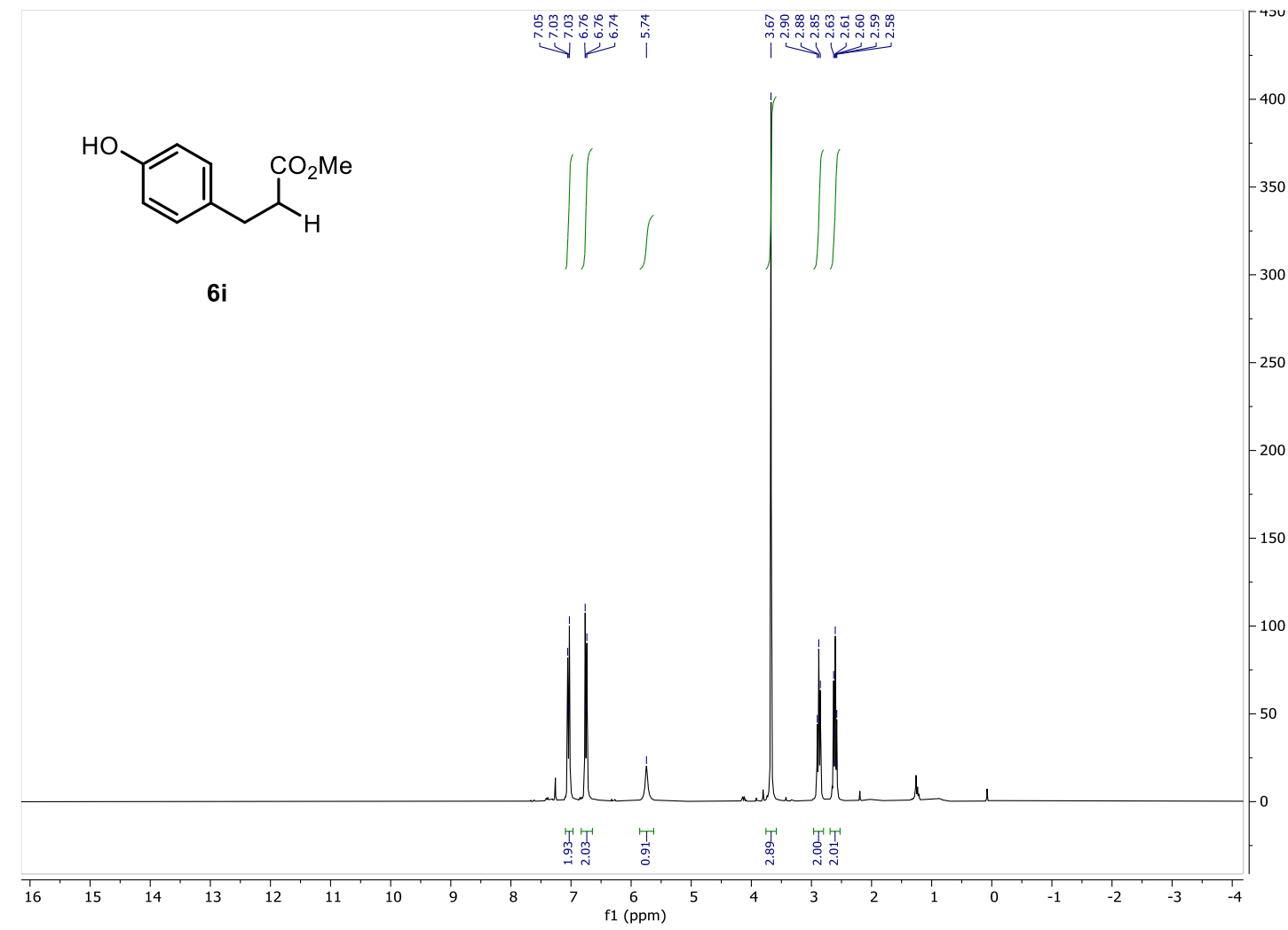

${ }^{13} \mathrm{C}$ NMR $\left(101 \mathrm{MHz}, \mathrm{CDCl}_{3}\right)$

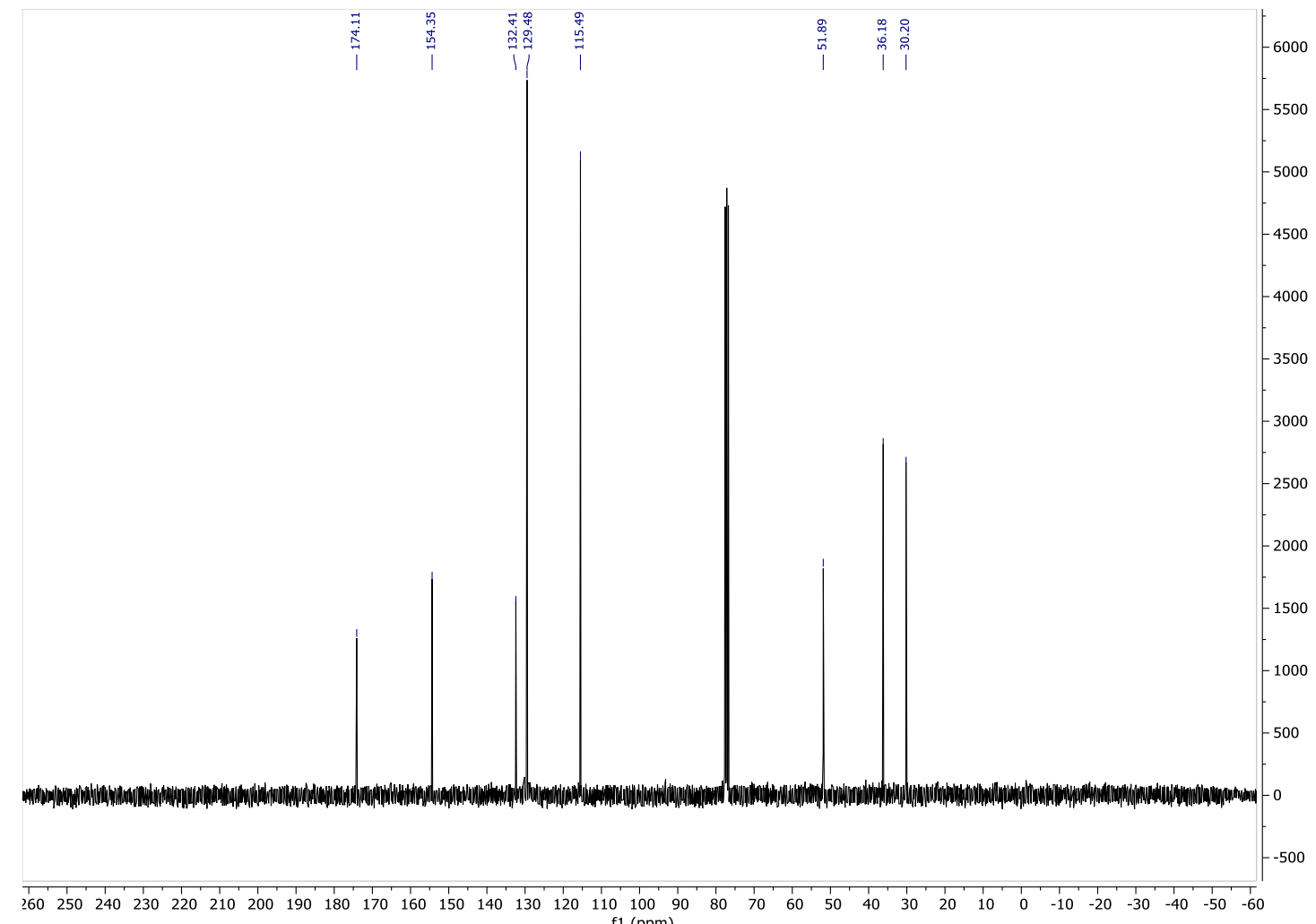


${ }^{1} \mathrm{H}$ NMR (300 MHz, $\left.\mathrm{CDCl}_{3}\right)$

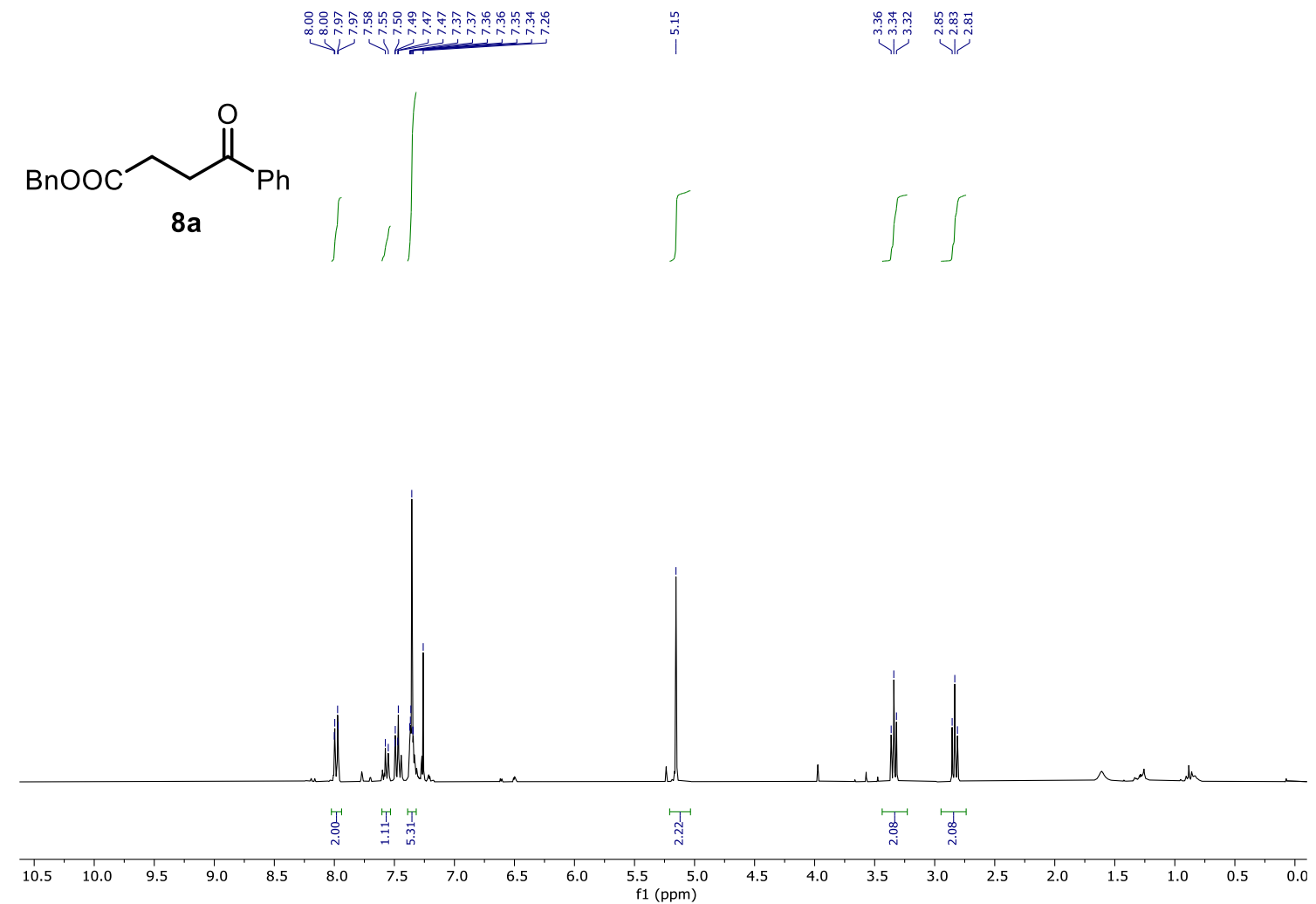

${ }^{13} \mathrm{C}$ NMR $\left(75 \mathrm{MHz}, \mathrm{CDCl}_{3}\right)$

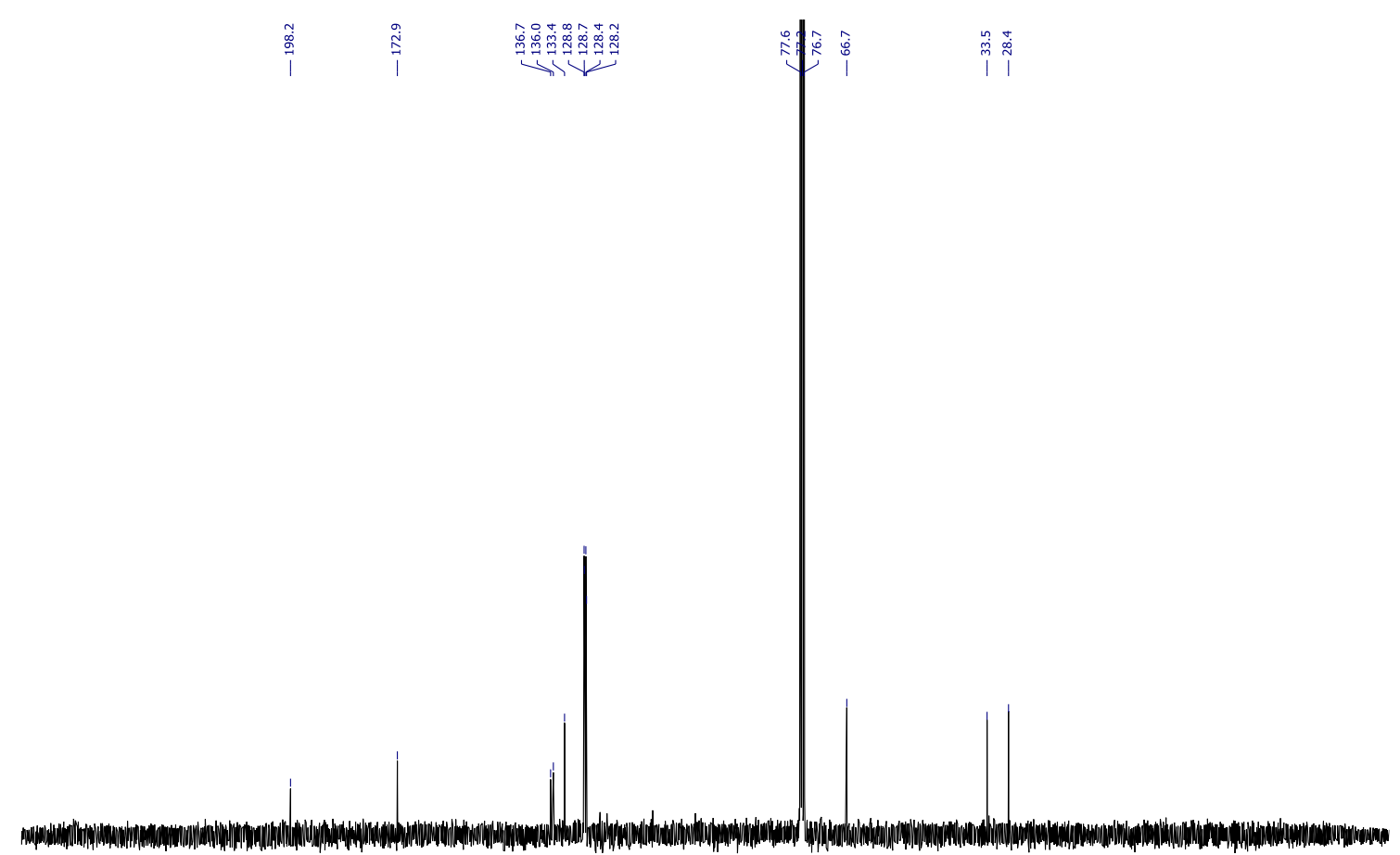

$\begin{array}{llllllllllllllllllllllllllllllllllllllllllllllll}260 & 250 & 240 & 230 & 220 & 210 & 200 & 190 & 180 & 170 & 160 & 150 & 140 & 130 & 120 & 110 & 100 & 90 & 80 & 70 & 60 & 50 & 40 & 30 & 20 & 10 & 0 & -10 & -20 & -30 & -40 & -50 & -6\end{array}$ 
${ }^{1} \mathrm{H}$ NMR (300 MHz, $\mathrm{CDCl}_{3}$ )

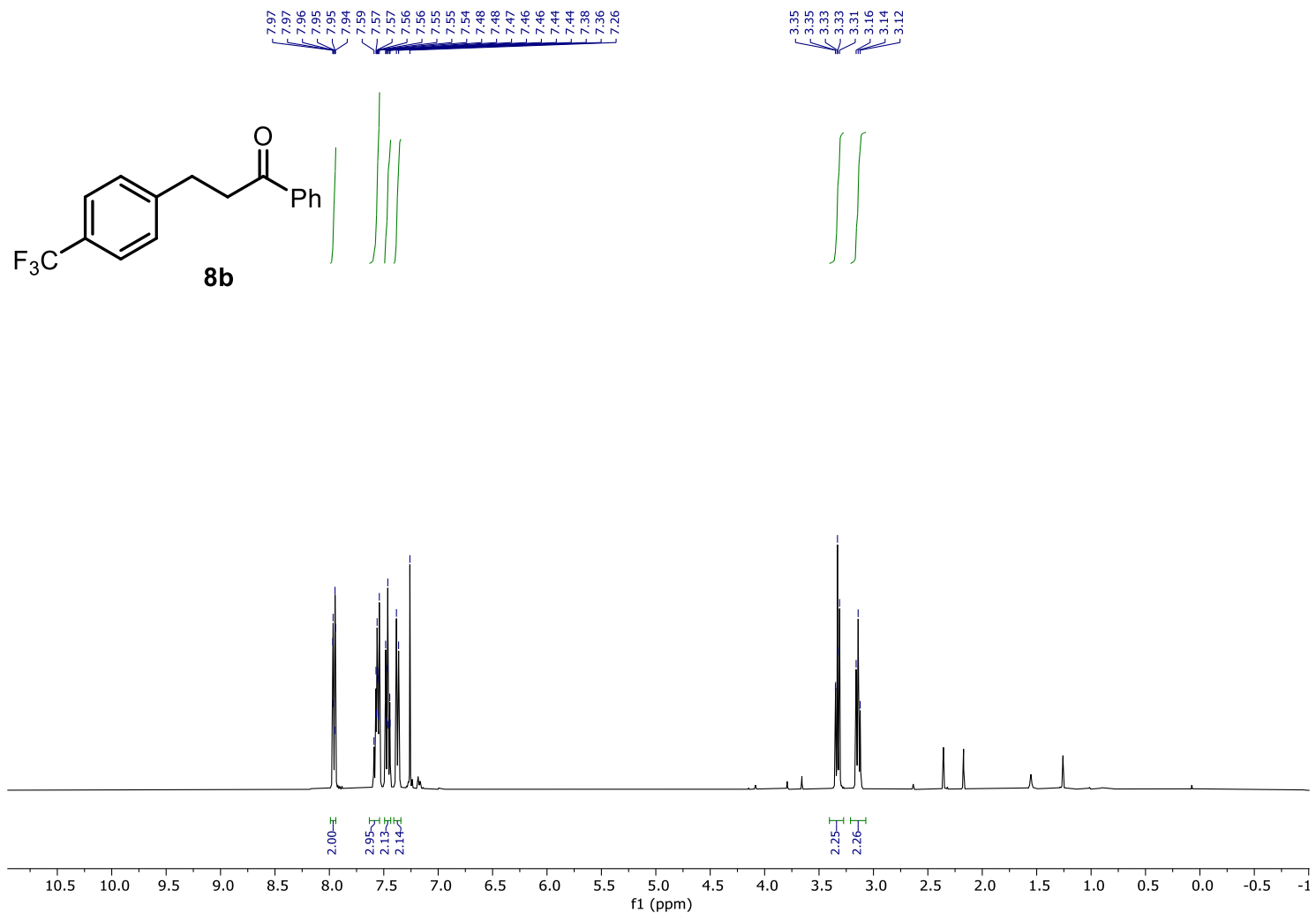

${ }^{13} \mathrm{C}$ NMR $\left(75 \mathrm{MHz}, \mathrm{CDCl}_{3}\right)$

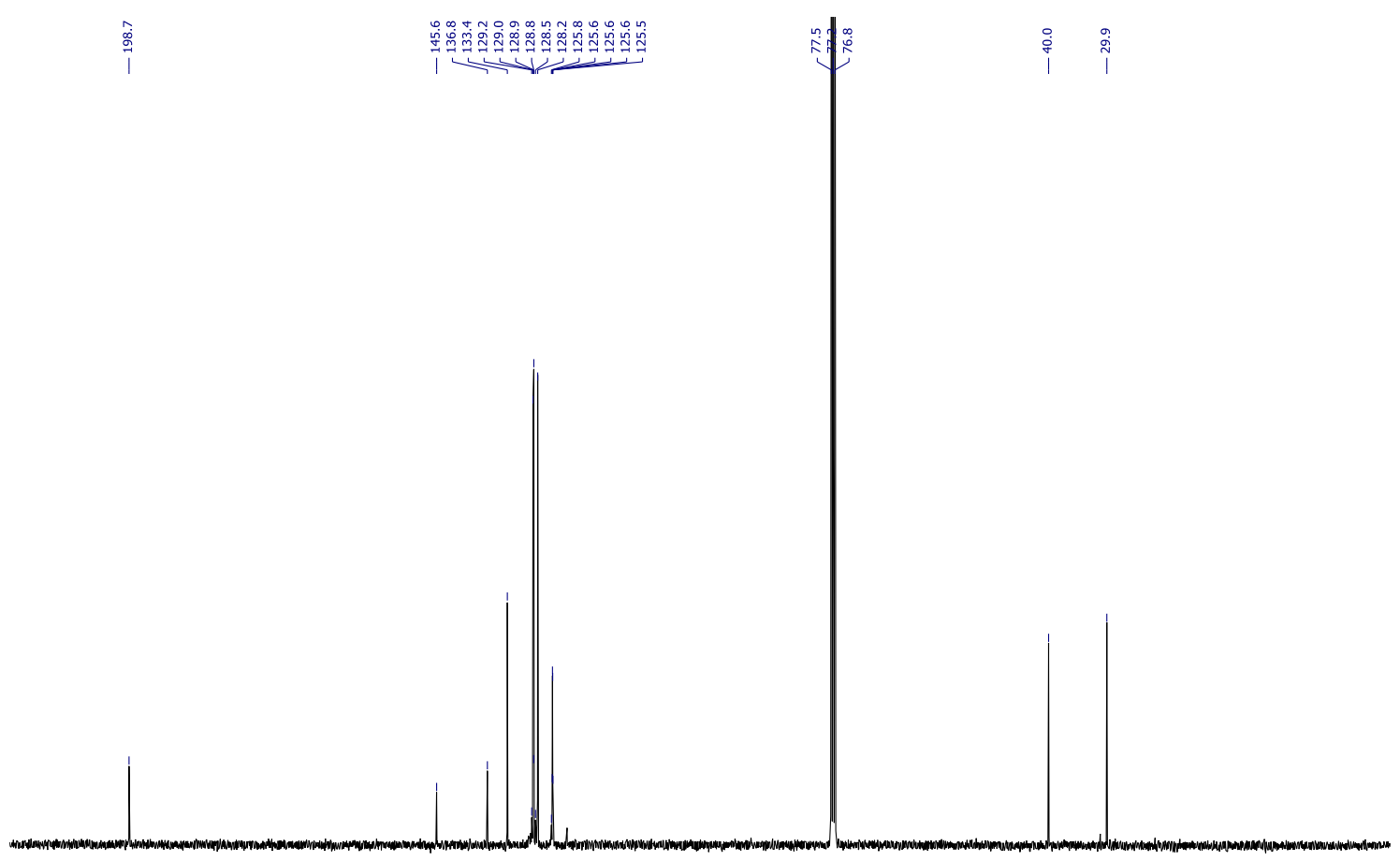

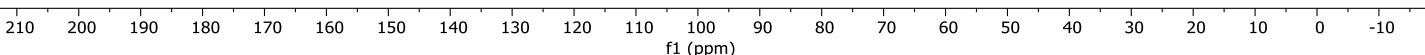


${ }^{19} \mathrm{~F}$ NMR $\left(376 \mathrm{MHz}, \mathrm{CDCl}_{3}\right.$ )

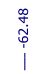

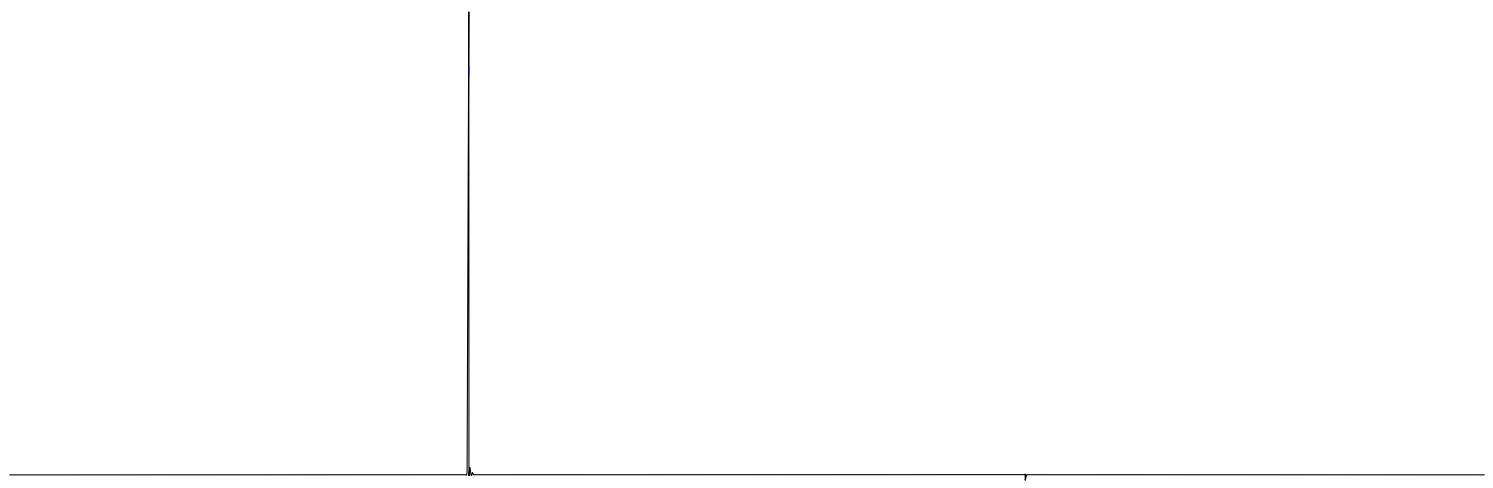

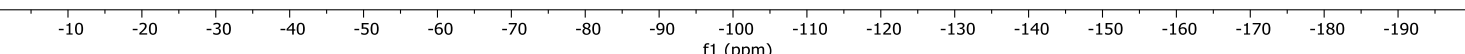


${ }^{1} \mathrm{H}$ NMR (400 MHz, $\left.\mathrm{CDCl}_{3}\right)$

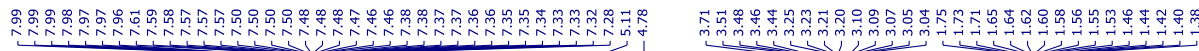<smiles>COC(=O)C(CC(=O)OCc1ccccc1)C(=O)c1ccccc1</smiles>
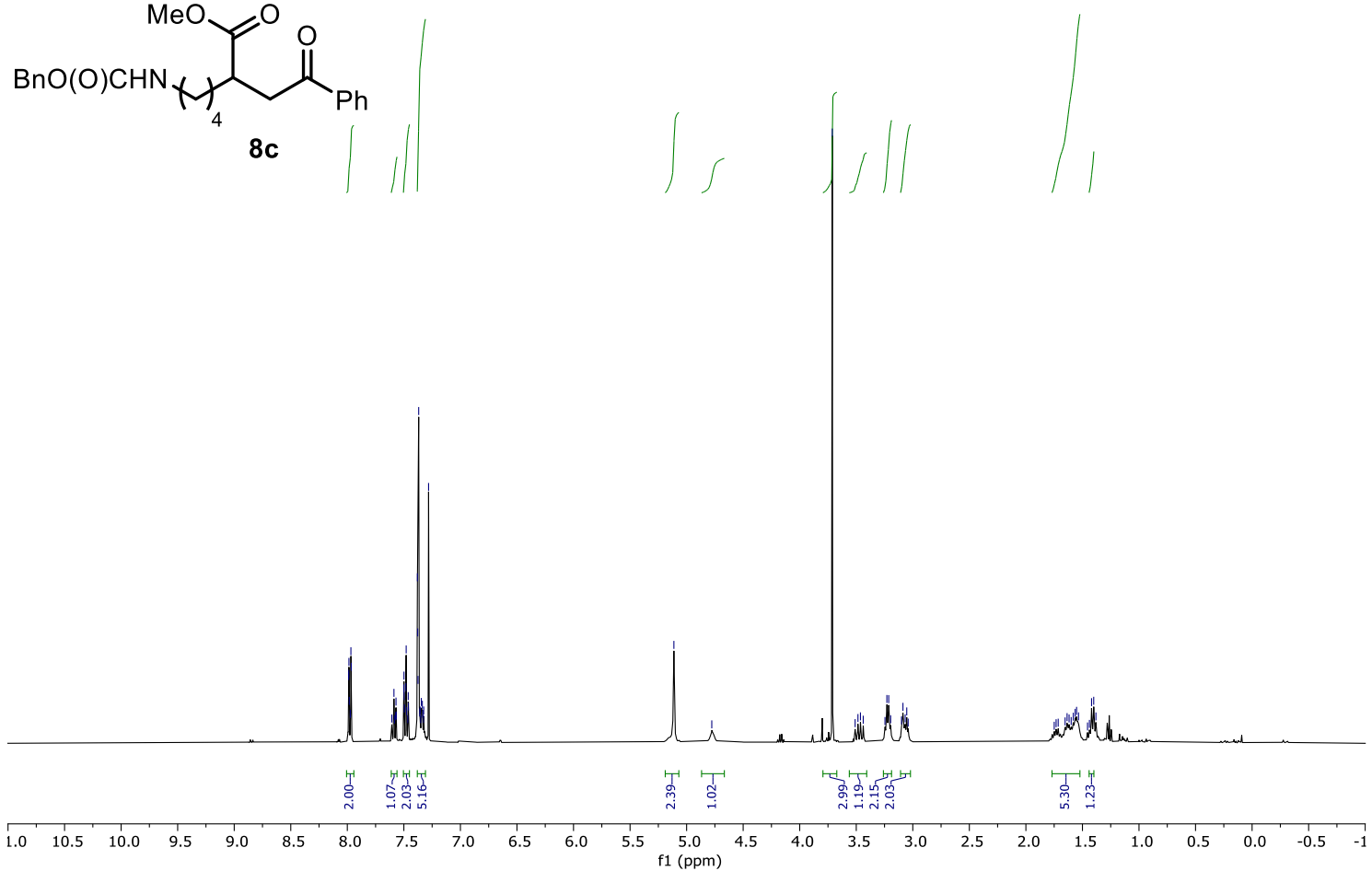

${ }^{13} \mathrm{C}$ NMR $\left(101 \mathrm{MHz}, \mathrm{CDCl}_{3}\right)$

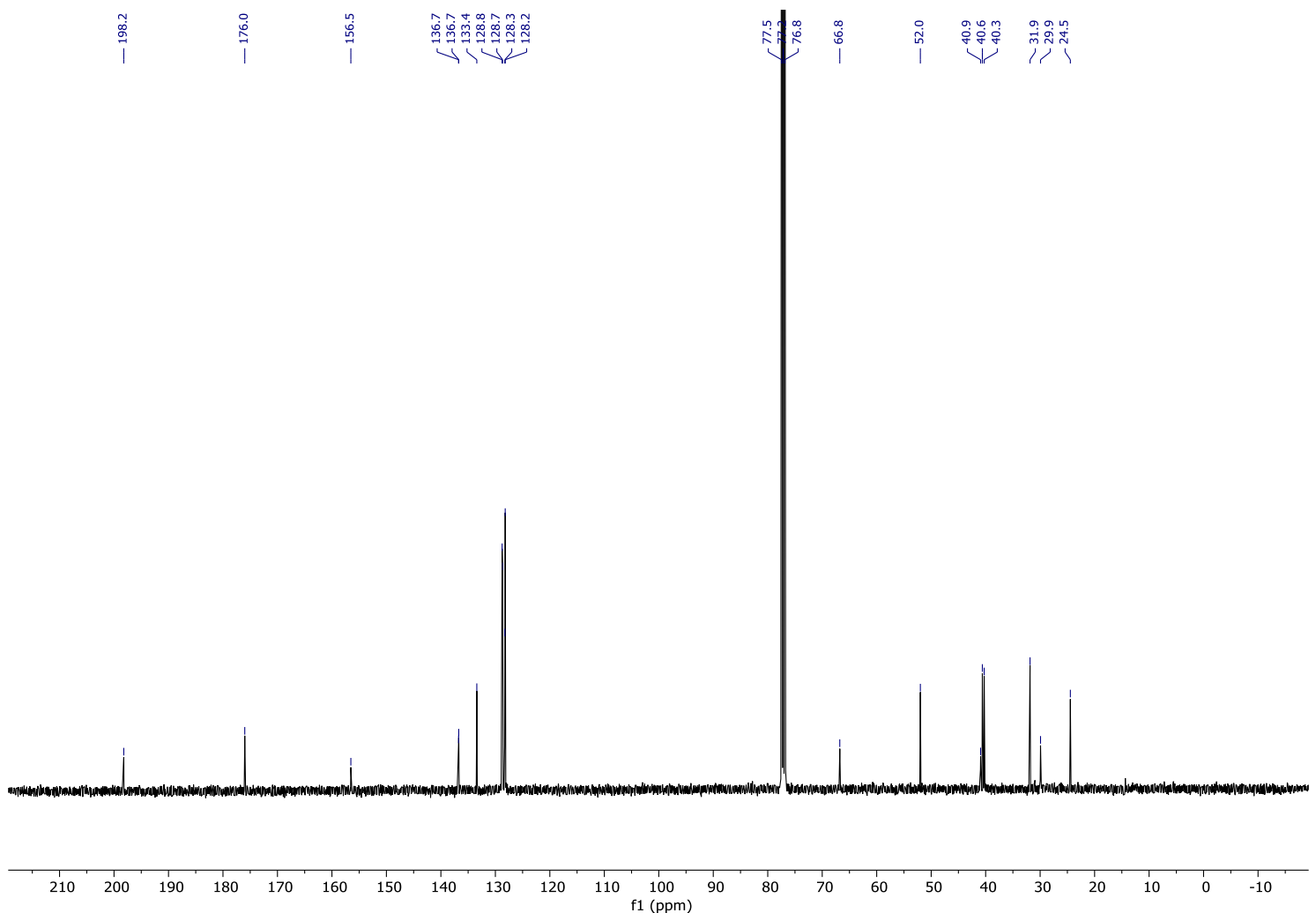


${ }^{1} \mathrm{H}$ NMR (300 MHz, $\left.\mathrm{CDCl}_{3}\right)$

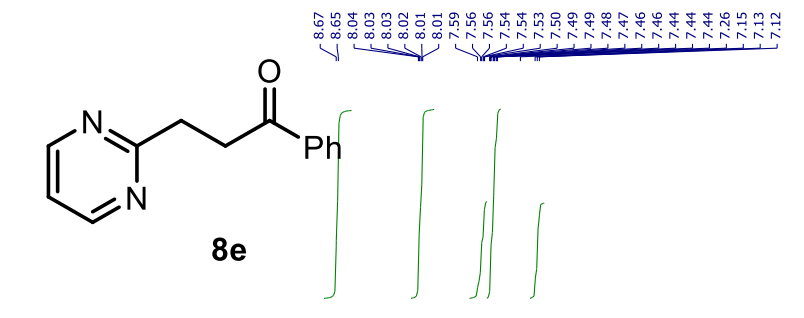

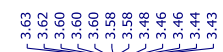
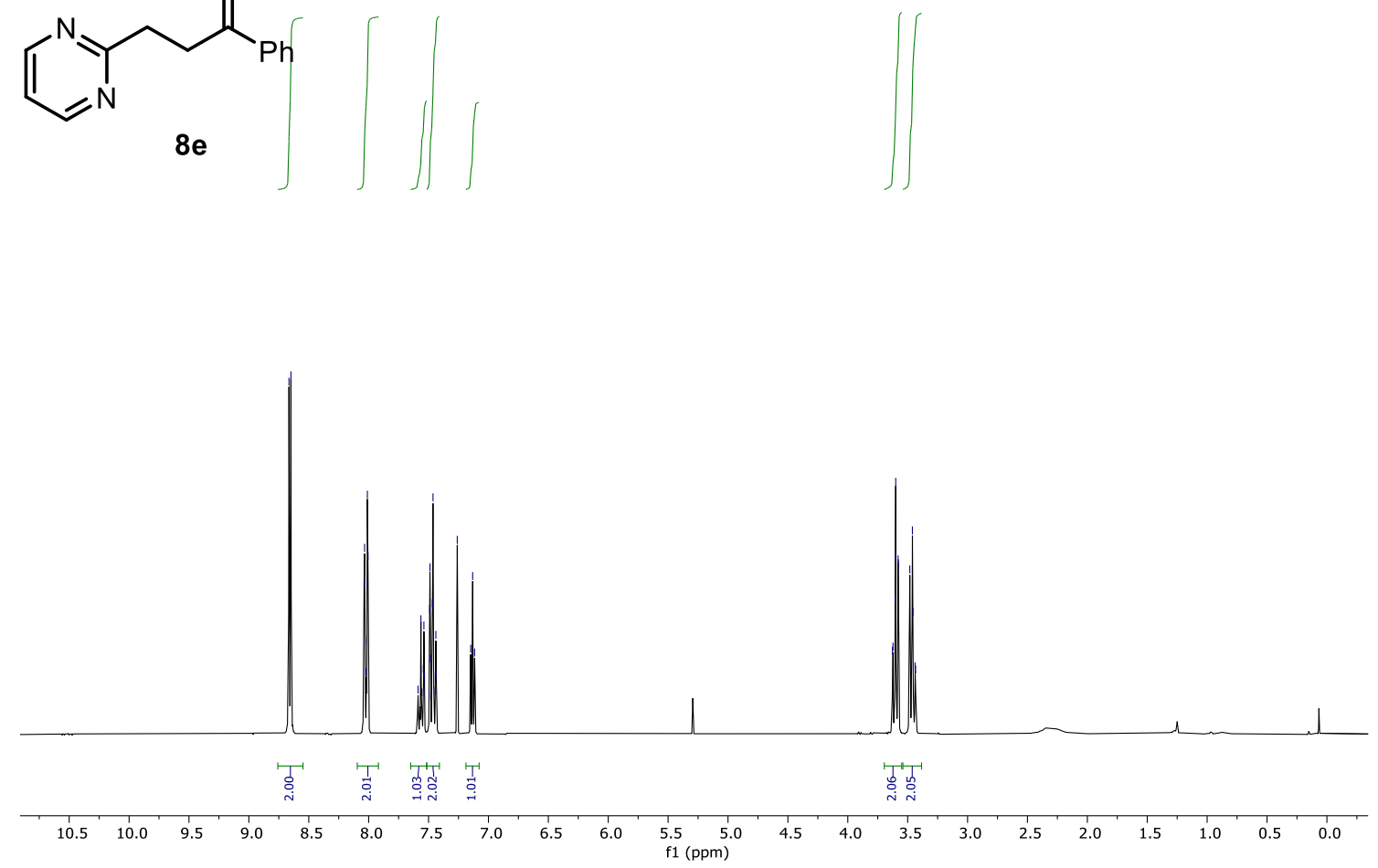

${ }^{13} \mathrm{C}$ NMR $\left(75 \mathrm{MHz}, \mathrm{CDCl}_{3}\right)$

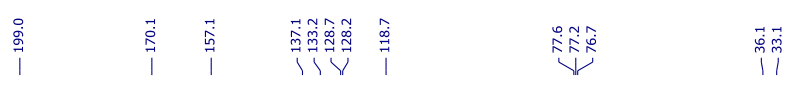

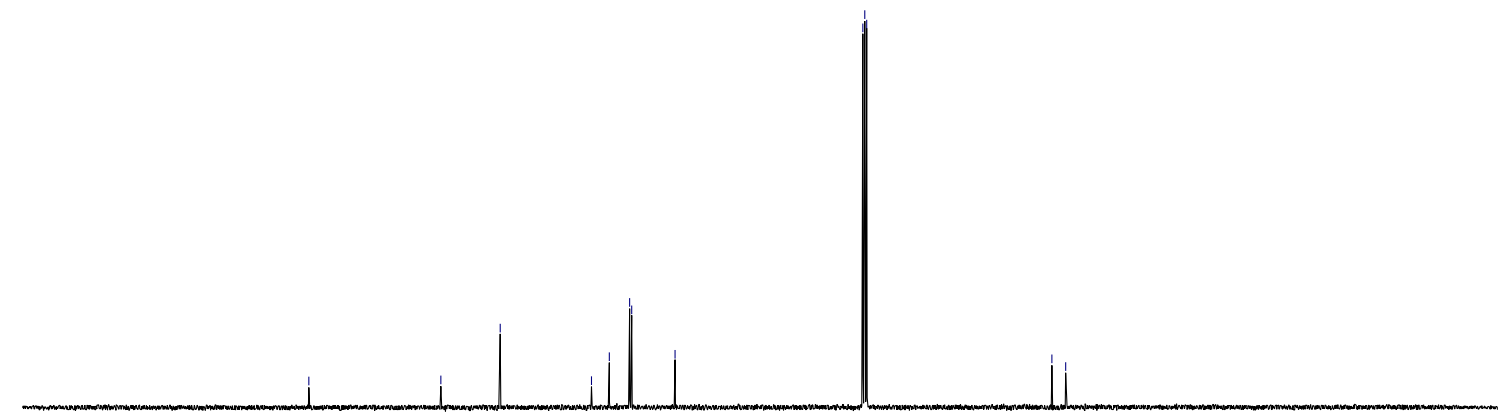

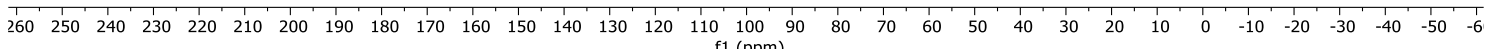


${ }^{1} \mathrm{H}$ NMR (400 MHz, $\mathrm{CDCl}_{3}$ )

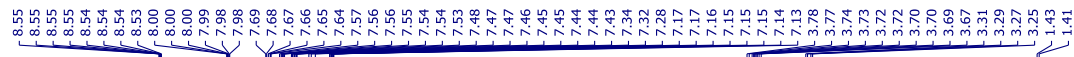<smiles>C#CCC(=O)c1ccccc1</smiles>
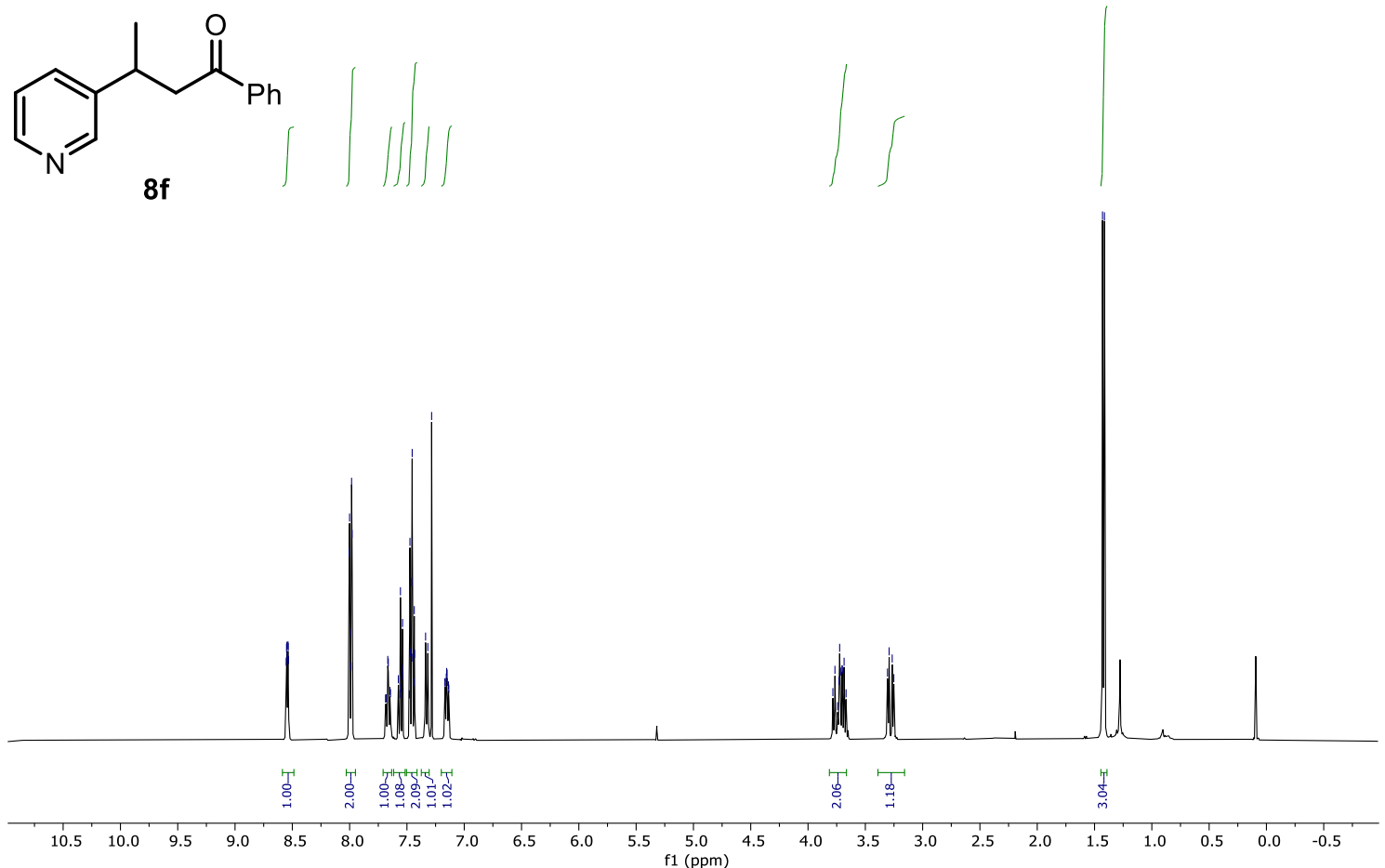

${ }^{13} \mathrm{C}$ NMR $\left(101 \mathrm{MHz}, \mathrm{CDCl}_{3}\right)$

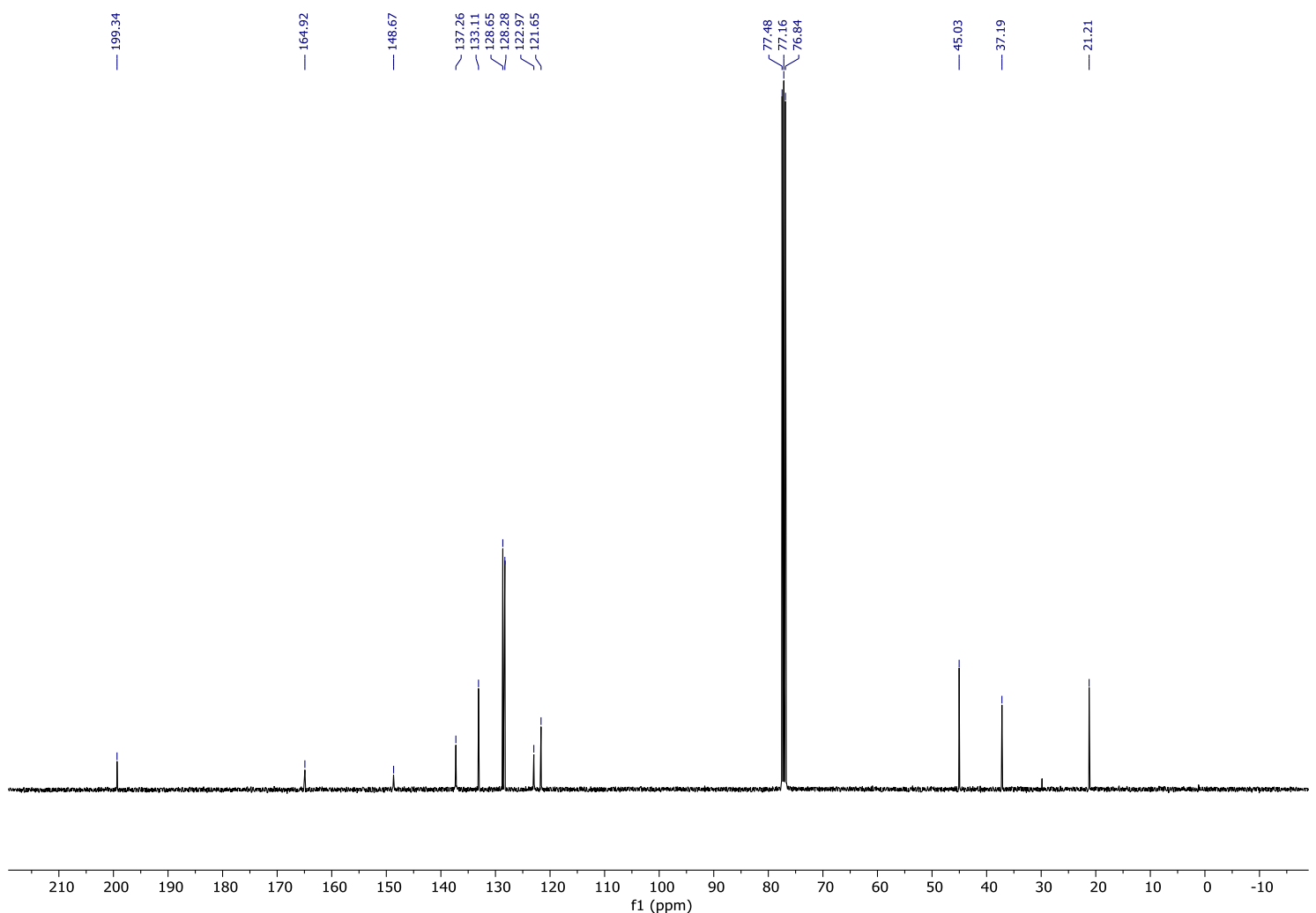


${ }^{1} \mathrm{H}$ NMR (400 MHz, $\left.\mathrm{CDCl}_{3}\right)$

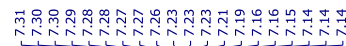

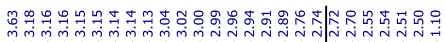<smiles>CC(=O)C(CC(=O)C(C)(C)C)Cc1ccccc1</smiles>

$8 \mathrm{~g}$<smiles>C1=CC=C1</smiles>

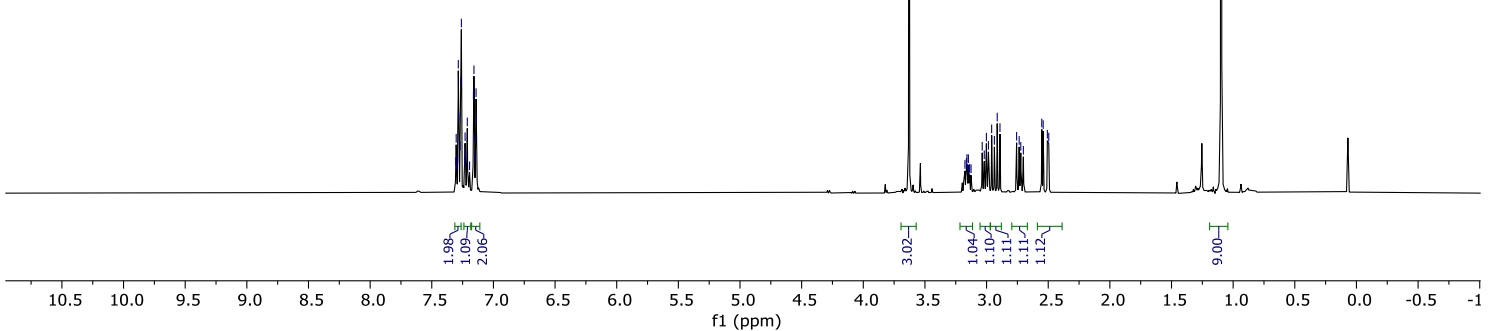

${ }^{13} \mathrm{C}$ NMR (101 MHz, $\left.\mathrm{CDCl}_{3}\right)$

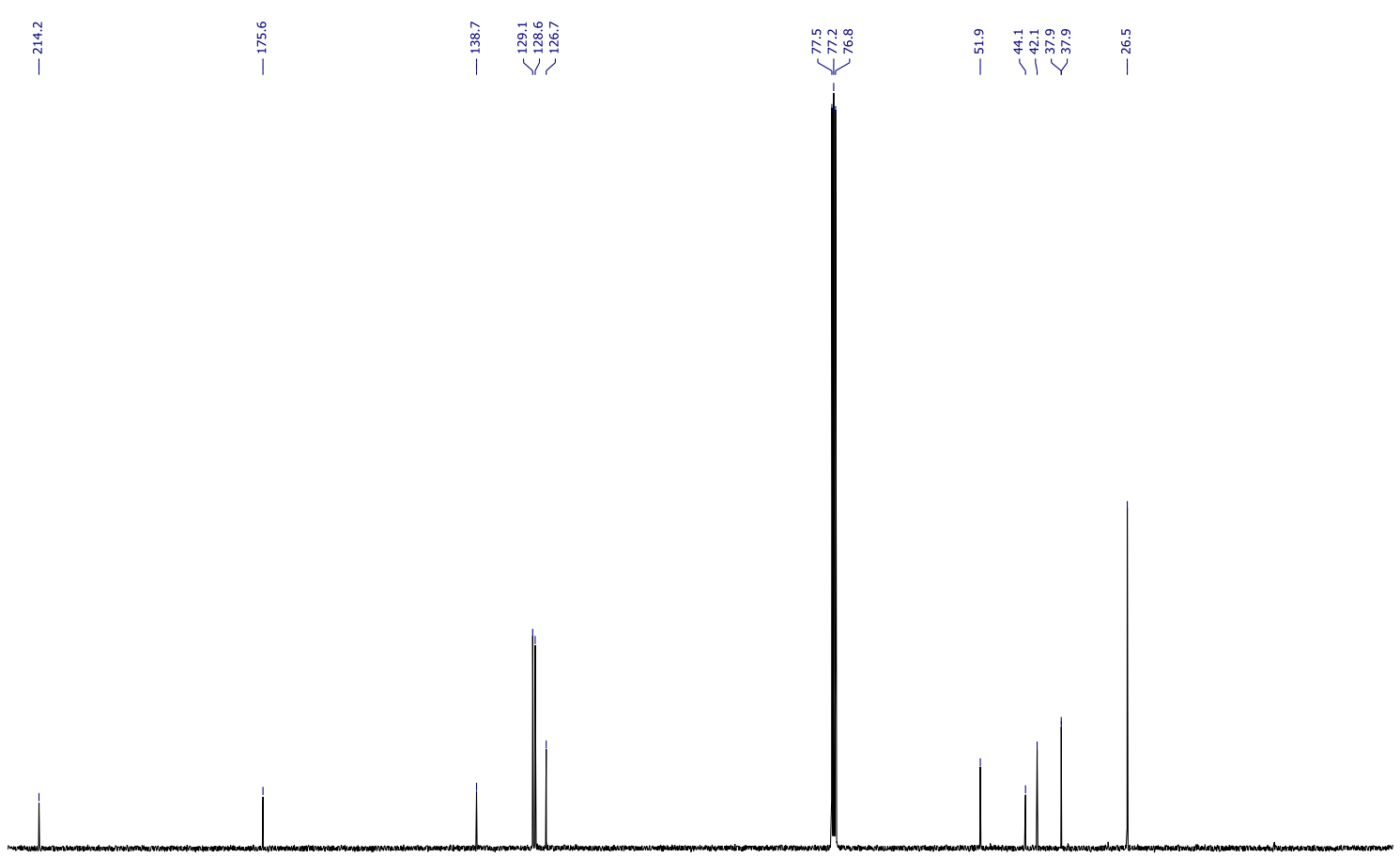

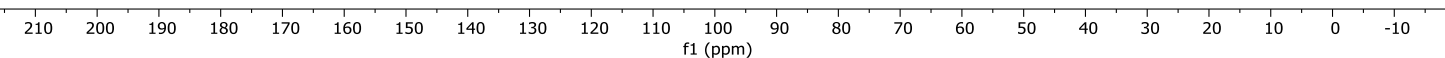


${ }^{1} \mathrm{H}$ NMR (400 MHz, $\left.\mathrm{CDCl}_{3}\right)$

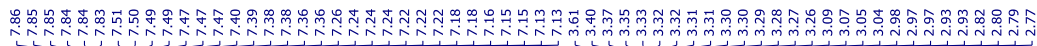
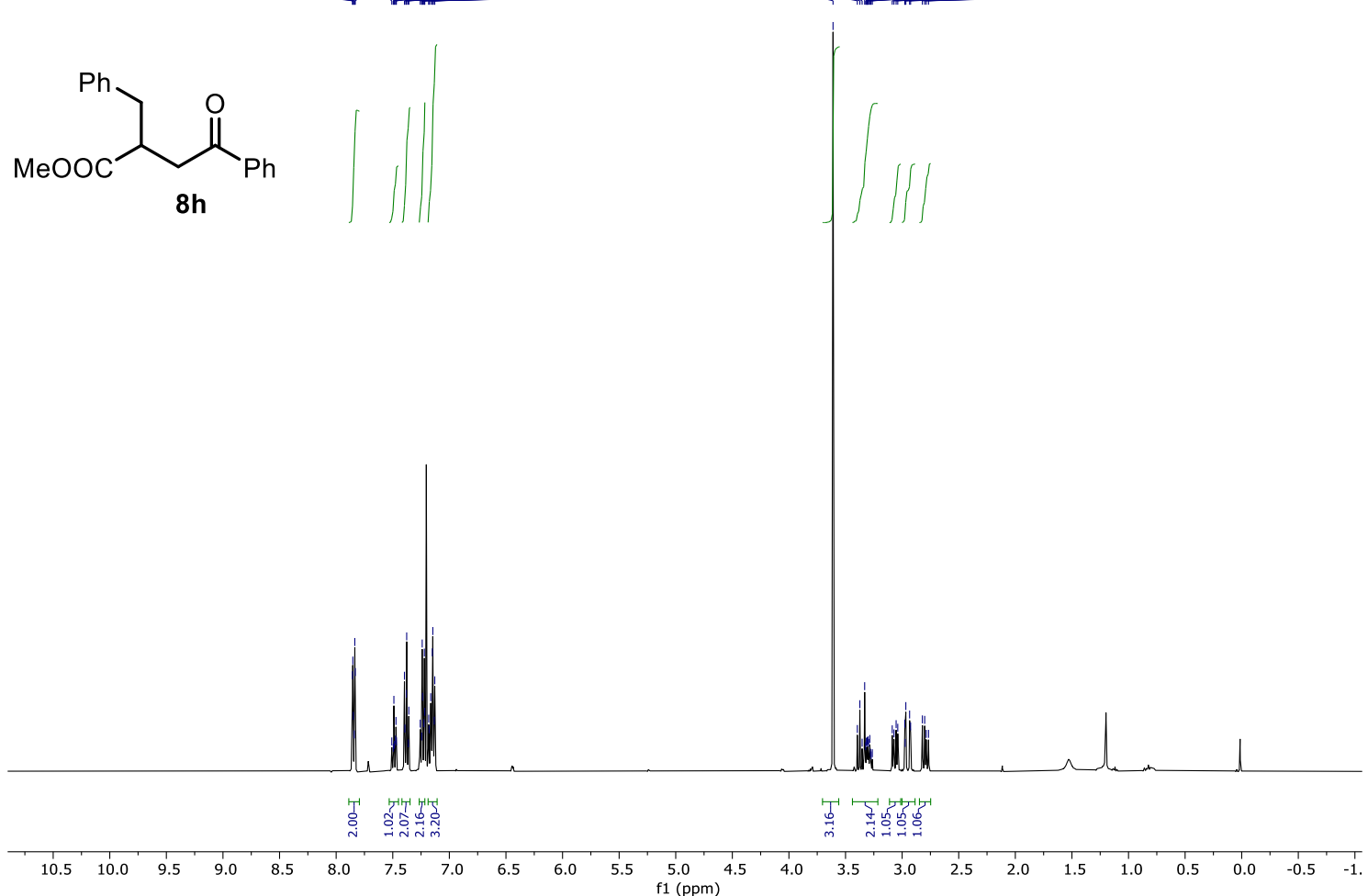

${ }^{13} \mathrm{C}$ NMR $\left(101 \mathrm{MHz}, \mathrm{CDCl}_{3}\right)$

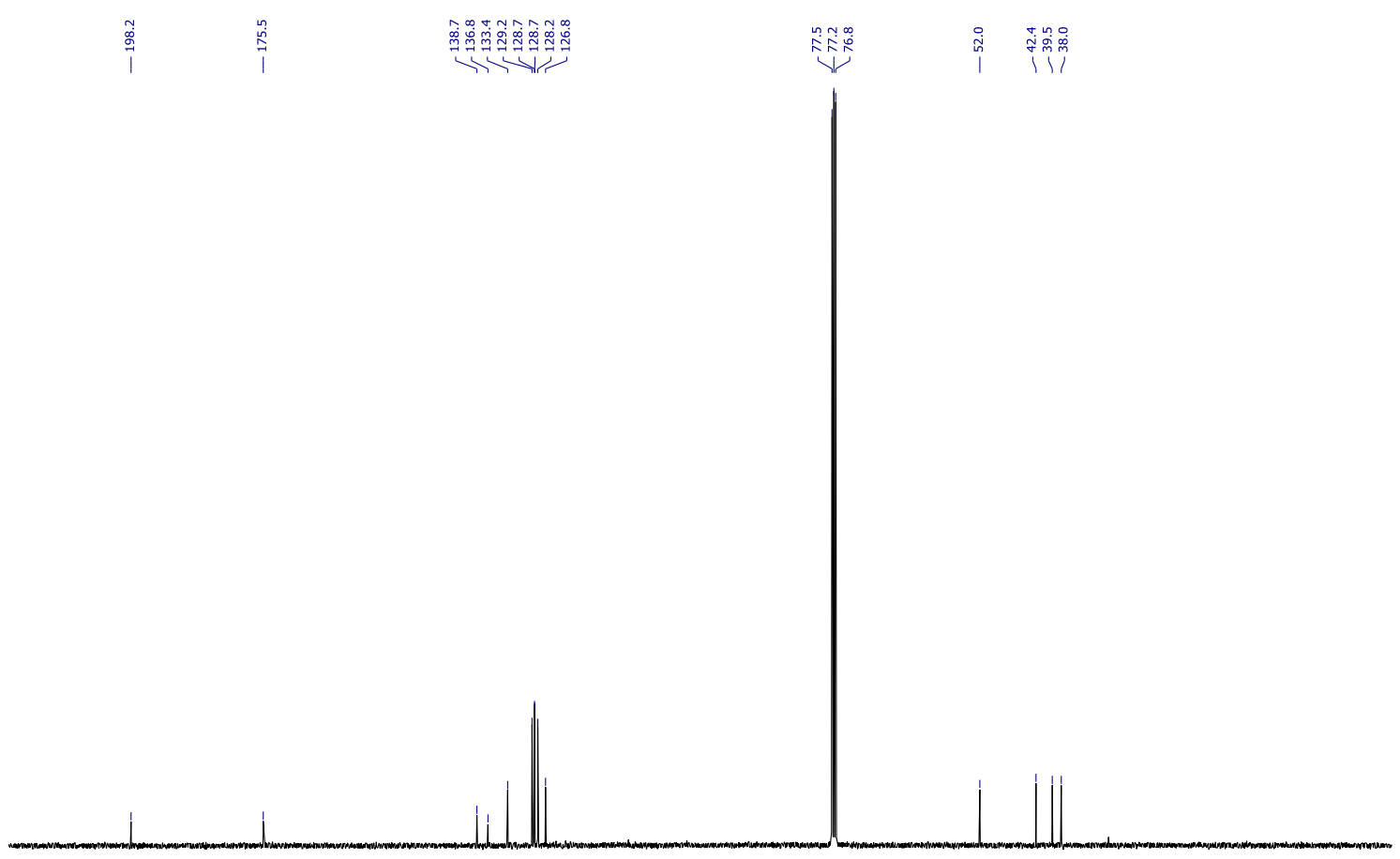

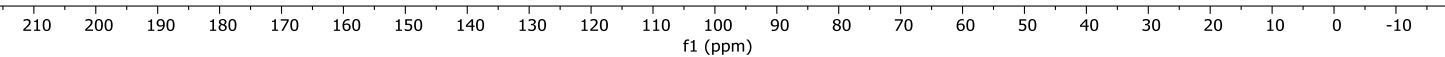


${ }^{1} \mathrm{H}$ NMR (400 MHz, $\mathrm{CDCl}_{3}$ )

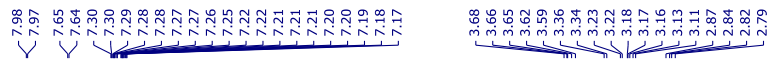<smiles>CC(=O)C(CC(=O)c1cscn1)Cc1ccccc1</smiles>

$8 \mathbf{i}$
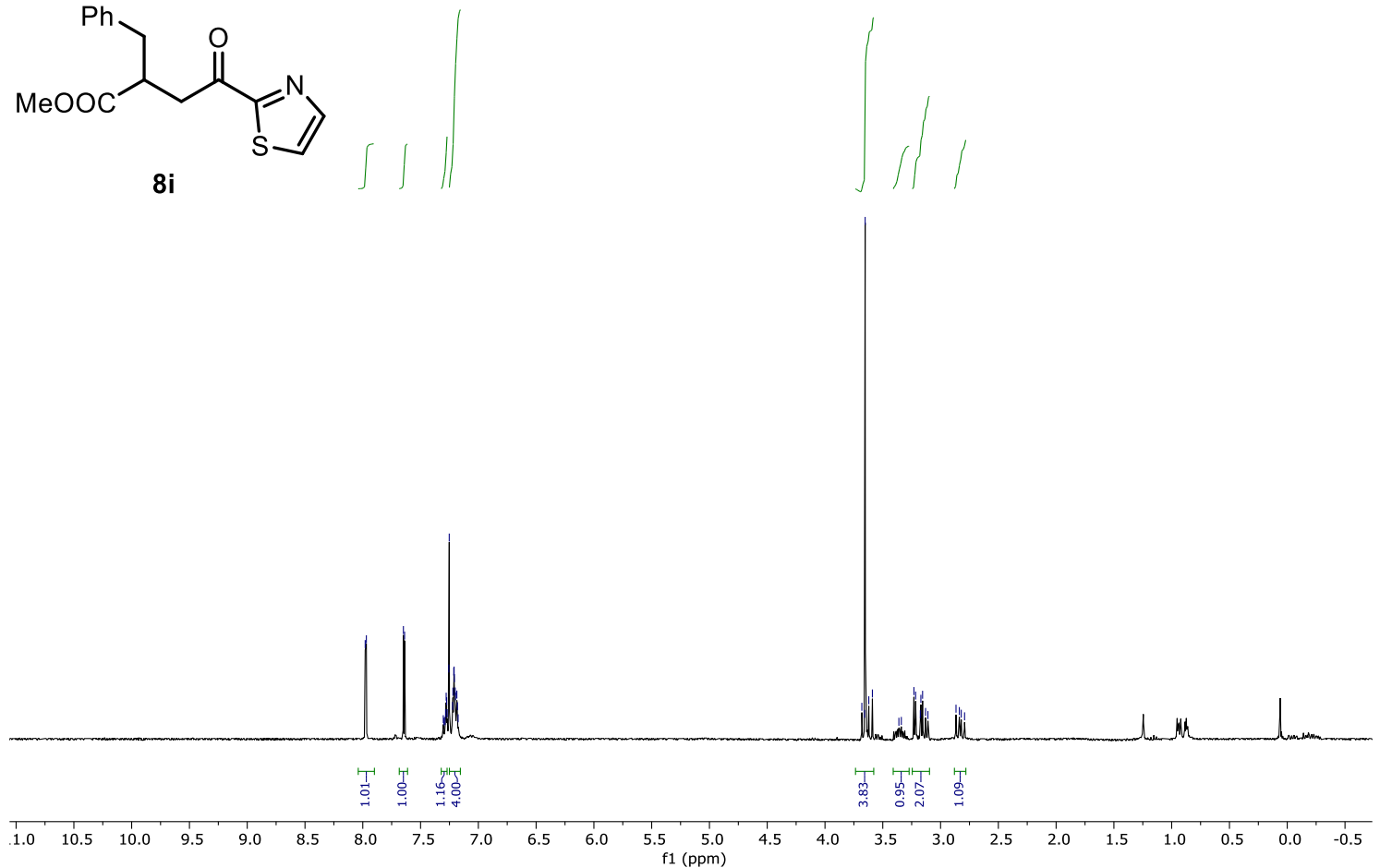

${ }^{13} \mathrm{C}$ NMR $\left(75 \mathrm{MHz}, \mathrm{CDCl}_{3}\right)$

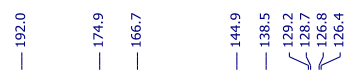


${ }^{1} \mathrm{H}$ NMR (400 MHz, $\mathrm{CDCl}_{3}$ )

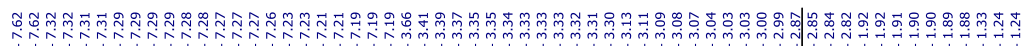<smiles>CC(=O)C(CC(=O)c1cc(C(C)(C)C)cc2c1CCC2(C)C)Cc1ccccc1</smiles>

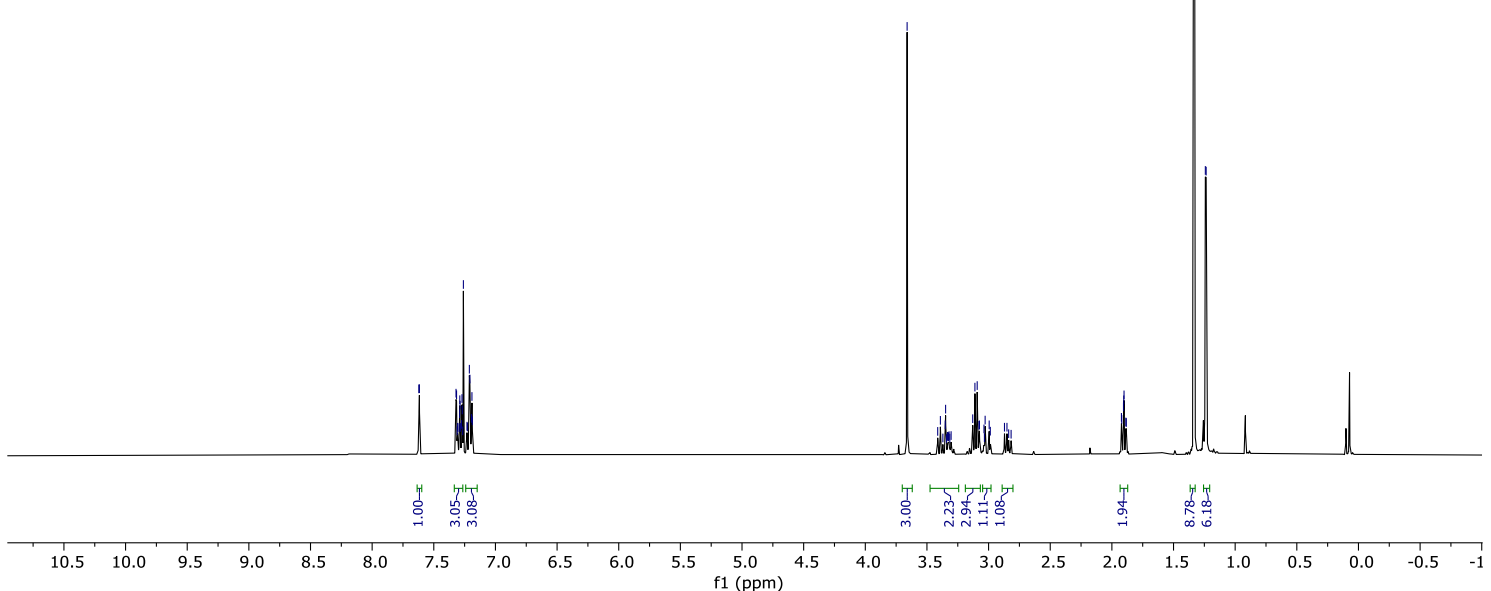

${ }^{13} \mathrm{C}$ NMR $\left(101 \mathrm{MHz}, \mathrm{CDCl}_{3}\right)$

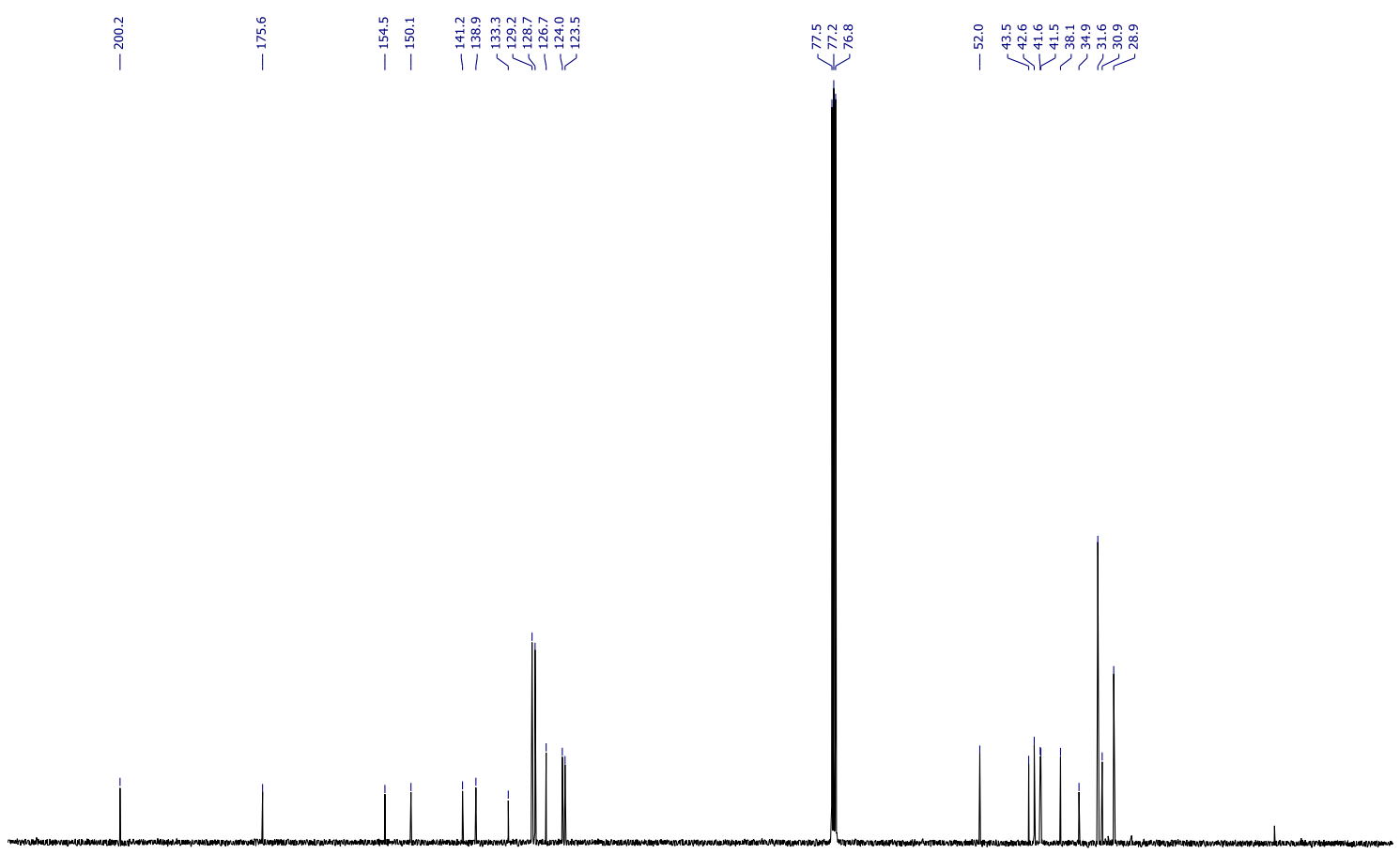

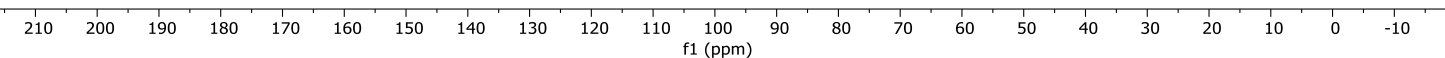


${ }^{1} \mathrm{H}$ NMR (400 MHz, $\mathrm{CDCl}_{3}$ )

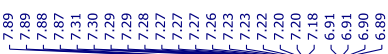

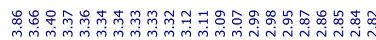<smiles>COC(=O)C(CC(=O)c1ccc(OC)cc1)Cc1ccccc1</smiles>
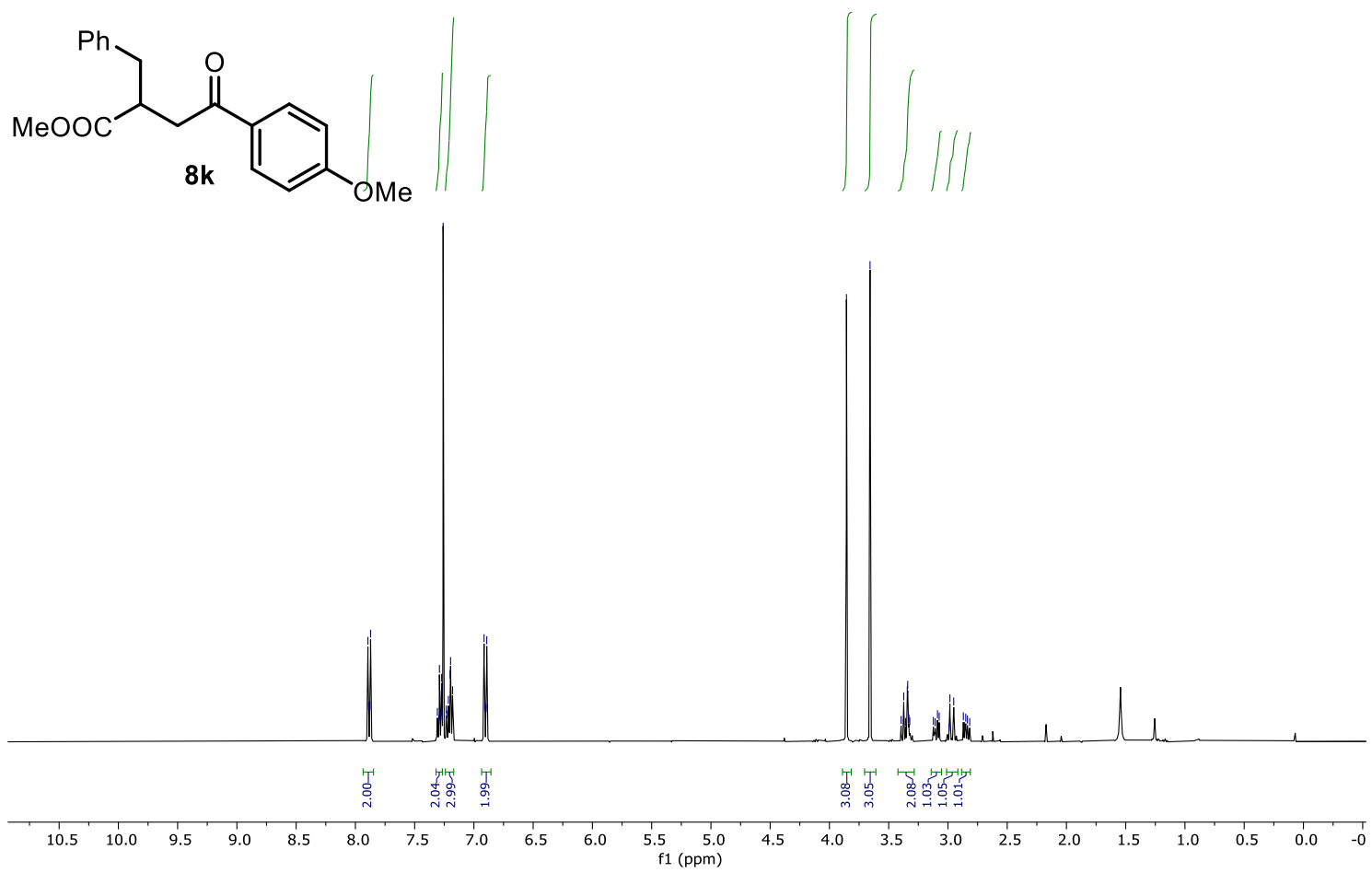

${ }^{13} \mathrm{C}$ NMR (101 MHz, $\left.\mathrm{CDCl}_{3}\right)$

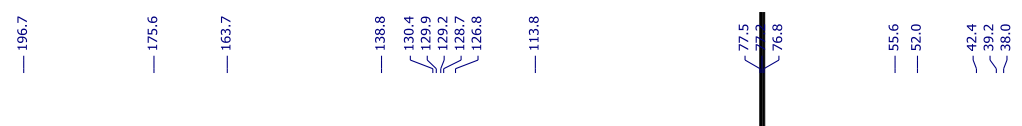

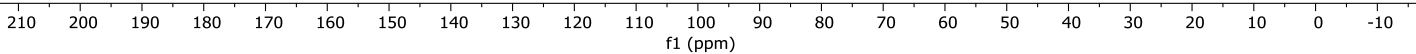


${ }^{1} \mathrm{H}$ NMR (300 MHz, $\mathrm{CDCl}_{3}$ )

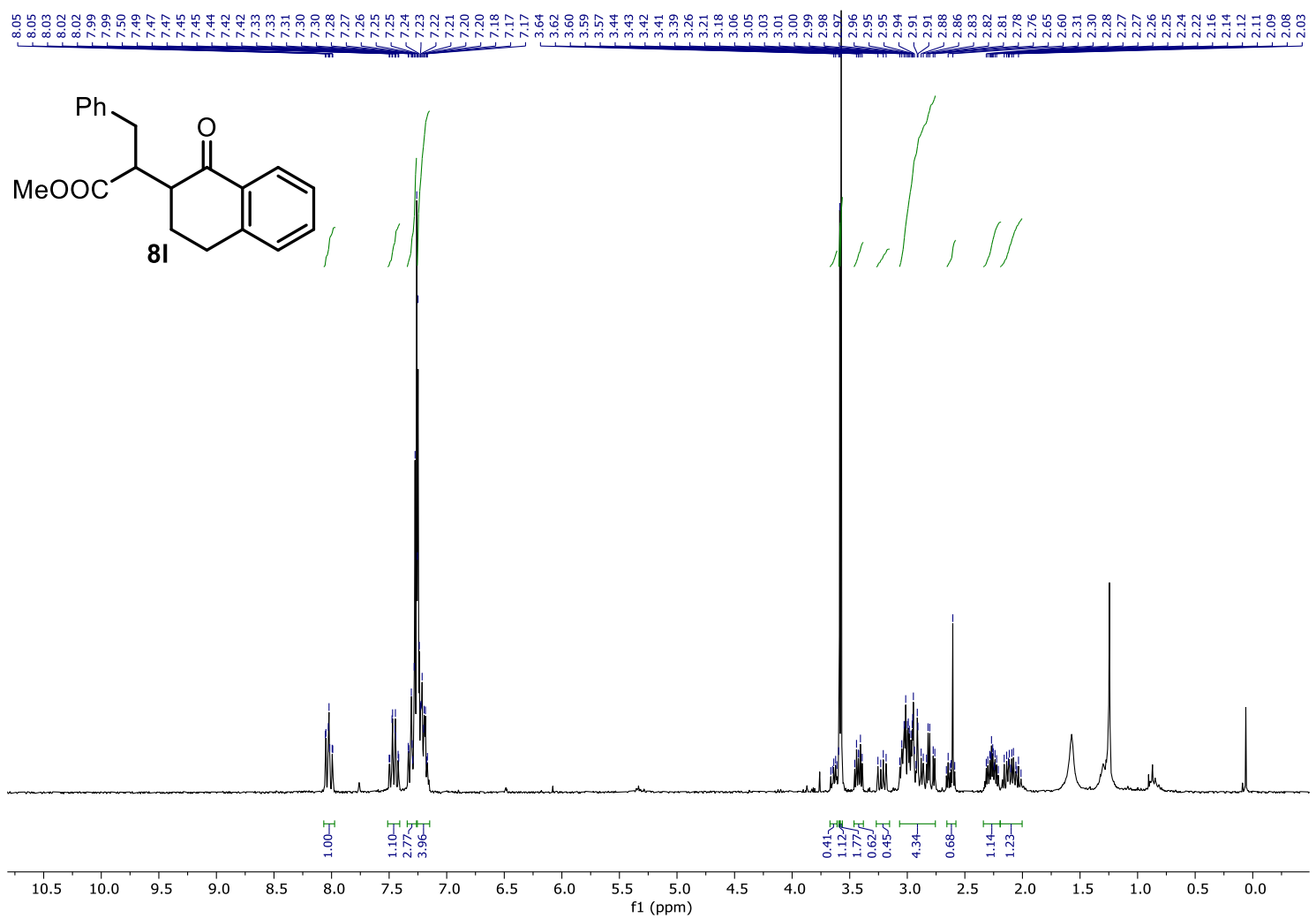

${ }^{13} \mathrm{C}$ NMR $\left(75 \mathrm{MHz}, \mathrm{CDCl}_{3}\right)$

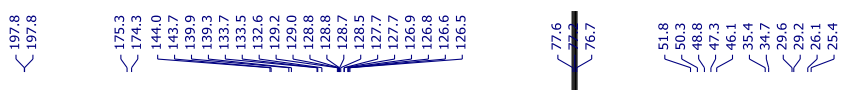

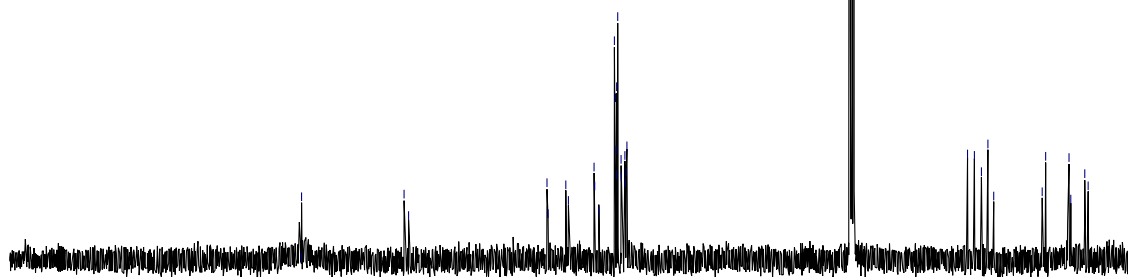

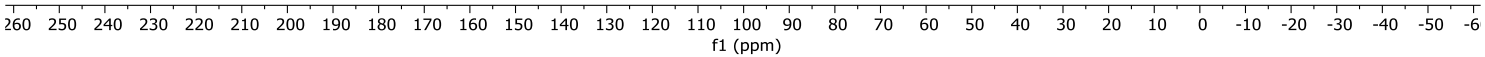


${ }^{1} \mathrm{H}$ NMR (500 MHz, $\mathrm{CDCl}_{3}$ )

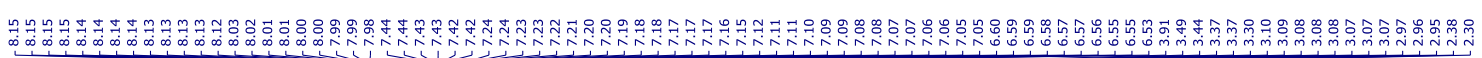

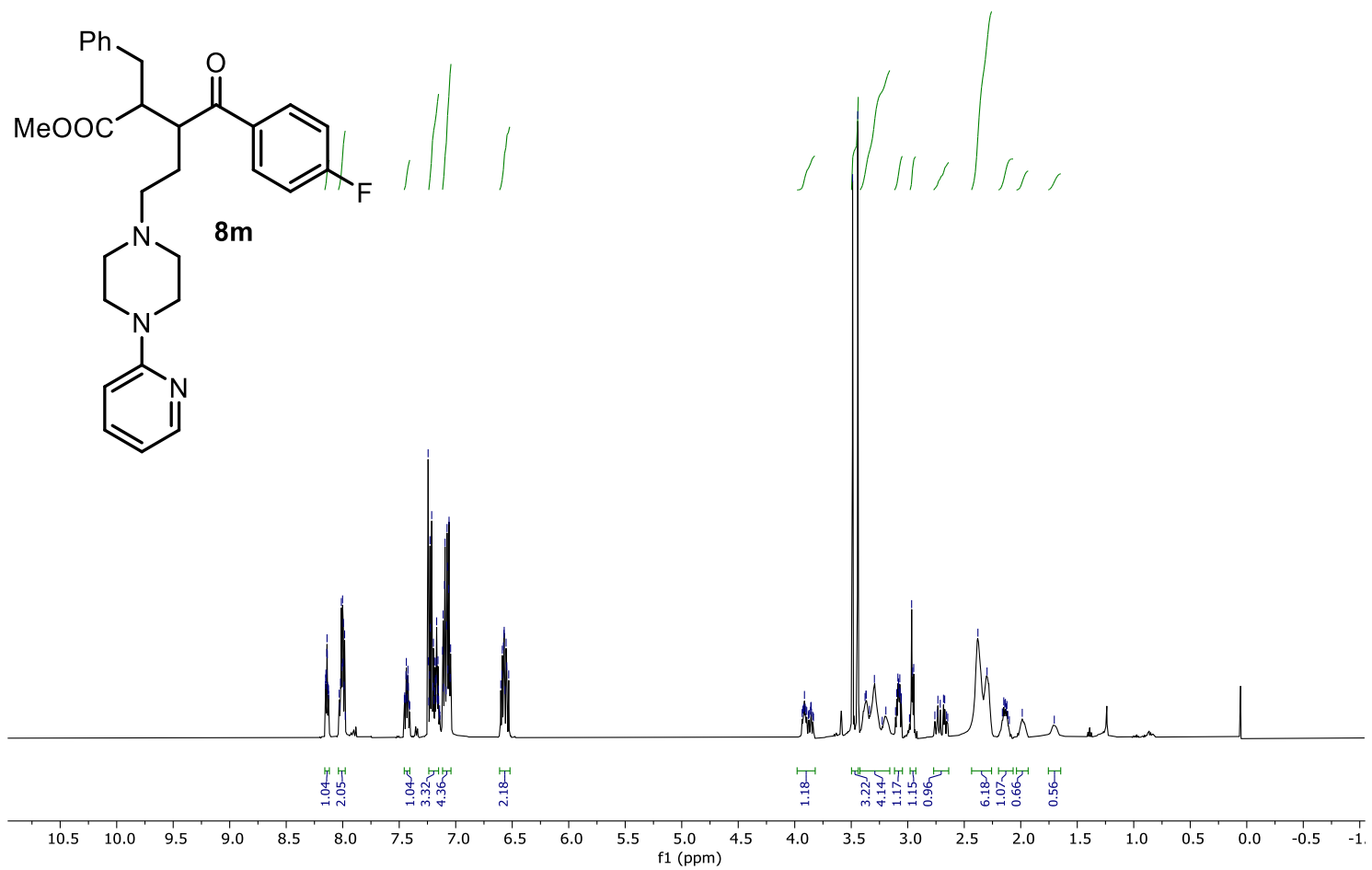

${ }^{13} \mathrm{C} \mathrm{NMR}\left(126 \mathrm{MHz}, \mathrm{CDCl}_{3}\right)$

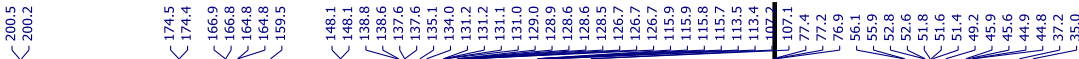

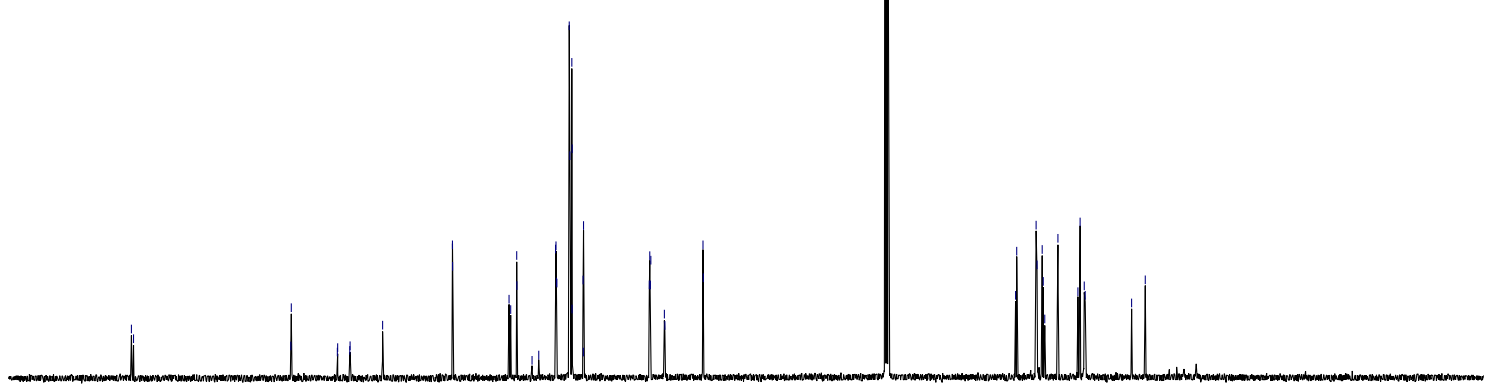

$\begin{array}{lllllllllllllllllllllllllllll}120 & 210 & 200 & 190 & 180 & 170 & 160 & 150 & 140 & 130 & 120 & 110 & 100 & 90 & 80 & 70 & 60 & 50 & 40 & 30 & 20 & 10 & 0 & -10 & -2\end{array}$ 
${ }^{19} \mathrm{~F}$ NMR $\left(376 \mathrm{MHz}, \mathrm{CDCl}_{3}\right)$

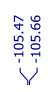

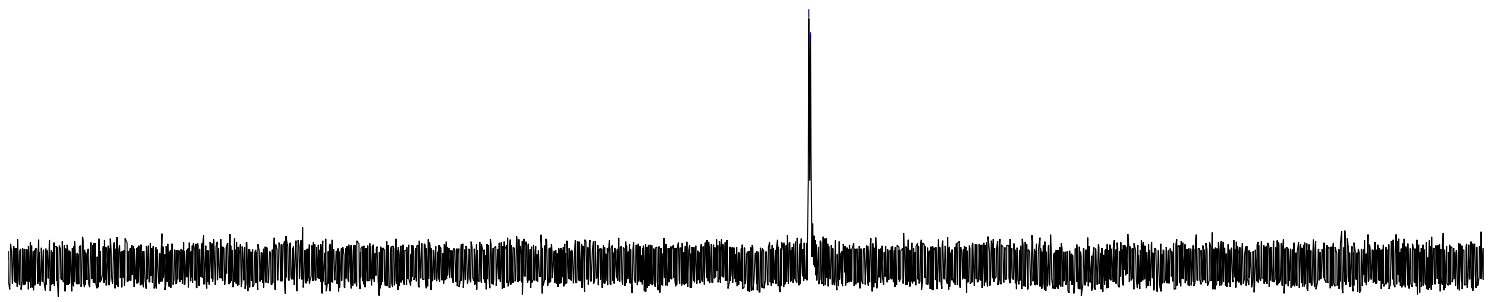

\begin{tabular}{|lllllllllllllllll}
\hline-20 & -30 & -40 & -50 & -60 & -70 & -80 & -90 & $\begin{array}{r}1 \\
\mathrm{f} 1(\mathrm{ppm})\end{array}$ & -110 & -120 & -130 & -140 & -150 & -160 & -170 & -180 \\
\hline
\end{tabular}


${ }^{1} \mathrm{H}$ NMR (400 MHz, $\mathrm{CDCl}_{3}$ )

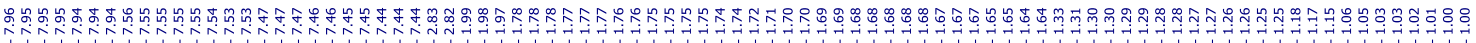<smiles>O=C(CC1CCCCC1)c1ccccc1</smiles>

$8 n$
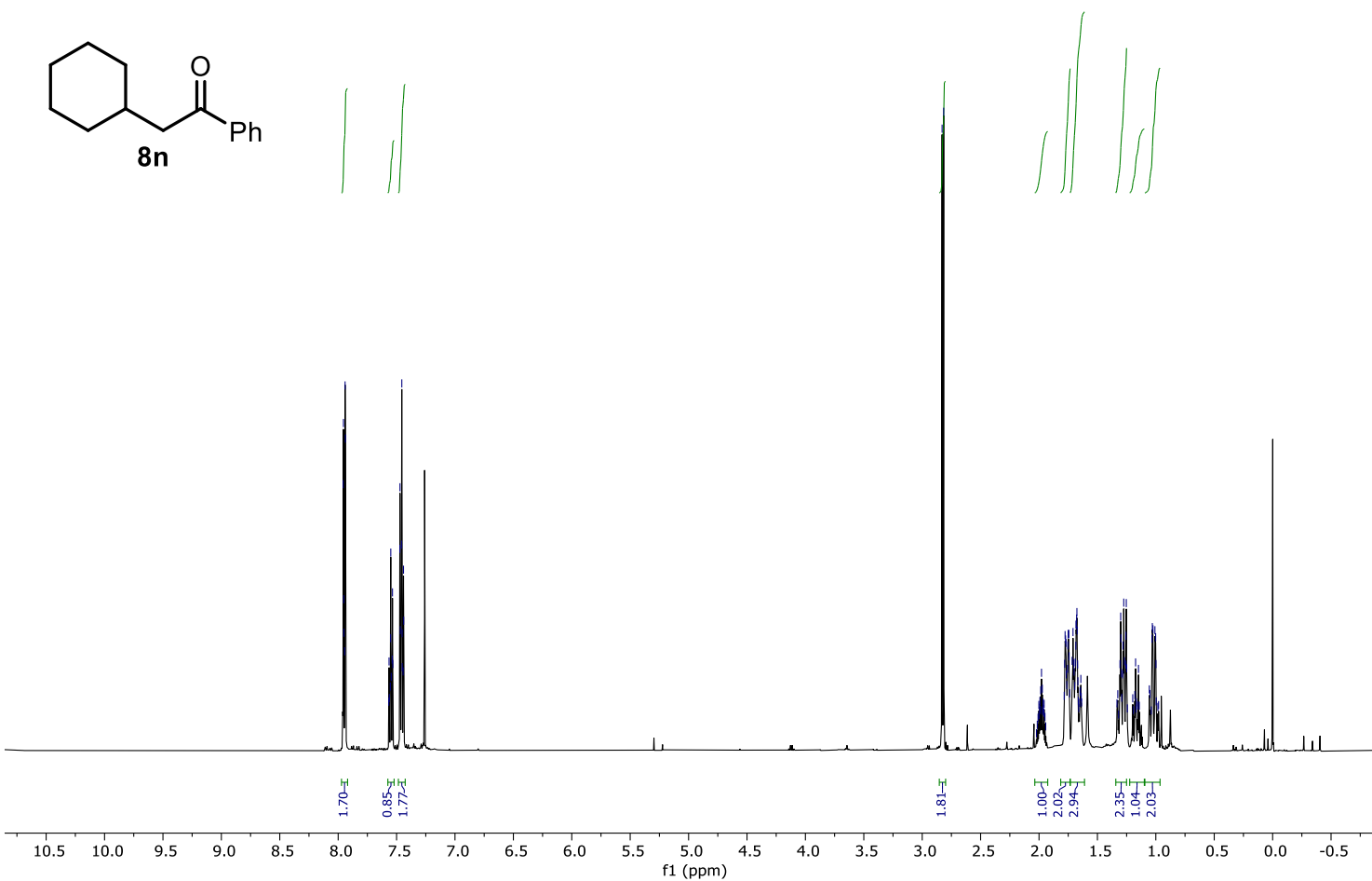

${ }^{13} \mathrm{C} \mathrm{NMR}\left(101 \mathrm{MHz}, \mathrm{CDCl}_{3}\right)$

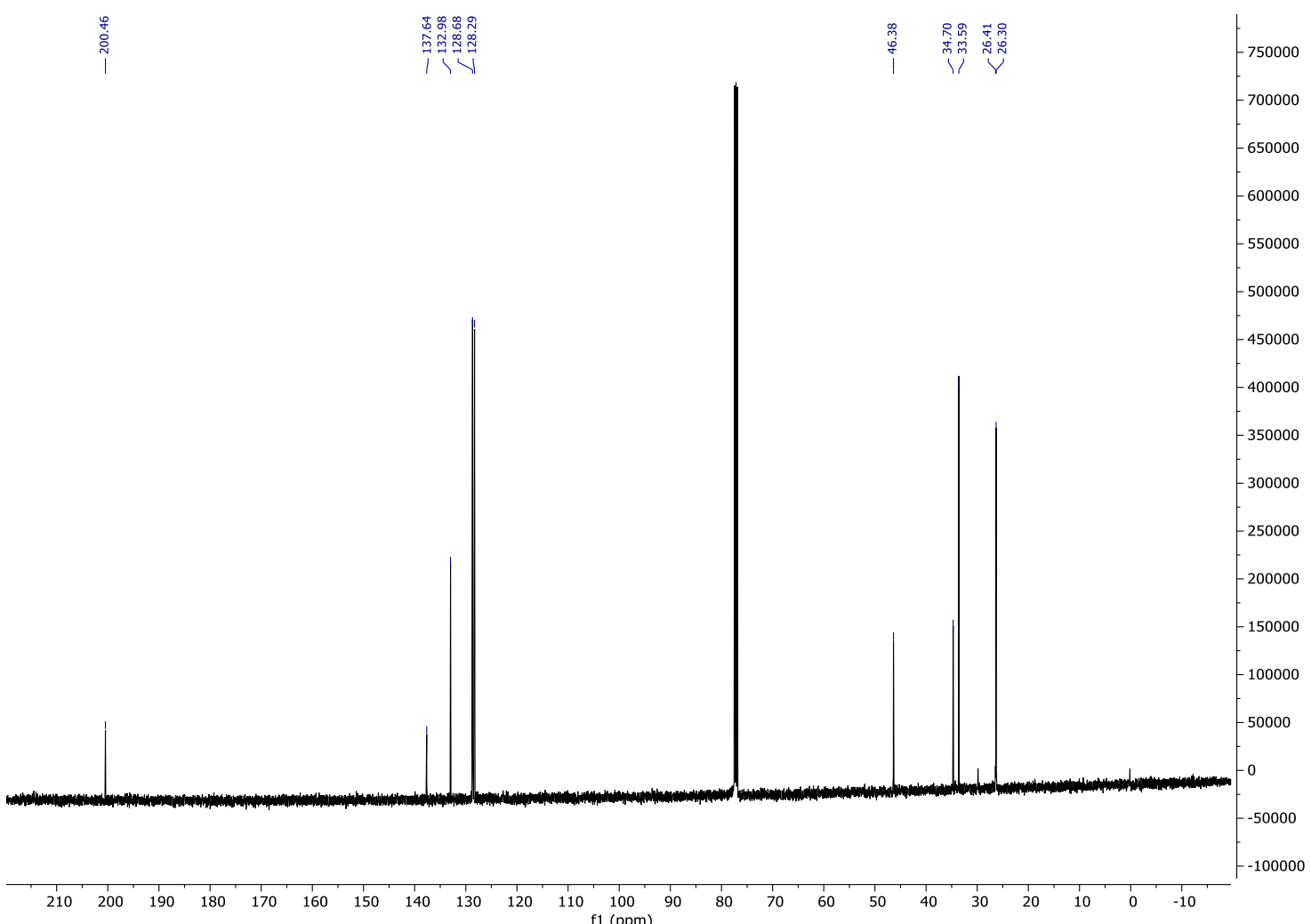


${ }^{1} \mathrm{H}$ NMR (400 MHz, $\left.\mathrm{CDCl}_{3}\right)$

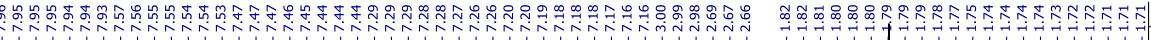

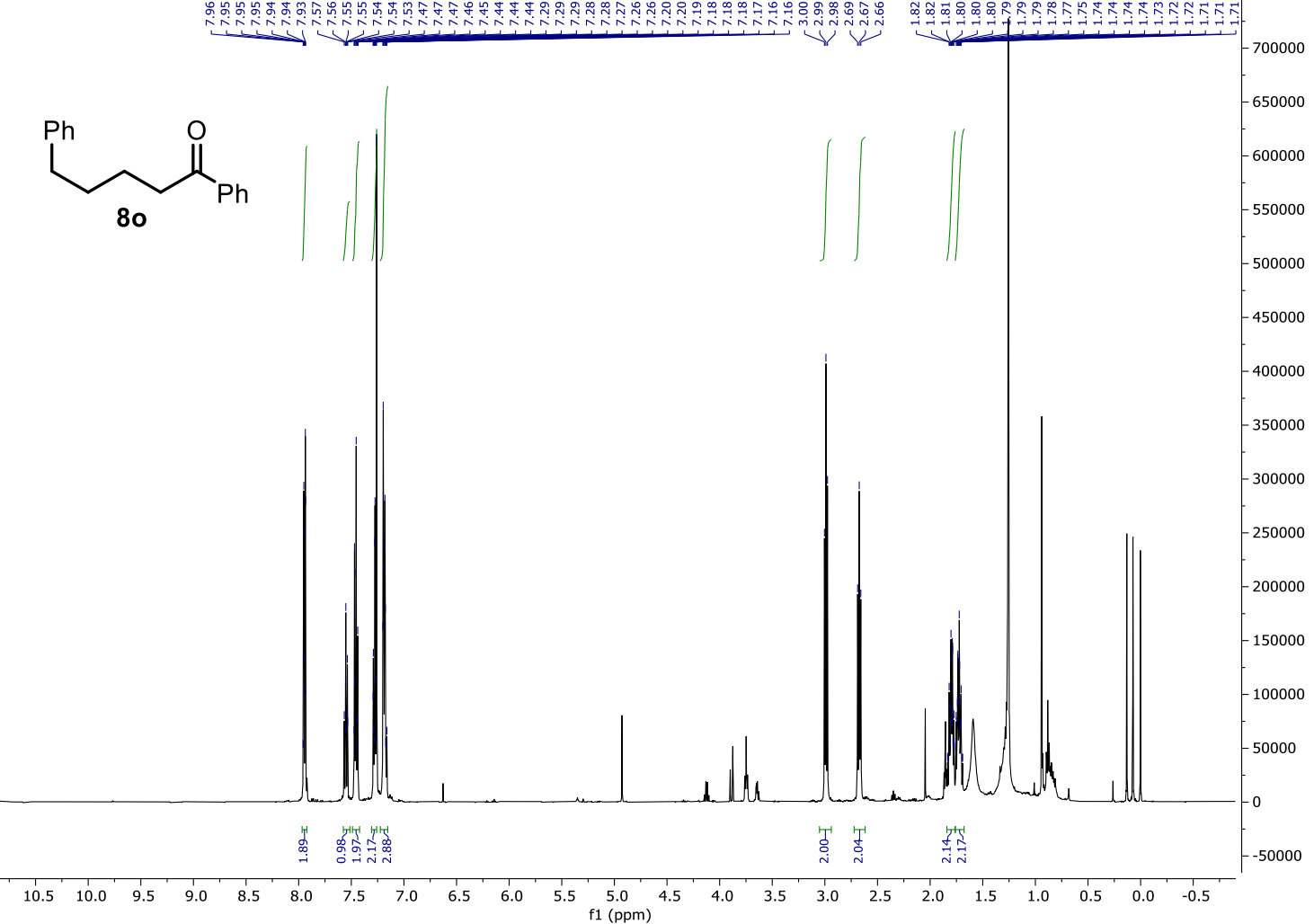

${ }^{13} \mathrm{C}$ NMR (101 MHz, $\left.\mathrm{CDCl}_{3}\right)$

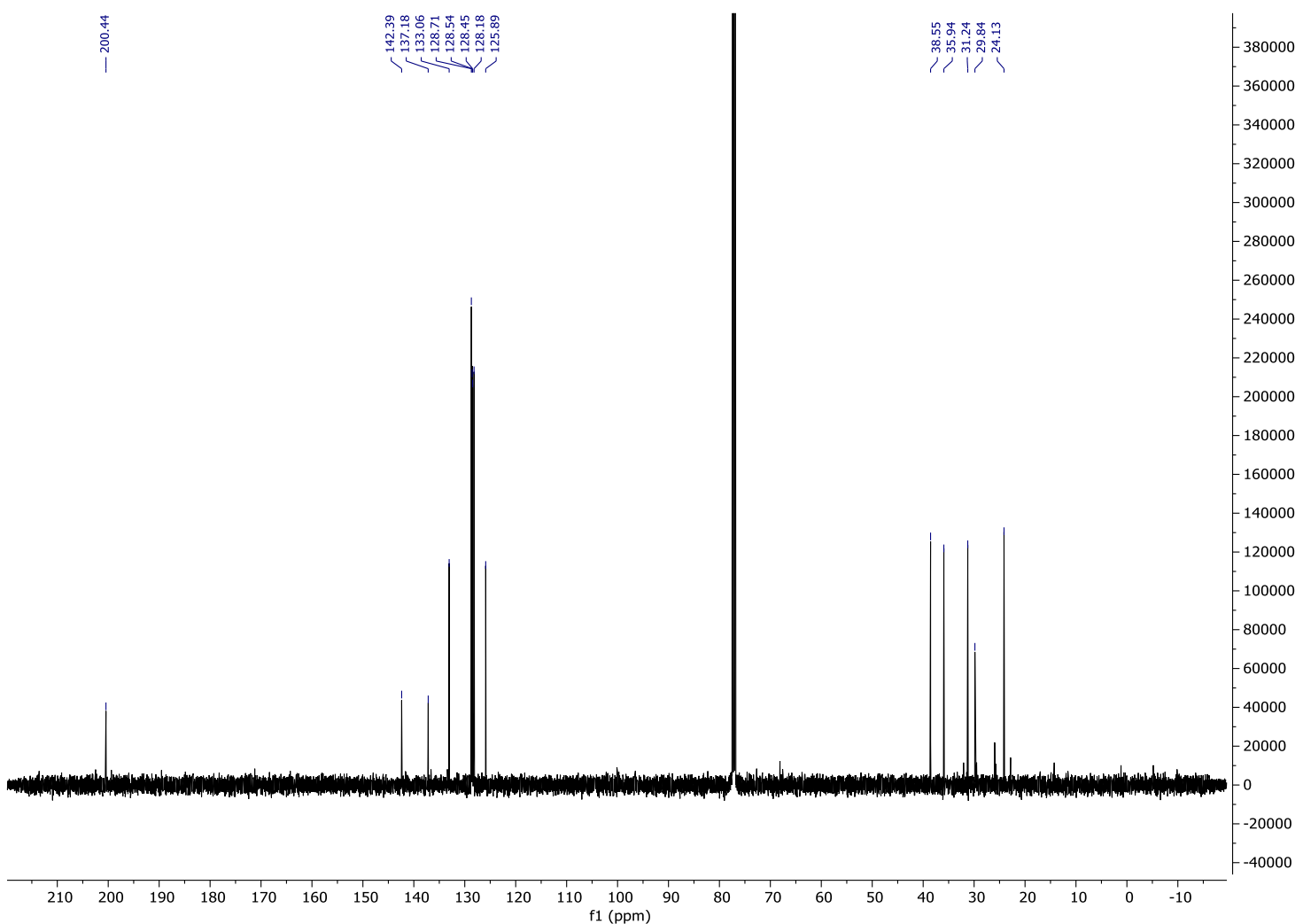


${ }^{1} \mathrm{H}$ NMR (300 MHz, $\left.\mathrm{CDCl}_{3}\right)$

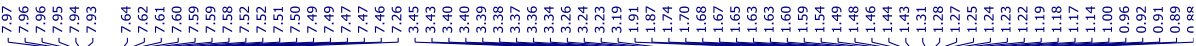<smiles>CC(C[CH]Cl)CC(=O)c1ccccc1</smiles>

9a
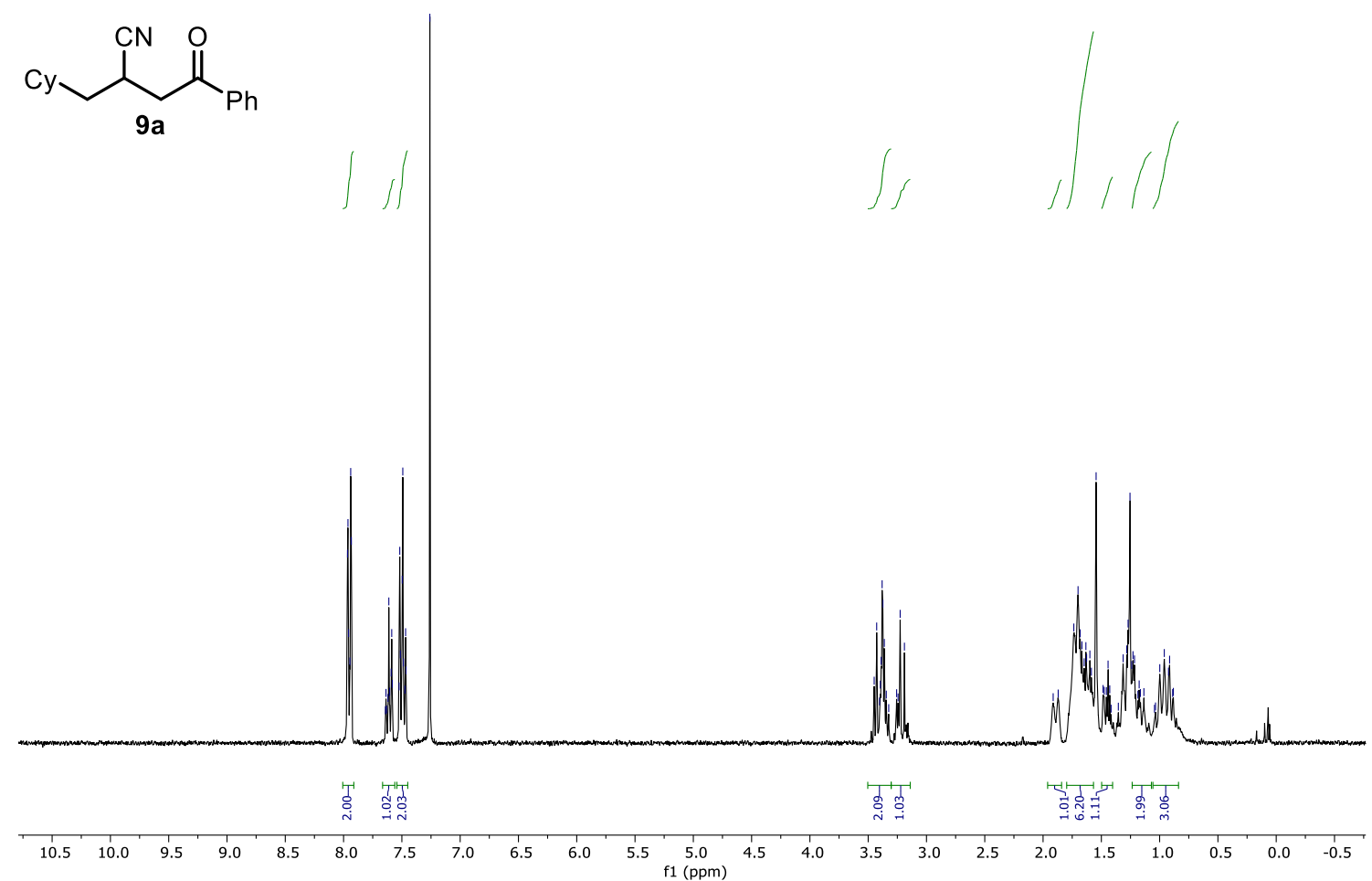

${ }^{13} \mathrm{C} \mathrm{NMR}\left(75 \mathrm{MHz}, \mathrm{CDCl}_{3}\right)$

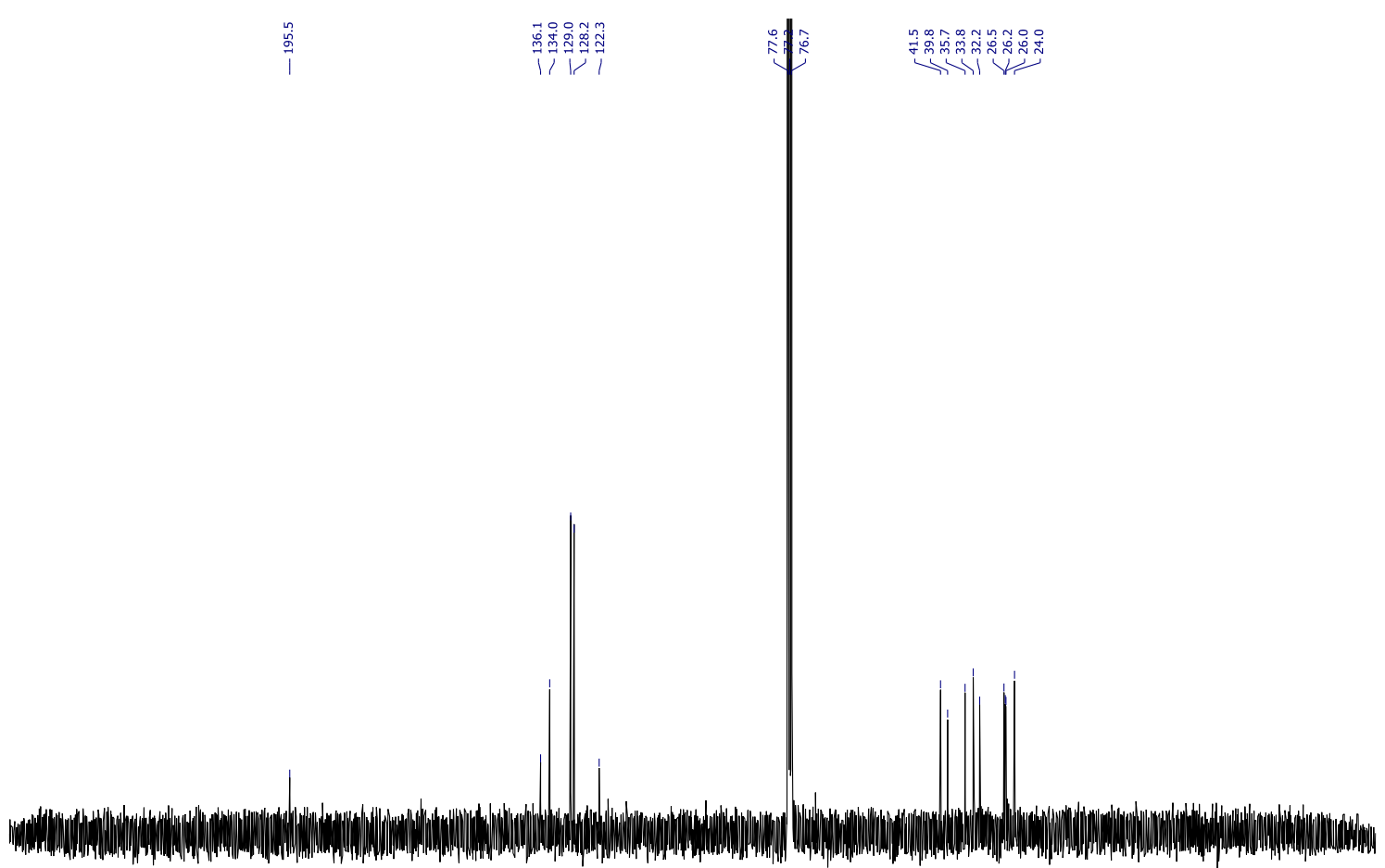

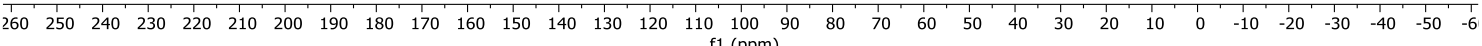


${ }^{1} \mathrm{H}$ NMR (300 MHz, $\left.\mathrm{CDCl}_{3}\right)$

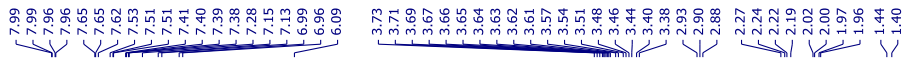
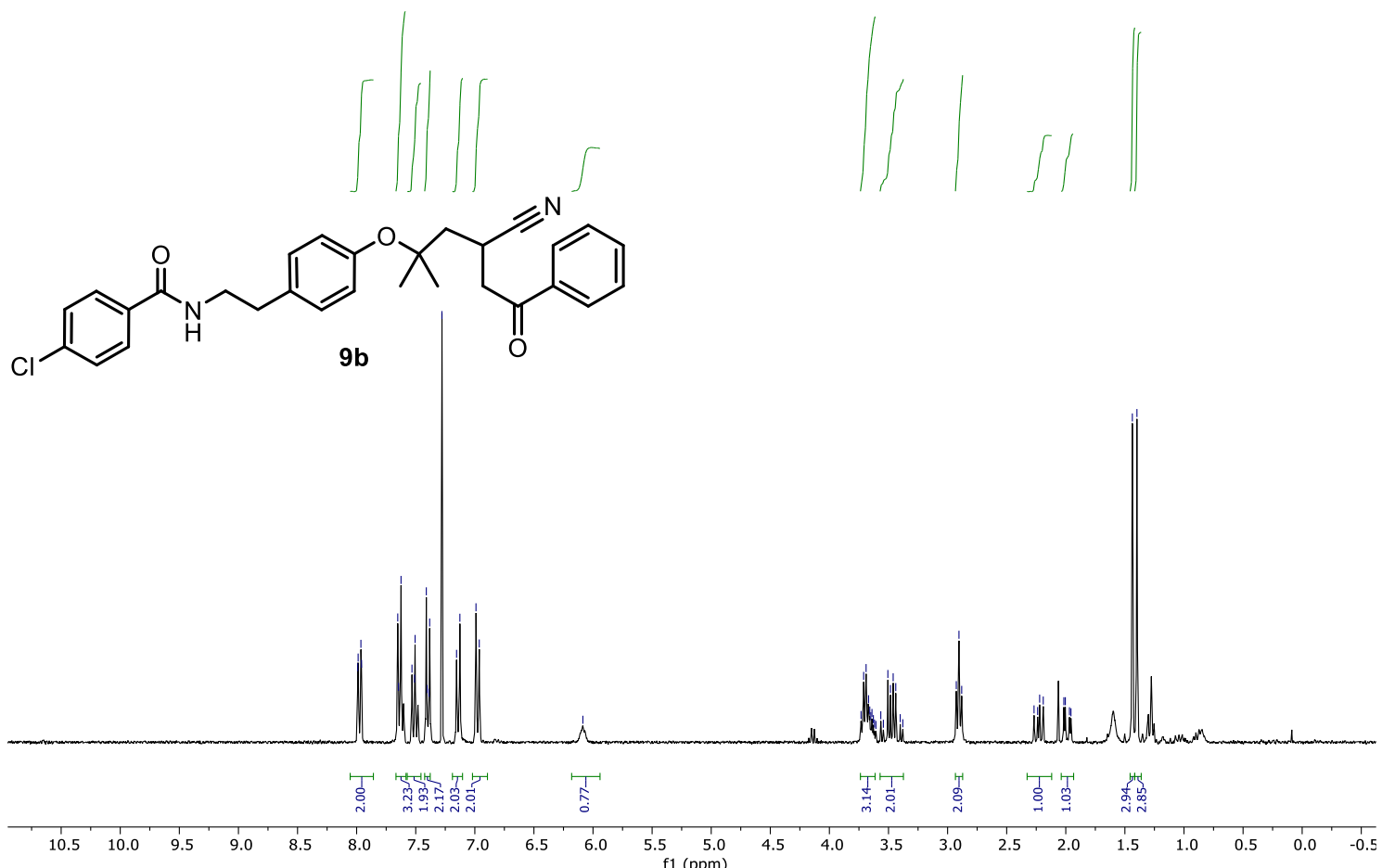

${ }^{13} \mathrm{C}$ NMR $\left(75 \mathrm{MHz}, \mathrm{CDCl}_{3}\right)$

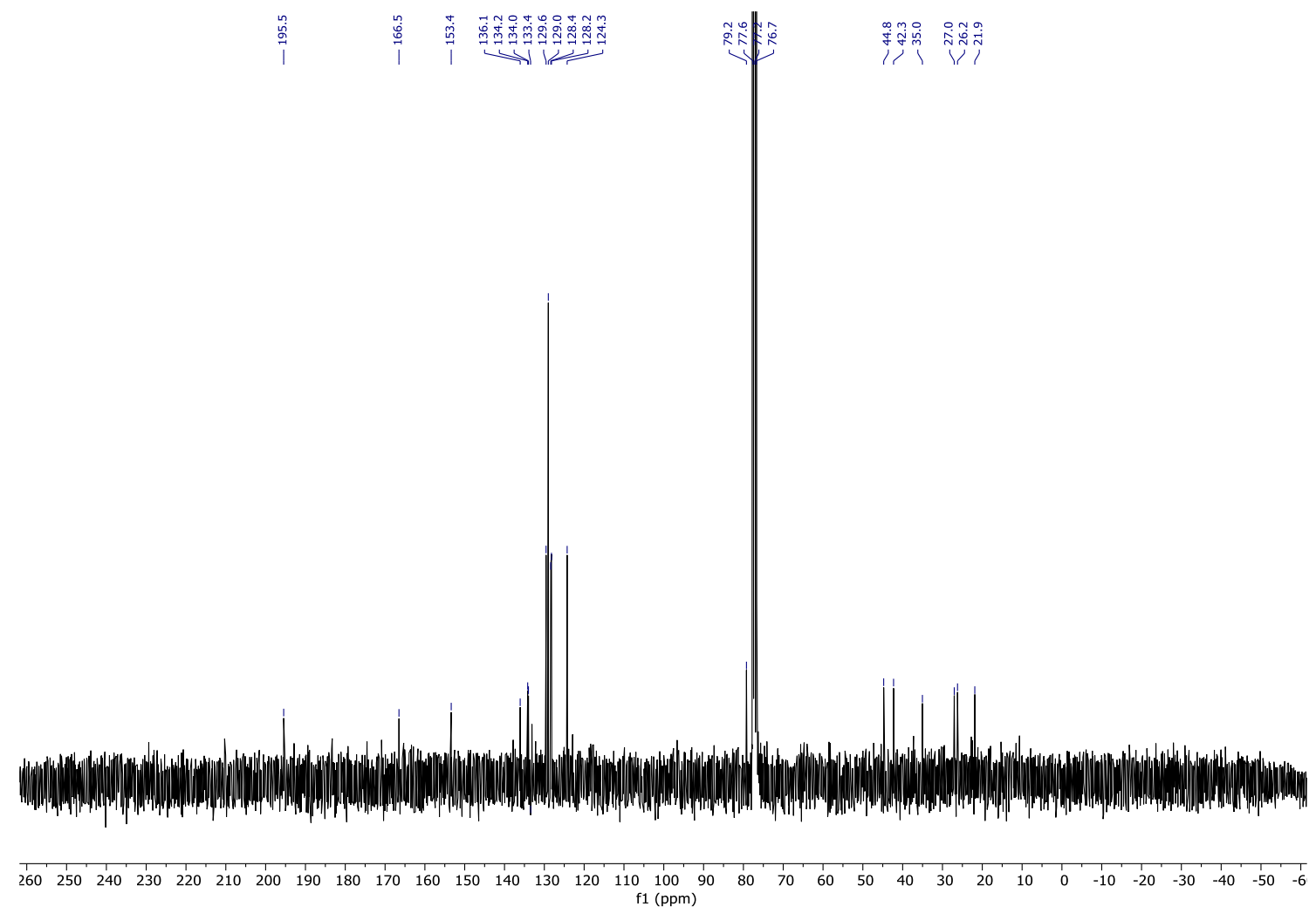


${ }^{1} \mathrm{H}$ NMR (300 MHz, $\mathrm{CDCl}_{3}$ )

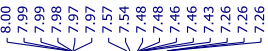<smiles>COC(=O)C(CC(=O)c1ccccc1)C(Cl)C(C)=O</smiles>

9c (Major)

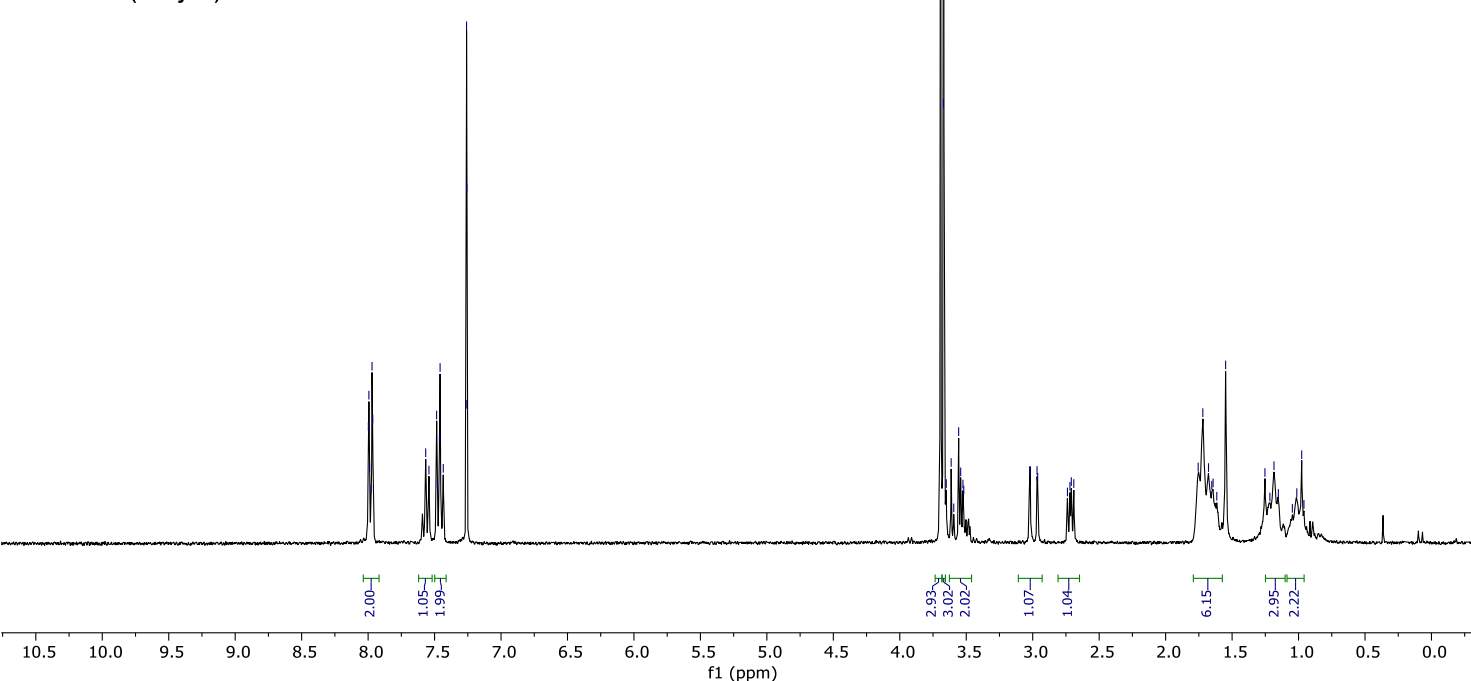

${ }^{13} \mathrm{C}$ NMR $\left(75 \mathrm{MHz}, \mathrm{CDCl}_{3}\right)$

i


${ }^{1} \mathrm{H}$ NMR (300 MHz, $\left.\mathrm{CDCl}_{3}\right)$

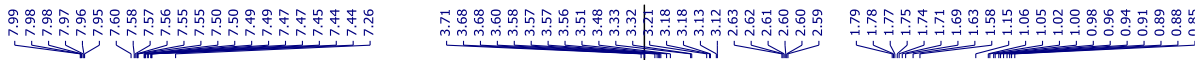<smiles>COC(=O)C(Cl)C(CC(=O)c1ccccc1)C(=O)OC</smiles>

9c (Minor)
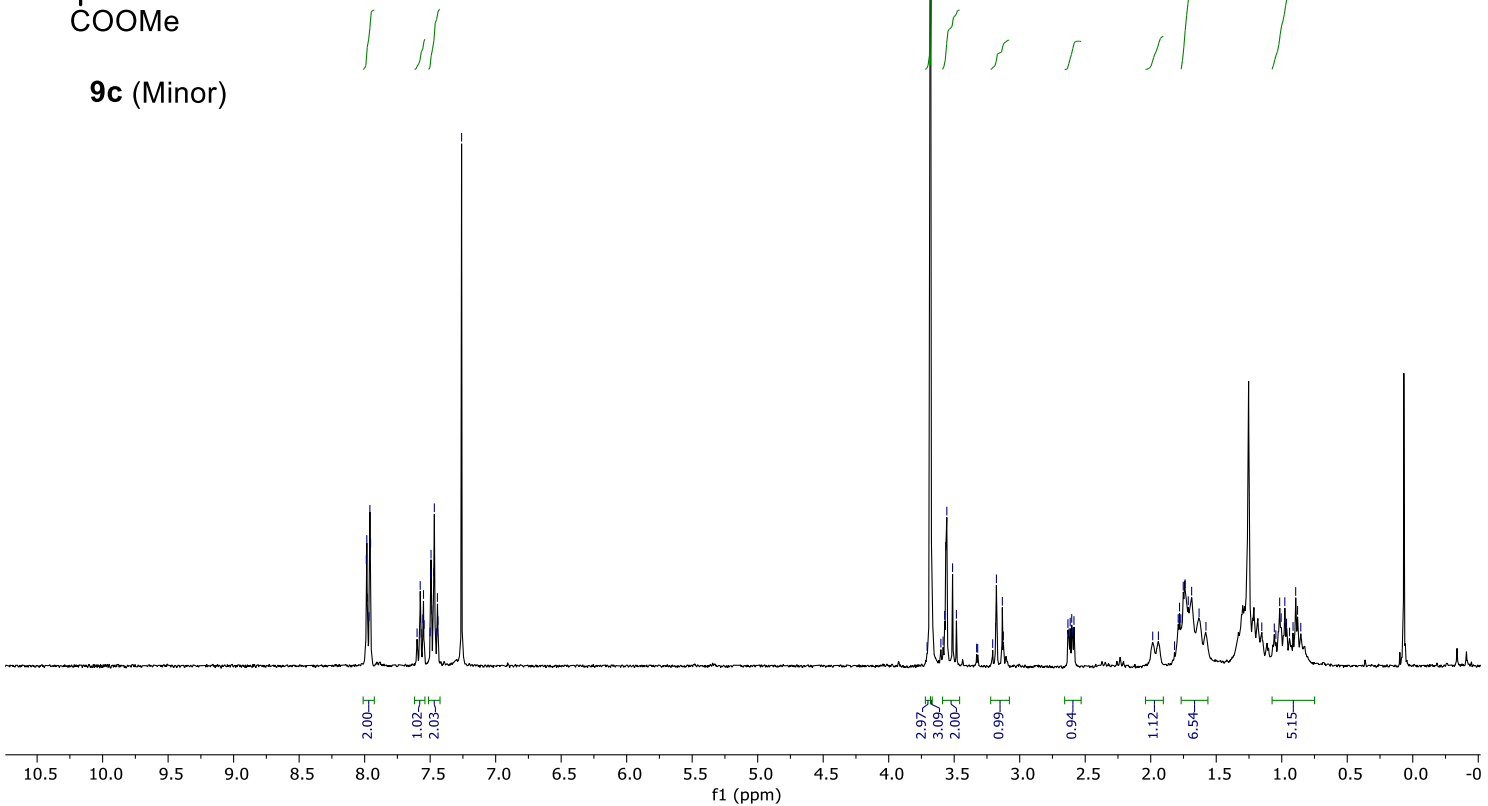

${ }^{13} \mathrm{C}$ NMR $\left(75 \mathrm{MHz}, \mathrm{CDCl}_{3}\right)$

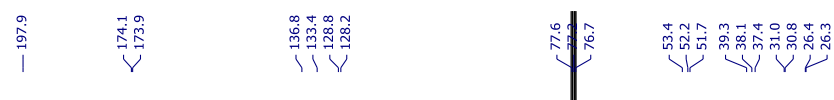

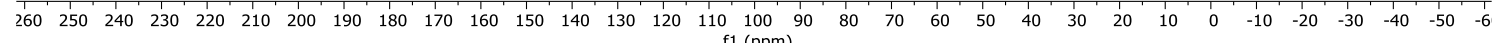
${ }^{1} \mathrm{H}$ NMR $\left(300 \mathrm{MHz}, \mathrm{CDCl}_{3}\right)$ 


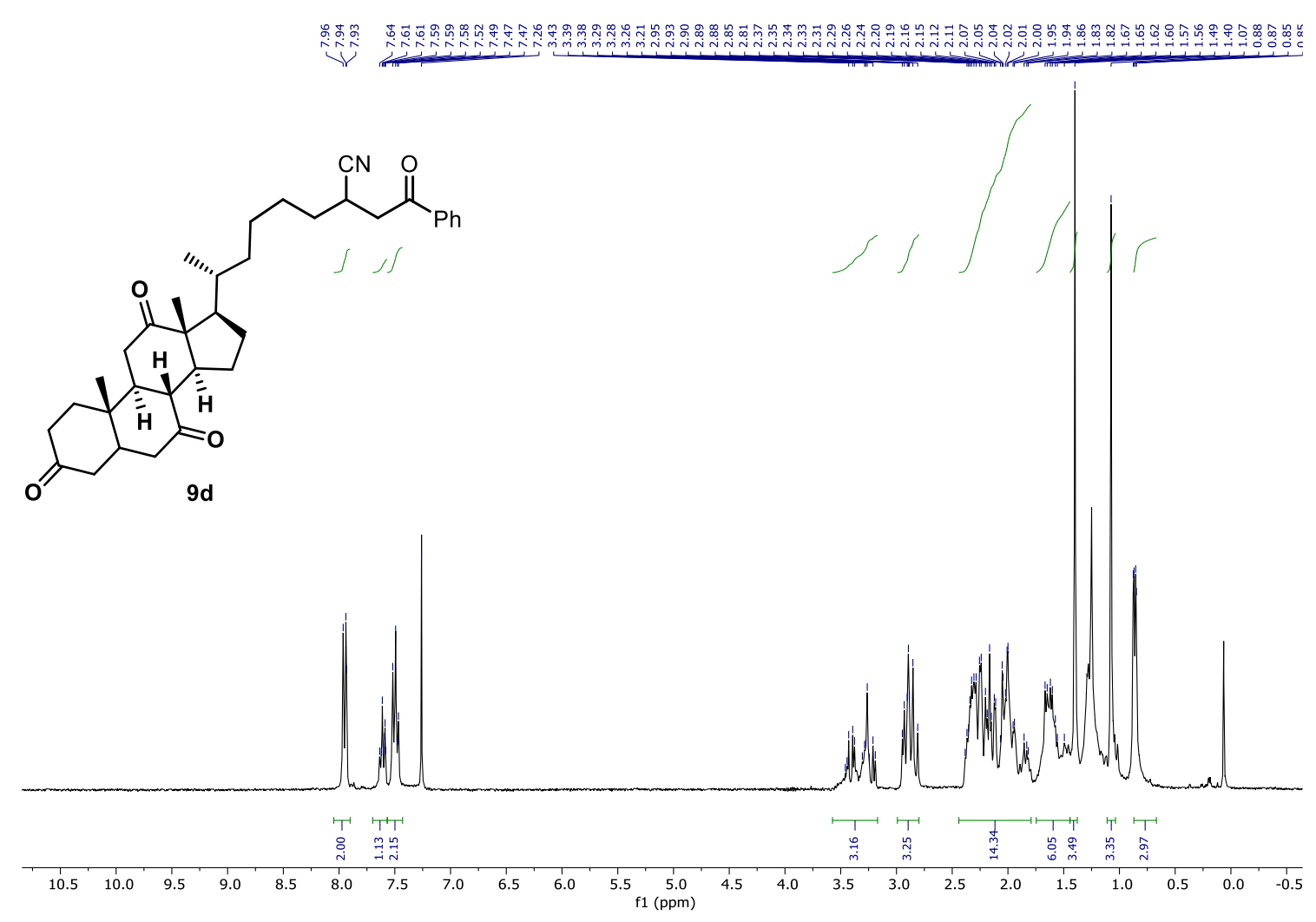

${ }^{13} \mathrm{C} \mathrm{NMR}\left(75 \mathrm{MHz}, \mathrm{CDCl}_{3}\right)$

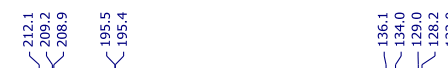

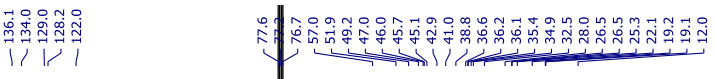

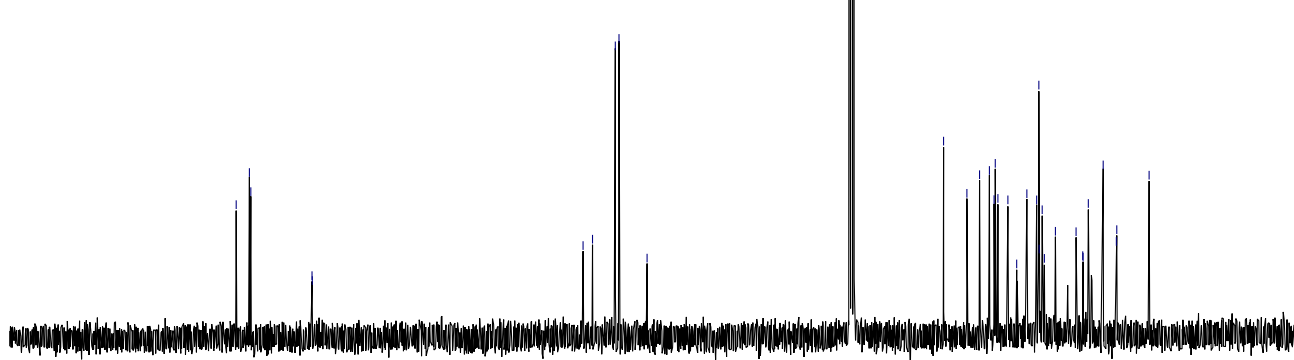

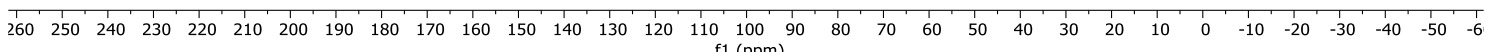


${ }^{1} \mathrm{H}$ NMR (400 MHz, $\mathrm{CDCl}_{3}$ )

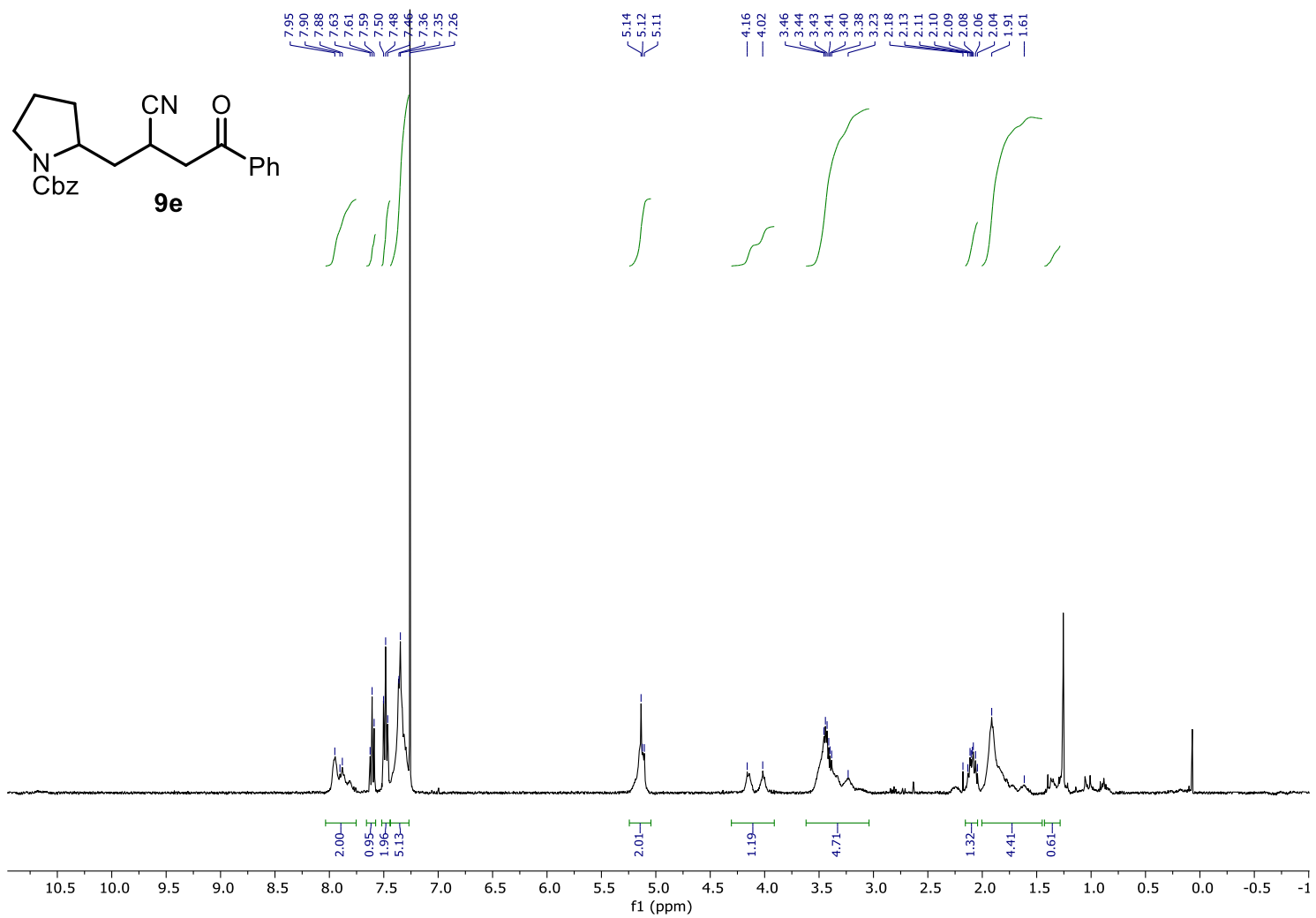

${ }^{13} \mathrm{C}$ NMR (101 MHz, $\left.\mathrm{CDCl}_{3}\right)$

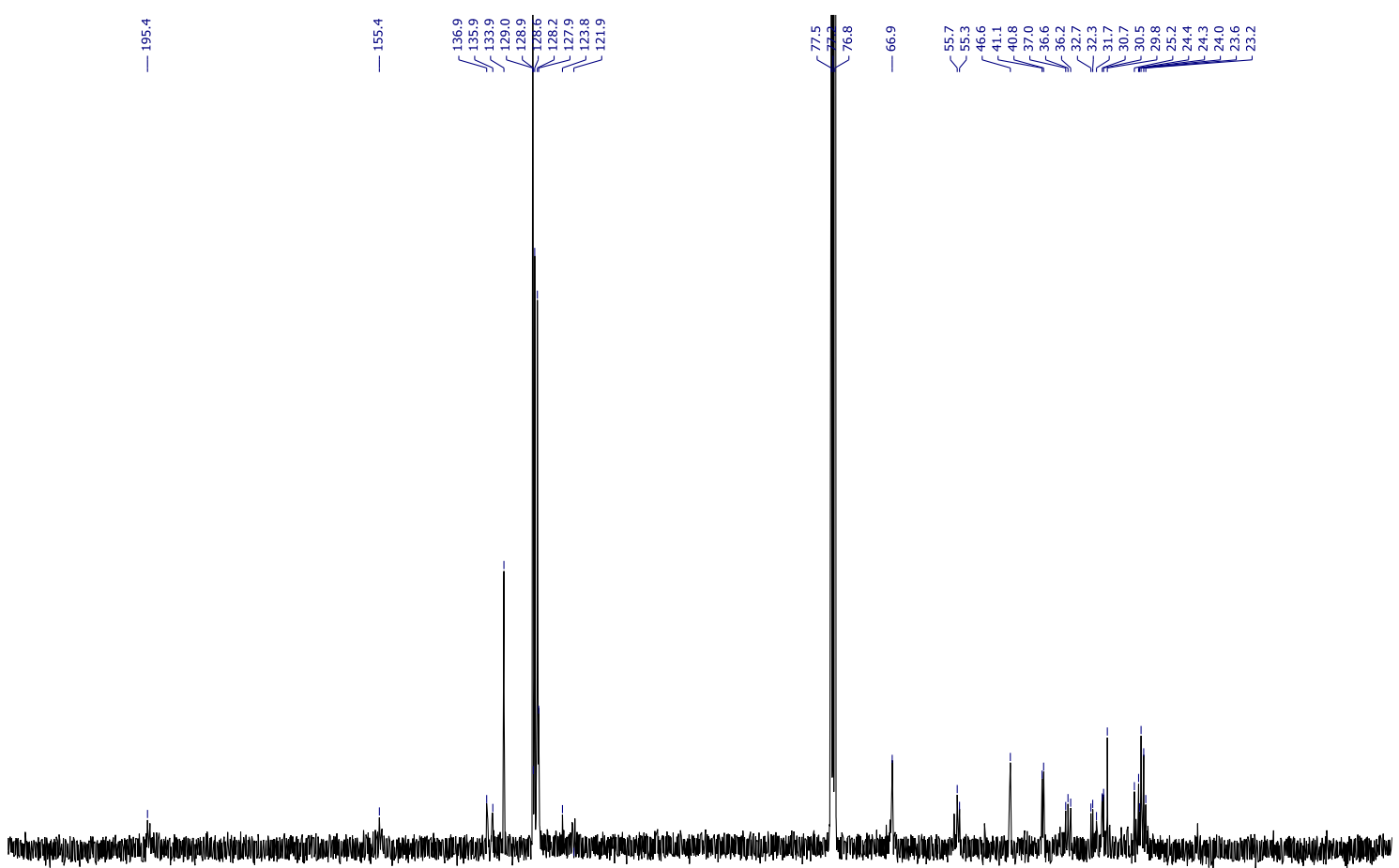

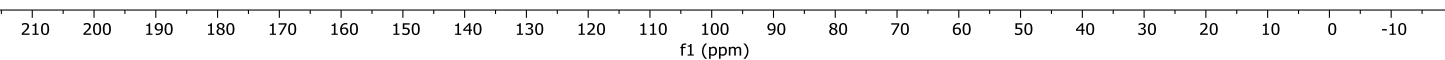


${ }^{1} \mathrm{H}$ NMR (300 MHz, $\mathrm{CDCl}_{3}$ )

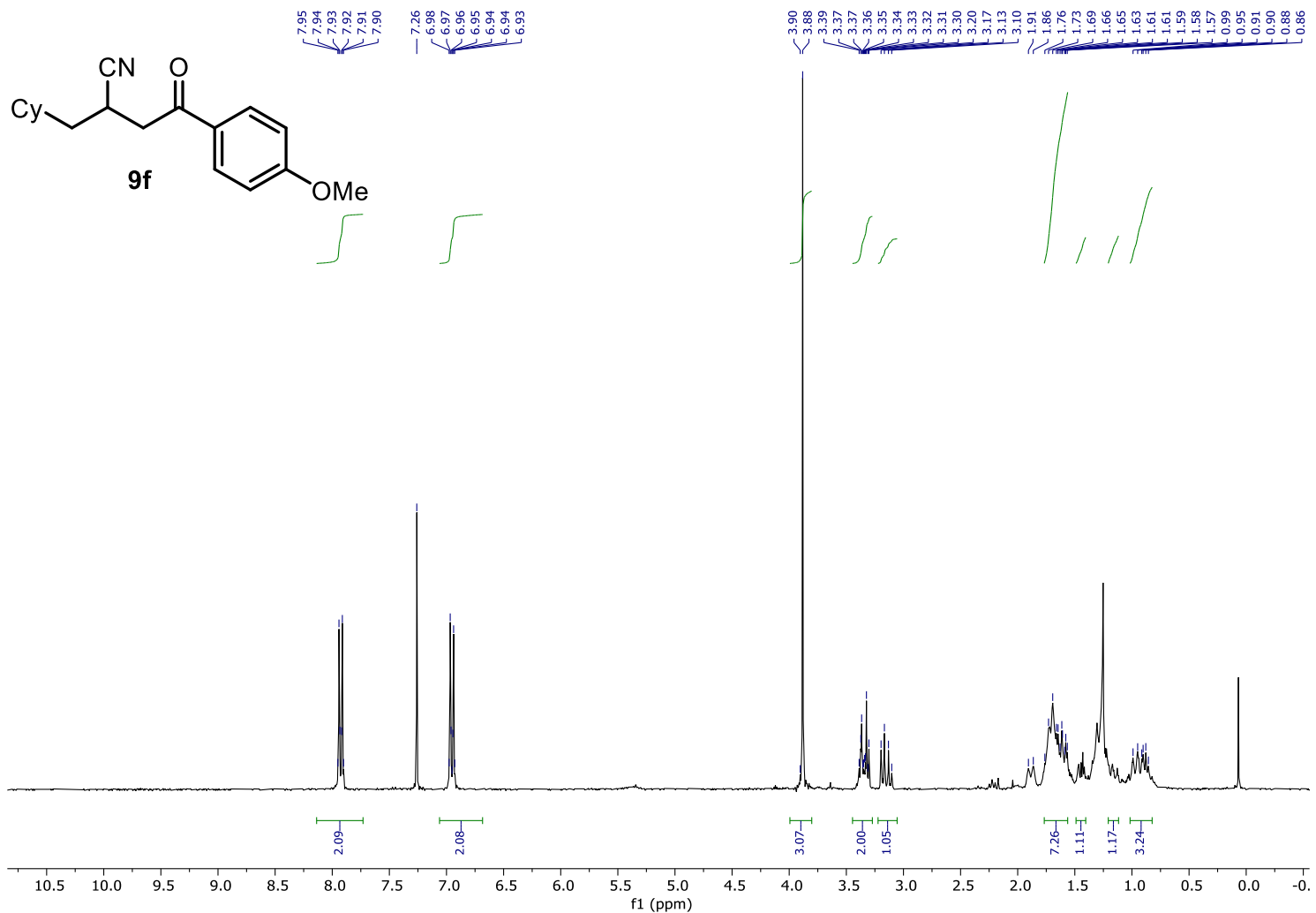

${ }^{13} \mathrm{C}$ NMR $\left(75 \mathrm{MHz}, \mathrm{CDCl}_{3}\right)$

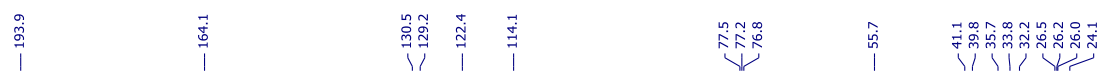

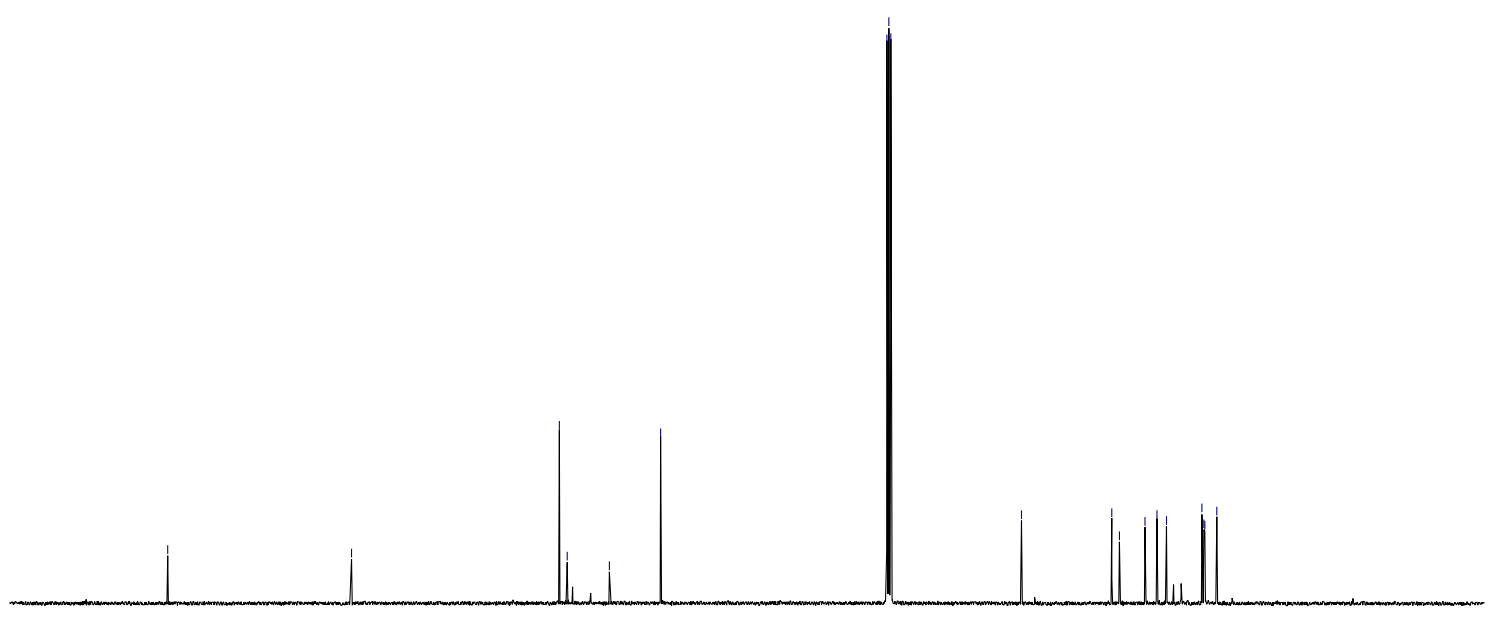

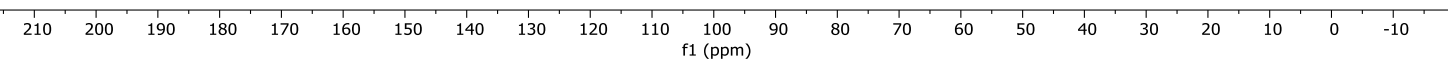


${ }^{1} \mathrm{H}$ NMR (400 MHz, $\mathrm{CDCl}_{3}$ )

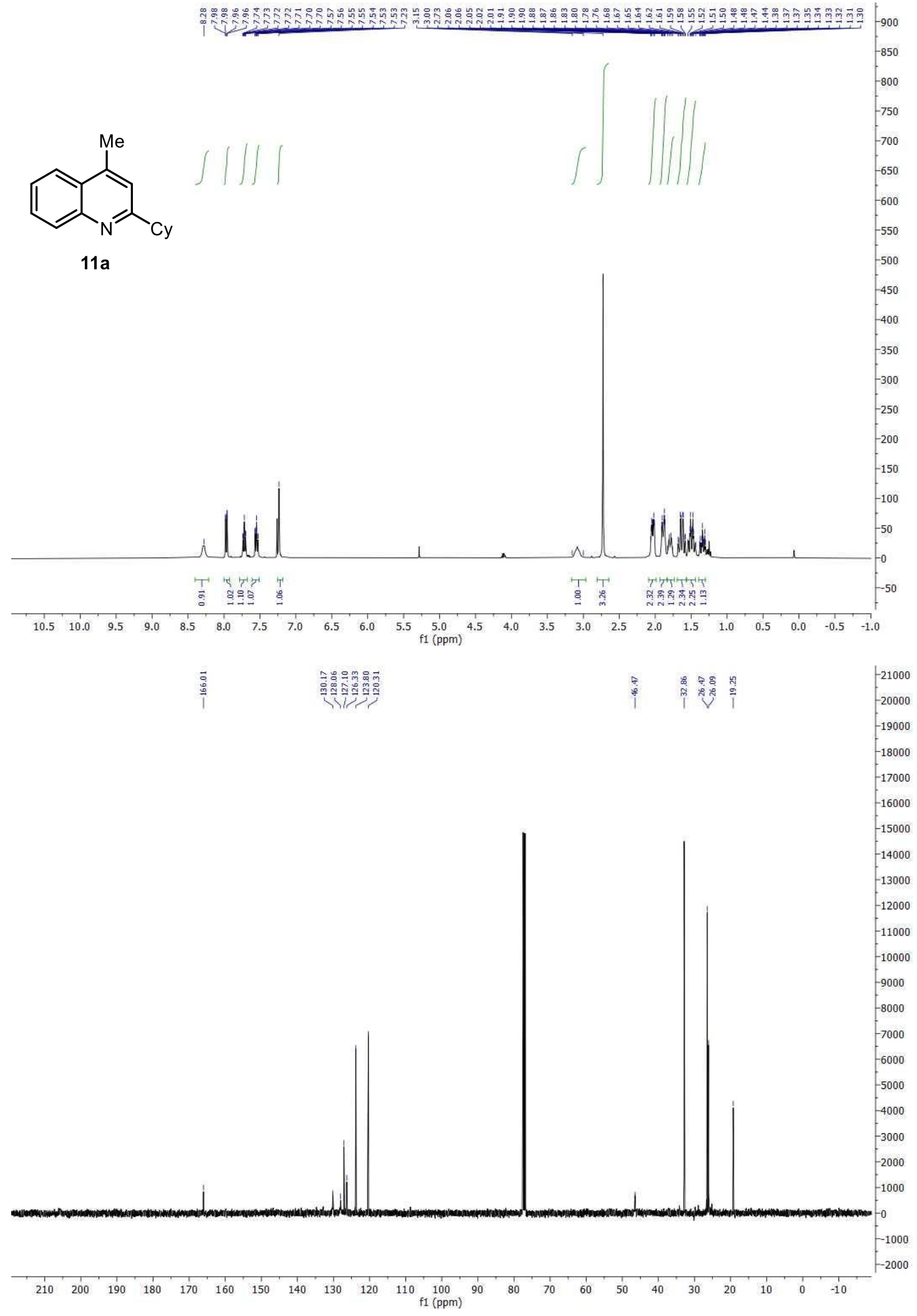


${ }^{1} \mathrm{H}$ NMR (400 MHz, $\mathrm{CDCl}_{3}$ )

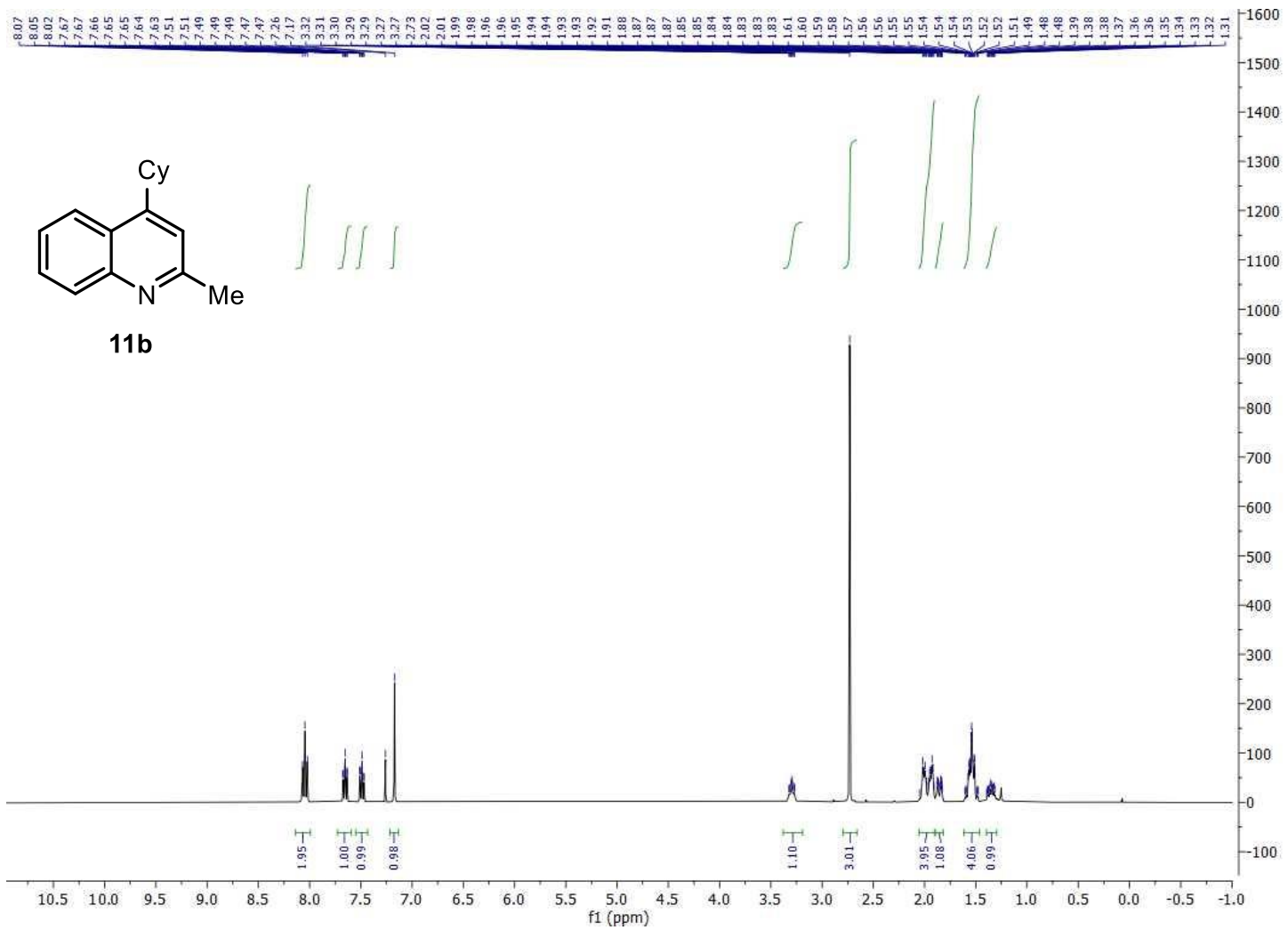

${ }^{1} \mathrm{H}$ NMR (400 MHz, $\mathrm{CDCl}_{3}$ )

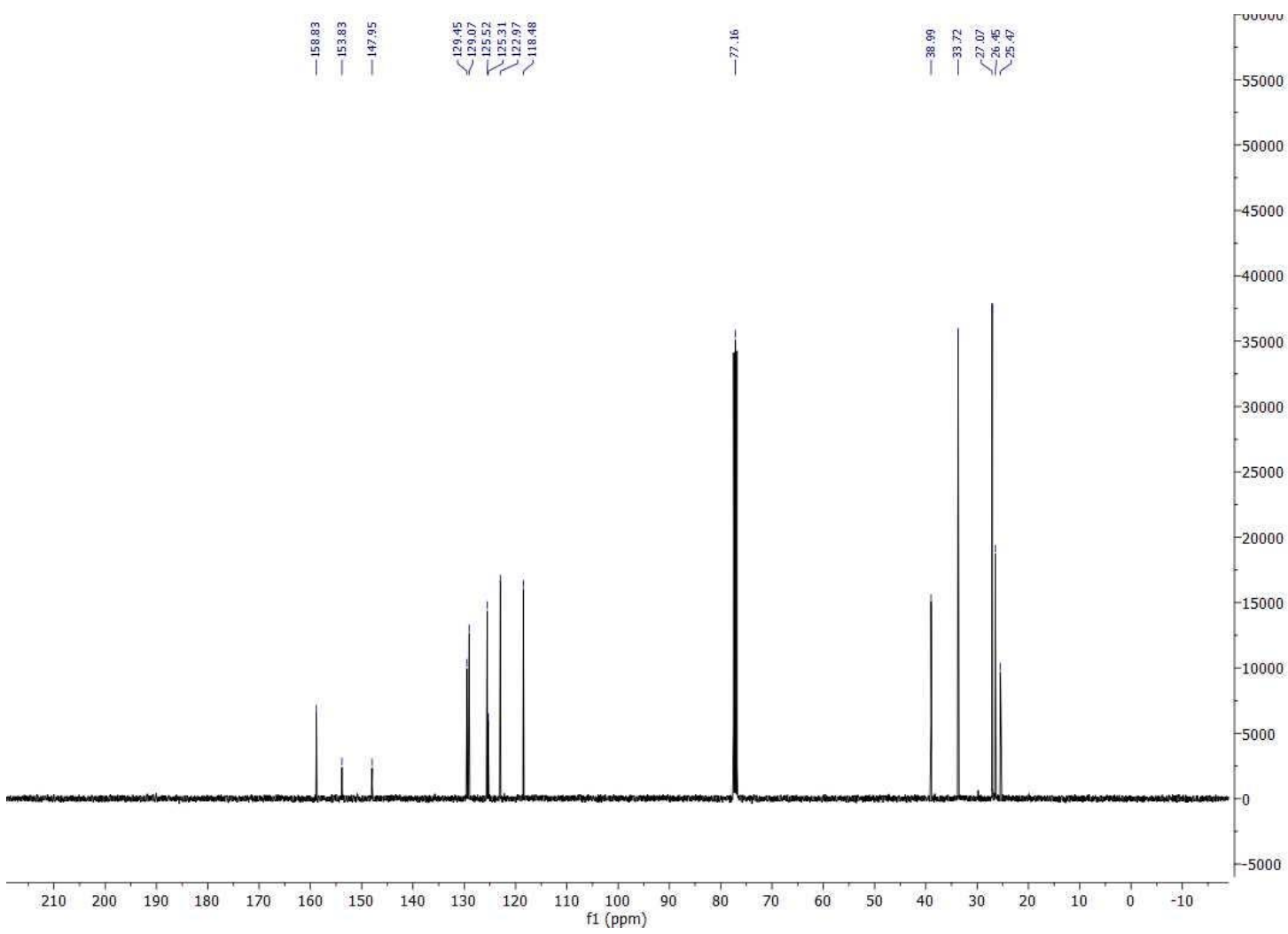


${ }^{1} \mathrm{H}$ NMR (400 MHz, $\mathrm{CDCl}_{3}$ )

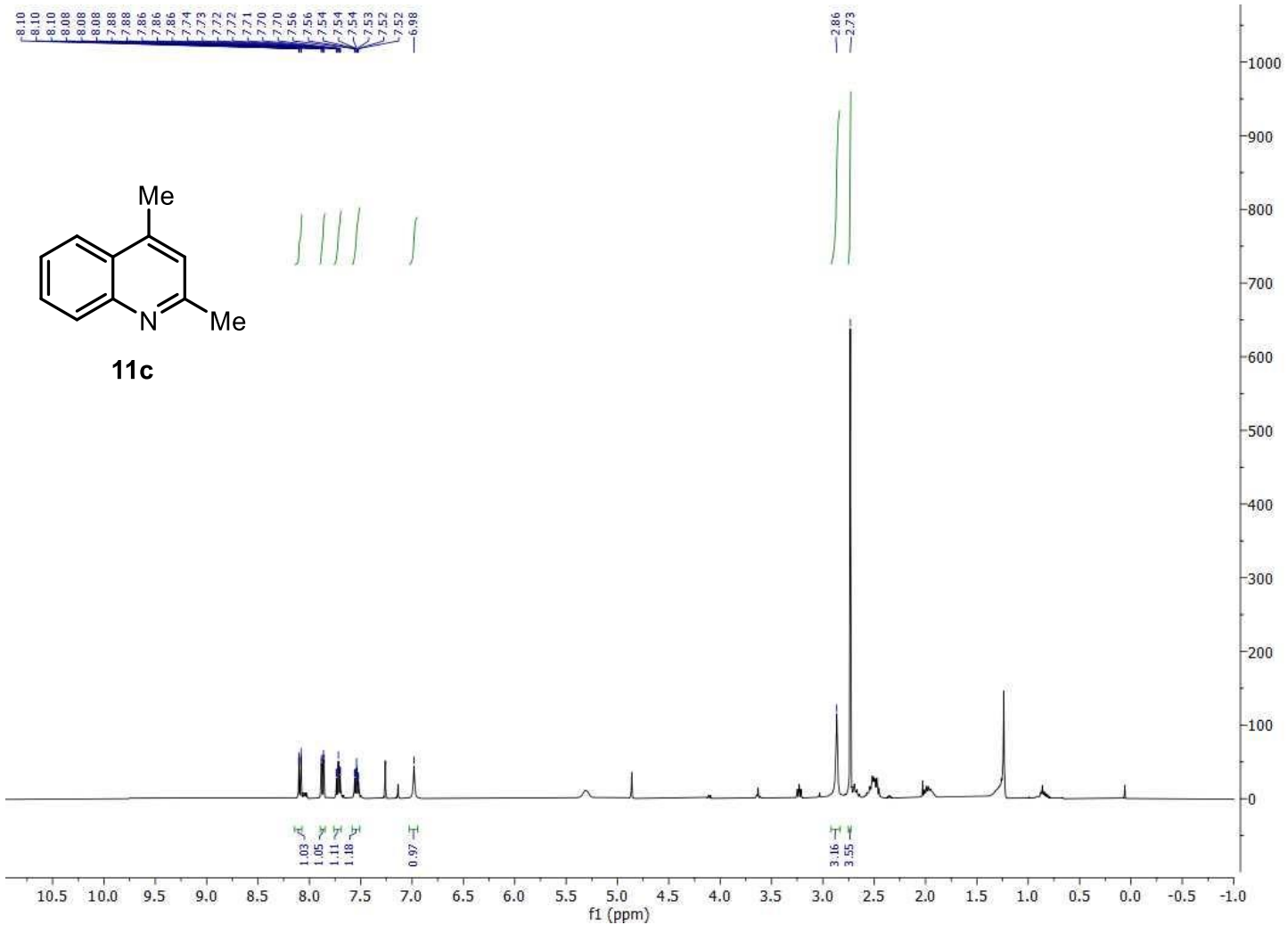

${ }^{13} \mathrm{C}$ NMR $\left(101 \mathrm{MHz}, \mathrm{CDCl}_{3}\right)$

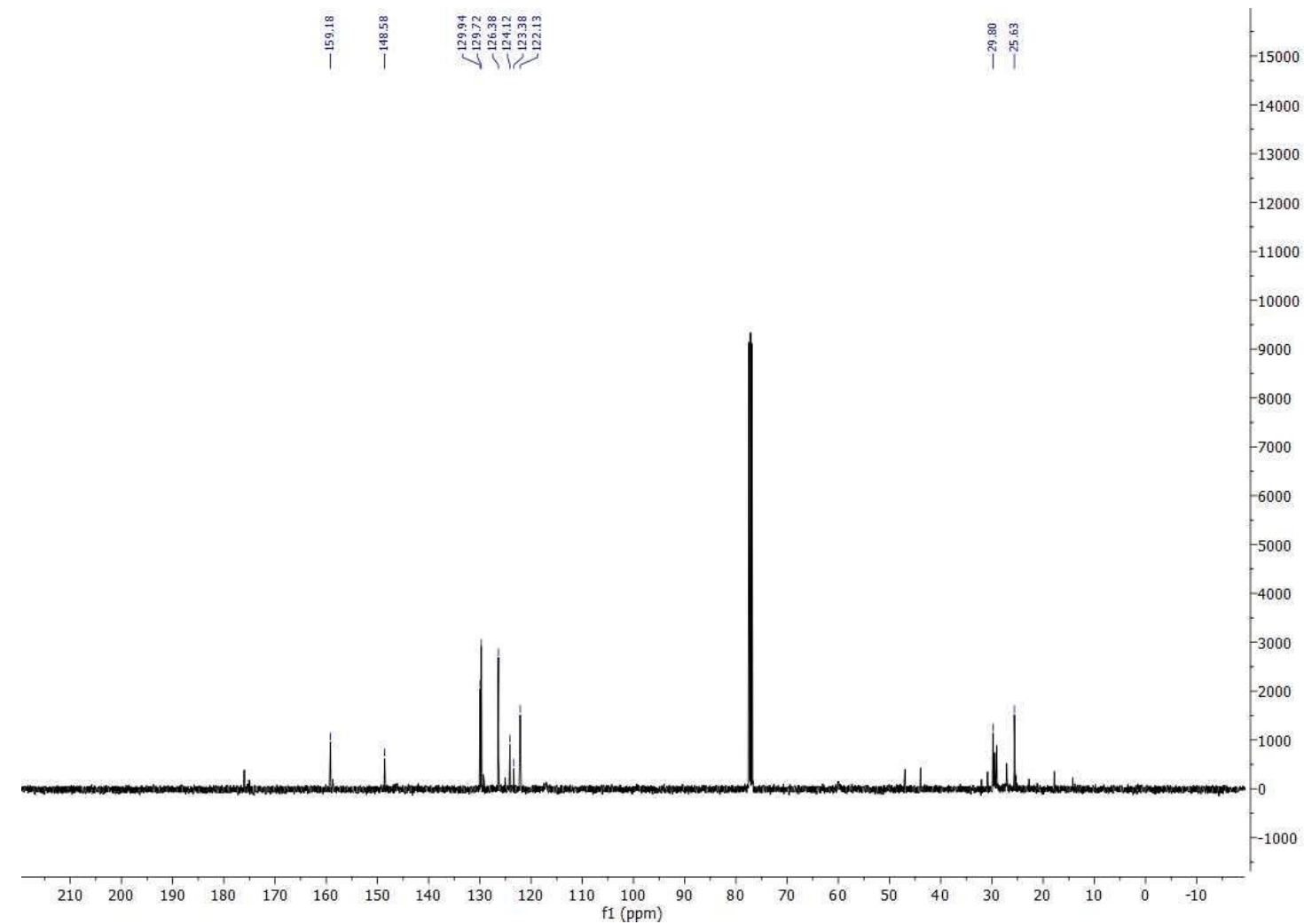


${ }^{1} \mathrm{H}$ NMR (400 MHz, $\left.\mathrm{CDCl}_{3}\right)$

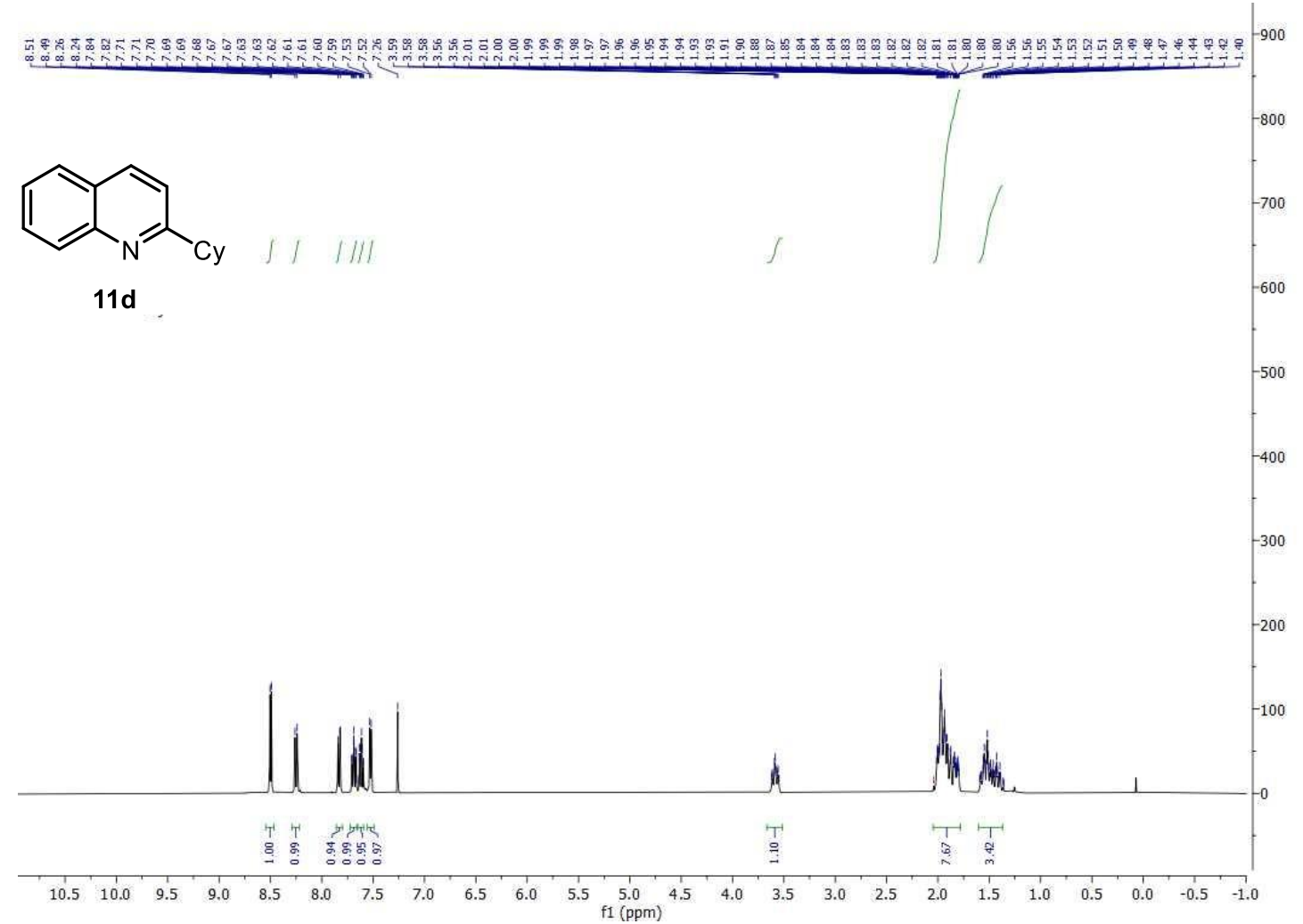

${ }^{13} \mathrm{C}$ NMR (101 MHz, $\left.\mathrm{CDCl}_{3}\right)$

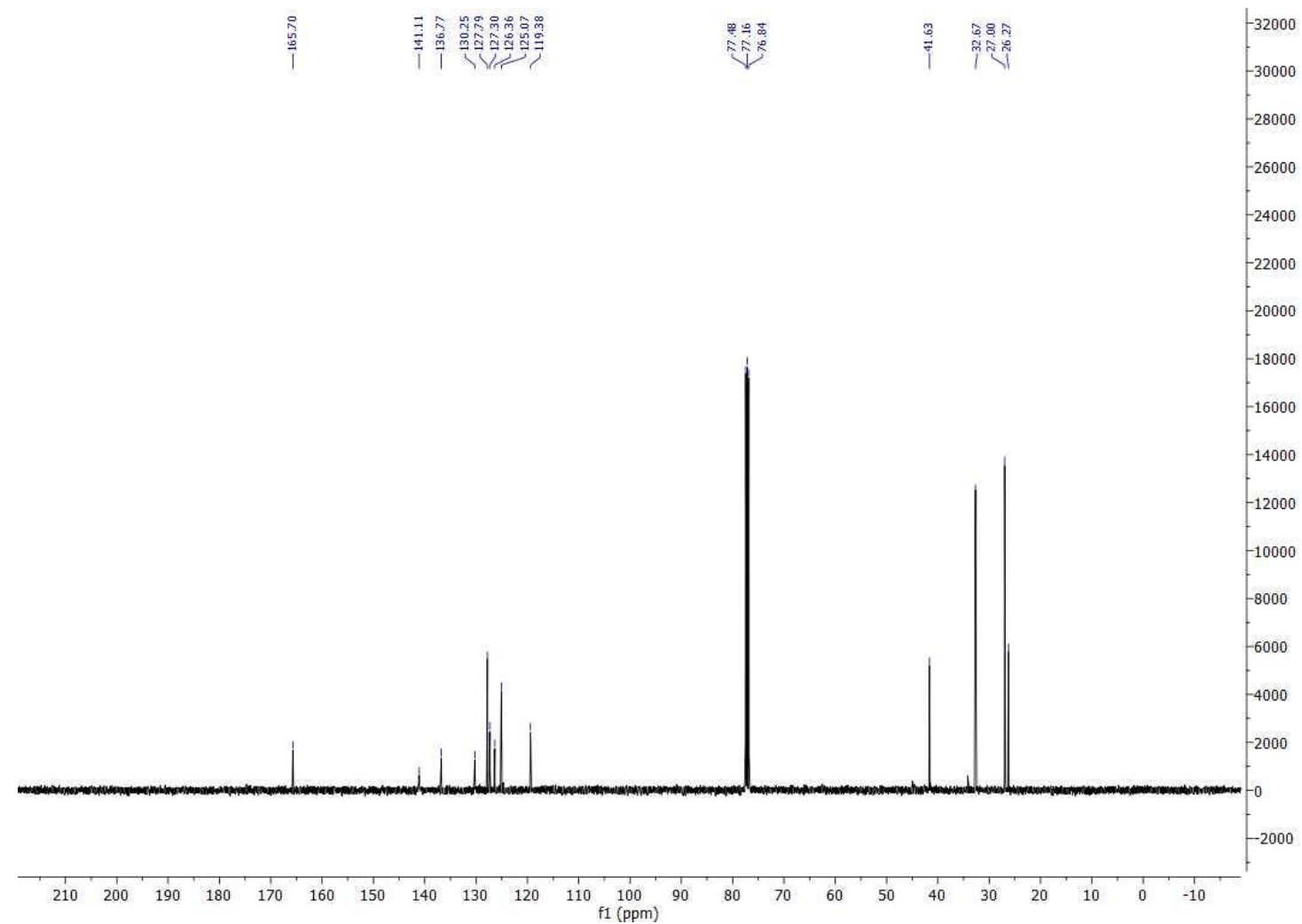


${ }^{1} \mathrm{H}$ NMR (400 MHz, $\left.\mathrm{CDCl}_{3}\right)$

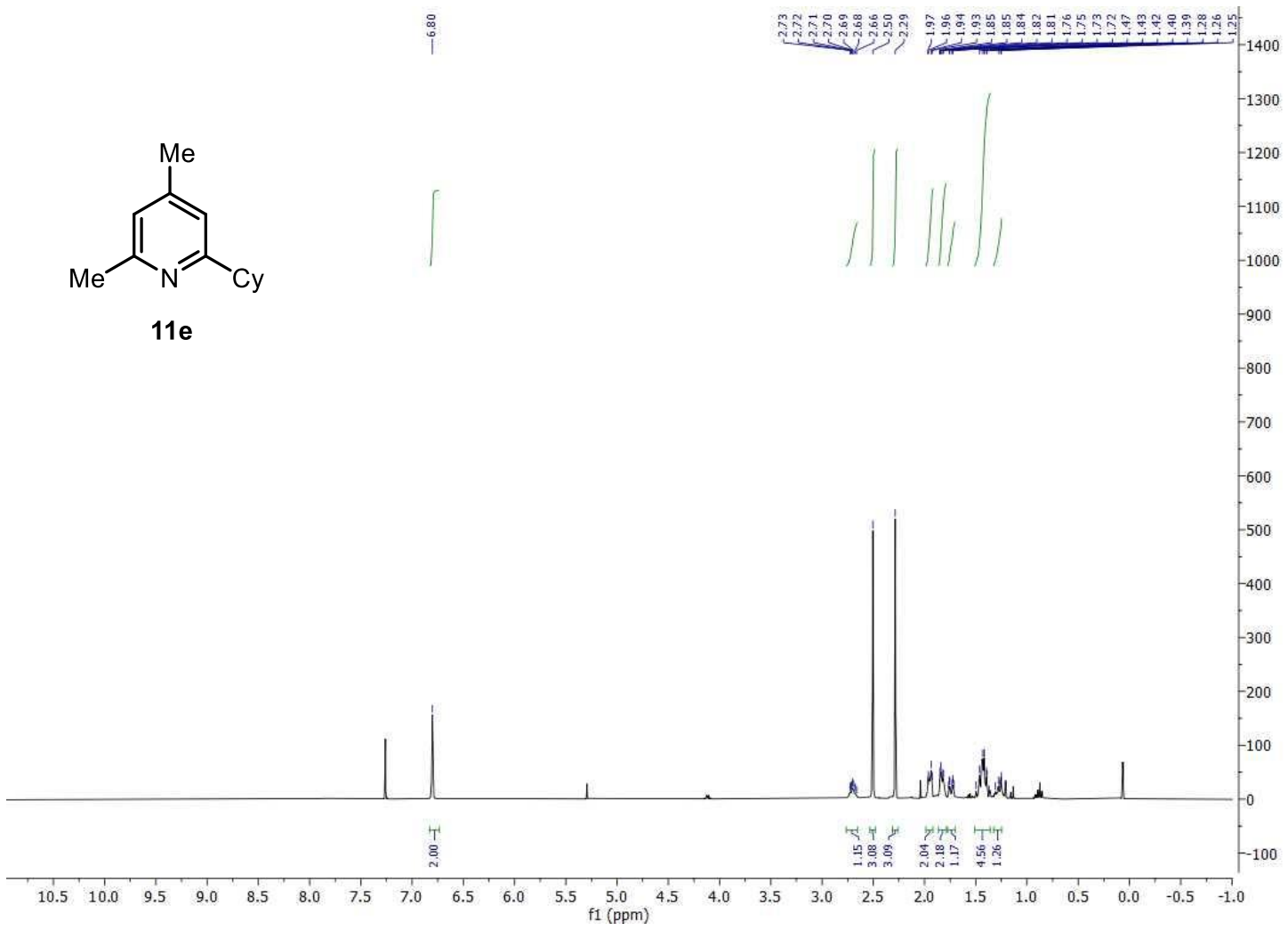

${ }^{13} \mathrm{C}$ NMR $\left(101 \mathrm{MHz}, \mathrm{CDCl}_{3}\right)$

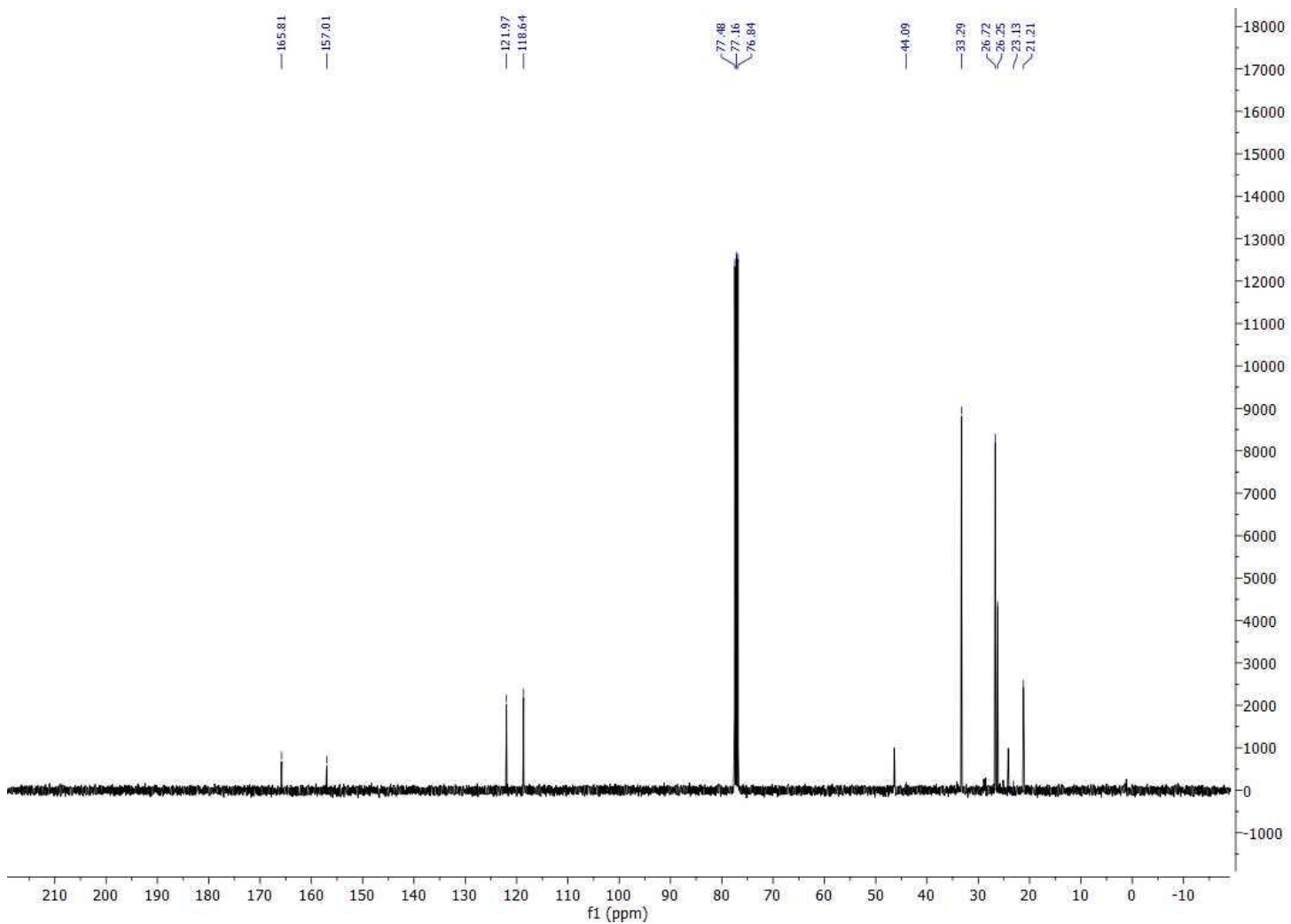


${ }^{1} \mathrm{H}$ NMR (400 MHz, $\mathrm{CDCl}_{3}$ )

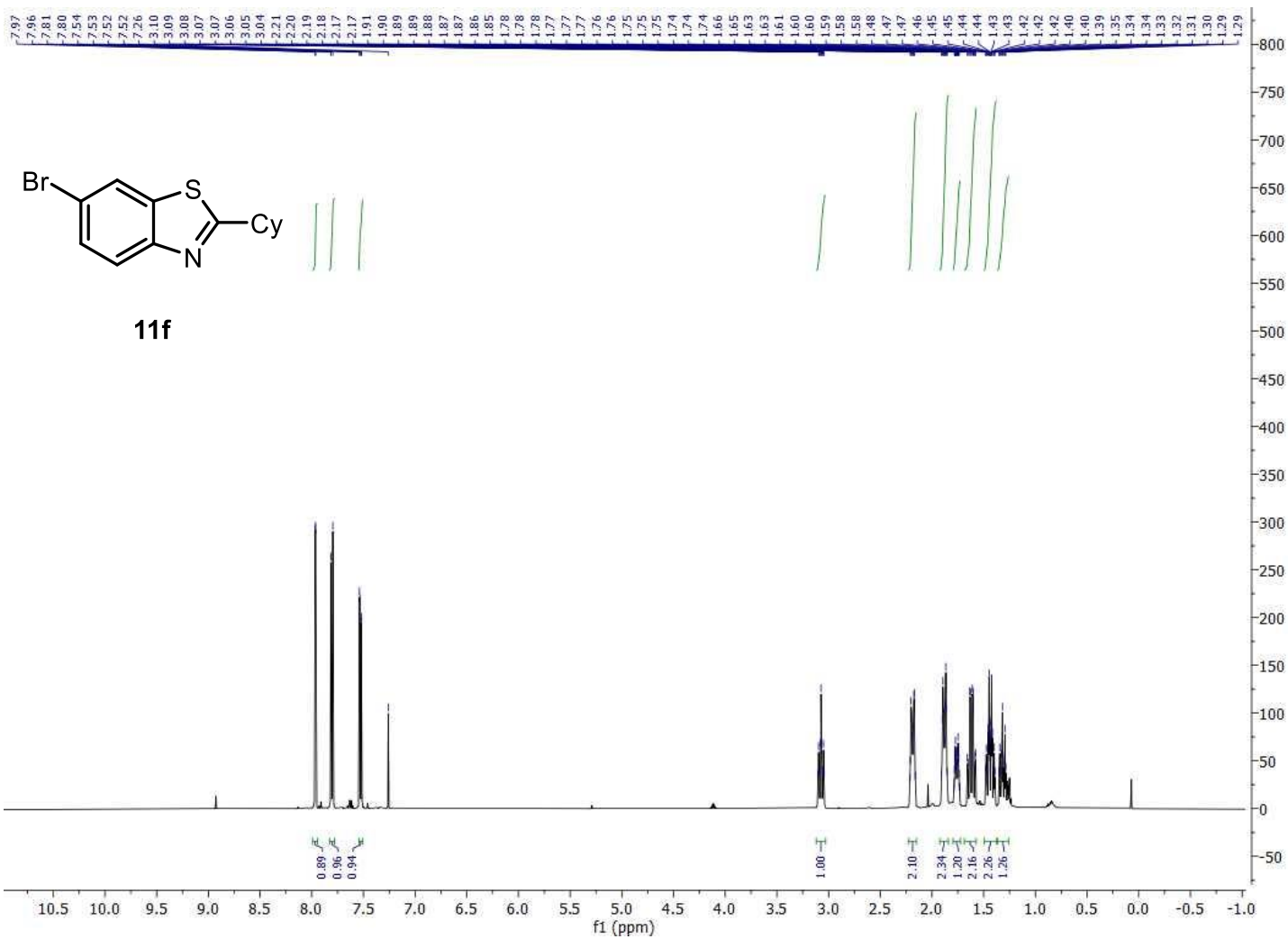

${ }^{13} \mathrm{C}$ NMR (101 MHz, $\left.\mathrm{CDCl}_{3}\right)$

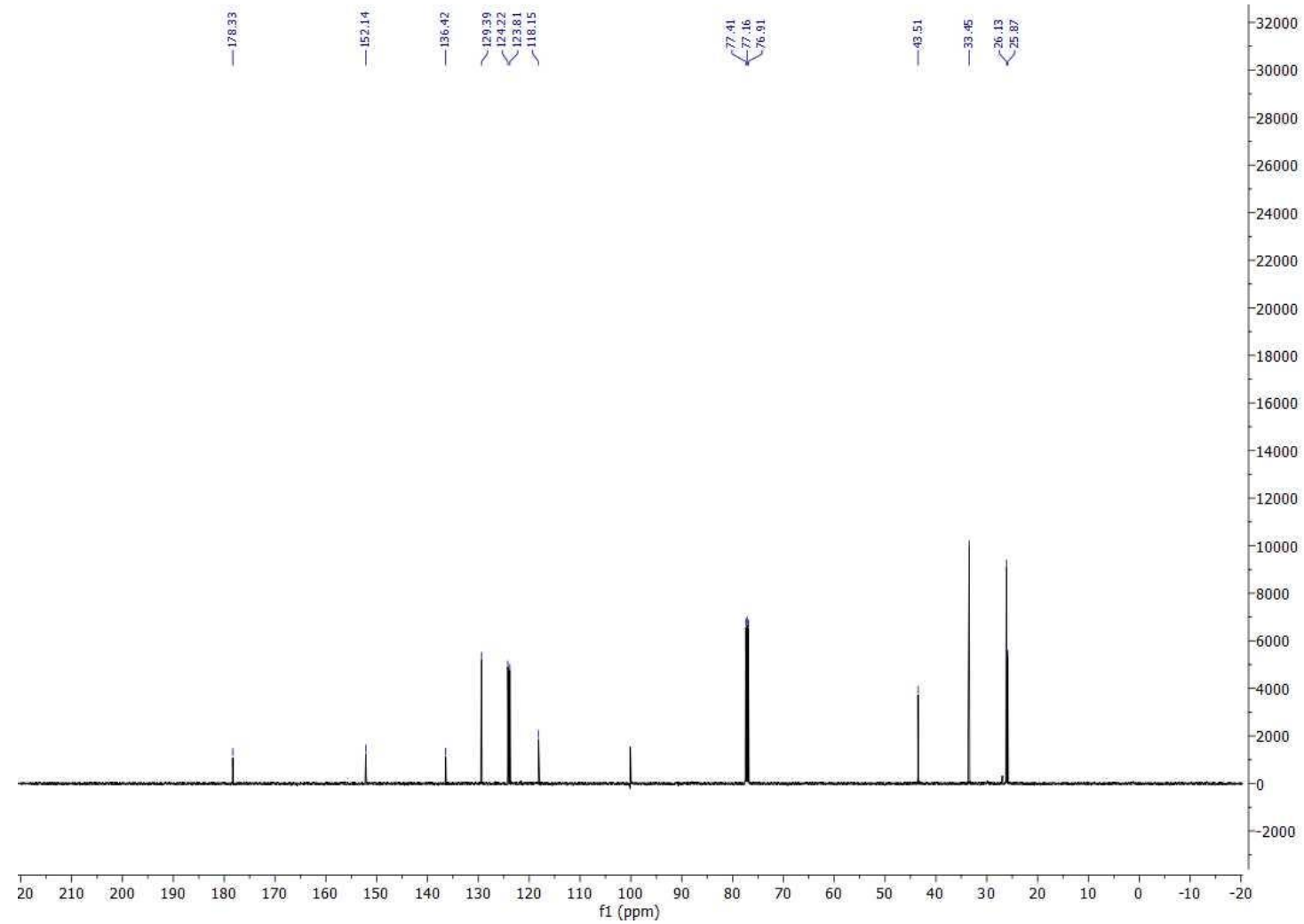


${ }^{1} \mathrm{H}$ NMR (400 MHz, $\mathrm{CDCl}_{3}$ )

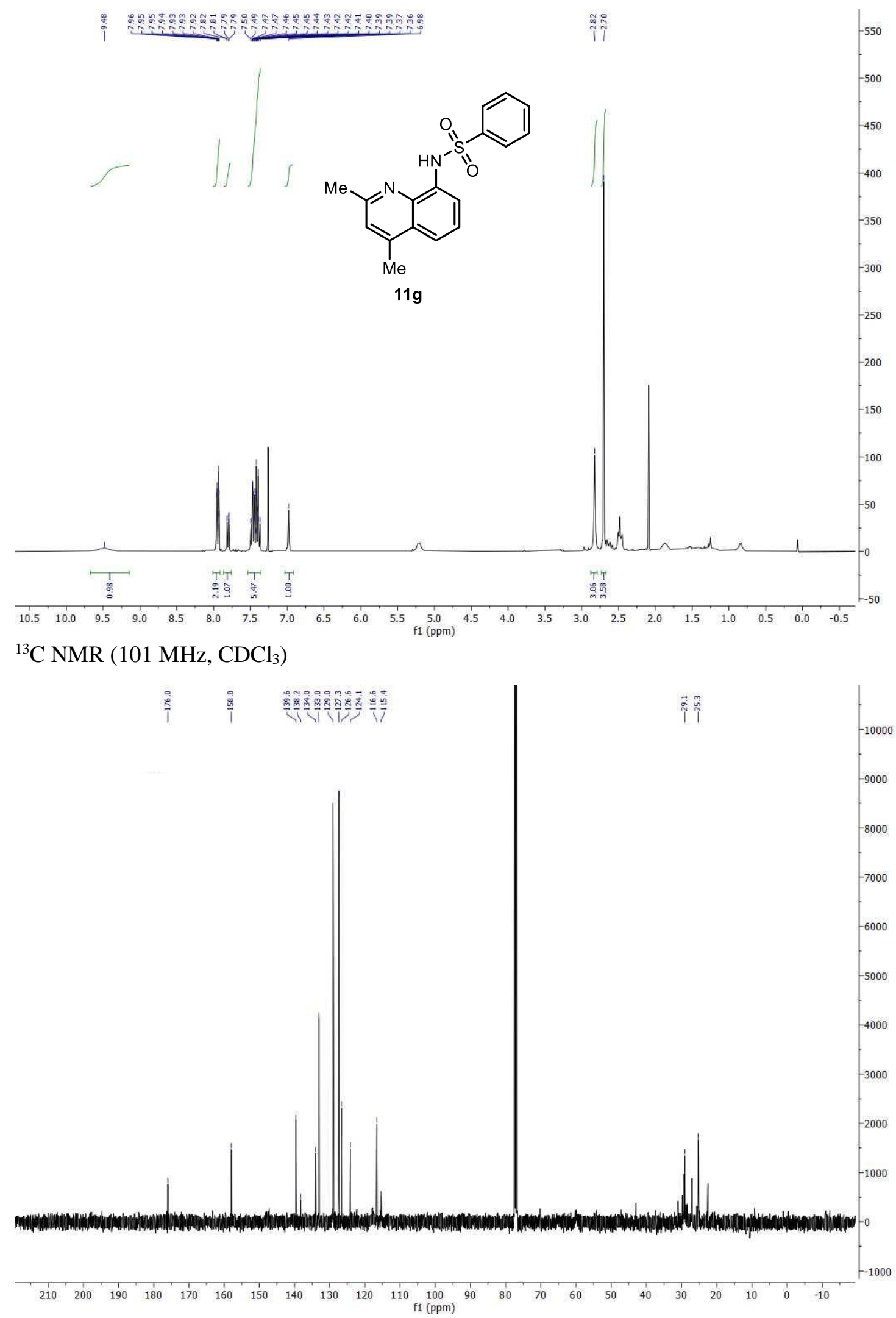


${ }^{1} \mathrm{H}$ NMR (400 MHz, $\left.\mathrm{CDCl}_{3}\right)$

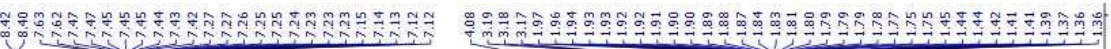<smiles>C#CCOc1ccc2c(O)c(C(=O)OC)nc(Cl)c2c1</smiles>

11h
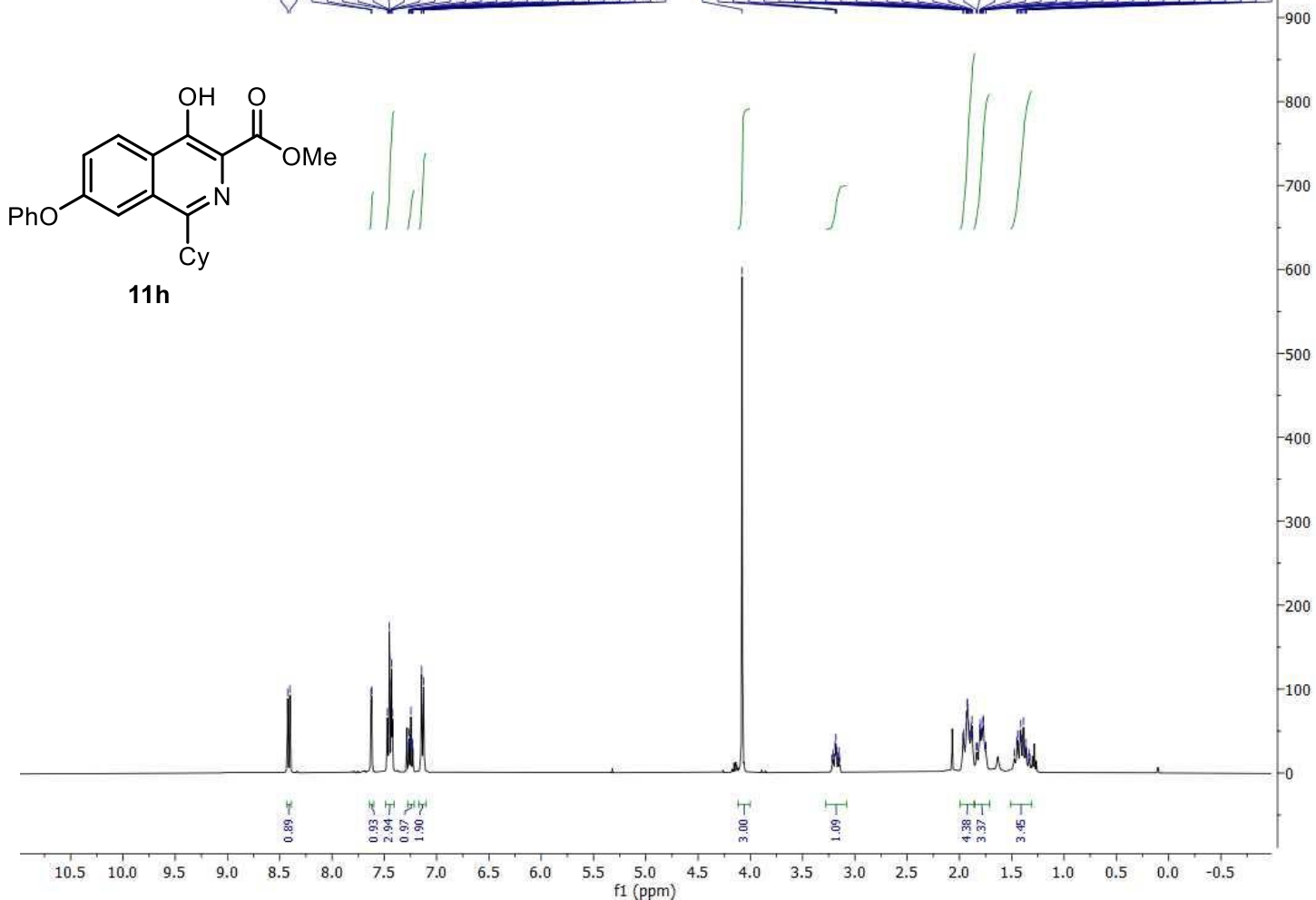

${ }^{13} \mathrm{C}$ NMR $\left(101 \mathrm{MHz}, \mathrm{CDCl}_{3}\right)$

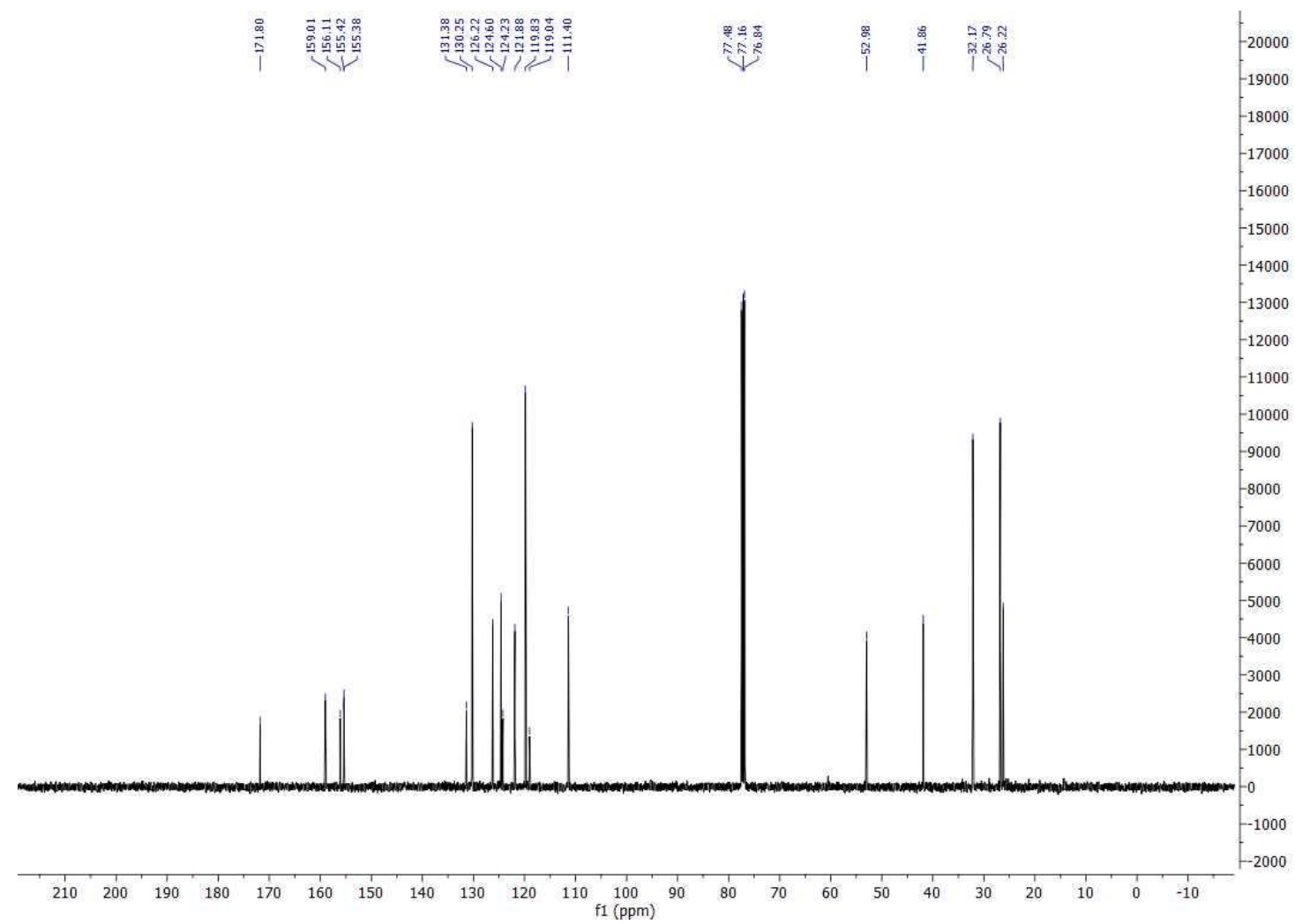


${ }^{1} \mathrm{H}$ NMR (400 MHz, $\left.\mathrm{CDCl}_{3}\right)$

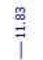

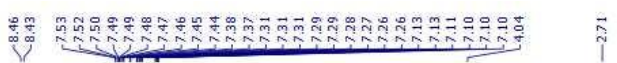

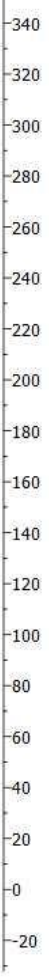

$\begin{array}{llllllllllllllllllllllllllllllllllll}12.5 & 12.0 & 11.5 & 11.0 & 10.5 & 10.0 & 9.5 & 9.0 & 8.5 & 8.0 & 7.5 & 7.0 & 6.5 & 6.0 & 5.5 & 5.0 & 4.5 & 4.0 & 3.5 & 3.0 & 2.5 & 2.0 & 1.5 & 1.0 & 0.5 & 0.0 & -0.5\end{array}$

${ }^{13} \mathrm{C}$ NMR $\left(101 \mathrm{MHz}, \mathrm{CDCl}_{3}\right)$

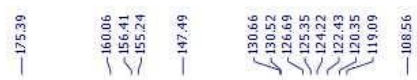

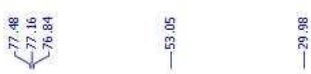

14000

13000

$-2000$

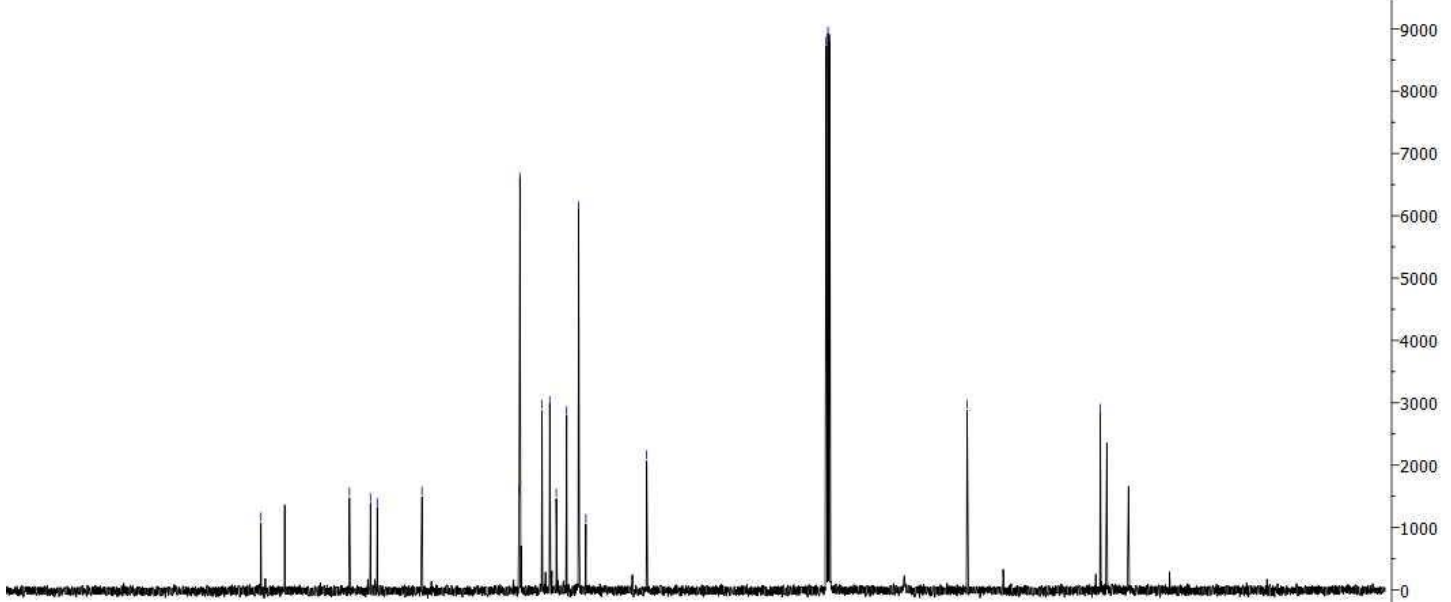

$\begin{array}{lllllllllllllllllllllllllllllll}210 & 200 & 190 & 180 & 170 & 160 & 150 & 140 & 130 & 120 & 110 & 100 & 90 & 80 & 70 & 60 & 50 & 40 & 30 & 20 & 10 & 0 & -10 & 10 & 1\end{array}$ 
${ }^{1} \mathrm{H}$ NMR (400 MHz, $\mathrm{CDCl}_{3}$ )

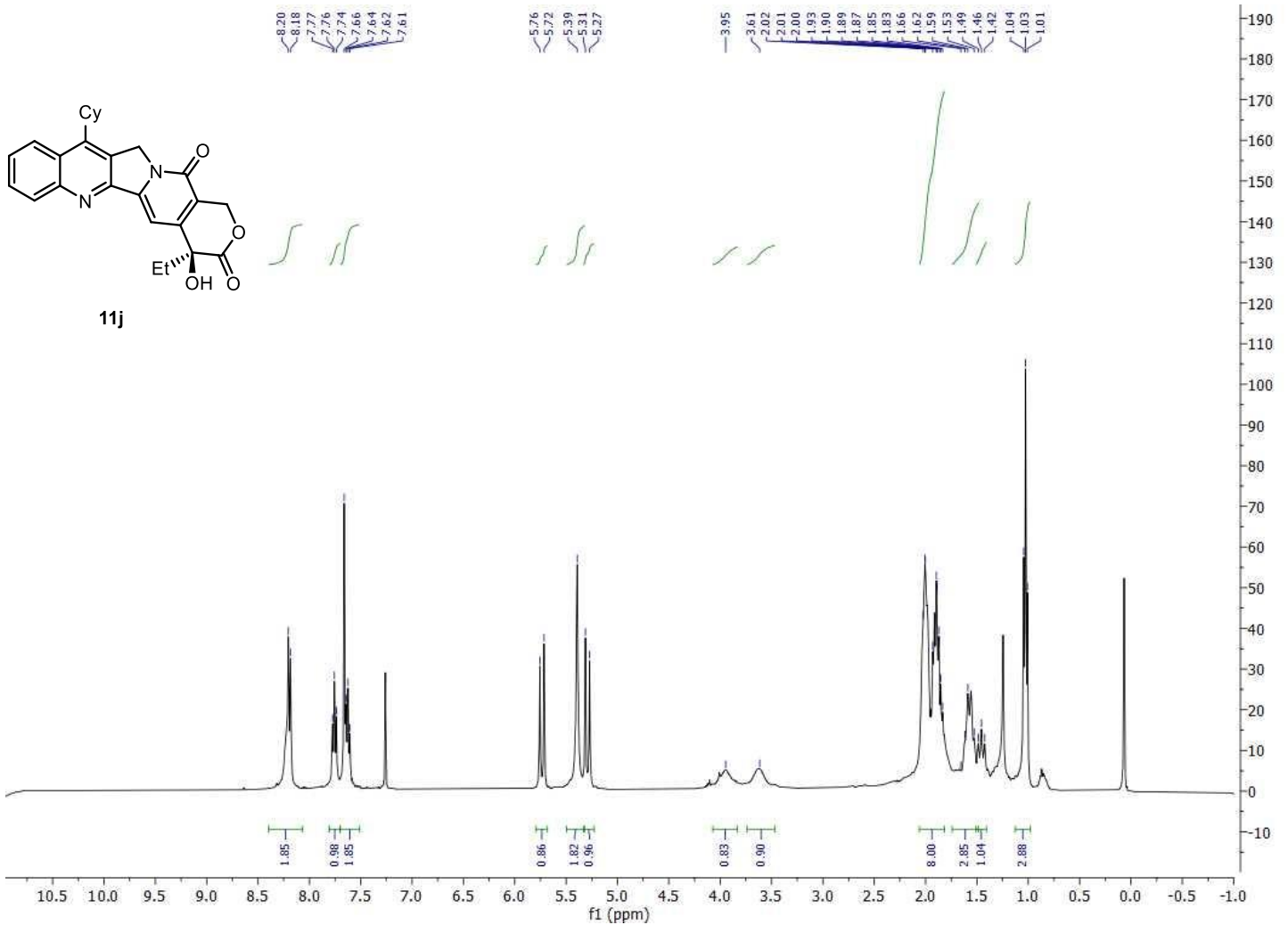

${ }^{13} \mathrm{C}$ NMR (101 MHz, $\left.\mathrm{CDCl}_{3}\right)$

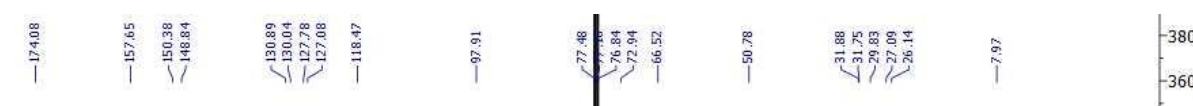

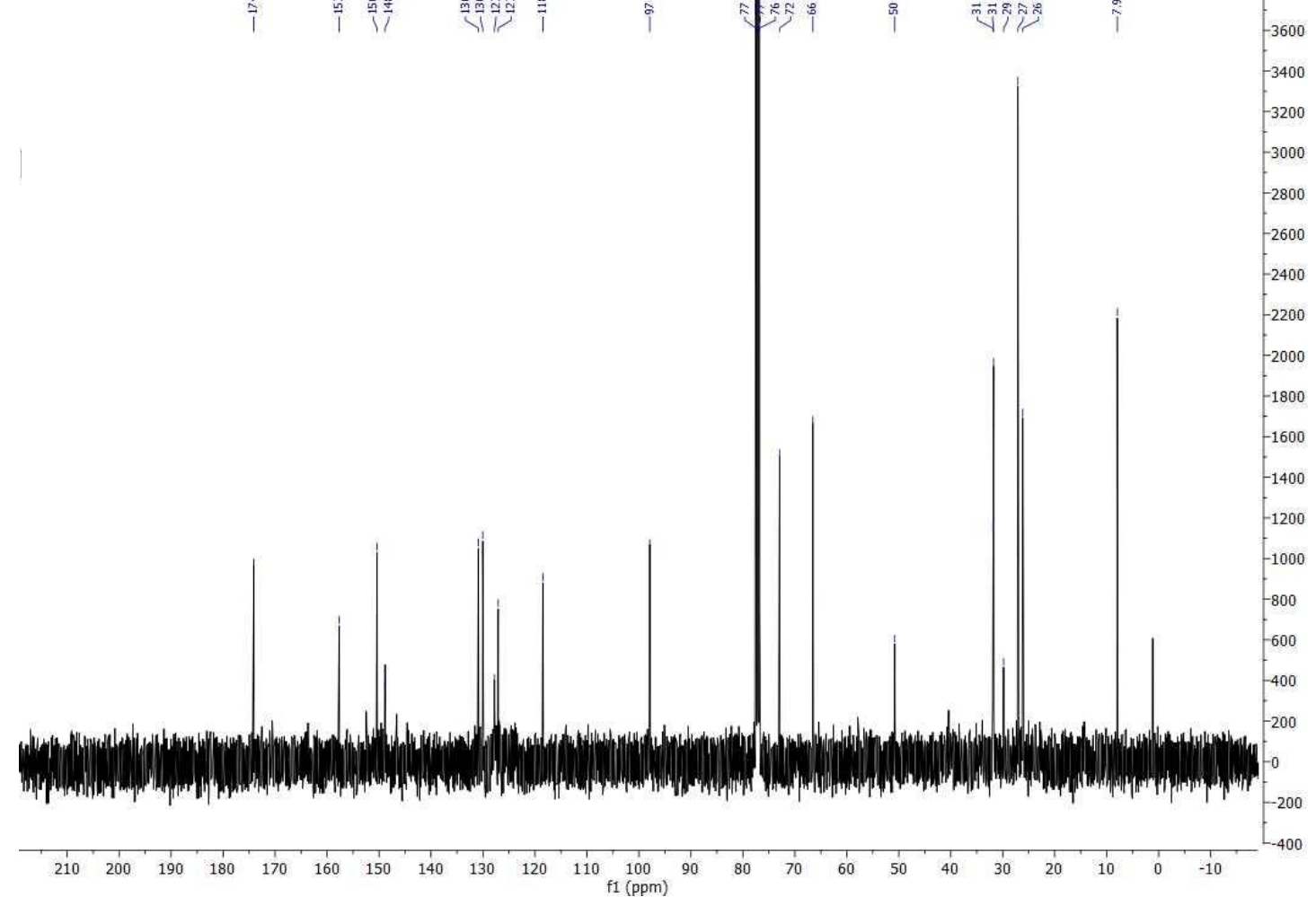


${ }^{1} \mathrm{H}$ NMR (400 MHz, Acetone- $d_{6}$ )

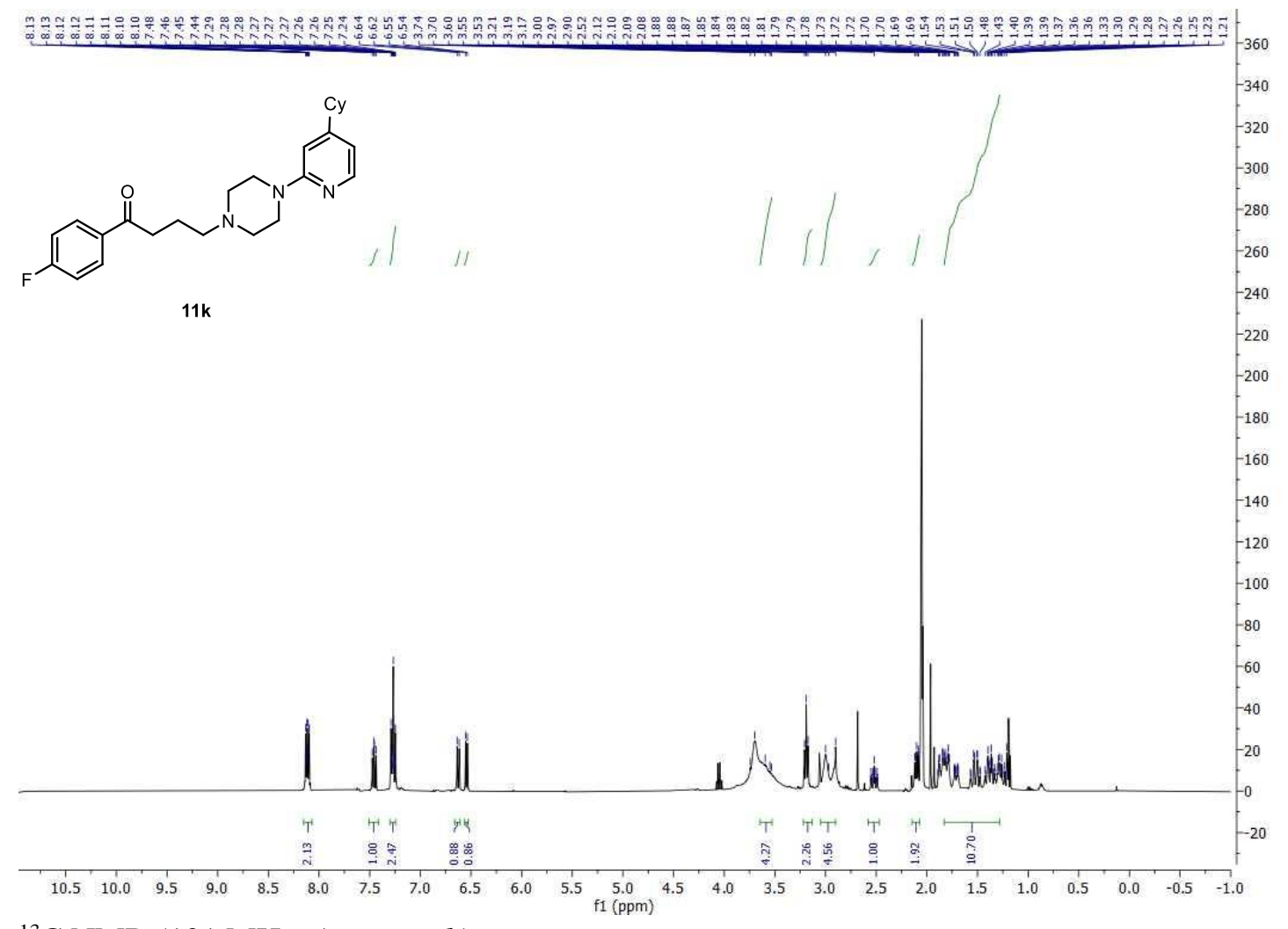

${ }^{13} \mathrm{C}$ NMR (101 MHz, Acetone- $d_{6}$ )

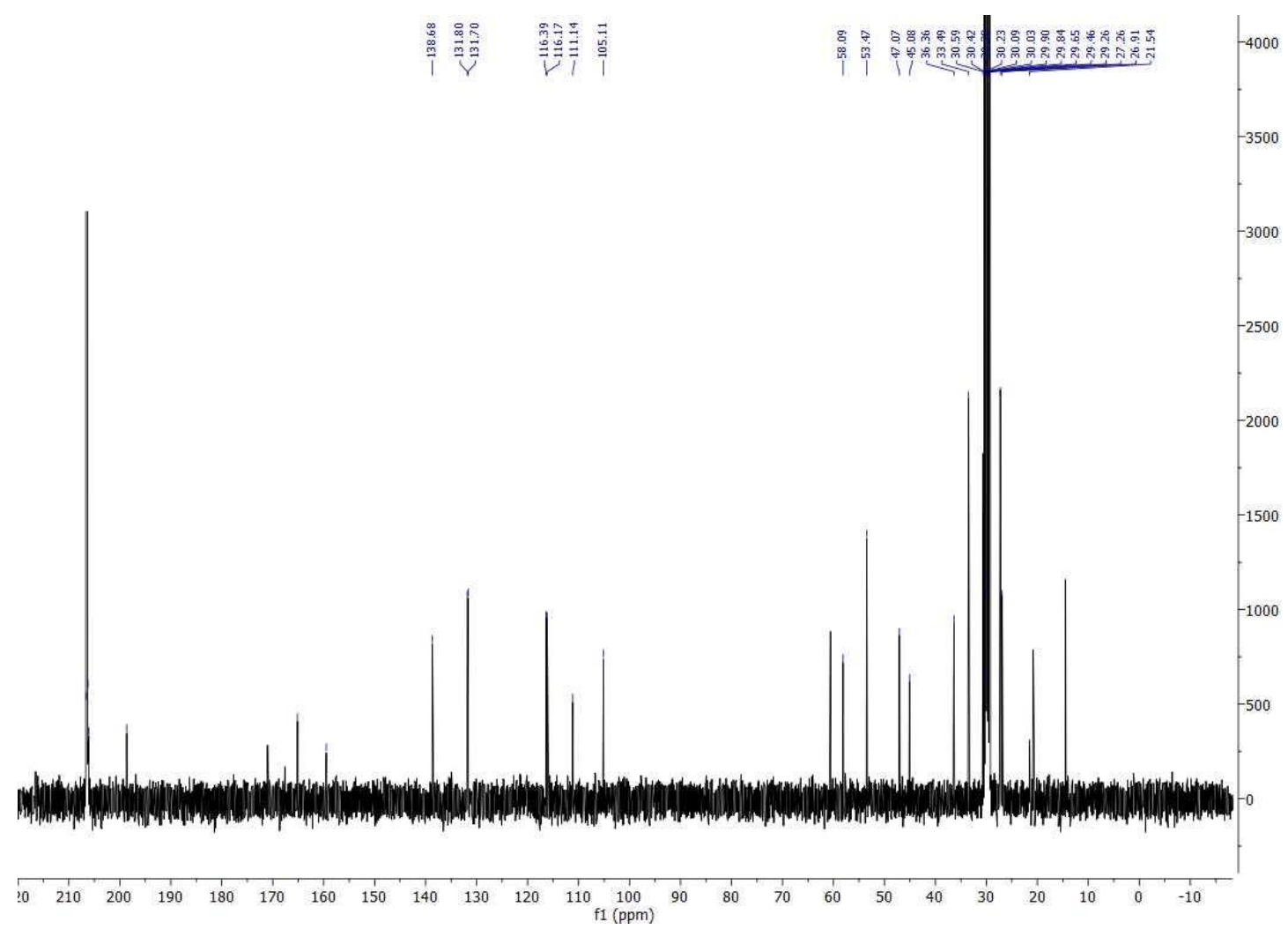


${ }^{19} \mathrm{~F}$ NMR (376 MHz, Acetone- $\left.d_{6}\right)$

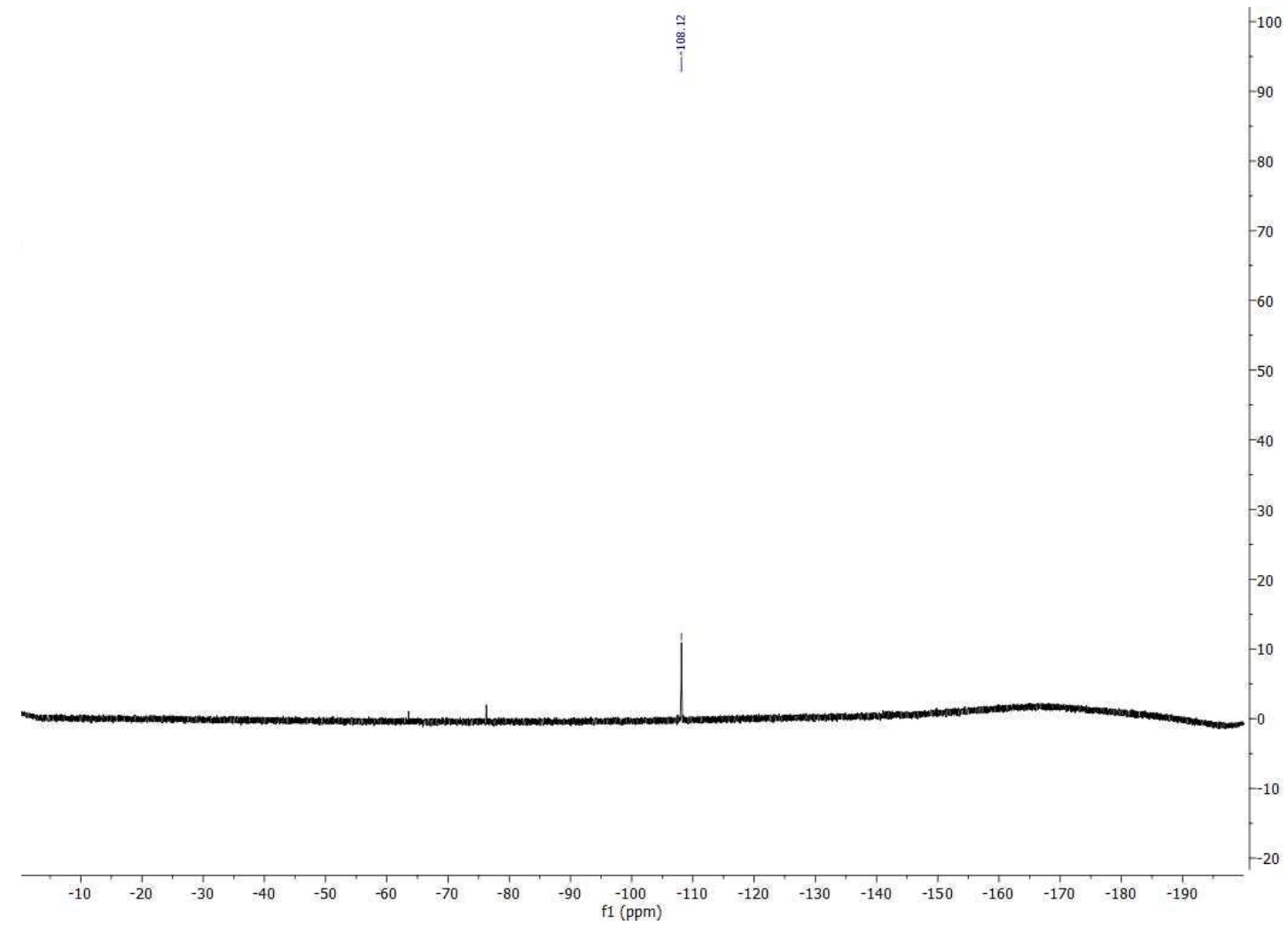


${ }^{1} \mathrm{H}$ NMR (400 MHz, $\mathrm{CDCl}_{3}$ )

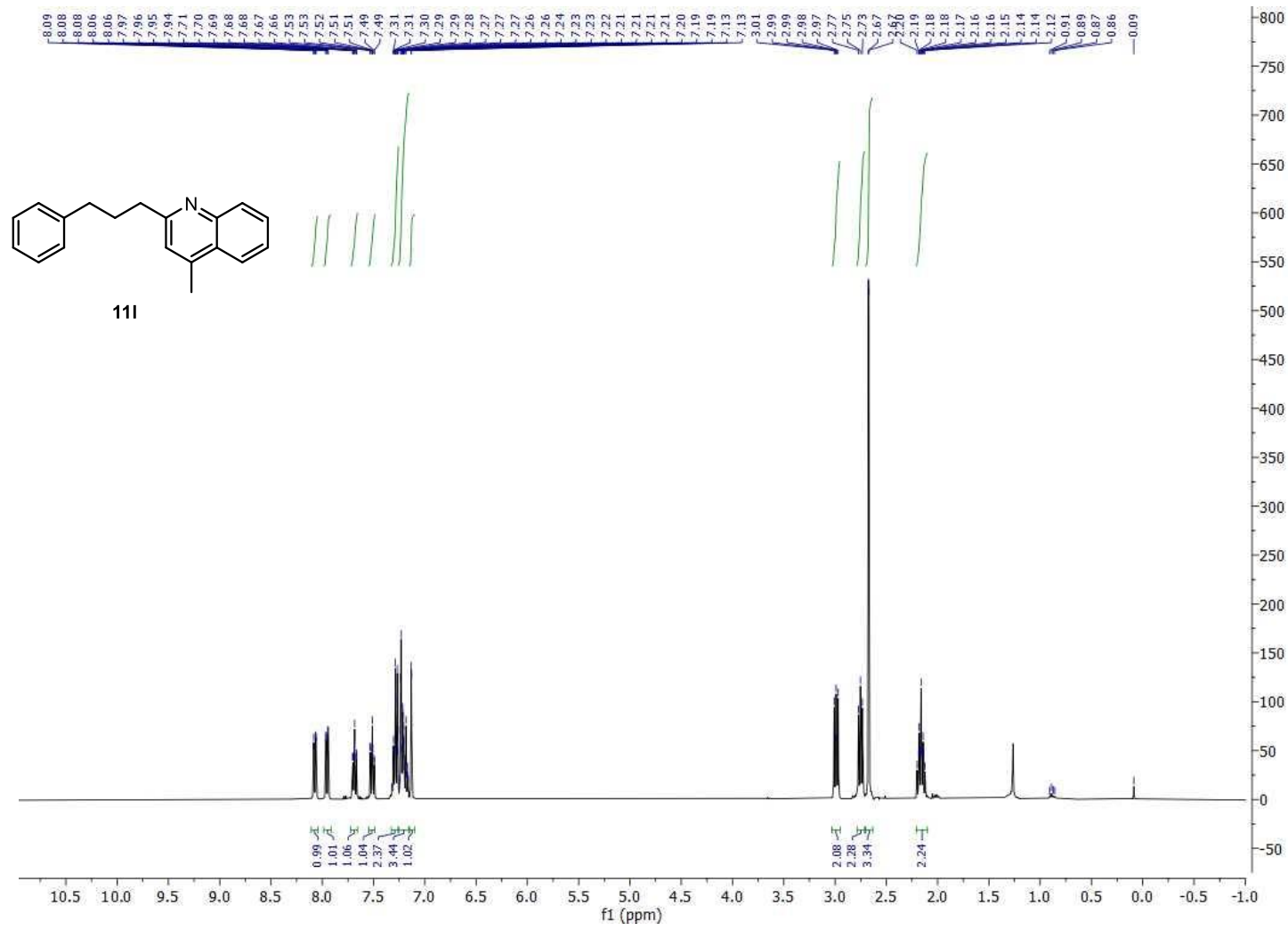

${ }^{13} \mathrm{C}$ NMR (101 MHz, $\left.\mathrm{CDCl}_{3}\right)$

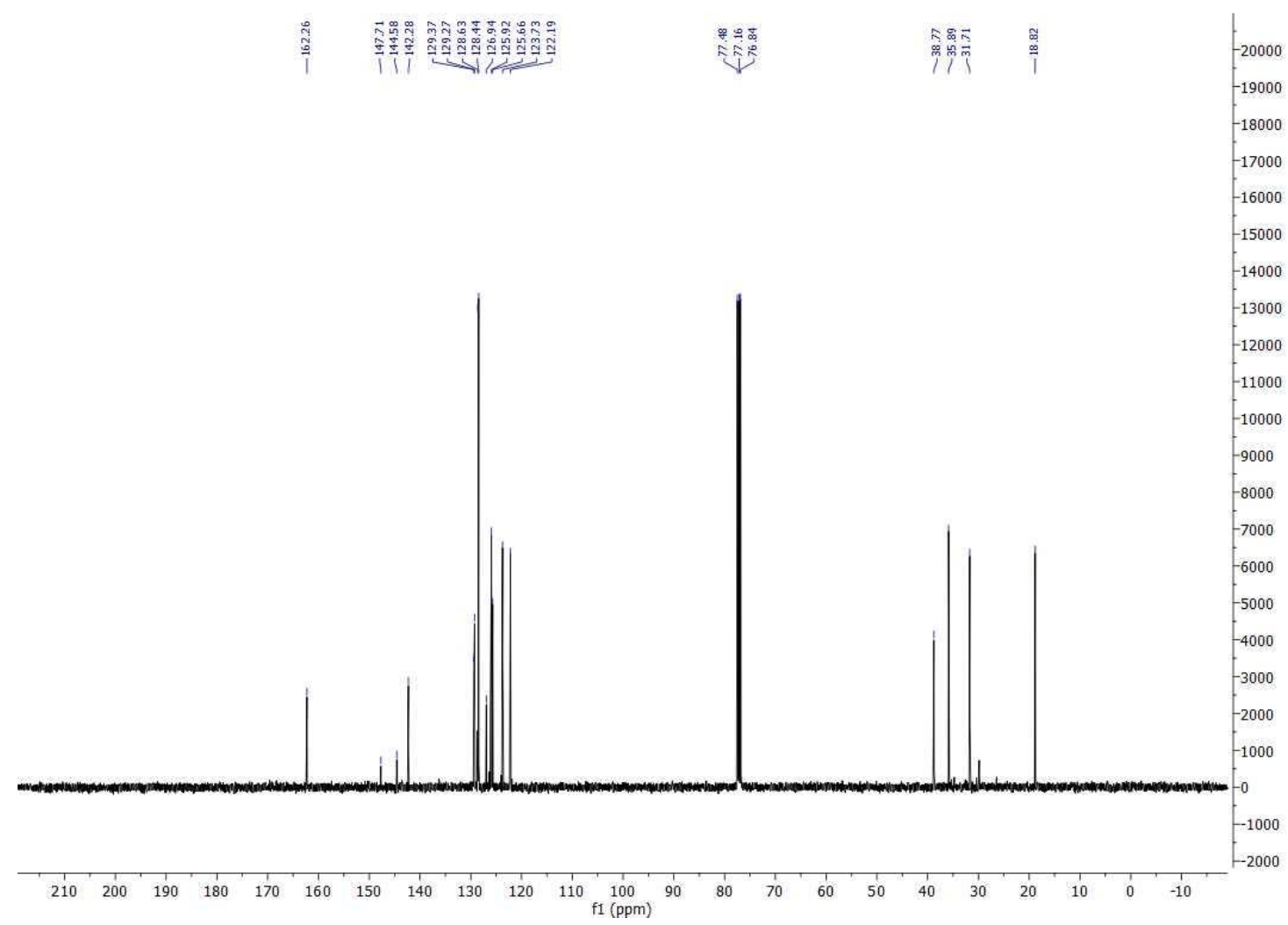


${ }^{1} \mathrm{H}$ NMR (400 MHz, $\mathrm{CDCl}_{3}$ )

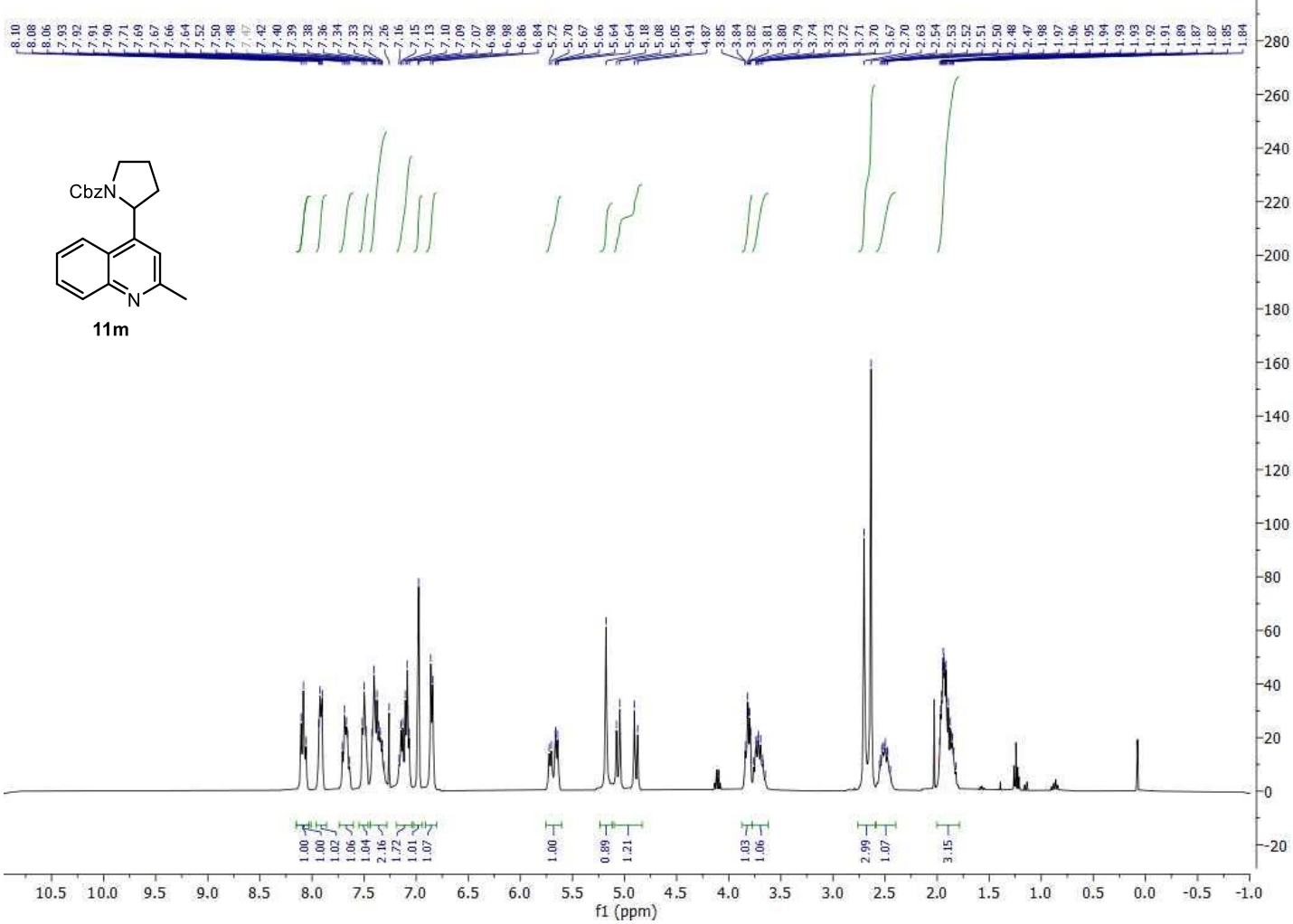

${ }^{13} \mathrm{C}$ NMR $\left(101 \mathrm{MHz}, \mathrm{CDCl}_{3}\right)$

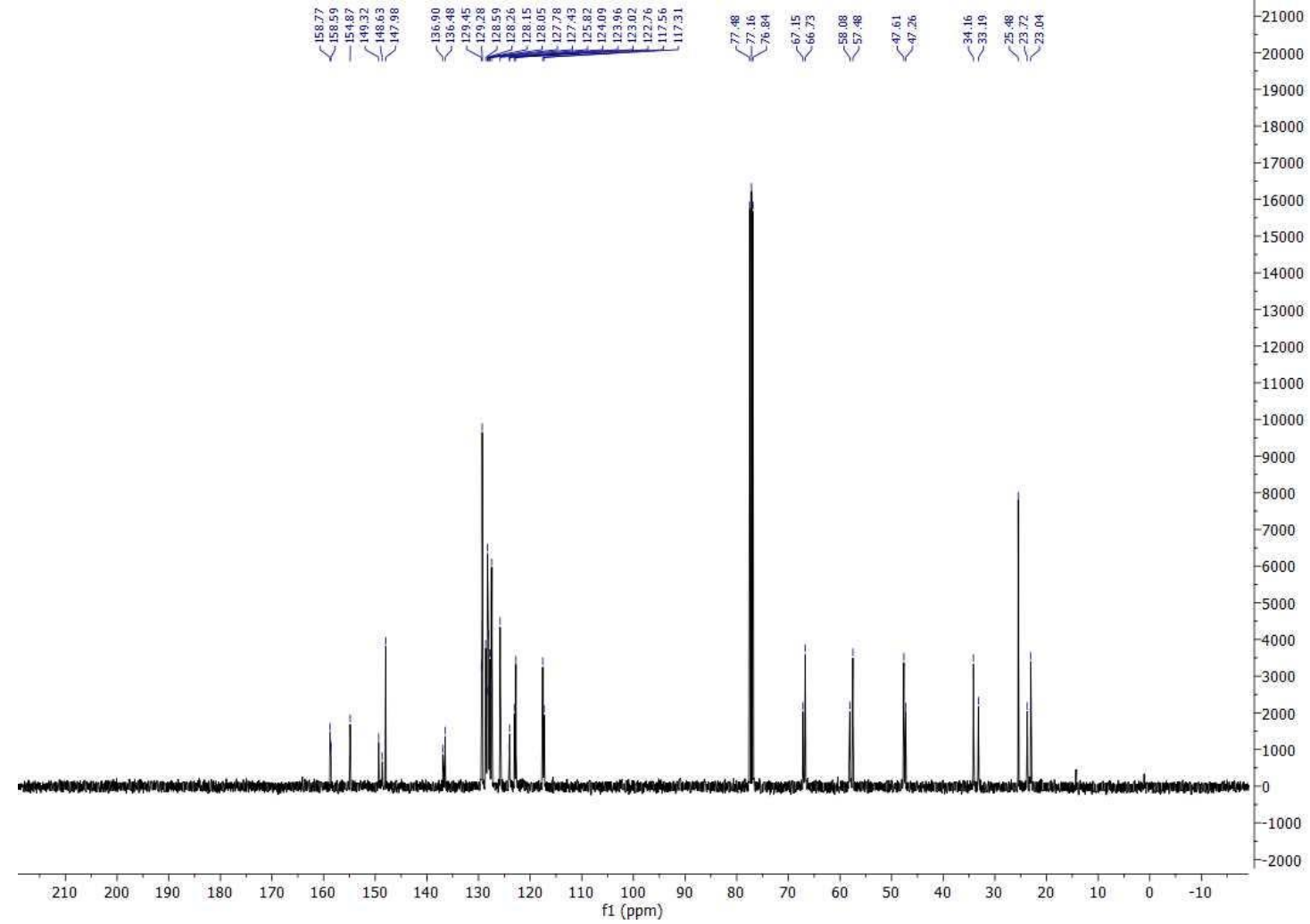


${ }^{1} \mathrm{H}$ NMR (400 MHz, $\left.\mathrm{CDCl}_{3}\right)$

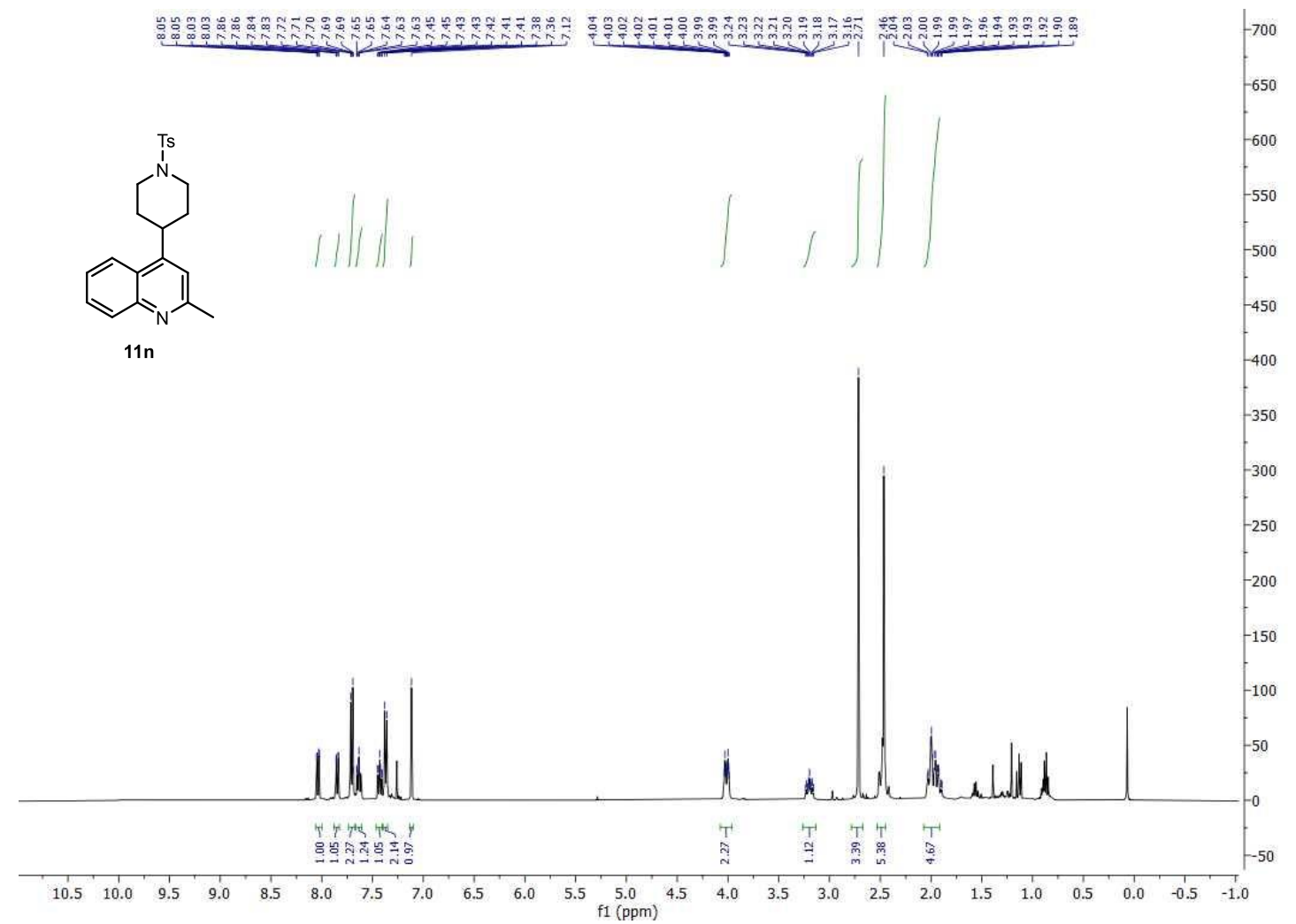

${ }^{13} \mathrm{C}$ NMR $\left(101 \mathrm{MHz}, \mathrm{CDCl}_{3}\right)$

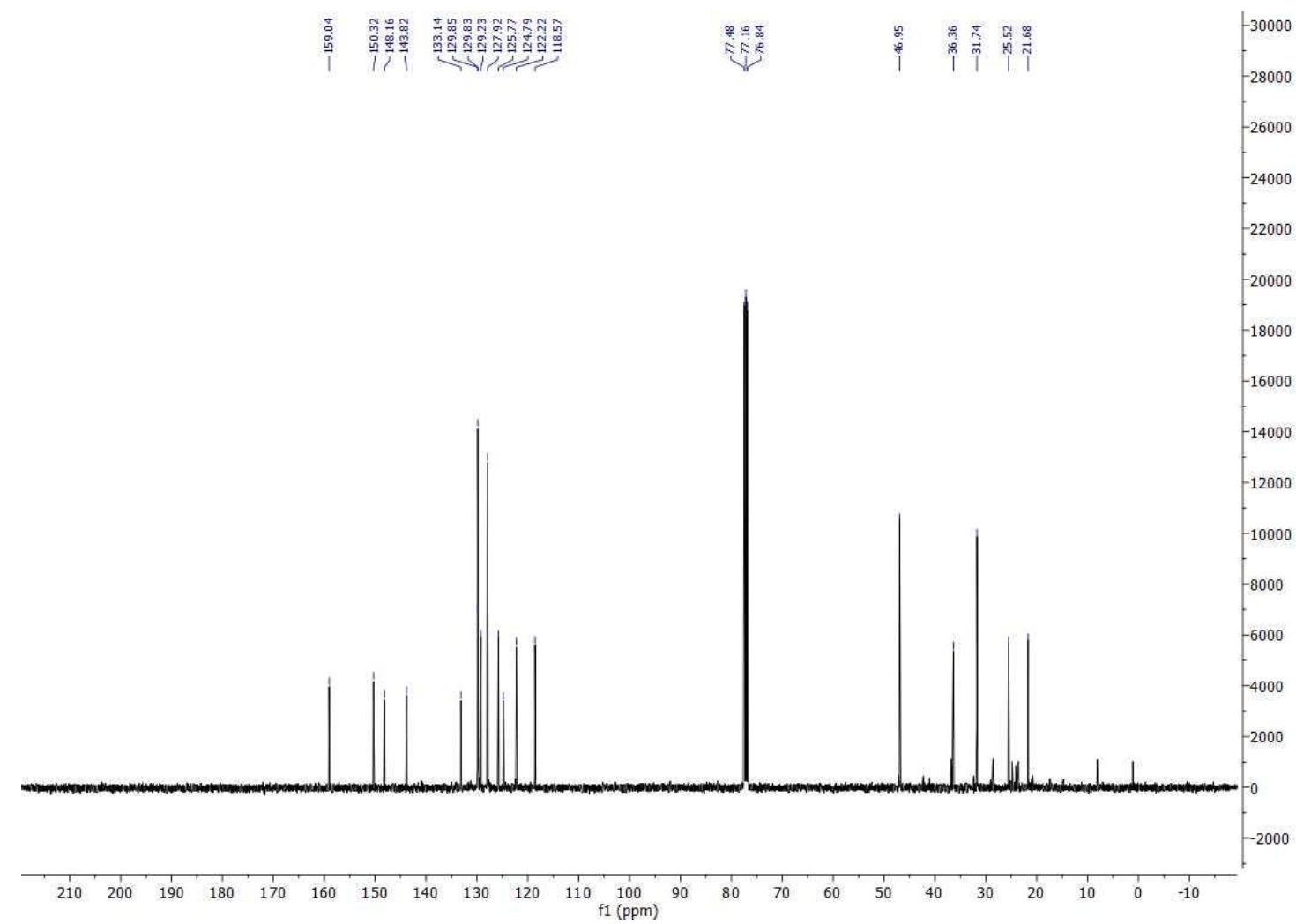


${ }^{1} \mathrm{H}$ NMR (400 MHz, $\mathrm{CDCl}_{3}$ )

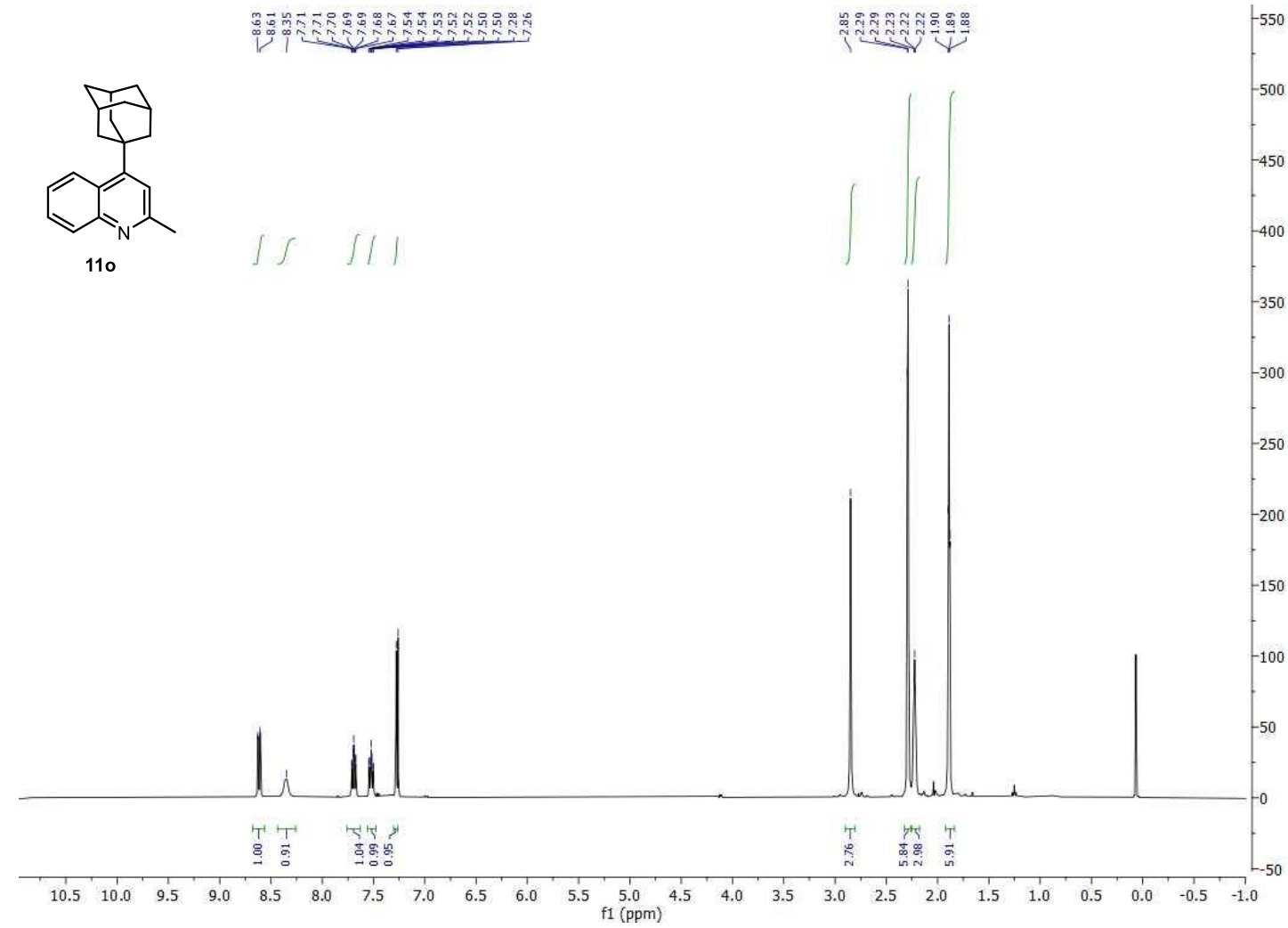

${ }^{13} \mathrm{C}$ NMR $\left(101 \mathrm{MHz}, \mathrm{CDCl}_{3}\right)$

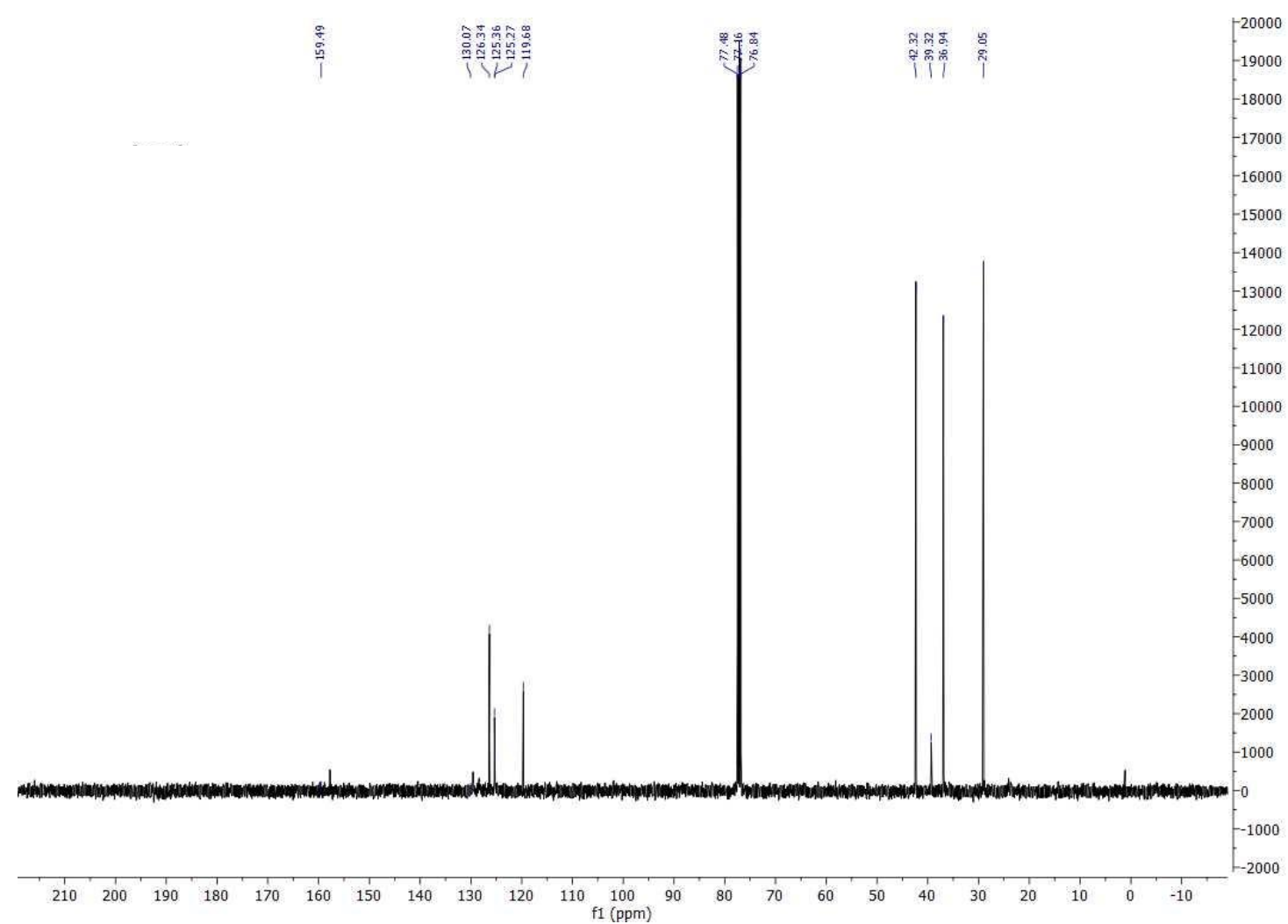


${ }^{1} \mathrm{H}$ NMR $\left(400 \mathrm{MHz}, \mathrm{CDCl}_{3}\right)$

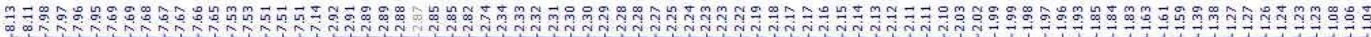

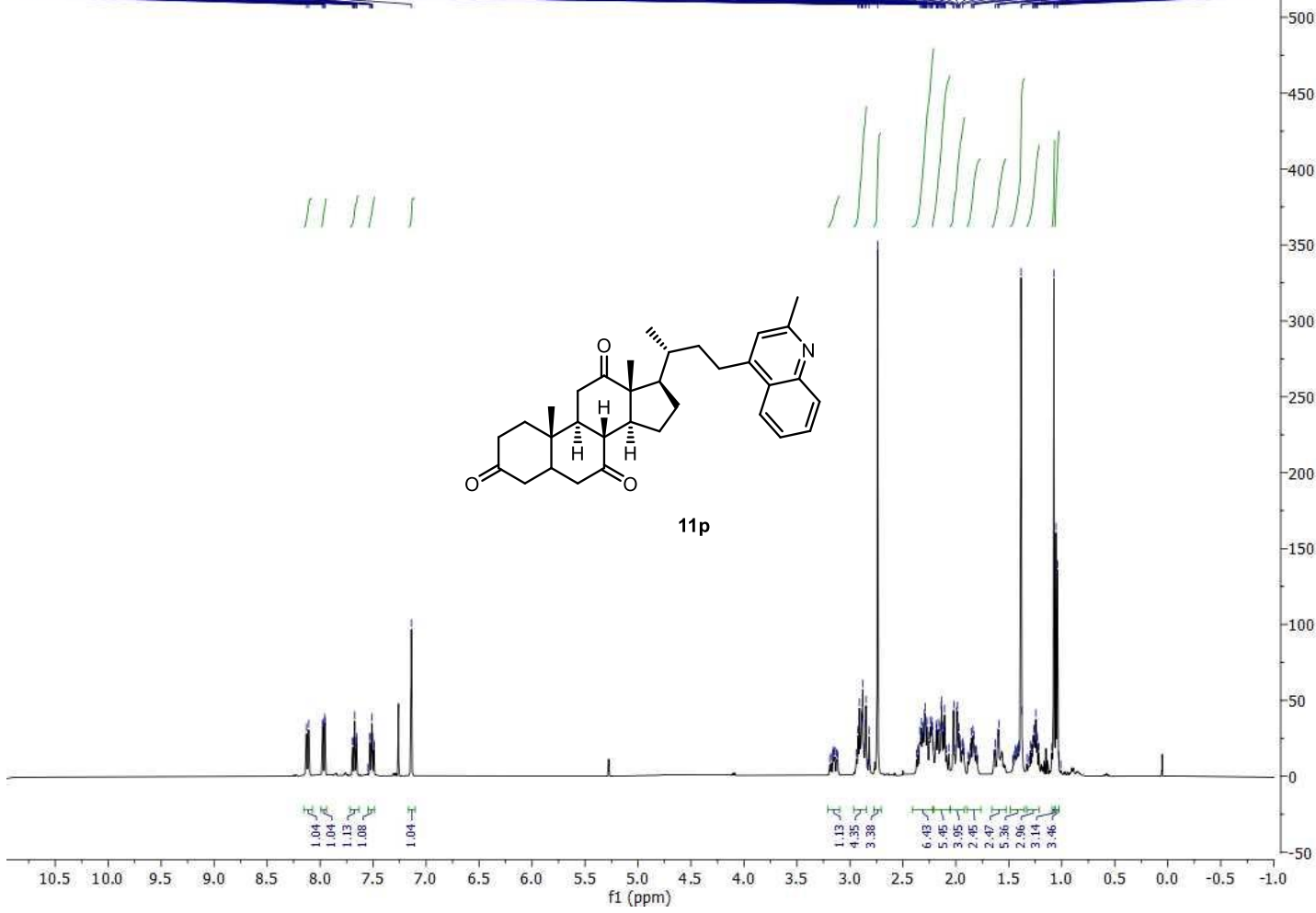

${ }^{13} \mathrm{C} \mathrm{NMR}\left(101 \mathrm{MHz}, \mathrm{CDCl}_{3}\right)$

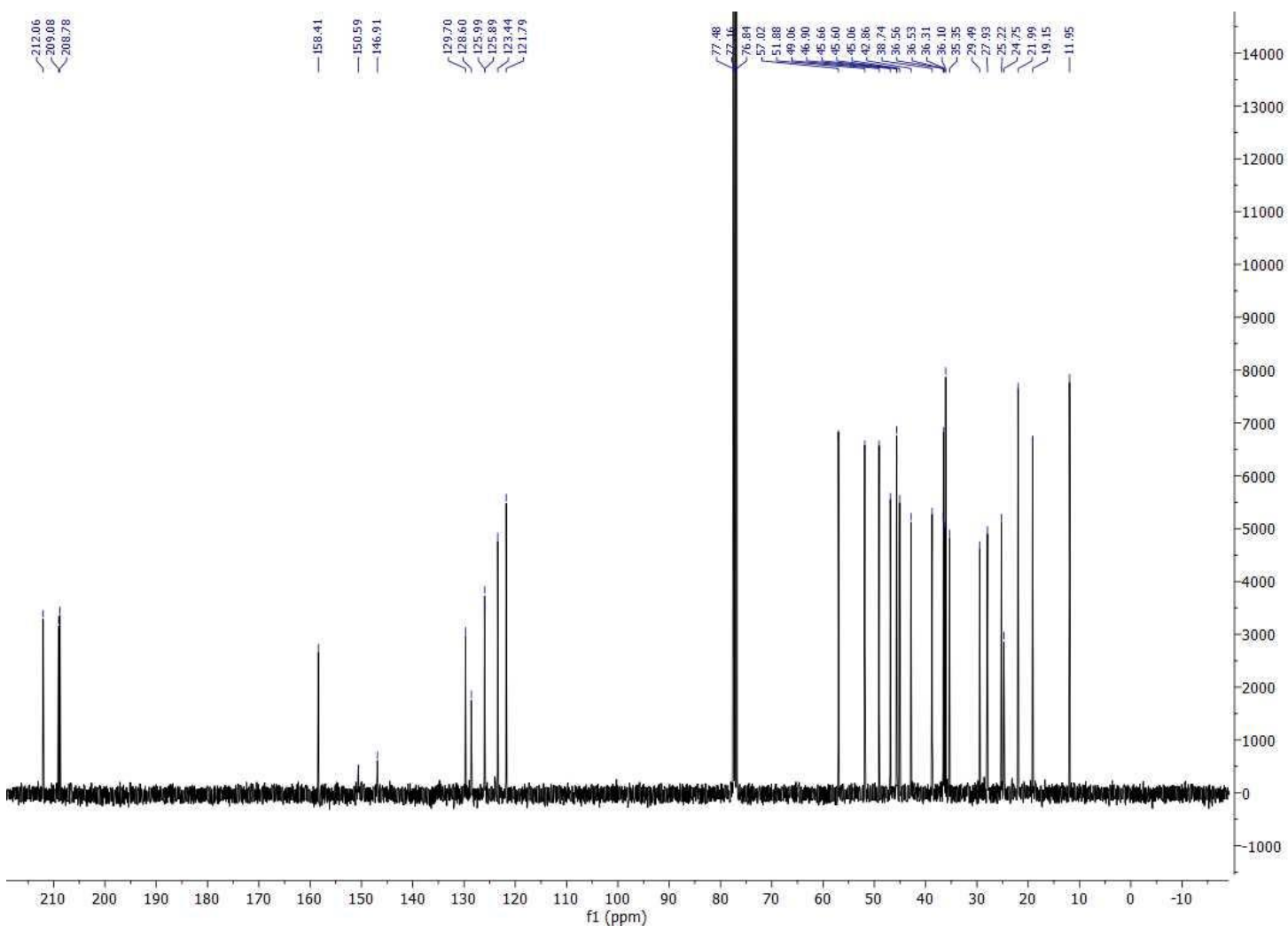


${ }^{1} \mathrm{H}$ NMR (400 MHz, $\mathrm{CDCl}_{3}$ )

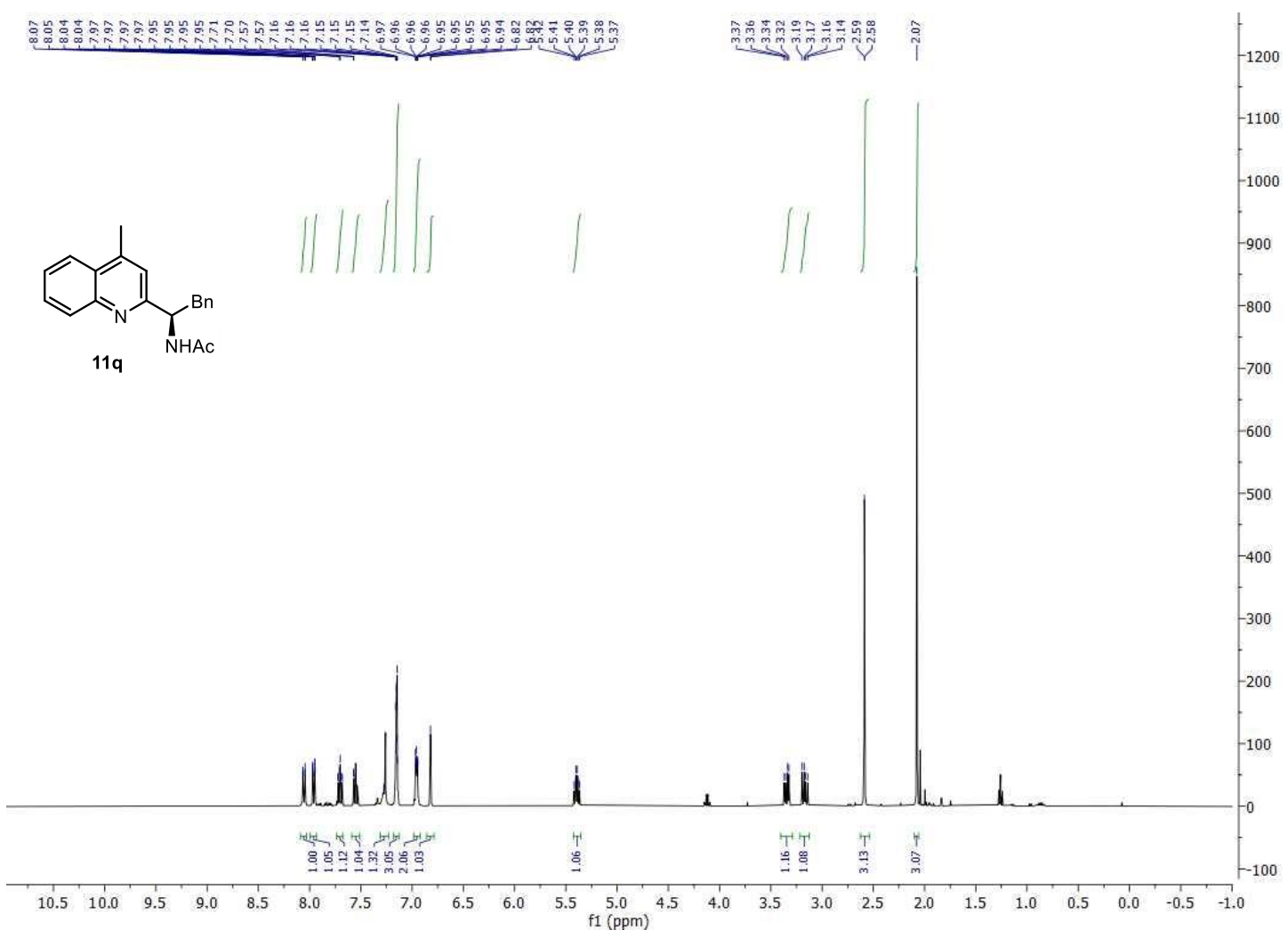

${ }^{13} \mathrm{C}$ NMR $\left(101 \mathrm{MHz}, \mathrm{CDCl}_{3}\right)$

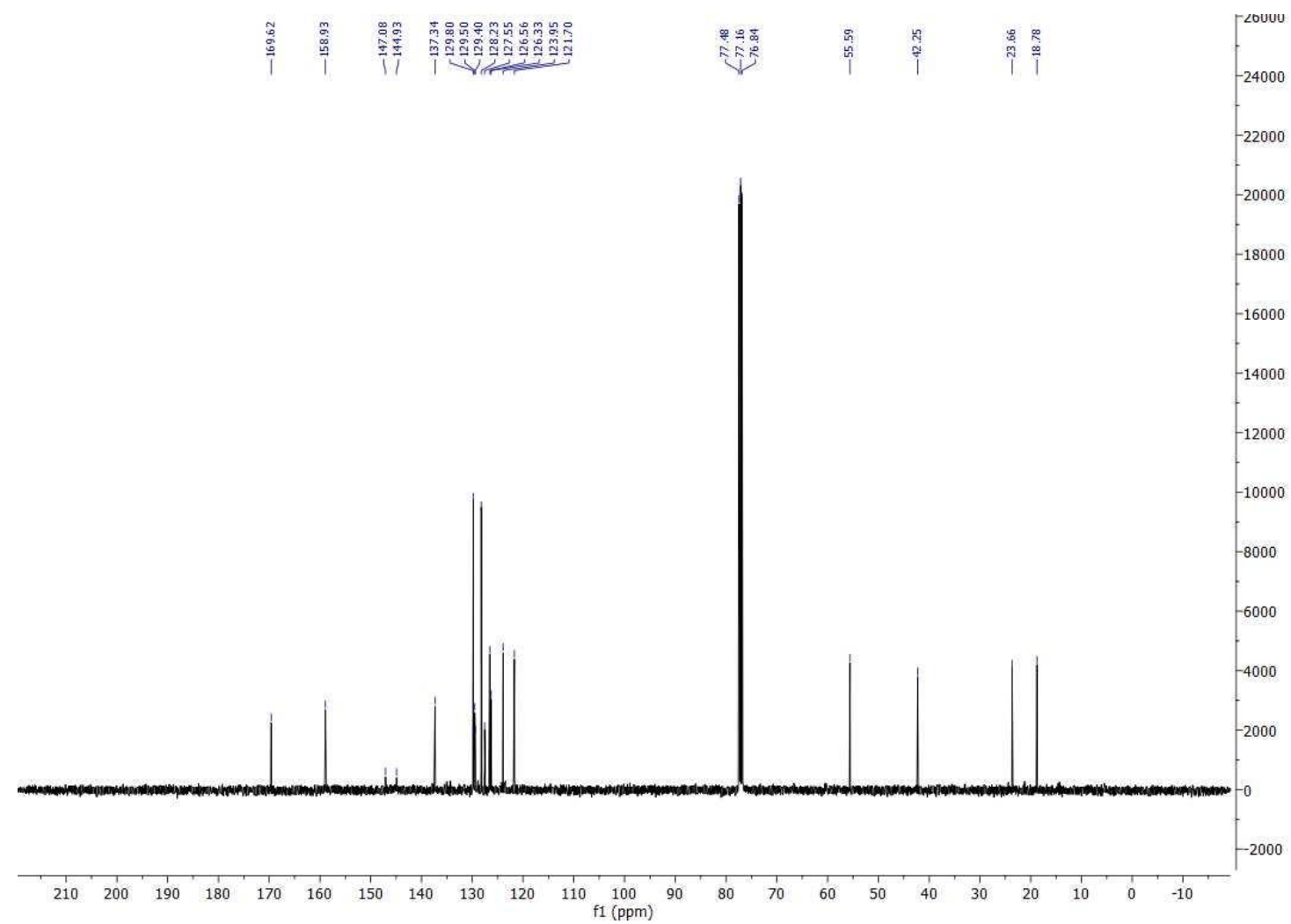


${ }^{1} \mathrm{H}$ NMR (400 MHz, $\mathrm{CDCl}_{3}$ )

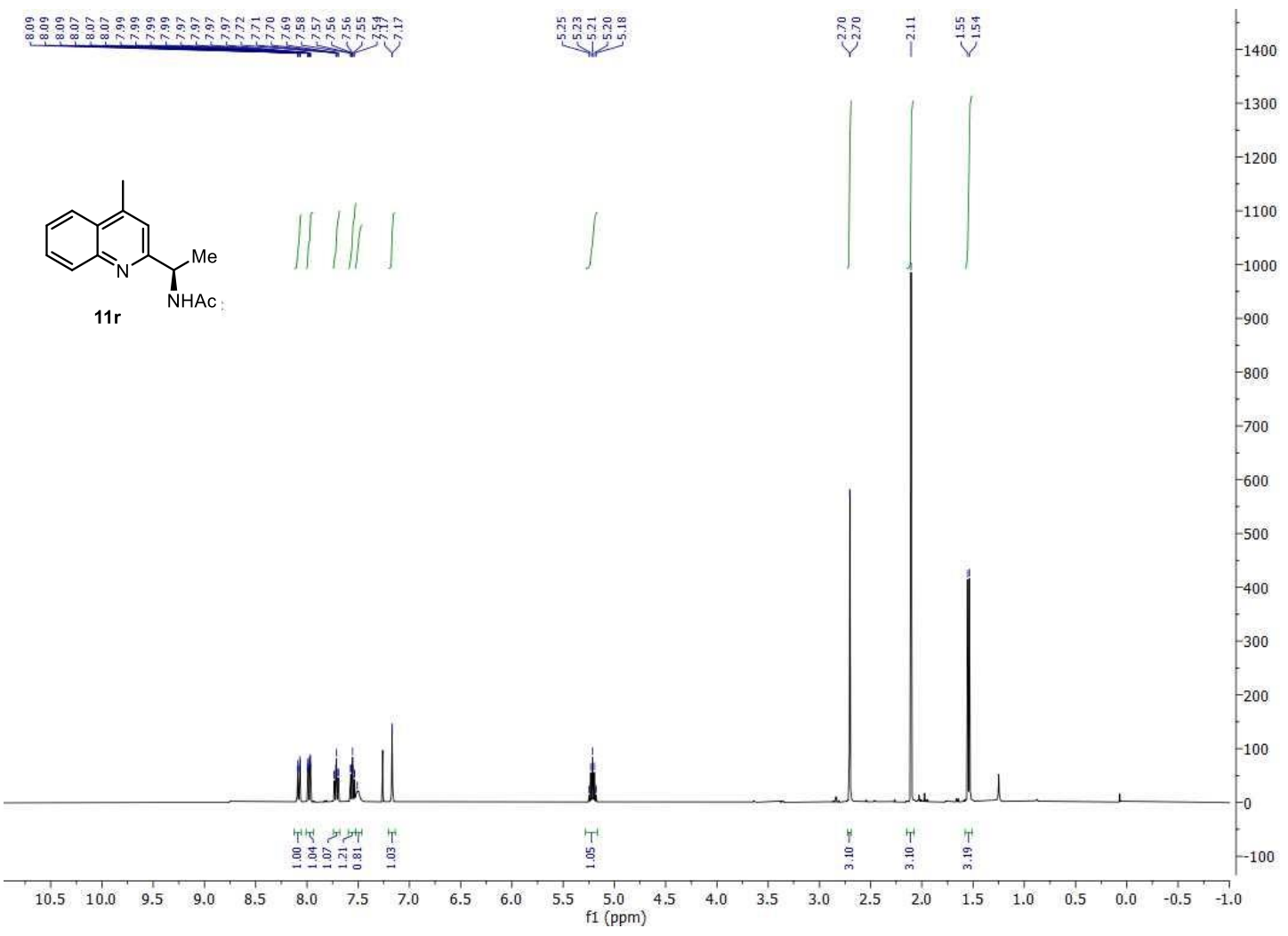

${ }^{13} \mathrm{C}$ NMR $\left(101 \mathrm{MHz}, \mathrm{CDCl}_{3}\right)$

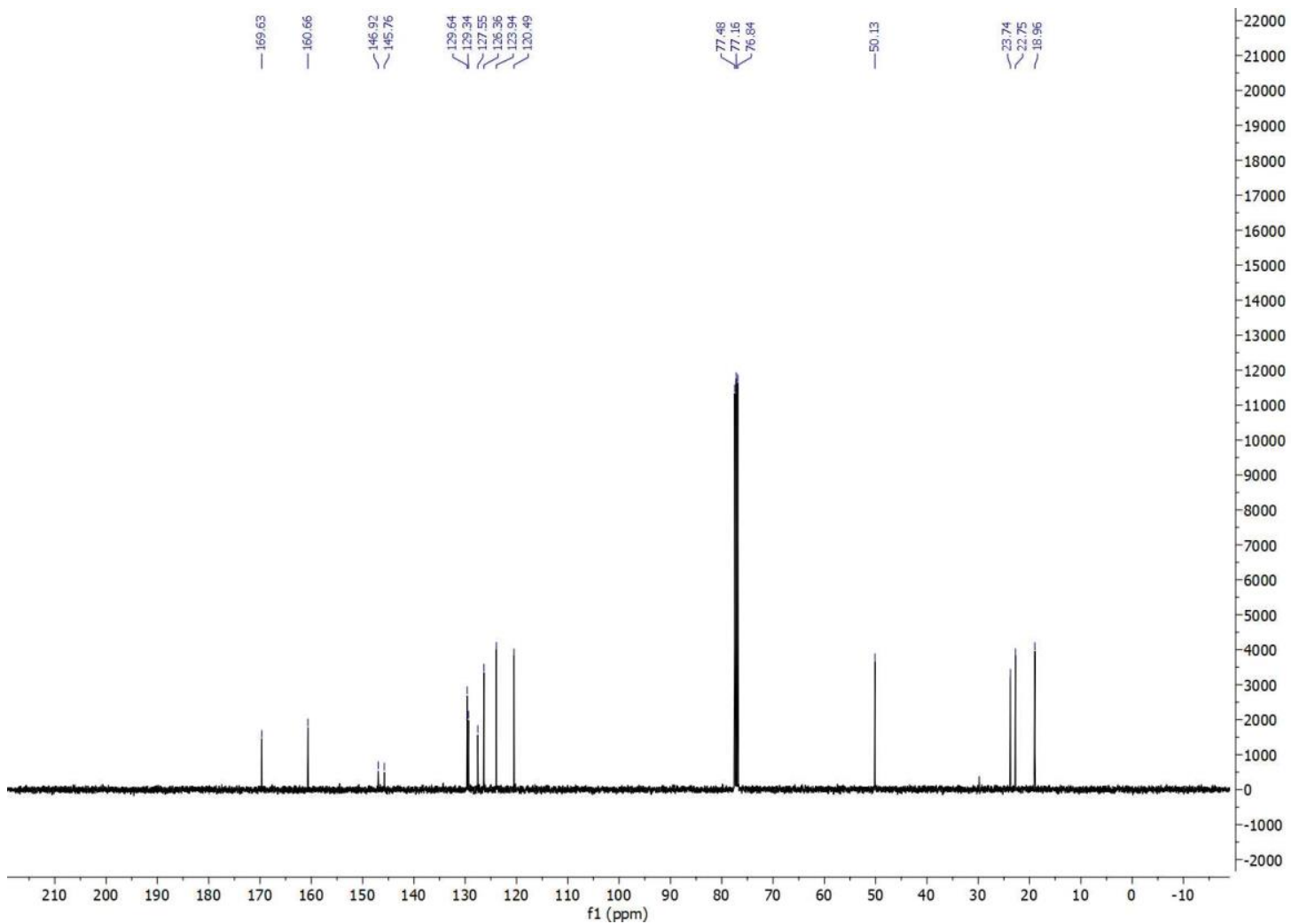


${ }^{1} \mathrm{H}$ NMR $\left(300 \mathrm{MHz}, \mathrm{CDCl}_{3}\right.$ )
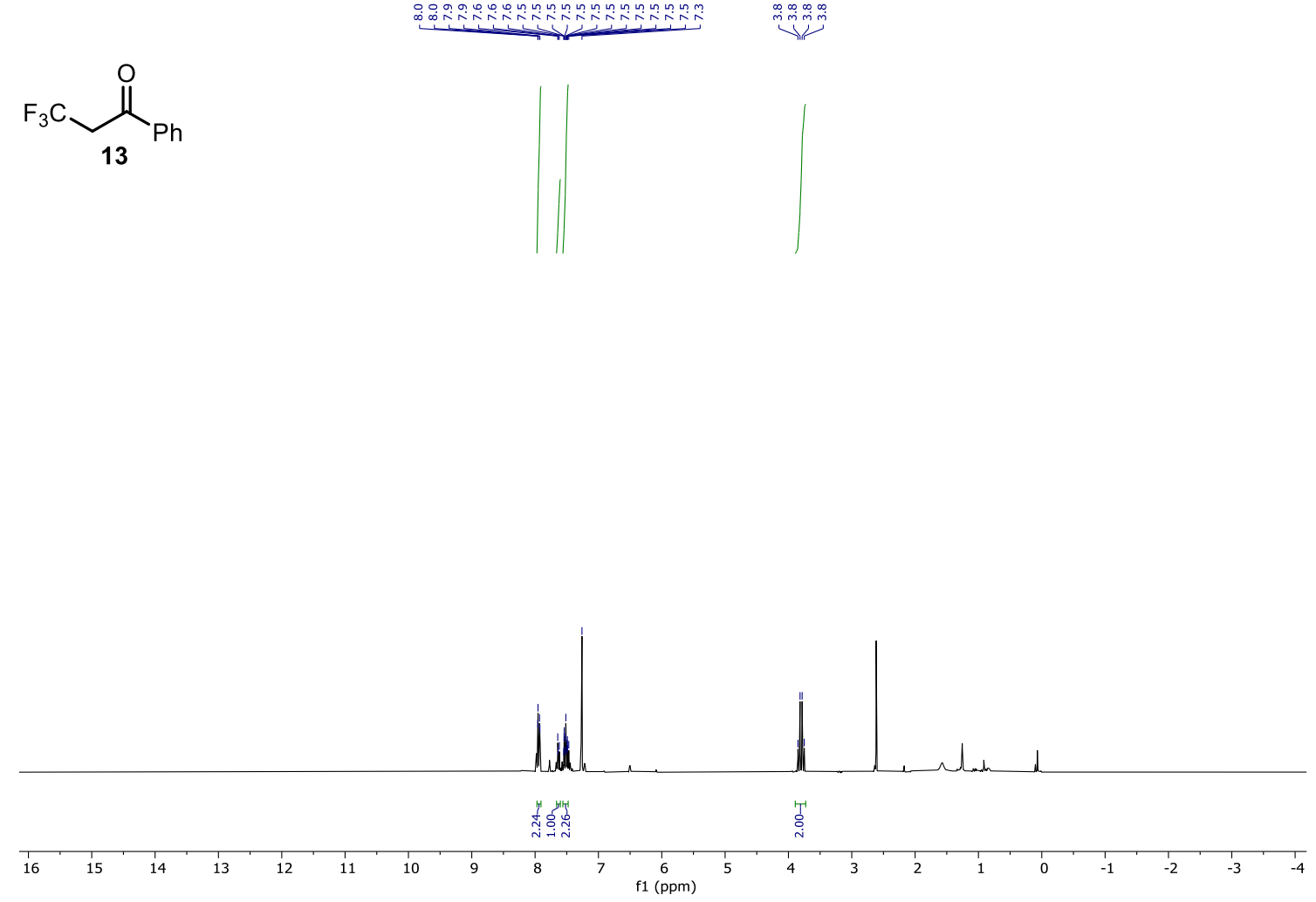

${ }^{13} \mathrm{C}$ NMR (126 MHz, CDCl3)

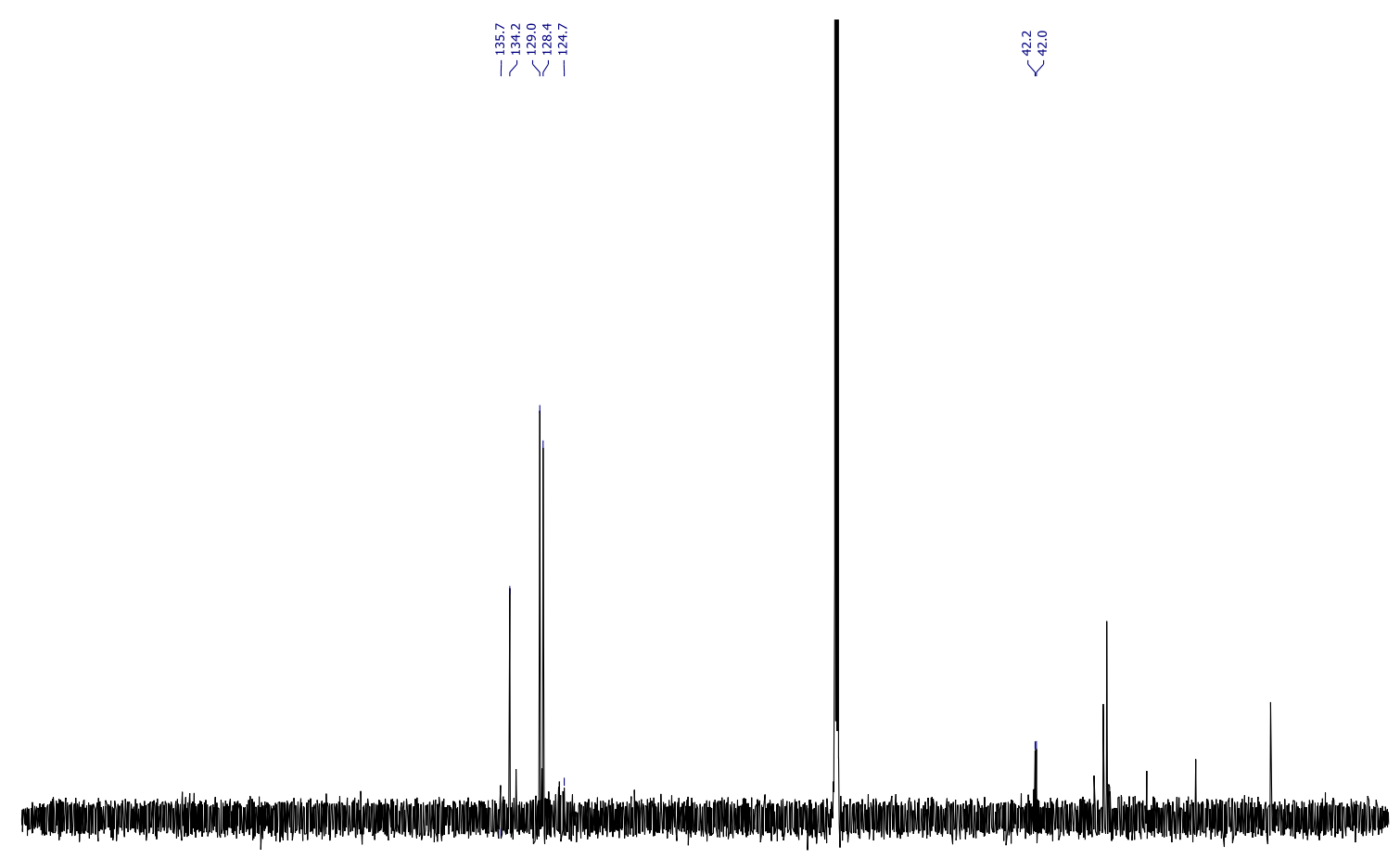

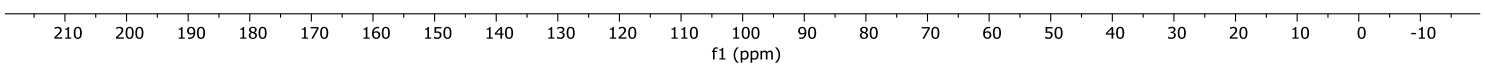


${ }^{19} \mathrm{~F}$ NMR $\left(376 \mathrm{MHz}, \mathrm{CDCl}_{3}\right)$

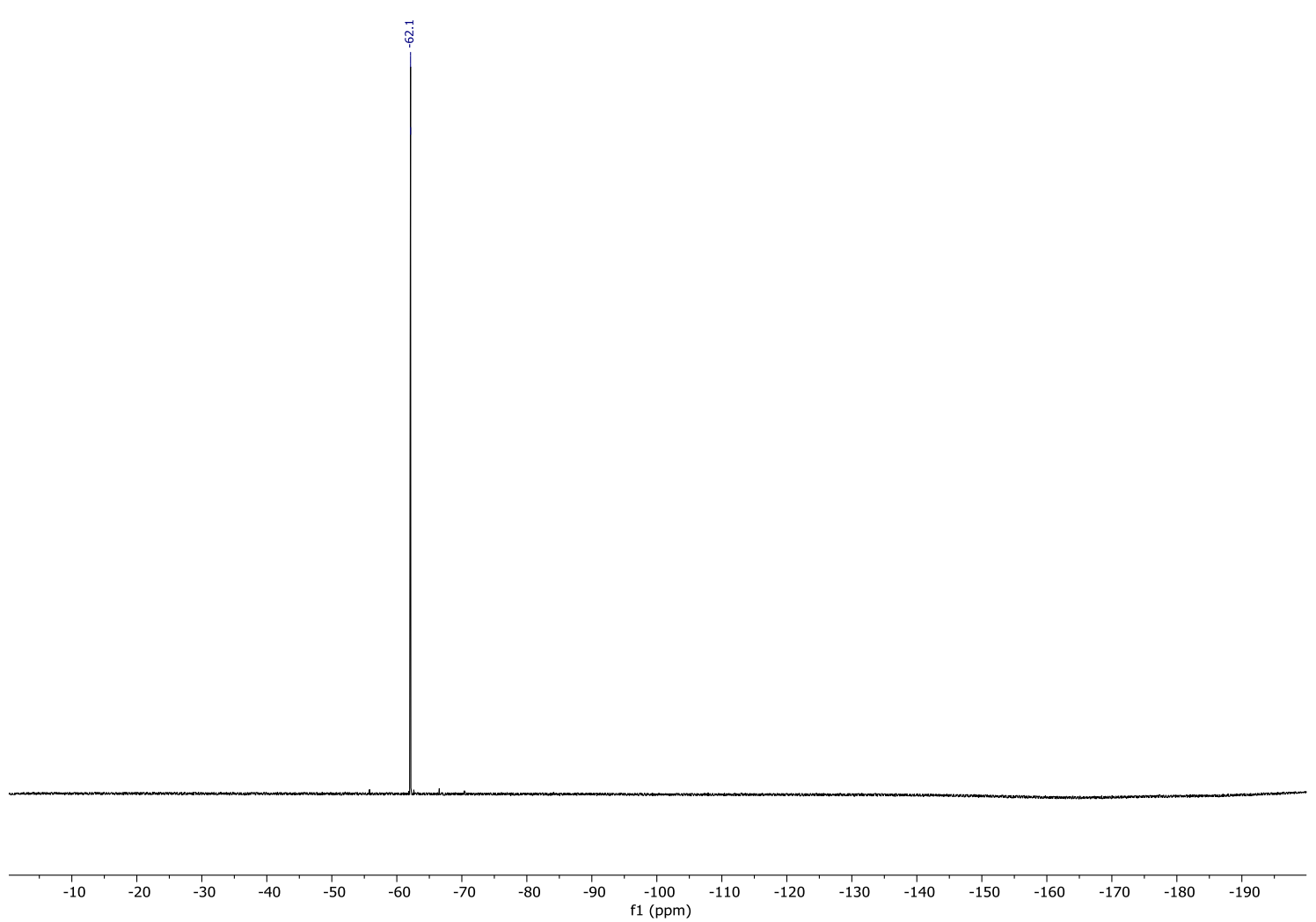


${ }^{1} \mathrm{H}$ NMR (500 MHz, $\mathrm{CDCl}_{3}$ )

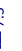

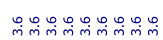

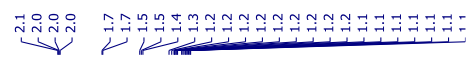<smiles>CC1(C)CCCC(C)(C)N1OC1CCCCC1</smiles>
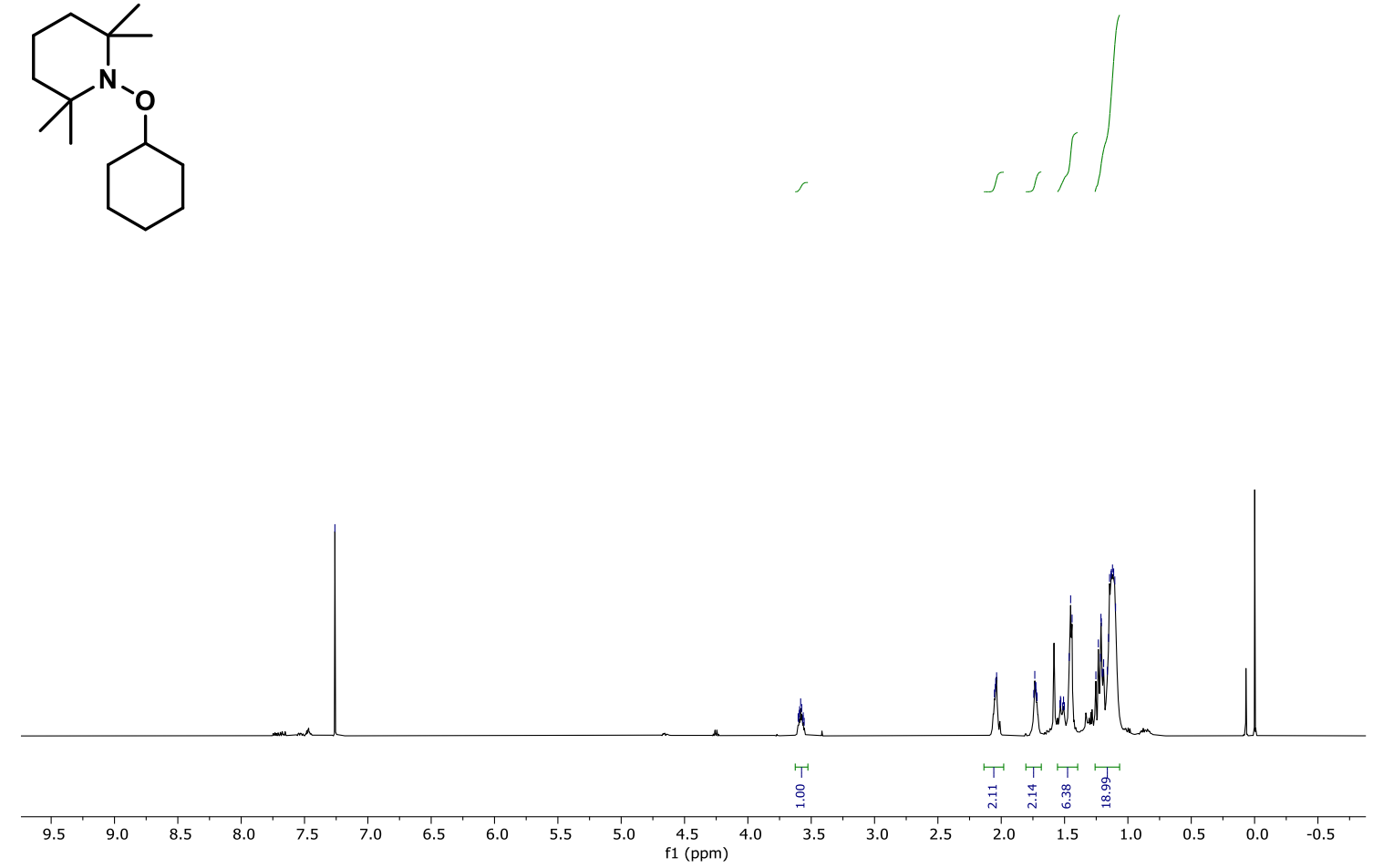

${ }^{13} \mathrm{C} \mathrm{NMR}\left(126 \mathrm{MHz}, \mathrm{CDCl}_{3}\right)$

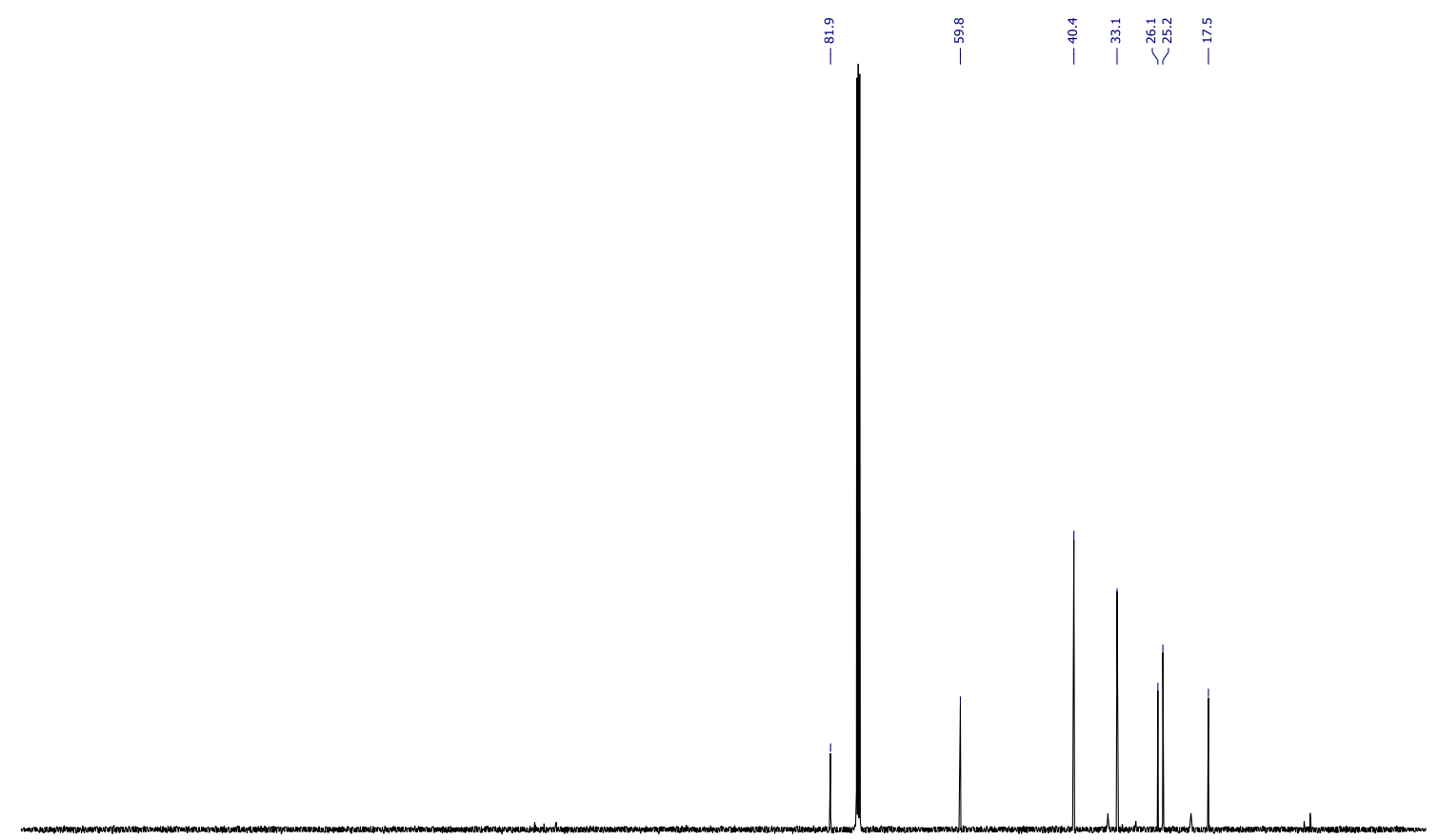


Conditions: HPLC analysis on a Daicel Chiralpak IC-3 column: 30:70 $\mathrm{PrOH}$ :nhexane $1.0 \mathrm{~mL} / \mathrm{min}$, $20 \stackrel{\circ}{ } \mathrm{C} ; \lambda=254 \mathrm{~nm}$.
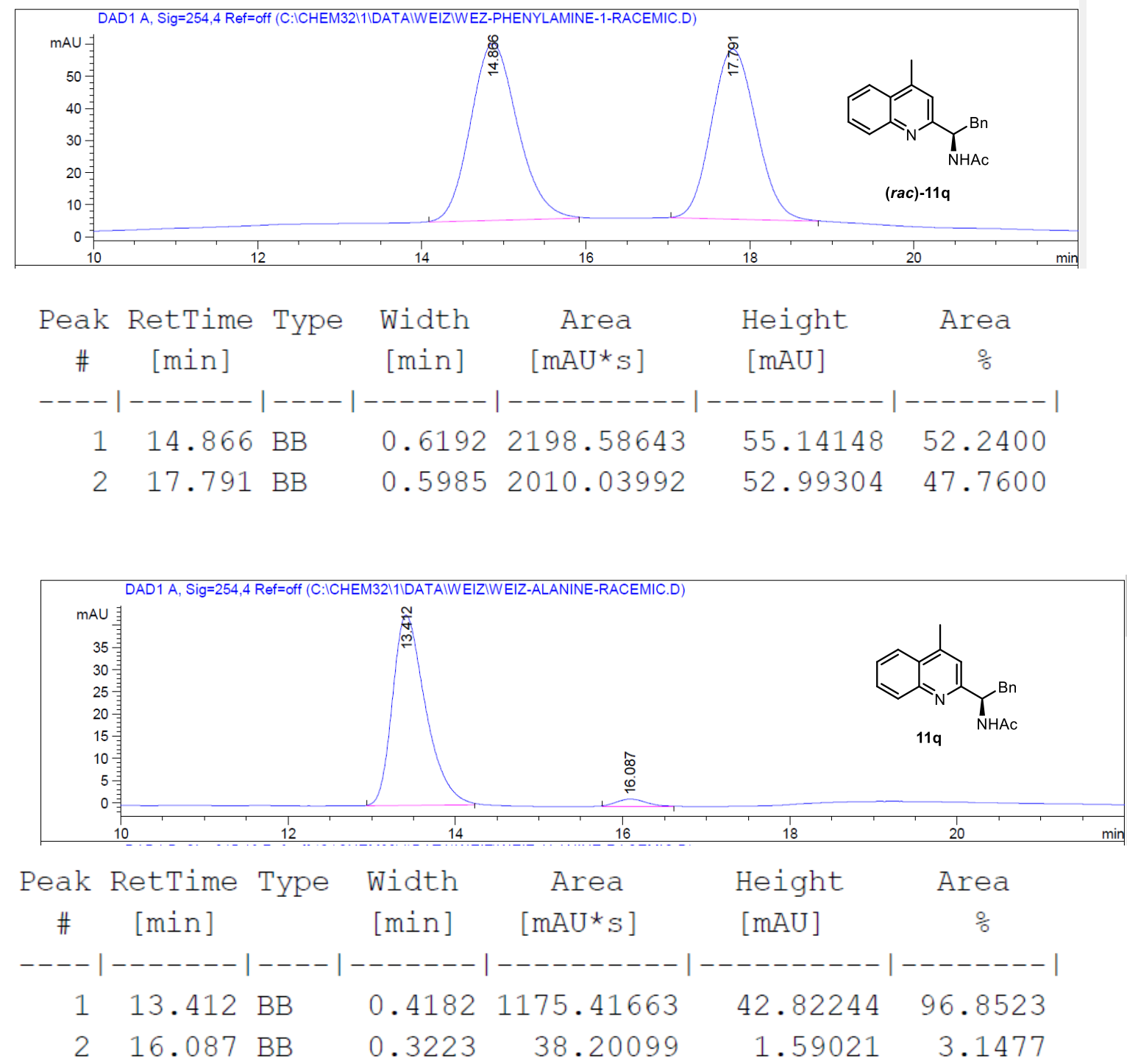
Conditions: HPLC analysis on a Daicel Chiralpak IC-3 column: $30: 70{ }^{\mathrm{i}} \mathrm{PrOH}$ :hexane $1.0 \mathrm{~mL} / \mathrm{min}$, 20 o $C ; \lambda=254 \mathrm{~nm}$.

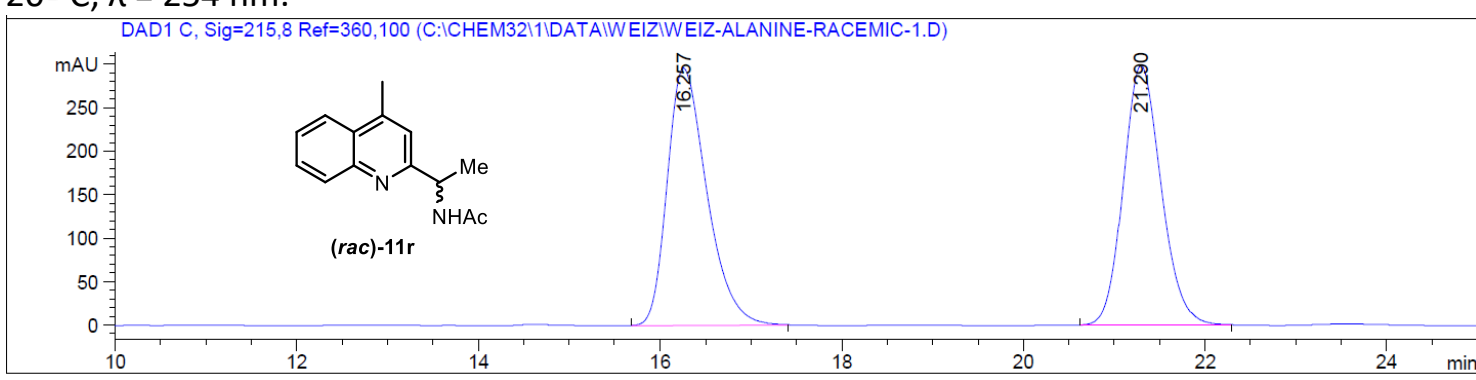

\begin{tabular}{|c|c|c|c|c|c|c|}
\hline $\begin{array}{c}\text { eak } \\
\#\end{array}$ & $\begin{array}{c}\text { RetTime } \\
\text { [min] }\end{array}$ & Type & $\begin{array}{l}\text { Width } \\
\text { [min] }\end{array}$ & $\begin{array}{c}\text { Area } \\
{\left[\mathrm{mAU}^{*} \mathrm{~s}\right]}\end{array}$ & $\begin{array}{l}\text { Height } \\
\text { [mAU] }\end{array}$ & $\begin{array}{c}\text { Area } \\
\text { \% }\end{array}$ \\
\hline & & & & & & \\
\hline 2 & & $\mathrm{BB}$ & & 8649.93359 & 9034 & \\
\hline
\end{tabular}

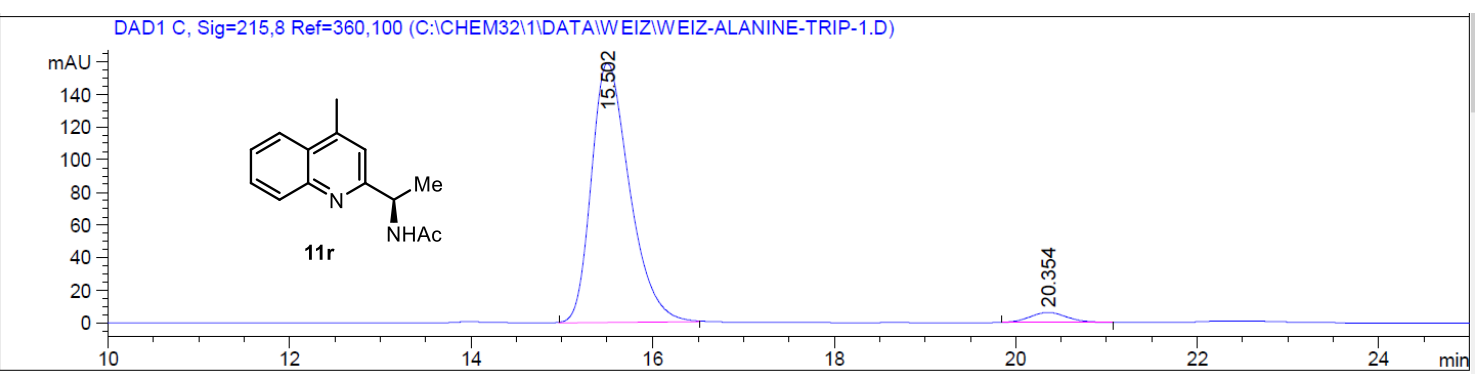

\begin{tabular}{|c|c|c|c|c|c|c|}
\hline $\begin{array}{c}\text { Peak } \\
\#\end{array}$ & $\begin{array}{c}\text { RetTime } \\
\text { [min] }\end{array}$ & Type & $\begin{array}{c}\text { Width } \\
\text { [min] }\end{array}$ & $\begin{array}{c}\text { Area } \\
{\left[\mathrm{mAU}^{*} \mathrm{~s}\right]}\end{array}$ & $\begin{array}{l}\text { Height } \\
{[\mathrm{mAU}]}\end{array}$ & $\begin{array}{c}\text { Area } \\
\frac{\circ}{\circ}\end{array}$ \\
\hline & & & & $\mid----------$ & -------- & --- \\
\hline 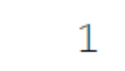 & & & & 16309 & 767 & 6 \\
\hline & & & & 176.97900 & 9149 & 6939 \\
\hline
\end{tabular}

